\title{
CHAOTIC TIME SERIES FORECASTING WITH RESIDUAL ANALYSIS USING SYNERGY OF ENSEMBLE NEURAL NETWORKS AND TAGUCHI'S DESIGN OF EXPERIMENTS
}

\author{
A dissertation \\ presented to Ryerson University \\ in partial fulfillment of the \\ requirements for the degree of \\ Doctor of Philosophy \\ in the program of \\ Mechanical Engineering
}




\section{AUTHOR'S DECLARATION}

I hereby declare that I am the sole author of this dissertation

I authorize Ryerson University to lend this dissertation to other institutions or individuals for the purpose of scholarly research.

I further authorize Ryerson University to reproduce this dissertation by photocopying or by other means, in total or in part, at the request of other institutions or individuals for the purpose of scholarly research. 


\section{BORROWER'S PAGE}

Ryerson University requires the signatures of all persons using or photocopying this dissertation. Please sign below and give the address and date. 


\begin{abstract}
Chaotic Time Series Forecasting with Residual Analysis Using Synergy of Ensemble Neural Networks and Taguchi's Design of Experiments

Doctor of Philosophy, 2010, Muhammad Ardalani-Farsa, Mechanical Engineering School of Graduate Studies, Ryerson University

This dissertation aims to develop an effective and practical method to forecast chaotic time series. Chaotic behaviour has been observed in the areas of marketing, stock markets, supply chain management, foreign exchange rates, weather forecasting and many others. An effective forecasting method can reduce the potential risks and uncertainty and facilitate planning and decision making in chaotic systems.

In this study, residual analysis using a combination of the embedding theorem and ensemble artificial neural networks is adopted to forecast chaotic time series. Based on the embedding theorem, the embedding parameters are determined and the time series is reconstructed into proper phase space points. The embedded phase space points are fed into the first neural network and trained. The weights and biases are kept to predict the future values of phase space points and accordingly to obtain future values of chaotic time series. The residual of the predicted time series is further analyzed; and, if a chaotic behaviour is observed, then the residuals are processed as a new chaotic time series and predicted. This iterative residual analysis can be repeated several times depending on the desired accuracy level and computational efficiency. Finally, the last neural network is trained using neural networks' result values of the time series and the residuals as input and the original time series as output. The initial weights and biases of the neural networks are improved using genetic algorithms. Taguchi's design of experiments is adopted to identify appropriate factor-level combinations to improve the result of the proposed forecasting method. A systematic approach is proposed to improve the combination of ensemble artificial neural networks and their parameters. The proposed methodology is applied to a number of benchmark and some real life chaotic time series. In addition, the proposed forecasting method has been applied to financial sector time series, namely, stock markets and foreign exchange rates. The experimental results confirm that the proposed method can predict the chaotic time series more effectively in terms of error indices when compared with other existing forecasting methods in the literature.
\end{abstract}




\section{ACKNOWLEDGEMENTS}

I am very thankful to my supervisor, Dr. Saeed Zolfaghari, whose guidance, support and encouragement from the initial to the final level allowed me to develop this dissertation. I owe my deepest gratitude to my parents who encouraged me to start my $\mathrm{PhD}$ and unconditionally supported me. It is an honour for me to thank the Director of Mechanical Engineering Graduate Program, Dr. Greg Kawall, for his abundant support. I am grateful to Dr. Kamran Behdinan for helping me to smoothly begin my PhD program. Further, I wish to thank the external examiner of my committee, Dr. Uday Venkatadri, committee chair, Dr. Ana Pejovic-Milic, and other members of the committee, Dr. Alireza Sadeghian, Dr. Liping Fang and Dr. Mohamad Jaber for their constructive comments and suggestions. I would like to offer my gratitude to all of those who have inspired and supported me in any respect during the completion of the dissertation. Lastly, I would like to thank Dr. Robert Roseberry for reviewing and proofreading my dissertation. 


\section{TABLE OF CONTENTS}

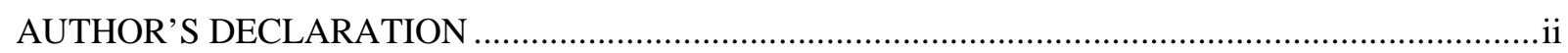

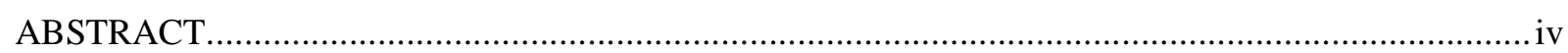

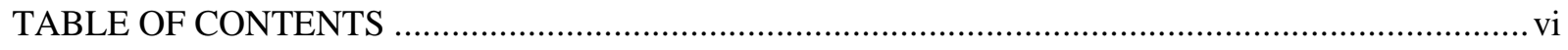

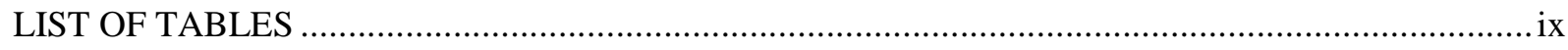

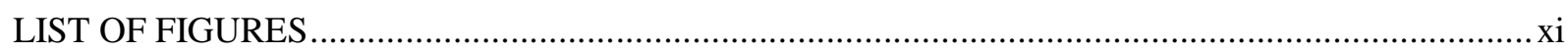

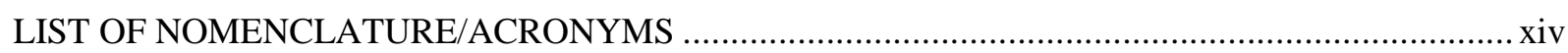

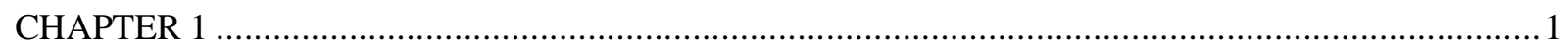

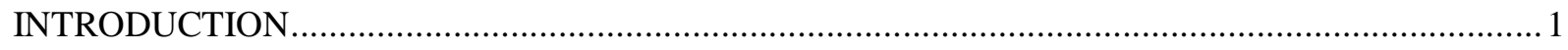

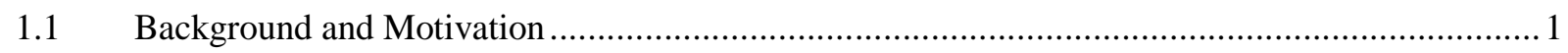

1.2 Overview of Chaos Theory and Chaotic Time Series Forecasting ...........................................2

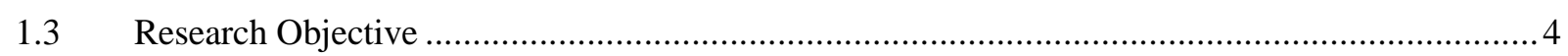

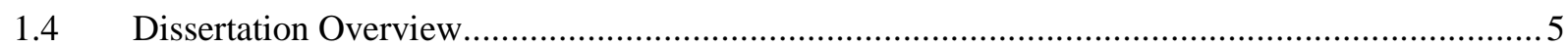

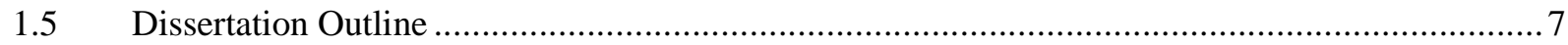

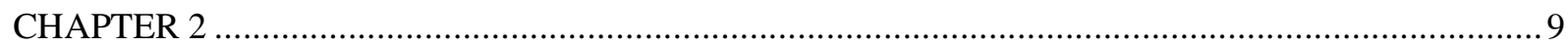

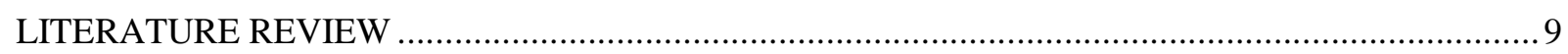

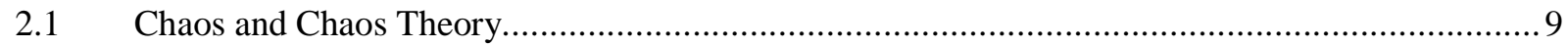

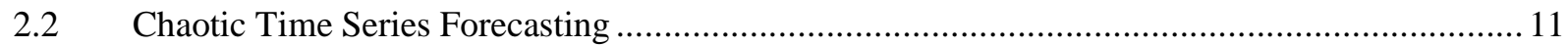

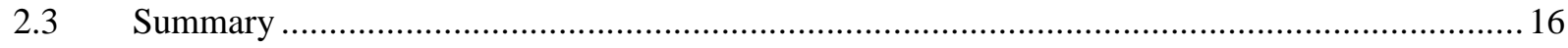

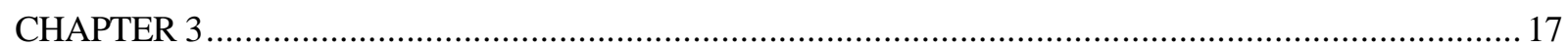

AN OVERVIEW OF THE EMBEDDING THEOREM AND ARTIFICIAL INTELLIGENCE .................. 17

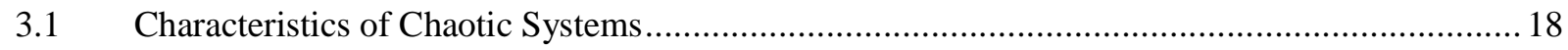

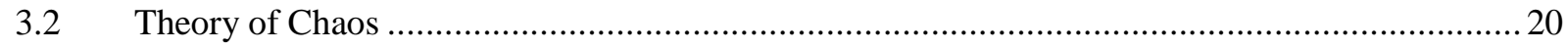

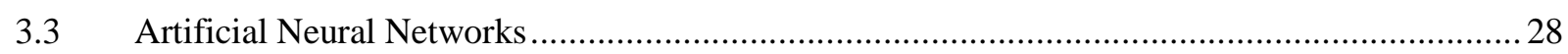

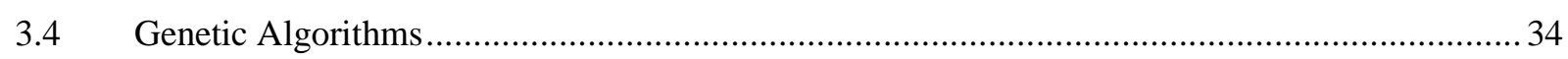

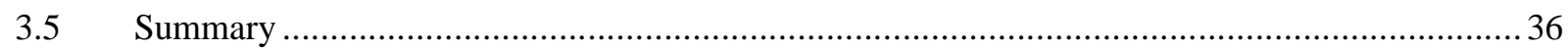


COMBINATION OF CHAOS THEORY AND ARTIFICIAL INTELLIGENCE IN CHAOTIC TIME

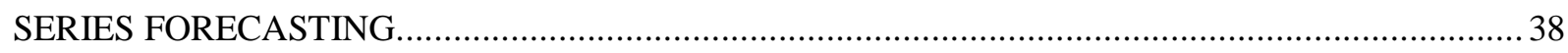

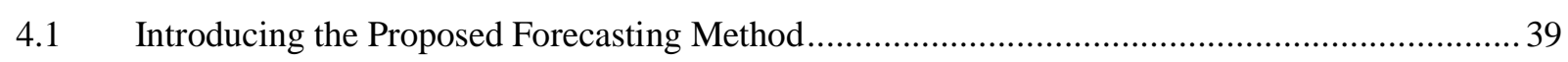

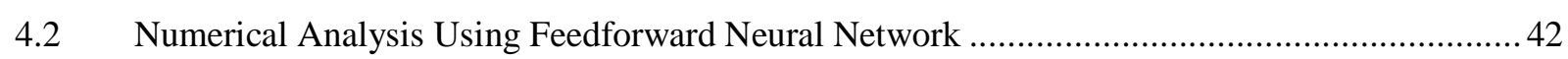

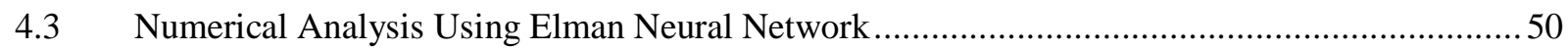

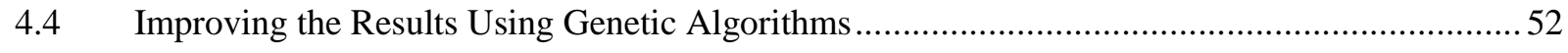

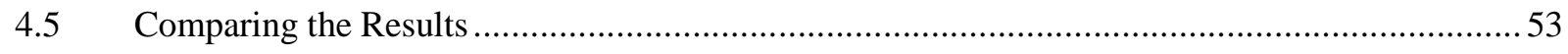

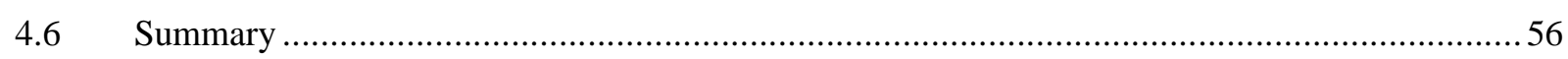

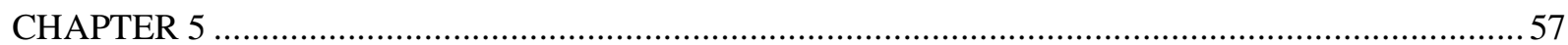

CHAOTIC TIME SERIES FORECASTING WITH RESIDUAL ANALYSIS USING ENSEMBLE

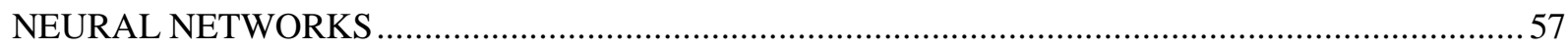

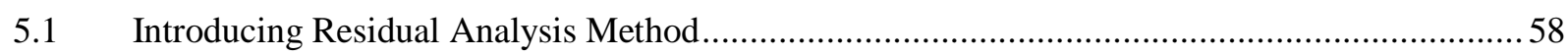

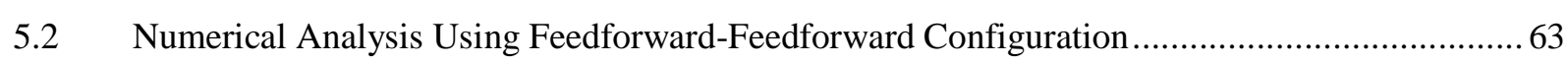

5.3 Numerical Analysis Using Elman-NARX Configuration .......................................................... 73

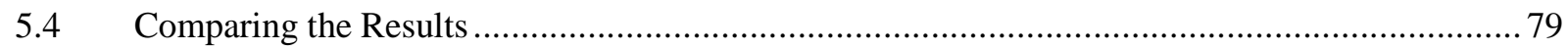

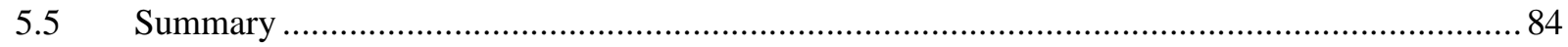

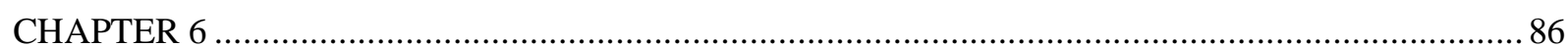

TAGUCHI'S DOE IN COMBINATION SELECTION FOR CHAOTIC TIME SERIES FORECASTING

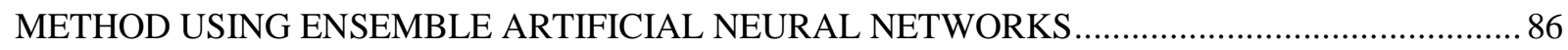

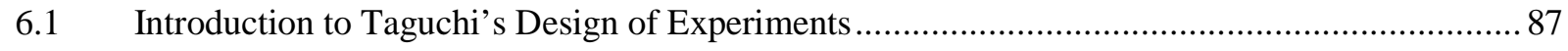

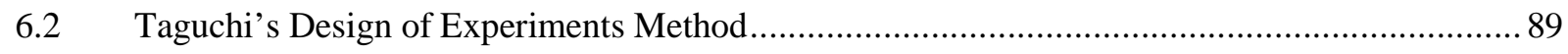

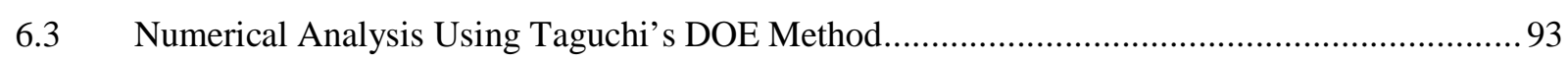

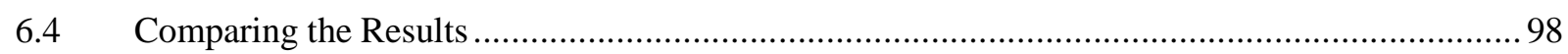

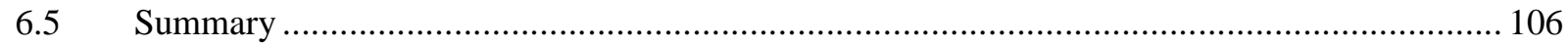

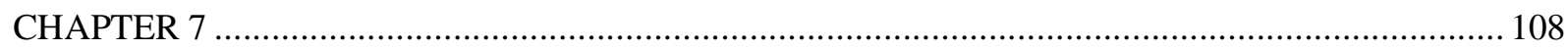

APPLICATIONS OF CHAOTIC TIME SERIES FORECASTING .................................................. 108

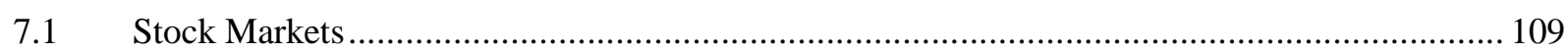

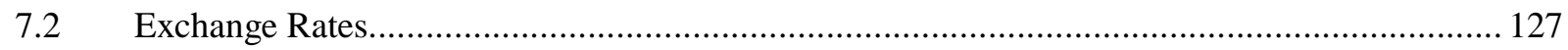

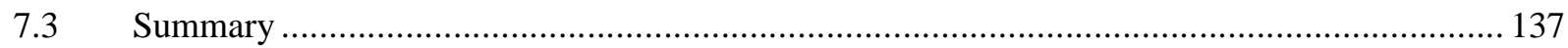


CHAPTER 8.

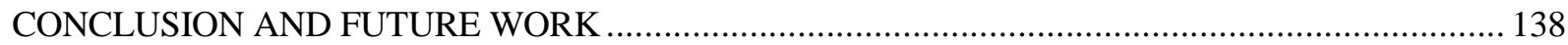

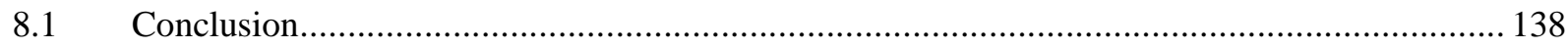

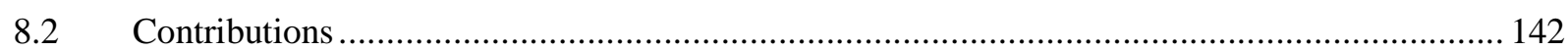

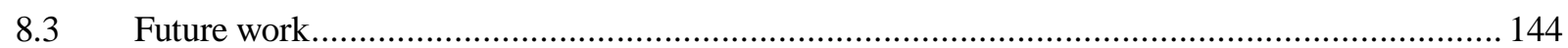

Appendix A - A Numerical Example for Chaos Theory and Embedding Theorem .............................. 147

Appendix B - A Numerical Example to Detect the Existence of Chaos ................................................ 150

Appendix C - A Numerical Example to Determine Embedding Parameters .......................................... 153

Appendix D - Regression Analysis for Artificial Neural Networks ...................................................... 156

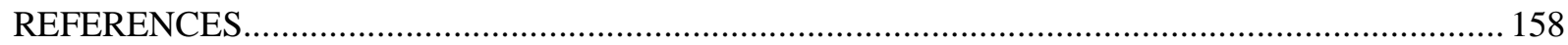




\section{LIST OF TABLES}

Table 4.1 Mackey-Glass time series - The MSE and NMSE for the 500 test samples.

Table 4.2 Logistic time series - The MSE and NMSE for the 100 test data points.

Table 4.3 Henon time series - The MSE and NMSE for the 500 test data points.

Table 4.4 The prediction performance using Elman RNN.

Table 4.5 The prediction performance improved by genetic algorithms.

Table 4.6 Mackey-Glass time series - comparing the prediction performance.

Table 4.7 Logistic time series - comparing the prediction performance.

Table 4.8 Henon time series - comparing the prediction performance.

Table 5.1 Mackey-Glass time series - the error indices for FFNN-FNNN configuration.

Table 5.2 Lorenz time series - the error indices for FFNN-FNNN configuration.

Table 5.3 Mackey-Glass time series - the error indices for Elman-NARX configuration.

Table 5.4 Lorenz time series - the error indices for Elman-NARX configuration.

Table 5.5 Sunspot time series - the error indices for Elman-NARX configuration.

Table 5.6 Comparison between the prediction errors reported in the literature and the proposed method (500 Mackey-Glass time series test samples).

Table 5.7 Comparison between the prediction errors reported in the literature and the proposed method (1000 Lorenz time series test samples).

Table 5.8 Comparison between the prediction errors reported in the literature and the proposed method (1000 sunspot time series test samples).

Table 6.1 The control factors and their associated levels.

Table 6.2 Taguchi's DOE setting; orthogonal array L16 $\left(2^{8}\right)$.

Table 6.3 Taguchi's DOE setting; orthogonal array L16 $\left(2^{8}\right)$.

Table 6.4 The result of ANOVA analysis. 
Table 6.5 The response table for Lorenz time series, Mackey-Glass time series, sunspot time series and farinfrared NH3 laser time series.

Table 6.6 Comparison between the prediction errors reported in the literature and the proposed method (1000 Lorenz time series validation data).

Table 6.7 Comparison between the prediction errors reported in the literature and the proposed method (500 Mackey-Glass time series validation data).

Table 6.8 Comparison between the prediction errors reported in the literature and the proposed method (1000 sunspot time series validation data).

Table 6.9 Comparison between the prediction errors reported in the literature and the proposed method (100 far-infrared NH3 laser time series validation data).

Table 7.1 Comparison between the prediction errors reported in the literature and the proposed method (94 British Airlines stock test data).

Table 7.2 Comparison between the prediction errors reported in the literature and the proposed method (82 Southwest Airlines stock test data).

Table 7.3 Comparison between the prediction errors reported in the literature and the proposed method (70 Ryanair stock test data).

Table 7.4 Comparison between the prediction errors reported in the literature and the proposed method (500 Lucent stock test data).

Table 7.5 Comparison between the prediction errors reported in the literature and the proposed method (157 EUR/USD test data).

Table 7.6 Comparison between the prediction errors reported in the literature and the proposed method (500 USD/JPY test data).

Table D.1 The regression analysis between the proposed Elman and feedforward neural network response and the corresponding targets when applied to Mackey-Glass, Logistic and Henon time series. 


\section{LIST OF FIGURES}

Figure 1.1 A block diagram demonstrating the proposed methodology.

Figure 3.1 Single node in a feedforward neural network.

Figure 3.2 The architecture of feedforward neural network.

Figure 3.3 The architecture of Elman recurrent neural network.

Figure 3.4 The architecture of NARX neural network.

Figure 3.5 A string representing gene and chromosome in genetic algorithms.

Figure 3.6 Schematic illustration of genetic algorithms.

Figure 4.1 A block diagram demonstrating the proposed forecasting method.

Figure 4.2 Mackey-Glass chaotic time series a) in time domain, b) in phase space domain.

Figure 4.3 Comparison of Mackey-Glass time series and forecast values using FFNN.

Figure 4.4 Logistic chaotic time series a) in time domain, b) in phase space domain.

Figure 4.5 Comparison of Logistic time series data and forecast values using FFNN.

Figure 4.6 Henon chaotic time series a) in time domain, b) in phase space domain.

Figure 4.7 Comparison of Henon time series data and forecast values using FFNN.

Figure 4.8 Comparison of M-G time series data and forecast values using Elman ANN.

Figure 4.9 Comparison of Logistic time series data and forecast values using Elman RNN.

Figure 4.10 Comparison of Henon time series data and forecast values using Elman RNN.

Figure 5.1 A FFNN correlating the original and residual time series prediction to original time series.

Figure 5.2 A NARX network correlating the original and residuals forecasts to original time series.

Figure 5.3 A FFNN correlating the original time series prediction and the prediction of residuals in various levels to original time series.

Figure 5.4 Mackey-Glass Time Series - Comparing the original time series data and the predicted values.

Figure 5.5 Mackey-Glass time series -First residual values a) in the time domain b) in the phase space domain. Figure 5.6 Mackey-Glass time series - The comparison of the first residual values and the predicted values. 
Figure 5.7 Mackey-Glass time series - The second residual values, a)in time domain b) in phase space domain.

Figure 5.8 Mackey-Glass time series - The comparison of the second residual values and the predicted values.

Figure 5.9 The comparison between original Mackey-Glass time series and the final predicted values.

Figure 5.10 Lorenz time series, a) in time domain, b) in phase space domain.

Figure 5.11 Lorenz time series - The comparison of the original time series samples and the predicted values based on feedforward network.

Figure 5.12 Lorenz time series - The first residuals a) in time domain, b) in phase space domain.

Figure 5.13 Lorenz time series - the comparison of the first residual and predicted values.

Figure 5.14 The comparison between original Lorenz time series and the final predicted values.

Figure 5.15 Sunspot time series - The comparison of the original time series samples and the predicted values based on Elman network.

Figure 5.16 The comparison between original sunspot time series and the final predicted values.

Figure 6.1 The comparison between original Lorenz time series and the final predicted values.

Figure 6.2 Comparison between original Mackey-Glass time series and the final predicted values.

Figure 6.3 The comparison between original sunspot time series and the final predicted values.

Figure 6.4 The marked area in Figure 6.3.

Figure 6.5 Comparison between original far-infrared NH3 laser series and final forecast values.

Figure 7.1 The daily closing price of British Airlines stock for Jan. 1, 2003 to Jan. 10, 2005.

Figure 7.2 Largest Lyapunov exponent graph for British Airlines stock data.

Figure 7.3 British Airlines stock data in phase space domain.

Figure 7.4 British Airlines stock data - Comparing the original stock data and the predicted values.

Figure 7.5 British Airlines stock data - The comparison of the first residual values and the predicted values.

Figure 7.6 British Airlines stock data - The comparison of the second residual values and the predicted values.

Figure 7.7 The comparison between original British Airlines stock data and the final predicted values.

Figure 7.8 The daily closing price of Southwest Airlines stock December 18, 2002 to November 17, 2004. 
Figure 7.9 The comparison between original Southwest Airlines stock data and the final predicted values.

Figure 7.10 The daily closing price of Ryanair stock for the period May 06, 2003 to March 17, 2005.

Figure 7.11 The comparison between original Ryanair stock data and the final predicted values.

Figure 7.12 The scaled daily closing price of Lucent incorporated stock for the period November 28, 1998 to November 28, 2003.

Figure 7.13 The comparison between scaled Lucent stock data and the final predicted values.

Figure 7.14 The exchange rate EUR/USD from February 01, 2002 to October 31, 2008.

Figure 7.15 Largest Lyapunov exponent graph for EUR/USD data.

Figure 7.16 The comparison between original EUR/USD data time series and the final predicted values.

Figure 7.17 The daily exchange rates between the US dollar and the Japanese yen for the period January 01, 2003 and December 31, 2006.

Figure 7.18 USD/JPY data in the phase space domain with $D=3$ and $T=5$.

Figure 7.19 USD/JPY data - Comparing the original test data and the predicted values.

Figure 7.20 USD/JPY data - The comparison of the first residual values and the predicted values.

Figure 7.21 The comparison between original USD/JPY data and the final predicted values.

Figure A.1 The original states of Lorenz system.

Figure A.2 Lorenz time series reconstructed into the phase space points with $T=3$ and $D=3$.

Figure B.1 Henon chaotic time series with 1000 data points.

Figure B.2 Power spectrum plot for Henon time series.

Figure B.3 Largest Lyapunov exponent plot for Henon time series.

Figure C.1 Average mutual information graph for Henon time series.

Figure C.2 Cao's graph to identify embedding dimension for Henon time series.

Figure C.3 Henon time series in phase space domain.

Figure D.1 The regression analysis between the feedforward network response and the corresponding targets when applied to Mackey-Glass time series. 


\section{LIST OF NOMENCLATURE/ACRONYMS}

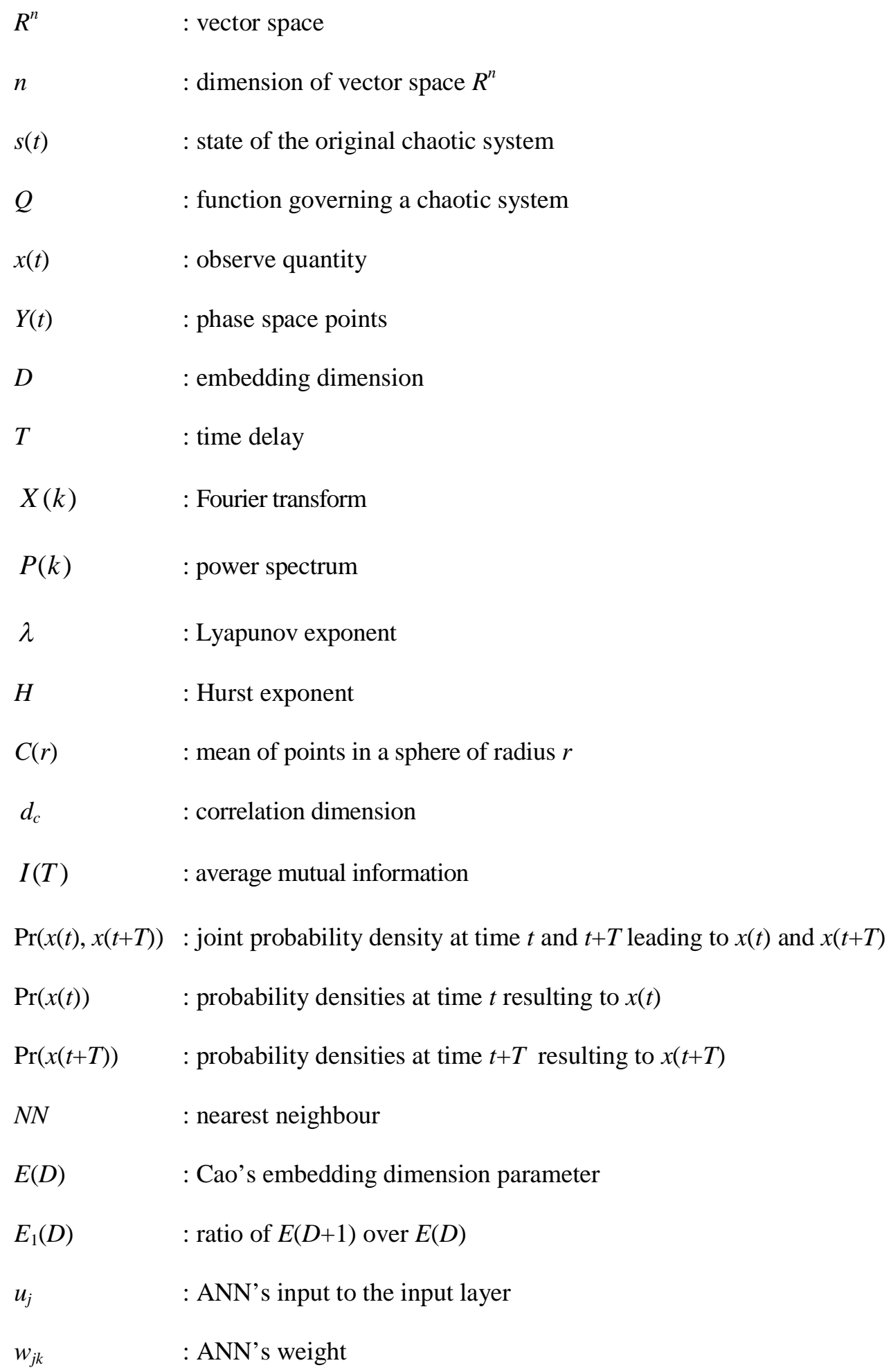


: number of neurons in the hidden layer

: ANN's bias

$r_{k}$

: input to the transfer function $f$

: ANN's transfer function

: ANN's output

: number of neurons in input layer

No

: number of neurons in output layer

$N h$

: number of neurons in hidden layer

$f_{h}(\bullet) \quad$ : hidden layer transfer function

$f_{o}(\bullet) \quad$ : output layer transfer function

$b_{o} \quad$ : output layer bias

$b_{h} \quad:$ hidden layer bias

$w_{h o} \quad:$ hidden layer weight

$w_{\text {ih }} \quad$ : output layer weight

$d_{u} \quad:$ NARX network's input delay

$d_{y} \quad:$ NARX network's output delay.

$w_{i 1 h} \quad:$ weights of the connections between the first input units and the hidden units

$w_{i 2 h} \quad:$ weights of the connections between the second input units and the hidden units

$p_{t} \quad$ : probability of selecting the chromosomes for mating

$f_{t} \quad$ : fitness of the chromosome

$G \quad$ : estimation of function $Q$

$\widehat{y}_{i} \quad:$ predicted data

$\bar{y} \quad$ : average of observed data

$D_{1} \quad$ : the first residual time series embedding dimension 
$T_{1} \quad$ : the first residual time series time delay,

$e_{1}(t) \quad:$ the first residual value

$x^{\prime}(i) \quad$ : prediction value of the time series

$e_{1}^{\prime}(i) \quad:$ prediction value of the first residual time series

$e_{2}(t) \quad:$ the second residual value

$e_{2}^{\prime}(i) \quad$ : prediction value of the second residual time series

$M \quad:$ level of residual analysis

$S / N \quad:$ signal-to-noise ratio

Purelin : linear transfer function

tansig : hyperbolic tangent sigmoid transfer function

traingdx : gradient descent with momentum and adaptive learning rate backpropagation

trainbr : Bayesian regulation backpropagation

trainlm : Levenberg-Marquardt backpropagation

largelyap : function to calculate the largest Lyapunov exponent

takens_estimator: function to calculate correlation dimension

amutual : function to calculate time delay

cao : function to calculate embedding dimension 


\begin{tabular}{|c|c|}
\hline AFS & : Adaptive Fuzzy System \\
\hline AI & : Artificial intelligence \\
\hline AMB & : Adaptive Memory-Based regression \\
\hline ANFIS & : Adaptive-Network-based Fuzzy Interface System \\
\hline ANN & : Artificial Neural Network \\
\hline ANOVA & : Analysis of Variance \\
\hline $\mathrm{AR}$ & : Autoregressive \\
\hline ARMA & : Autoregressive Moving Average \\
\hline ARX & : Autoregressive model with eXogenous input \\
\hline ATNN & : Backpropagation continuous-time FFNN with Adaptable Time delays \\
\hline BGALR & : Breeder Genetic Algorithms with Line Recombination \\
\hline BPNN & : Backpropagation continuous-time FFNN with fixed time delays \\
\hline CCPND & : Complete Counter-Propagation Networks with Delays \\
\hline $\mathrm{CHF} / \mathrm{USD}$ & : Exchange rates between the Swiss franc and the US dollar \\
\hline $\mathrm{CPN}$ & : Counter-Propagation Networks \\
\hline DCS-LMM & : Dynamic Cell Structures combined with Local Linear Models \\
\hline DECS & : Dynamic Evolving Computation System \\
\hline DEM/FRF & : Exchange rates between the Deutsche mark and the French franc \\
\hline DLE & : Distributed Local Experts \\
\hline DLM & : Distributed Local Models \\
\hline DOE & : Design of Experiments \\
\hline EP & : Error Propagation \\
\hline EPNet & : Evolvable Programming Net \\
\hline ESN & : Echo-State Networks \\
\hline EUR/GBP & : Exchange rates between the Euro and the Britain pound \\
\hline EUR/USD & change rates between the Euro and the US dollar \\
\hline
\end{tabular}




\begin{tabular}{|c|c|}
\hline FD & : Fractal Dimension \\
\hline FFNN & : Feedforward Neural Network \\
\hline FIR & : Finite Impulse Response neural network \\
\hline FNT & : Flexible Neural Tree \\
\hline GA & : Genetic Algorithms \\
\hline GDP & : Gross Domestic Product \\
\hline GFPE & : Genetic Fuzzy Predictor Ensemble \\
\hline GMDH-GA & : Group Method of Data Handling using Genetic Algorithms \\
\hline GP & : Gaussian Process \\
\hline GPM & : Genetic Programming-based Modeling \\
\hline HMM & : Hidden Markov Model \\
\hline LLE & : Largest Lyapunov Exponent \\
\hline LoLiMoT & : Locally Linear neuro-fuzzy Model with locally linear model Tree \\
\hline LSTM & : Long Short-Term Memory \\
\hline MANFIS & : Multi-input multi-output Adaptive-Network-based Fuzzy Interface System \\
\hline MAPE & : Mean Absolute Percentage Error \\
\hline MLP & : Multi-Layer Perceptron neural network \\
\hline MSE & : Mean Squared Error \\
\hline MS-SVR & : Multi-Scale Support Vector Regression \\
\hline NARX & : Nonlinear Autoregressive model with eXogenous input \\
\hline NMSE & : Normalized Mean Squared Error \\
\hline $\mathrm{OA}$ & : Orthogonal Arrays \\
\hline OLS & : Ordinary least squares \\
\hline PANN & : Polynomial Artificial Neural Networks \\
\hline PNN & : Probabilistic Neural Networks \\
\hline PRESS & : Predicted Sum of Squares \\
\hline
\end{tabular}




$\begin{array}{ll}\text { RBF } & : \text { Radial Basis Function network } \\ \text { RL-GP } & : \text { Recurrent Linear - Genetic Programming } \\ \text { RMSE } & : \text { Root Mean Squared Error } \\ \text { RNN } & : \text { Recurrent Neural Network } \\ \text { RSOM } & : \text { Recurrent Self-Organizing Map } \\ \text { SOM } & : \text { Self-Organizing Map } \\ \text { SVM } & : \text { Support Vector Machine } \\ \text { SVR } & : \text { Support Vector Regression } \\ \text { TAR } & : \text { Threshold Autoregression } \\ \text { TDNN } & : \text { Time Delay Neural Network } \\ \text { USD/JPY } & : \text { Exchange rates between the US dollar and the Japanese yen } \\ \text { Volterra-TLS } & : \text { Volterra Total Least Square } \\ \text { VQIT } & : \text { Vector-Quantization using Information Theoretic } \\ \text { WNN } & : \text { Wavelet Neural Networks }\end{array}$




\section{CHAPTER 1}

\section{INTRODUCTION}

\subsection{Background and Motivation}

Over the last several decades, prediction of chaotic time series has been a popular and challenging subject. Chaos theory as a new area of mathematics has been used to analyze chaotic systems and draw the hidden information from random-look data produced by chaotic systems. Chaotic time series are deterministic systems and inherit a high degree of complexity. Although chaotic time series show the characteristics of dynamical systems as random, in the embedding phase space, they present deterministic behaviour [1].

The application of chaos theory is becoming increasingly widespread in a variety of disciplines. An early application of chaos theory was proposed in modelling and data analysis of mixing processes $[2,3]$. Chaos theory has been applied to the control of robot servos where the robot can learn from its environment and recognize the beginning of random behaviour [4]. Chaos has also been applied in communications, the optimization of telephone exchanges [5] and the transmission of digital signals [6]. Data analysis methods developed for the analysis of chaotic behaviour have been applied to bulk chemical reactions [7].

Prediction of nonlinear time series is a useful method to evaluate characteristics of dynamical systems. Prediction of chaotic time series has been observed in the areas of marketing system [8,9], foreign exchange rates [10], signal processing [11], supply chain 
management [12], traffic flow [13], power load [14], weather forecasting [15], sunspot [16], cardiovascular control [17] and many others. Due to the importance of these fields and widespread applications of chaotic systems in real life, the interest in a robust technique to predict chaotic time series has increased.

There are several types of time series such as periodic, seasonal, stochastic, chaotic and others. To differentiate between deterministic and stochastic data, it is necessary to search for similar or nearby states and compare their progress over time. Determining the difference between the time progress of the nearby states, the random data can be differentiated from deterministic data. For deterministic data the error will remain either small for stable data or evolve exponentially with time for chaotic data. The error for stochastic data will be randomly distributed [18]. In this study, the focus is to identify a chaotic time series from non-chaotic data and forecast the future of the chaotic time series.

Many of the proposed forecasting methods in the literature are either ineffective when applied to chaotic time series or difficult to implement. The motivation of this dissertation is to develop a more easy-to-use, effective in terms of error indices and practical method to forecast chaotic time series.

\subsection{Overview of Chaos Theory and Chaotic Time Series Forecasting}

Chaos theory, as an essential part of nonlinear theory, has provided an appropriate tool to study the characteristics of the dynamical systems and predict the trend of complex systems. There are four fundamental characteristics for chaotic systems: aperiodic, that is, the same state will not be repeated; bounded, meaning that neighbouring states remain within a finite range and do not approach infinity; 
deterministic, meaning that there is a governing rule with no random term to predict the future states of the system; and sensitivity to initial conditions, meaning that small difference in initial conditions will cause two points close to each other to diverge as the state of system progresses [19].

Takens' embedding theorem [20] is an essential element of chaotic time series analysis. A set of single observations from a chaotic system can be reconstructed into a series of $D$-dimensional vectors with two parameters of time delay and dimension. Based on Takens' theorem, if the value of embedding dimension is large enough, the reconstructed vectors exhibit many of the significant properties of the original time series [21].

A number of techniques have been introduced in the last several decades to predict chaotic time series. These methods are classified into two major categories of local and global modelling methods. In the local modelling technique, a group of local estimators performs the forecasting task. In global modelling, a single fitting model predicts the future of a system [22,23]. Local modelling methods have been proposed by Farmer and Sidorowich [24], Kennel [11] and McNames [25] among others.

Artificial neural networks (ANNs) as part of global modelling were employed by researchers to forecast chaotic time series. Multi-layer perceptron neural networks (MLP) $[16,26]$, recurrent neural networks (RNN) [1,27], the nonlinear autoregressive model with exogenous input (NARX) [28,29] are applied to chaotic time series.

Some other artificial intelligence (AI) methods such as radial basis function (RBF) [30,31], self-organizing map (SOM) [32,33], support vector machine (SVM) [34,35], genetic algorithms (GA) [36,37], fuzzy, neuro-fuzzy [38,39] and wavelet neural networks (WNN) $[40,41]$ among others are used in the literature to forecast chaotic time series. 
A combination of forecasting methods forms another type of forecasting technique known as ensemble modelling. The research work presented by Wichard and Ogorzalek [42] describes the use of ensemble methods to build proper models for chaotic time series prediction.

Taguchi's design of experiments (DOE) method has been adopted by many researchers in the field of artificial intelligent to find an optimum design. Kim and Yum [43], Khaw et al. [44], Lin and Tseng [45], Ortiz-Rodríguez et al. [46], Packianather and Drake [47], Wang et al. [48], Yang and Lee [49], Peterson et al. [50] have used Taguchi's DOE to optimize the design of ANN. Mouli et al. [51] have demonstrated that Taguchi's method can be utilized as a pre-step to the neural network method to obtain the optimum result.

\subsection{Research Objective}

The main objective of this dissertation is to develop more accurate and practical method to forecast chaotic time series. It is essential to integrate an easy to implement methodology with proper use of the theories to ensure the resulting forecasting method is capable of predicting chaotic time series more accurately and effectively. The following steps are taken into consideration to achieve the objective of this research work:

- Developing an effective and practical forecasting method based on the precise definition of chaos theory and embedding theorem;

- Incorporating the residuals of the forecasting method in order to increase the forecasting accuracy; 
- Enhancing the design of the proposed forecasting method using a systematic approach to facilitate the combination selection of ensemble artificial neural networks and their parameters;

- Applying the proposed forecasting method to well-known chaotic time series and comparing its performance with the results reported in the literature;

- Applying the proposed forecasting method to the financial time series, namely, stock markets and foreign exchange rates.

\subsection{Dissertation Overview}

The block diagram shown in Figure 1.1 presents the proposed methodology to develop an original model to forecast chaotic time series. The block diagram is divided into two Blocks, A and B. Block A demonstrates the process implemented in Chapter 4. In Chapter 4, a unique technique based on precise definition of chaos theory and artificial neural networks is proposed to analyze and forecast chaotic time series. As shown in Figure 1.1, the first step after selecting a time series is to analyze the time series and investigate the presence of chaos in the time series. When the presence of chaos in confirmed, then the embedding parameters, $D$ and $T$, are determined and accordingly the phase space points are formed. Based on chaos theory, there exists an unknown mathematical equation which can forecast the future value of the phase space points. An artificial neural network is employed to capture the functional relationships among the given phase space points. 


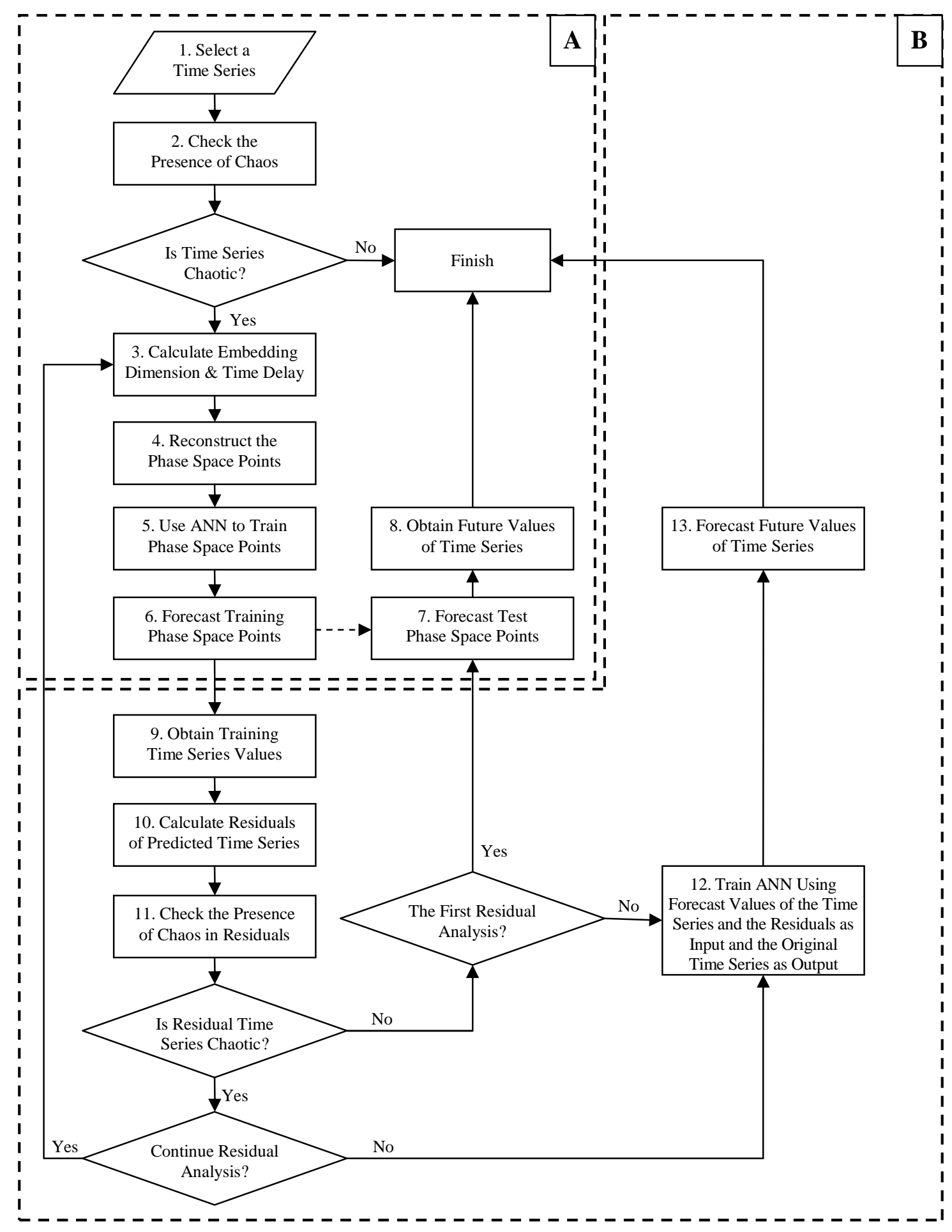

Figure 1.1 A block diagram demonstrating the proposed methodology. 
Therefore, the embedded phase space points are fed into a neural network and trained. The weights and biases of the trained neural network are kept to forecast the future of phase space points. When the unknown phase space points are predicted, the future values of the time series are obtained consequently. Genetic algorithms can be applied to block A to improve the prediction results by selecting proper initial weights and biases for the artificial neural network. Block $\mathrm{A}$ in Figure 1.1 can be used independently to forecast one step ahead of a chaotic time series.

Block B in Figure 1.1 is added to the proposed forecasting method to increase accuracy of prediction by investigating and incorporating the residuals. This will be discussed in Chapter 5. As demonstrated in Block B Figure 1.1, the residuals of the predicted time series are further analyzed. In some events, the residuals show a high degree of correlation and demonstrate chaotic behaviour. Therefore, the residuals are considered as a new chaotic time series and reconstructed according to the embedding theorem. A new neural network is trained to predict the future values of residual time series. The residual analysis can be repeated several times. The number of residual analysis depends on the characteristics of the residuals and if the incorporation of the residuals can enhance the performance of the forecasting method. Finally, a neural network is trained using neural networks' result values of the time series and the residuals as input and the original time series as output. The weights and biases of the final artificial neural network are kept to predict the future values of the original time series. Taguchi's design of experiments can be applied to the overall process illustrated in Figure 1.1 to identify the proper factor-level combinations and consequently improve the forecasting results. The implementation of Taguchi's design of experiments on the proposed forecasting method is discussed in Chapter 6 . 


\subsection{Dissertation Outline}

This dissertation consists of eight chapters. The current chapter presents an introduction to chaos theory and the background of chaotic time series forecasting. The motivation and objective of this study are discussed in this chapter.

Chapter 2 provides a comprehensive literature review on chaos theory and different types of chaotic time series forecasting such as local, global and ensemble methods.

Chapter 3 defines chaos theory mathematically and discusses the methods to determine existence of chaos in times series. The methods to calculate embedding parameters and reconstruct chaotic time series are presented in Chapter 3. Static and dynamic artificial neural networks, and genetic algorithms are formulated in this chapter.

Chapter 4 applies the precise definition of chaos theory and the embedding theorem to develop an original technique to forecast chaotic time series. Artificial neural networks and genetic algorithms are adopted to develop the forecasting method.

In Chapter 5, the application of residual analysis and ensemble neural networks in increasing the accuracy of the proposed forecasting method are demonstrated. Dynamic ANNs are utilized to improve the performance of the proposed forecasting method.

Taguchi's design of experiments as an efficient approach to identify the proper factor-level combinations is introduced in Chapter 6 to improve the result of the proposed forecasting method for chaotic time series.

Chapter 7 applies the forecasting method developed in this study to real life applications such as stock markets and foreign exchange rates.

Chapter 8 concludes the objective of the current research work and proposes future work in this area. 


\section{CHAPTER 2}

\section{LITERATURE REVIEW}

\subsection{Chaos and Chaos Theory}

In 1686, Sir Issac Newton in "Mathematical Principles of Natural Philosophy" revealed a new finding that almost every phenomenon in universe works under deterministic equations with no element of chance and can be fully predicted [52,53].

In 1779, Pierre Simon De Laplace confirmed Newton's theory and added that due to errors in observations random terms need to be introduced to the governing equations $[54,55]$. Further, he proposed that the current state in nature is the result of the state in the preceding moment.

A century later, in 1873, Clerk Maxwell said, "when an infinitely small variation in the present state may bring about a finite difference in the state of the system in a finite time, the condition of the system is said to be unstable...[and] renders impossible the prediction of future events...”[56].

In 1898, the French mathematician, Jacques Hadamard, highlighted that a very small difference or error in initial conditions can make the long-term prediction of a system impossible [57].

In the early 20th century, Henri Poincare stated that in certain deterministic systems a subtle change which is negligible can cause a considerable effect which cannot be neglected. He stated that due to this phenomenon, the outcome of the deterministic 
system appears as random. Based on his theory, in 1903, Poincare suggested that if the laws of nature and the initial state of the universe were known exactly, the future state of the universe could be predicted. Even the approximation of the initial state of the universe could not be helpful as a subtle difference in the initial state would cause a considerable difference in the future state of the universe [55].

In the early 1960s, Edward Lorenz created a computer model using 12 deterministic equations to forecast weather systems reasonably accurately. In 1961, he repeated a simulation by changing the number of decimals in the initial conditions of a particular model. He realized that the small change in the initial conditions caused a drastic difference in the final result and exponentially amplified the error [58]. This unusual behaviour of deterministic systems was recognized for the first time by Lorenz and he developed the well-known butterfly effect. He concluded that the sensitivity to initial conditions in certain systems amplifies a small error due to a subtle change in initial conditions and causes enormous consequences. He analogically stated that a small change in the state of the atmosphere due to a flap of a butterfly's wings can grow exponentially and over a period of time can create a devastating tornado.

Later in 1975, Li and Yorke [59] used the term chaos for the first time. The phenomena which $\mathrm{Li}$ and Yorke described in their paper as chaos was the same phenomena that Newton, Laplace, Poincare and Lorenz had previously studied.

In 1986 at a Royal Society seminar the word chaos was defined as "stochastic behaviour occurring in a deterministic system" [54]. Ian Stewart [54] interpreted the definition accepted at the Royal Society seminar and suggested "Chaos is lawless behaviour governed entirely by law". 
In 1993, Ralph Stacey [60] focused on the patterns created by chaotic systems and defined chaos as a pattern generated by a fixed law within a random behaviour.

Two years later, Kaplan and Glass [61] elaborated the definition of chaos and suggested "chaos is defined to be aperiodic bounded dynamics in a deterministic system with sensitive dependence on initial conditions".

In 1996, Abarbanel [62] elaborated on Kaplan and Glass's definition and proposed that "chaos is the deterministic evolution of a nonlinear system which is between regular behaviour and stochastic behaviour or 'noise'. This motion of nonlinear systems is slightly predictable, non-periodic and specific orbits change exponentially rapidly in responses to changes in initial conditions or orbit perturbations".

Being aperiodic and bounded, having the sensitivity to initial conditions and possessing a prediction horizon due to the deterministic nature of chaotic systems are the four key points highlighted in Kaplan and Glass [61] and Abarbanel's [62] definition.

\subsection{Chaotic Time Series Forecasting}

The future state of a complex system is not known to anyone; however, the attempt to approximately predict the future state is beneficial to decision makers. In the past several decades, some nonlinear techniques have been introduced in the literature to forecast the future state of chaotic systems.

Before the 1980s, traditional linear autoregressive moving average (ARMA) models introduced by Box and Jenkins [63] were popular models in the area of forecasting [29,64]. ARMA models are linear and are not capable of forecasting nonlinear, non-stationary and chaotic time series $[29,31,65,66,67]$. Therefore, ARMA 
models are not popular, accurate and practical methods to forecast nonlinear time series $[68,69,70,71,72,73]$. In the 1980 s, gradually the essential tools to analyze chaotic time series were developed [74]. Chaos theory, as an essential part of nonlinear theory, has provided an appropriate tool to illustrate the characteristics of the dynamical systems and predict the trend of complex systems.

There are two main approaches to forecast chaotic time series: local modeling and global modeling [23,75,76]. A combination of forecasting methods known as the ensemble method is also used for forecasting.

\subsubsection{Local Models}

Local models perform the forecasting by searching for the local regions of the time series which approximately present a region of the data immediately before the point to be forecasted. In local modeling, the overall prediction model consists of several local estimators where the local estimators define the various portions of the input space [22].

Several local methods are presented in the literature to forecast chaotic time series. In 1969, Lorenz [77] proposed the first local model to predict the weather system. In 1987, Farmer and Sidorowich [24] developed a local model using scaling law. Later, Casdagli [23] proposed a new technique based on Farmer and Sidorowich's [24] scaling law.

In early 1990s, Jacobs et al. [78] and Kennel [11] employed local models to forecast chaotic time series. In late 1990s, McNames [25] proposed a nearest trajectory strategy which later was followed by Bontempi et al. [76] to develop a predicted sum of squares (PRESS) method. 
In 1998, Zhang et al. [79] developed a local forecasting model based on Lyapunov exponents. In the same year, a method involving dynamic cell structures combined with local linear models (DCS-LMM) was reported by Chudy and Farkas [80] and ordinary least squares (OLS) by Kugiumtzis et al. [81].

In 2001, Sello [82] developed a model based on a local hypothesis of the behaviour of embedding space, employing an optimal number of neighbouring vectors. One year later, Fontenla-Romero [83] proposed distributed local models (DLM). Recently, Gholipour et al. [84] presented a locally linear neuro-fuzzy model with locally linear model tree (LoLiMoT) and Martinez-Rego et al. [22] reported distributed local experts based on vector-quantization using information-theoretic concept (DLE-VQIT).

\subsubsection{Global Models}

There are a number of forecasting methods based on the global modeling approach in the literature. In global models only one fitting function is engaged to forecast the future of the system [33]. Since the 1970s, numerous global methods such as bilinear models [85,86], exponential autoregressive models [87,88], state-dependent models $[89,90]$, threshold autoregression (TAR) [91], the threshold model [92], neural gas [93], adaptive memory-based regression (AMB) [94], the long short-term memory (LSTM) Gaussian process (GP) [95], echo-state networks (ESNs) [96], the flexible neural tree (FNT) [97], and the dynamic evolving computation system (DECS) [98] are introduced in the literature.

Artificial neural networks (ANNs) as part of global modeling were employed by researchers to forecast chaotic time series. Multi-layer perceptron neural networks (MLP) has been used by Elsner [99], Park et al. [16] and Liu et al. [26]. Tapped delay line neural 
networks (TDLNN) are employed by Veelen et al. [100] and Moller [101]. Zhang and Man [1], Ma et al. [27], Tenti [102], Connor and Atlas [103], Connor and Martin [104], Bone et al. [105], Han et al. [106], Assaad et al. [107] have utilized recurrent neural networks (RNN). The nonlinear autoregressive model with exogenous input (NARX) has been also applied to chaotic time series forecasting by Menezes and Barreto [28] and Diaconescu [29].

The ensemble method is introduced to improve the result of forecasting by merging individual predictors. An ensemble can be the combination of the same class of models such as ANN, SOM, SVR or different types [42]. The early ensemble model was introduced by Guan et al. [108] which combined the nearest neighbours, artificial neural networks, and genetic algorithms. Later, Lin et al. [109] merged the nearest neighbours technique with genetic algorithms.

Chaotic time series forecasting using radial basis functions (RBF) [30,110,111, $112,113,114]$, the RBF based adaptive fuzzy system [115], the pseudo-Gaussian RBF [116], the normalized RBF [112], the RBF autoregressive model (RBF-AR) [117,118] and the RBF autoregressive model with exogenous input (RBF-ARX) $[119,120,121]$ are observed in the literature. Wavelet neural networks (WNN) $[40,41,122,123,124]$ and multi-wavelet networks [125] are also used to forecast chaotic time series.

The finite impulse response neural network (FIR) [126,127], backpropagation continuous-time feedforward neural networks with fixed time delays (BPNN) [128], backpropagation continuous-time feedforward neural networks with adaptable time delays (ATNN) [128], the evolvable programming net (EPNet) [129], counterpropagation networks (CPN) [130], complete counter-propagation networks with delays 
(CCPND) [131] and polynomial artificial neural networks (PANN) [132] are examples of global models applied to complex systems.

Support vector regression (SVR) $[133,134,135,136]$ and its variations such as multi-scale support vector regression (MS-SVR) [137], self-organizing map (SOM) $[32,33,138,139,140,141]$ and its variations such as recurrent SOM (RSOM) [32] and support vector machine (SVM) $[34,68,142]$ and its variations such as support vector echo-state machine [143] and least square SVM [35] have been used for chaotic time series prediction.

Some other artificial intelligence (AI) methods such as fuzzy and neuro-fuzzy [38,39,144], adaptive-network-based fuzzy interface system (ANFIS) [145], multi-input multi-output adaptive-network-based fuzzy inference system (MANFIS) [39], genetic algorithms [36,37,108,109], breeder genetic algorithms with line recombination (BGALR) [146], group method of data handling using genetic algorithms (GMDH-GA) [147], genetic programming-based modeling (GPM) [69], recurrent linear - genetic programming (RL-GP) [148], genetic programming augmented by binary string fitness [149], volterra [150,151,152] and volterra total least square (Volterra-TLS) [153] have been developed to forecast dynamical systems. Genetic fuzzy predictor ensemble (GFPE) [154], error propagation (EP) [155], ensemble self generating neural networks merged with genetic algorithms [156] come under ensemble forecasting methods. Wichard, and Ogorzalek [42], Rojas [31] and Bouchachia and Bouchachia [157] reported ensemble models which are capable of forecasting chaotic time series. 


\subsection{Summary}

Since the 1980s, the necessary tools to study and forecast chaotic time series have been developed. In local modeling, the overall prediction model consists of several local estimators where the local estimators define the various portions of the input space. In global modeling, one fitting function is engaged to forecast the future of the system. The ensemble method is the combination of individual predictors.

Based on the definition of global modeling and the ensemble method, an integrated definition for the ensemble-global model is a combination of predictors or forecasting methods which form an overall fitting function to forecast the future of the system. In Chapter 4, a global forecasting model is developed to forecast chaotic time series. In Chapter 5, an ensemble-global model is introduced to increase the accuracy of prediction. In Chapter 6, the result of the ensemble-global model proposed in Chapter 5 is improved with Taguchi's DOE. The application of the improved ensemble-global model proposed in this study is applied to stock markets and foreign exchange rates in Chapter 7. 


\section{CHAPTER 3}

\section{AN OVERVIEW OF THE EMBEDDING THEOREM AND ARTIFICIAL INTELLIGENCE}

There are four fundamental characteristics for chaotic systems: aperiodic, that is the same state will not be repeated; bounded, meaning that neighbouring states remain within a finite range and do not approach infinity; deterministic, meaning that there is a governing rule with no random term to predict the future state of the system; and sensitivity to initial conditions, meaning that a small difference in initial conditions will cause two states close to each other to diverge as the state of the system progresses. Quasi-periodic and hyper-chaotic systems fall under chaotic system [19]. Nonlinear feedback systems, in which the current state of the system is fed back to generate the future state, have the potential of being chaotic $[60,158]$. In the next section, the characteristics of chaotic systems are reviewed in more detail.

The purpose of this chapter is to present the necessary theories and tools adopted in this dissertation to develop an original method to forecast chaotic time series. In Section 3.1, the characteristics of chaotic systems are discussed. Chaos theory as an essential theory to properly structure a forecasting method for chaotic systems is introduced in Section 3.2. The definition of time series and chaotic time series, the methods to detect the existence of chaos in a give time series, determining embedding parameters and the embedding theorem are the subsections of Section 3.2. Two classes of artificial intelligence namely, artificial 
neural networks and genetic algorithm are discussed in this chapter. Artificial neural networks as practical tools to find a relationships among given data are elaborated in Section 3.3. Genetic algorithms as an evolutionary method used for optimization are discussed in Section 3.4. This chapter is concluded in Section 3.5.

\subsection{Characteristics of Chaotic Systems}

It is critical to identify the characteristics of chaotic systems precisely to choose proper tools to analyze and investigate dynamical systems. Wilding [19] has presented a comprehensive review on characteristics of chaotic systems.

Chaotic systems are deterministic. A system is deterministic when a governing law determines the future state of the system based on previous states with no involvement of random terms [159].

Complex systems inherit bounded behaviour. When data points which are extracted from a dynamical system remain within a finite range and do not approach infinity, the system is bounded. Another requirement of a bounded system is being stationary. Being stationary means to have similar behaviour at all times or the mean and standard deviation stay constant [61].

Aperiodic behaviour is the key element of chaotic systems. An aperiodic characteristic is defined as a property of a system to show irregular oscillations which do not increase, decrease or stay steady. In other words, the system is neither random nor periodic [61]. 
A system which is operating in a stable mode can change to other modes such as periodic or chaotic, if the system's parameters are changed. Also, it is common for complex systems to switch between various modes as the system progresses over time [160].

Sensitivity to initial conditions is the most well-known characteristic of chaotic systems [58]. Even a subtle difference in initial conditions amplifies over time until the future states of two points initially close to each other become drastically different. This critical property of chaotic systems makes the long term prediction of complex systems impossible. The Lyapunov exponent is an appropriate tool to quantify the convergence or divergence of the system and determine the predictability horizon [161].

Chaotic systems generate patterns despite random-look behaviour. The generated patterns do not repeat themselves but they have similar properties. Snowflakes are an example of these types of patterns. A deterministic system generates the shape of snowflakes; however, a small change impacts the final shape of the snowflakes. In chaos literature, the attractor is referred to as the patterns produced by chaotic systems [19].

The term attractor is defined by Crutchfield et al. [55] as "The set of points in phase space visited by the solution to an evolution equation long after (initial) transients have died out". In other words, attractors geometrically form the long-term projection of a system in the phase space mode [55]. For a stable system, the attractor is a fixed point attractor. The attractor for periodic systems is cyclic. A quasi-periodic system has an attractor which is a combination of two or more periods. Chaotic systems have a strange attractor [162] which is described by a stretching and folding operation [55]. The stretching and folding process produces patterns which are known as fractals. 
The view of reductionism is not valid for analyzing chaotic systems. Reductionism states that a complicated problem can be broken into simple portions [163]. Later, by analyzing the simple portions of the complicated problem, the overall problem can be resolved. Goldratt [164] demonstrated that in chaotic systems this methodology is not valid and a subtle change in any of the simple parts may result in a drastic change in the overall system's response.

Chaotic systems when analyzed by a computer can produce different results. An identical code can produce completely different results when run with two different computers or software [54]. Peitgen [58] warned of the risk of relying only on the result of computer packages. He emphasized that it is necessary to understand the outcome of the computer packages and the reasons behind the generated outcomes.

\subsection{Theory of Chaos}

Chaos theory is a new area of mathematics which is also known as complexity or nonlinear dynamics [165]. Although chaotic systems are deterministic and governed by certain laws, due to nonlinear effects and sensitivity to initial conditions they are predictable within limited horizon [54].

Many natural systems show nonlinear or chaotic behaviour. Using chaos theory, these systems have been described by mathematical equations. For a chaotic system, the phase space is defined as a vector space $R^{n}$ with each point in the phase space being described by an $n$-dimensional vector $s(t)$, which is required to obtain the progression of the system [166]. $s(t)$ is defined in Eq. (1):

$s(t)=\left[s_{1}(t), s_{2}(t), s_{3}(t), \ldots, s_{n}(t)\right]$ 
where $t$ is an index for the time series and $n$ is the dimension of vector space $R^{n}$. With the use of the nonlinear function $Q: R^{n} \rightarrow R^{n}$, which describes the system, the future value of the system at time $t+\tau$ can be determined by Eq. (2).

$$
s(t) \rightarrow Q(s(t))=s(t+\tau)
$$

A small change in the state of the system, $s(t)$, will substantially influence the trend of the system and after several iterations, the system becomes unforeseeable. This behaviour of dynamical systems is known as sensitivity to initial conditions or the butterfly effect [21].

The progression of a non-random system creates a trajectory named an attractor. Takens' embedding theorem [20] states that because the value of $s(t)$ and its components, $s_{1}(t), s_{2}(t), s_{3}(t), \ldots$ in a chaotic system are unknown, if one is able to observe a single quantity or variable $x(t)$ from this dynamical system, then the attractor can be unfolded from this set of observed samples [62]. This means that if a single quantity $x(t)$ is observed from a chaotic system, the reconstructed dynamics of a system $Y(t)=[x(t)$, $x(t+T), x(t+2 T) \ldots]$, with $T$ defined as time delay, is geometrically similar to the original attractor. Therefore, if a dynamical system $s(t) \rightarrow s(t+1)$ exists, then sequential order of reconstructed phase space points $Y(t) \rightarrow Y(t+1)$ follows the unknown dynamics of $s(t) \rightarrow \mathrm{s}(t+1)$. Therefore, the behaviour of the actual system is reflected in the observed time series generated from the system [154]. More details on chaos theory can be found in the text book by Abarbanel [62]. A numerical example to explain chaos theory and Taken's embedding theorem is presented in Appendix A. 


\subsubsection{Chaotic Time Series}

A time series is a sequence of scalar values over time. Zhang et al. [39] describe a time series as "a sequence of regularly sampled quantities out of an observed system". Kantz and Scheiber [167] define a time series as a set of scalar values which measures the status of a certain system over time. A time series is the historical record of a system, with the measurements taken at regular intervals with a consistency in the method of measurement and the system [168]. It is a useful source of information to analyze and investigate the characteristics and behaviours of a system [39]. Being aperiodic, bounded, deterministic and sensitive to initial conditions differentiates chaotic time series from other types of time series [19]. Time series analysis consists of the techniques to manipulate, characterize and perform quantitative and qualitative analysis to understand the underlying characteristics of a system [168].

\subsubsection{Determining Chaos in Systems}

In analyzing time series, an important step is to determine the characteristics of the data. The following methods have been used to differentiate chaotic data from non-chaotic data. The Lyapunov exponent method which looks for the main characteristics of chaotic systems, sensitivity to initial conditions, is the most preferable method in the literature.

\section{Fourier transform}

Fourier transform can be used to identify chaos in a given time series. The Fourier transform calculates the present frequencies in a time series. Fourier transform of the time series, $x(t), t=0,1,2, \ldots, N-1$, where $N$ is the number of points can be calculated by Eq. (3):

$$
X(k)=\frac{1}{N} \sum_{t=0}^{N-1} x(t) e^{-2 \pi i(k t / N)} \quad k=0,1,2, \ldots, N-1
$$


where $i$ is the imaginary number [169].

The power spectrum is the square of the $X(k)$ and can be calculated by Eq. (4).

$P(k)=\frac{1}{N^{2}}\left|\sum_{t=0}^{N-1} x(t) e^{-2 \pi i(k t / N)}\right|^{2} \quad k=0,1,2, \ldots, N-1$

Plotting the power spectrum of the time series can assist in determining the nature of the time series. The spectrum for chaotic time series will be broadband, meaning that the difference between the highest power value and the lowest value in the power spectrum plot is considerable, with a broad peak, meaning that a wide range of frequencies are available in the power spectrum plot. Random data should have a constant valued power spectrum. For periodic data, the power spectrum spikes at frequencies that characterize the system and remain close to zero for the others $[21,170]$. A numerical example is included in Appendix B.

\section{Lyapunov exponent}

An important characteristic of chaotic systems which is defined as the butterfly effect is the high sensitivity of the system to the initial conditions [161]. The largest Lyapunov exponent is the most practical method to identify chaotic behaviour in a system. The Lyapunov exponent quantifies the convergence and divergence of neighbouring trajectories. If there exists a function which maps $x(t)$ to $x(t+1), x(t) \rightarrow R(x(t))=x(t+1)$, for two nearby initial points at $x(0)$ and $x(0)+\Delta x(0)$, after one iteration the separation of the points can be calculated by Eq. (5):

$\Delta x(1)=R(x(0)+\Delta x(0))-R(x(0)) \approx \Delta x(0) R^{\prime}(x(0))$

where $R^{\prime}=d R / d x$. 
The Lyapunov exponent at $x(0)$ can be defined such that $e^{\lambda}=|\Delta x(1) / \Delta x(0)|$ or can be rearranged as shown in Eq. (6).

$\lambda=\ln |\Delta x(1) / \Delta x(0)| \approx \ln \left|R^{\prime}(x(0))\right|$

The quantity $|\Delta x(1) / \Delta x(0)|$ is the Lyapunov exponent or the measurement of the stretching at $x=x(0)$. If $\Delta x(1) / \Delta x(0)$ is negative, it means the two nearby points interchange their order (the larger becomes smaller, and vice versa) upon iteration.

To obtain the global Lyapunov exponent, the average of Eq. (6) over a large number of iterations is calculated as presented in Eq. (7) [171].

$\lambda=\lim _{N \rightarrow \infty} \frac{1}{N} \sum_{t=0}^{N-1} \ln \left|R^{\prime}(x(t))\right| \quad t=0,1,2, \ldots, N-1$

The value zero is interpreted as cyclic behaviour, a negative exponent means nonchaotic behaviour, and a positive Lyapunov exponent proves the existence of chaos in the system [21,161]. A numerical example is included in Appendix B.

\section{Hurst exponent}

Harold Edwin Hurst is known for introducing the Hurst exponent as a measurement for the predictability of a time series [172,173]. The Hurst exponent is determined using R/S analysis. For a given time series with $N$ points and selected $p$ where $10 \leq p<N / 2$, the times series can be divided into $N / p$ blocks.

For each block the maximum range, the average value and the standard deviation are calculated. Then for each block the value of (range)/(standard deviation), $r s$, is obtained and averaged over all the blocks. Eq. (8) shows the relation between the average value $r s$ and Hurst exponent: 
$r s=\left(\frac{p}{2}\right)^{H}$

where $H$ is the Hurst exponent.

Hurst exponents can change between 0 and 1 . The Hurst exponent of 0.5 shows a true random walk. A value between 0 and 0.5 indicates non-persistent behaviour, meaning that the data is not random but the current trend is unlikely to continue. A Hurst exponent between 0.5 and 1 proves that the data are more persistent and the current direction is likely to continue. A Hurst exponent of 0 means that the time series changes direction with every sample. A constant time series with non-zero gradient will result in a Hurst value of 1 [174]. A numerical example is included in Appendix B.

\section{Fractal dimension}

Another method to identify the existence of chaos in a system is fractal dimension. Non-integer fractal dimension shows that the system is chaotic. Correlation dimension is one of the most common fractal dimensions used in the literature [21,175]. If a sphere of radius $r$ is centred on a specific point in $D$-dimensional space, then the mean of points in the sphere, $C(r)$, excluding the centre point can be calculated by Eq. (9):

$$
C(r)=\frac{1}{N(N-1)} \sum_{j=0}^{(N-D T-1)} \sum_{k=0, k \neq j}^{(N-D T-1)} \Theta(r-|Y(j)-Y(k)|)
$$

where $\Theta(x)$ is the indicator function defined as $\Theta(x)=\left\{\begin{array}{lll}0 & \text { if } & x<0 \\ 1 & \text { if } & x \geq 0\end{array}\right.$.

A plot of $C(r)$ versus $r$ should give an approximately straight line whose slope is the correlation dimension $d_{c}$ as formulated by Eq. (10). 
$d_{c}=\lim _{R \rightarrow 0} \frac{d \log C(r)}{d \log r}$

The correlation dimension $d_{c}$ with integer value shows that the attractor is a simple geometric object. A non-integer value for $d_{c}$ indicates that the attractor is strange and the system is chaotic [21,175]. A numerical example is included in Appendix B.

\subsubsection{Embedding Theorem}

In the absence of a governing equation for a chaotic system, the phase space points are generated from the original time series using the embedding method [166]. The embedding theorem proposes that if an appropriate dimension is selected for a chaotic time series, the data can be reconstructed and the unfolded data can reveal the hidden information of the system.

A chaotic time series can be embedded in multi-dimensional space by plotting the data point $Y(t), t=1,2, \ldots, N-(D-1) T$ where $Y(t)=[x(t), x(t+T), \ldots, x(t+(D-1) T)], N$ is the length of the original time series, $D$ is the embedding dimension of the time series, and $T$ is time delay. The method of embedding observed data in a $D$-dimensional space is known as phase space reconstruction [62]. To apply Taken's theorem properly, appropriate selection of the embedding dimension $D$ and time delay $T$ are required [21]. A numerical example is included in Appendix C.

\subsubsection{Determining Time Delay}

To select proper time delay $T$, low correlation between nearby elements in the phase space points should be provided without being too long. The first minimum of the average mutual information function can be selected as time delay [21]. 
Average mutual information is a theoretic method to connect two sets of measurements with each other and establish a criterion for their mutual dependence based on the notion of information connection between them. Average mutual information is used to give a precise definition to the notion that measurements $x(t)$ at time $t$ are connected in an information theoretic fashion to measurements $x(t+T)$ at time $t+T$. Therefore, the average mutual information between $x(t)$ and $x(t+T)$ can be calculated by Eq. (11):

$I(T)=\sum_{x(t), x(t+T)} \operatorname{Pr}(x(t), x(t+T)) \log _{2}\left[\frac{\operatorname{Pr}(x(t), x(t+T))}{\operatorname{Pr}(x(t)) \operatorname{Pr}(x(t+T))}\right]$

where $\operatorname{Pr}(x(t), x(t+T))$ is the joint probability density at time $t$ and $t+T$ leading to $x(t)$ and $x(t+T)$ concurrently. $\operatorname{Pr}(x(t))$ and $\operatorname{Pr}(x(t+T))$ are the probability densities at time $t$ and $t+T$ resulting in $x(t)$ and $x(t+T)$, respectively. For a time series, the histogram of the measurements can be used to calculate the probabilities [21,62].

$I(T)$ determines the average amount of information shared by two values in the time series. When the value of $T$ increases, $x(t)$ and $x(t+T)$ become independent and $I(T)$ will tend to zero [62]. A numerical example is included in Appendix C.

\subsubsection{Determining Embedding Dimension}

To choose a suitable embedding dimension $D$, it is important to understand the impact of the embedding dimension on the structure of an attractor. As the embedding dimension increases, the attractor unfolds. When the attractor is unfolded completely, the same point on the attractor will not cross itself [21].

The method of false nearest neighbours suggests that, when the attractor's trajectories come to cross, two neighbouring phase space points stand extremely apart from each other in the successive order of the embedded time series. Cao [176] has 
implemented this idea and has developed a method to estimate an effective embedding dimension [21]. Cao [176] defined Eq. (12) to calculate $E(D)$ :

$E(D)=\frac{1}{N-D T} \sum_{t=0}^{N-D T-1} \frac{\left\|Y_{D+1}(t)-Y_{D+1}^{N N}(t)\right\|}{\left\|Y_{D}(t)-Y_{D}^{N N}(t)\right\|}$

where $N$ is the length of the original time series, the subscript $D$ refers to the embedding dimension, and the superscript $N N$ means the nearest neighbour to the other vector as calculated by Eq. (13).

$\left\|Y_{D}(t)-Y_{D}^{N N}(t)\right\|=\max _{0 \leq j \leq D-1}\left|x(t+j T)-x^{N N}(t+T)\right|$

As $D$ becomes large enough, $E_{1}(D)$, the ratio of $E(D+1)$ over $E(D)$, which can be calculated by Eq. (14) approaches 1.

$E_{1}(D)=\frac{E(D+1)}{E(D)}$

The appropriate embedding dimension is given by the value of $D$ where $E_{1}(D)$ stops changing. Practically, this means choosing the value of $D$ where $E_{1}(D)$ begins to level out, near unity [21]. A numerical example is included in Appendix C.

\subsection{Artificial Neural Networks}

Artificial neural networks (ANNs) are nonlinear methods which simulate the human neural system. They have the functions of self-organizing, data-driven, self-study, self-adaptive and associated memory. ANNs are capable of capturing the hidden functional relationships among the given data [177].

Neural networks consist of extensive classes of various architectures. They are composed of a number of interconnecting elements named neurons. Neural networks can 
be categorized into dynamic and static networks. Static or feedforward networks do not have any delay or feedback elements. On the contrary, the output of dynamic networks depends on the current, previous inputs or outputs of the network [178]. In this section, a feedforward neural network and two dynamic neural networks, the Elman neural network and the NARX neural network, are reviewed.

\subsubsection{Feedforward Neural Network}

The feedforward neural network is the most popular and most widely used architecture and consists of an input layer, several hidden layers, an output layer, a summation and a nonlinear transfer function as shown in Figure 3.1.

\section{Input Layer Hidden Layer Output Layer}

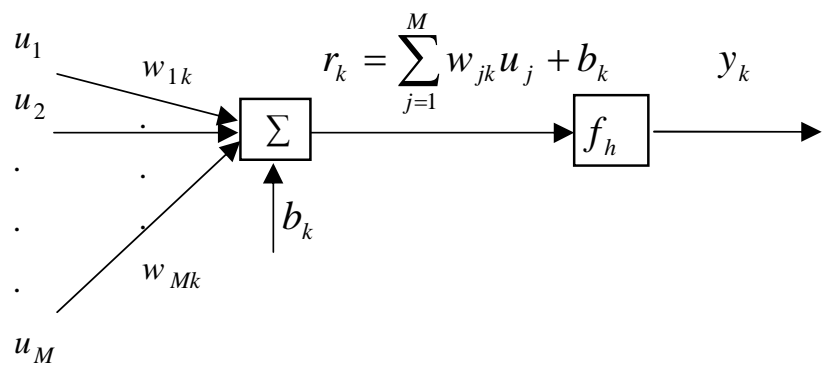

Figure 3.1 Single node in a feedforward neural network.

The inputs $u_{j}, j=1,2, \ldots, M$ to the input layer are multiplied by the weights $w_{j k}$, where $k$ represents the number of neurons in the hidden layer, and added up with the bias $b_{k}$. The obtained value $r_{k}$ is the input to the transfer function $f$ [179]. The output of node $k$ is calculated as shown in Eq. (15).

$$
y_{k}=f\left(\sum_{j=1}^{M} w_{j k} u_{j}+b_{k}\right)
$$


A multi-layer feedforward (MLFF) network is structured by connecting several nodes. A typical feedforward neural network is shown in Figure 3.2.

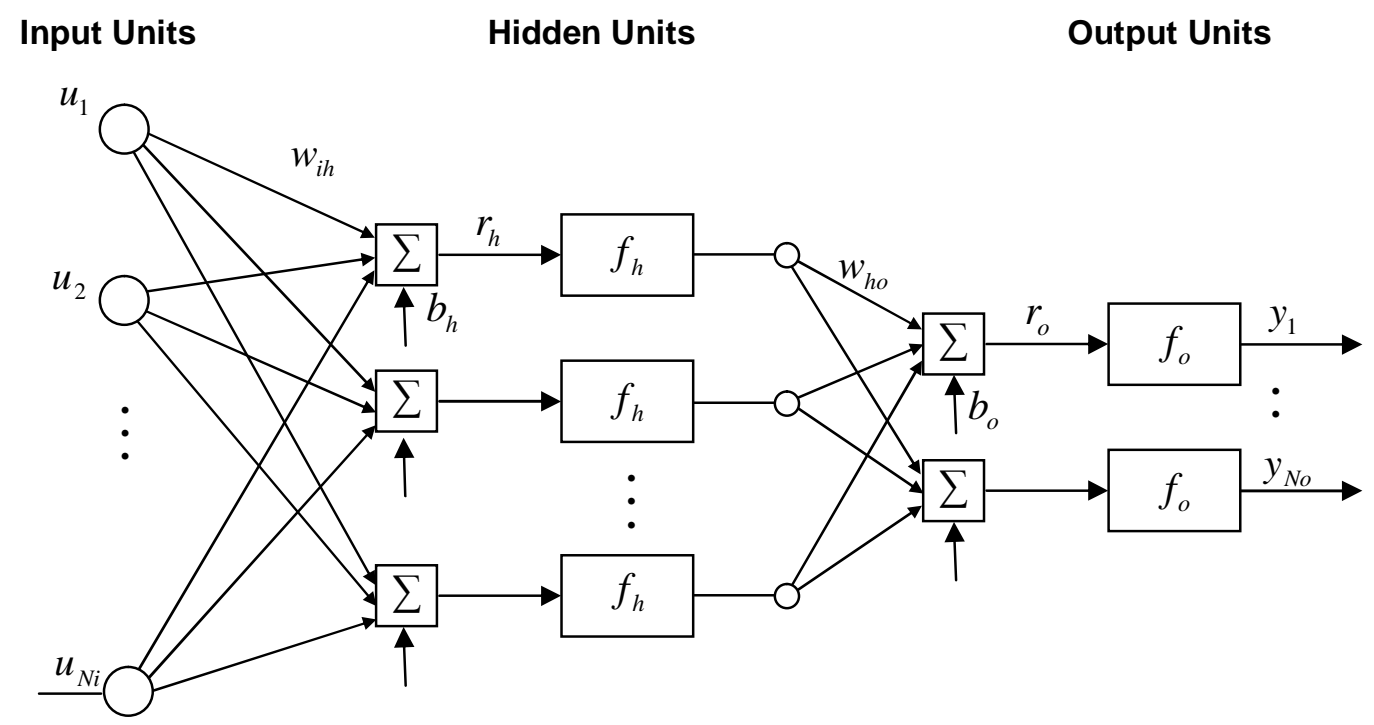

Figure 3.2 The architecture of feedforward neural network.

Given input data $u_{i}, i=1,2, \ldots, N i$ and output data $y_{k}, k=1,2, \ldots, N o$ finding the best feedforward network can be formulated as a least squares fit by Eq. (16).

$$
y_{k}=f_{o}\left[\sum_{o=1}^{N o} b_{o}+\sum_{h=1}^{N h} w_{h o} \cdot f_{h}\left(r_{h}\right)\right]=f_{o}\left[\sum_{o=1}^{N o} b_{o}+\sum_{h=1}^{N h} w_{h o} \cdot f_{h}\left(b_{h}+\sum_{i=1}^{N i} w_{i h} u_{i}\right)\right]
$$

First, the parameters of the feedforward network architecture, namely, the number of hidden layers and neurons in each layer need to be selected. The inputs $u_{i}, i=1,2, \ldots, N i$ to the input neurons are multiplied by weights $w_{i h}$, with $h=1,2, . ., N h$ representing the number of neurons in the hidden layer, and summed up together with the constant bias term $b_{h}$. The resulting $r_{h}$ is the input to the transfer function $f_{h}(\bullet)$. Later, the results of the hidden layer are multiplied by weights $w_{h o}$, and summed up together with the constant bias term $b_{o}$. The 
resulting $r_{o}$ is the input to the transfer function $f_{o}(\bullet)$. Finally, the unknown parameters which are the weights, $w_{i h}$ and $w_{h o}$ and biases, $b_{h}$ and $b_{o}$ will be determined $[178,180]$.

\subsubsection{Elman Neural Network}

The Elman network belongs to the class of recurrent neural networks (RNN) architecture. The Elman network is a two-layer network and contains additional feedback connections from the output of the hidden layer to the input layer [181]. Figure 3.3 demonstrates that the Elman neural network contains four units: input, hidden, recurrent and output units. The recurrent units which transfer the previous state of the hidden units to the input layer are recognized as a one-step time delay [1].

At a certain time, the previous state of the hidden units and the present inputs are fed to the Elman network as inputs. With this arrangement, the network can be treated as a feedforward neural network and can process the inputs to generate the outputs. When the training starts, the recurrent units are receiving the feedbacks from the current state of the hidden units and the data will be kept for the next training step [1].

The primary input to the Elman network is $u_{i}, i=1,2, \ldots, N i$ and output data $y_{k}, k=$ $1,2, \ldots$, No. The architecture of the Elman network can be written mathematically by Eq. (17):

$y_{k}=f_{o}\left[\sum_{o=1}^{N o} b_{o}+\sum_{h=1}^{N h} w_{h o} \cdot f_{h}\left(b_{h}+\sum_{i=0}^{N i} w_{i h} u_{i}+\sum_{j=0}^{N h} w_{j h} a_{h}(k-1)\right)\right]$

where $w_{i h}, w_{j h}$ and $w_{h o}, i=1,2, \ldots, N i ; j, h=1,2, \ldots, N h ; o=1,2, \ldots, N o$ are the weights of the connections between the input and hidden units, between the recurrent and the hidden units, and between the hidden and the output units, respectively. $b_{h}$ and $b_{o}$ are biases of the hidden units and the output units, and $f_{h}(\bullet)$ and $f_{o}(\bullet)$ are the hidden and output 
functions, respectively [1]. The structure of Eq. (17) is similar to Eq. (16) which is generated for a feedforward neural network. However, the last term in Eq. (17), $\sum_{j=0}^{N h} w_{j h} a_{h}(k-1)$, represents the feedback from the hidden layer to the input layer

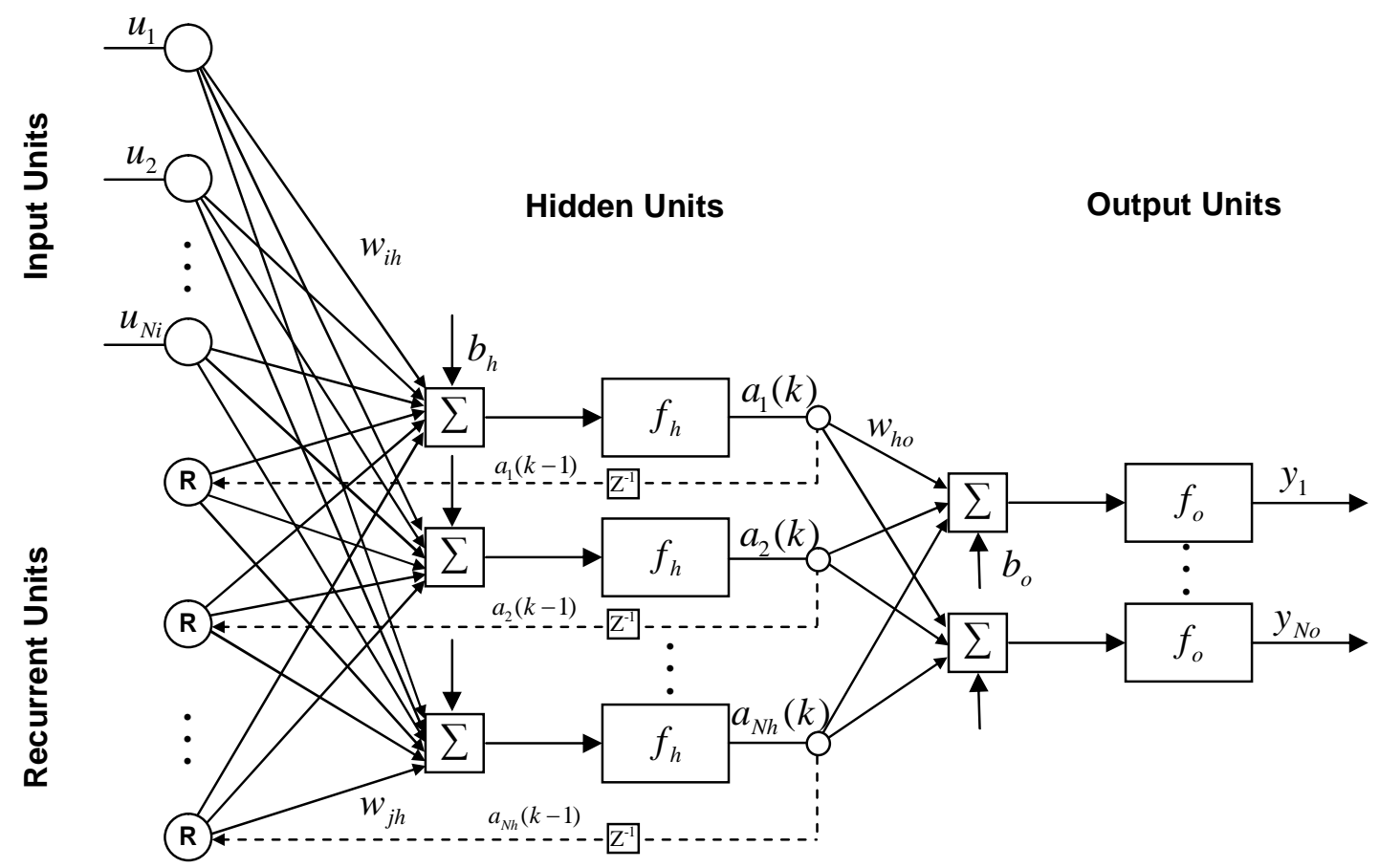

Figure 3.3 The architecture of Elman recurrent neural network.

\subsubsection{NARX Neural Network}

The nonlinear autoregressive with exogenous input (NARX) neural network is another class of ANNs which are suitable for modeling nonlinear systems and time series [15]. The NARX network is a dynamic neural network and contains recurrent feedback from several layers of the network to the input layer $[166,182,183]$. NARX can be mathematically represented by Eq. (18):

$y(n+1)=f\left[y(n), \ldots, y\left(n-d_{y}\right) ; u(n), \ldots, u\left(n-d_{u}\right)\right]$ 
where $u(n) \in \mathfrak{R}$ and $y(n) \in \mathfrak{R}$ are the input and output of the model at discrete time step $n$, respectively, and $d_{u} \geq 1$ is the input and $d_{y} \geq 1,\left(d_{y} \geq d_{u}\right)$ is the output delay. The function $f(\bullet)$ is generally unknown and can be approximated [28]. For the NARX architecture shown in Figure 3.4 with one time series as input and one time series as output, the general NARX network equation can be written as Eq. (19):

$y(n+1)=f_{o}\left[b_{o}+\sum_{h=1}^{N h} w_{h o} \cdot f_{h}\left(b_{h}+\sum_{i=0}^{d_{u}} w_{i h} u(n-i)+\sum_{j=0}^{d_{y}} w_{j h} y(n-j)\right)\right]$

where $w_{i h}, w_{j h}$ and $w_{h o}, i=1,2, \ldots, d_{u}, j=1,2, \ldots, d_{y}, h=1,2, \ldots, N h$ are the weights, $b_{h}$ and $b_{o}$ are biases and $f_{h}(\bullet)$ and $f_{o}(\bullet)$ are the hidden and output functions [28].

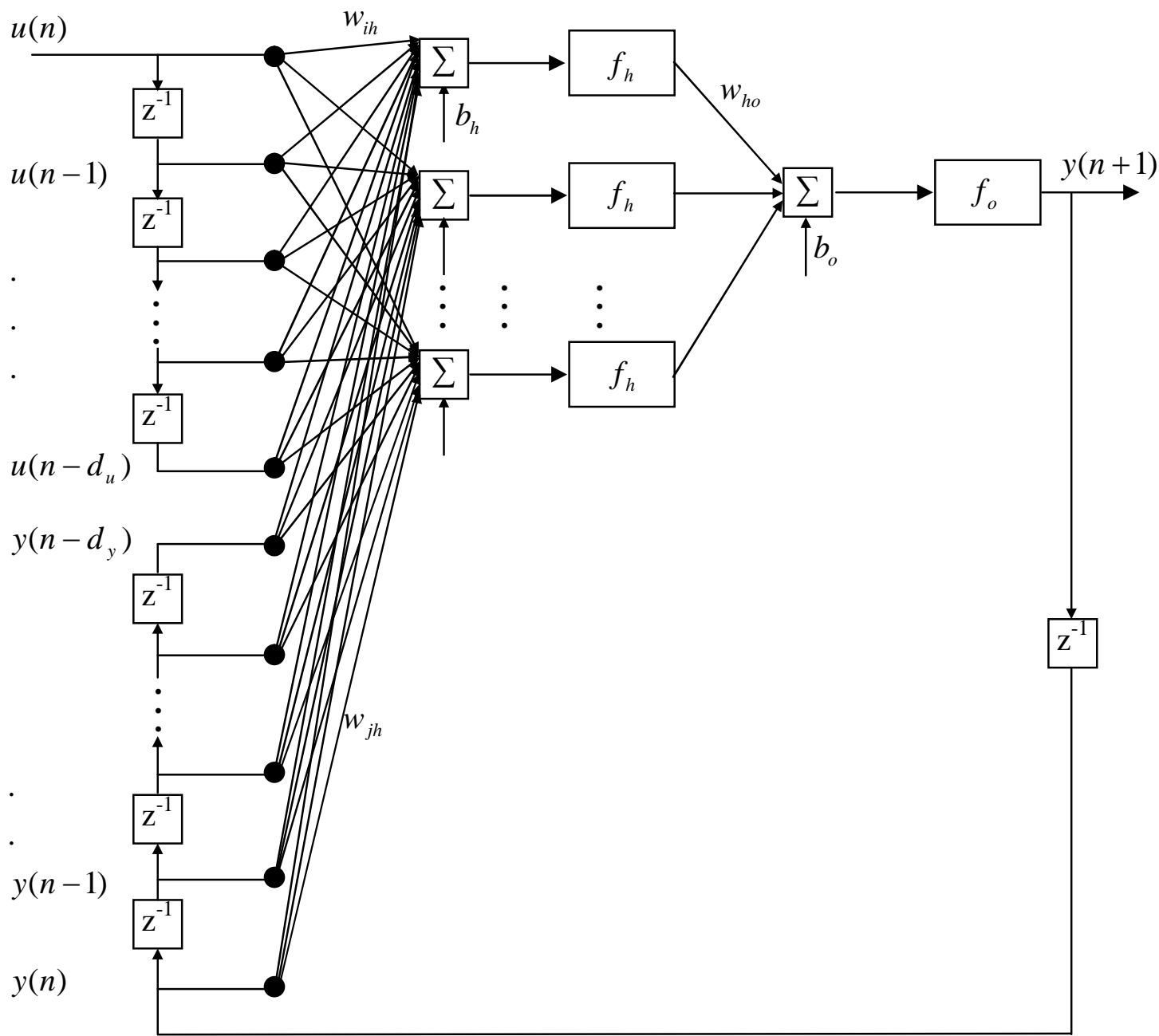

Figure 3.4 The architecture of NARX neural network. 
The NARX network can be used in multi-time series input and multi-time series output applications. The equation for a general NARX network with two inputs and one output can be written as Eq. (20):

$y(n+1)=f_{o}\left[b_{o}+\sum_{h=1}^{N} w_{h o} \cdot f_{h}\left(b_{h}+\sum_{i 1=0}^{d_{u 1}} w_{i 1 h} u_{1}(n-i 1)+\sum_{i 2=0}^{d_{u 2}} w_{i 2 h} u_{2}(n-i 2)+\sum_{j=0}^{d_{v}} w_{j h} y(n-j)\right)\right]$

where $w_{i 1 h}$ and $w_{i 2 h}, i 1=1,2, \ldots, d_{u 1}, i 2=1,2, \ldots, d_{u 2}$ are the weights of the connections between the first input units and the hidden units and between the second input units and the hidden units, respectively [28].

\subsection{Genetic Algorithms}

Genetic algorithms are an evolutionary method which simulates the process of natural selection proposed by Darwin in 1859 [184]. Genetic algorithms follow the survival strategy used by nature to modify species. In 1975, Holland [185] introduced the GA method for the first time for optimization purposes [70].

In the GA method, the optimization parameters are represented as strings of binary digits to mimic biological chromosomes. Crossover and mutation are used to generate new offspring. The format of the chromosomes is the first step in the design of the structure of a GA search. The parameters are restructured as binary strings to form strings of digits [70]. In GA literature, the space assigned to each parameter is called a gene. Each gene contains several binary digits. A chromosome is formed when several genes are combined as shown in Figure 3.5. 
Chromosome

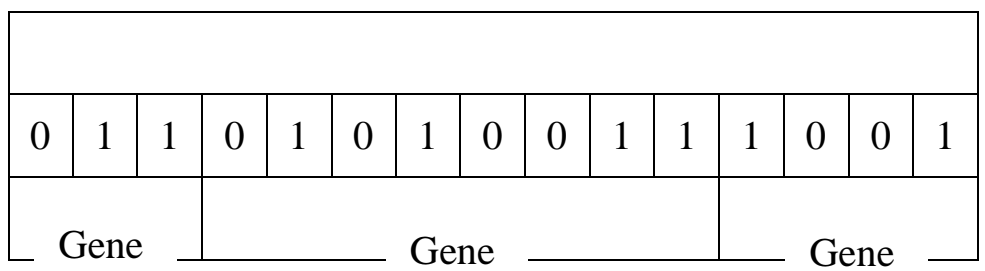

Figure 3.5 A string representing gene and chromosome in genetic algorithms.

The minimum and maximum values of the parameters are determined. The value of the genes needs to be kept between these two values. The next step is selection of population size which is the number of chromosomes for each generation. The location of crossover and the probability of mutation are required before running a GA process [70].

In the GA method, the first population of chromosomes is generated randomly. The chromosomes are broken into the genes. The values of the genes which represent the value of the corresponding parameters are tried on the overall function. The fitness of each chromosome is measured. Using Eq. (21) the probability of selecting the chromosomes for mating is determined:

$$
p_{t}=\frac{f_{t}}{\sum_{i=1}^{N} f_{i}}
$$

where $f_{t}$ represents the fitness of the chromosome, and $N$ is the population size.

Based on Eq. (21) the mating chromosomes are selected and according to the preset probability the locations of the crossovers are identified. When the crossovers take place, the mutation will apply to each bit of the chromosomes based on the preset probability. The resulting chromosomes will be transferred to the next generation and the population of the next generation will be formed as demonstrated in Figure 3.6. Over the generations, the 
best chromosome which optimizes the overall function will be selected as the best chromosome, and the value of the parameters will be selected accordingly [70].

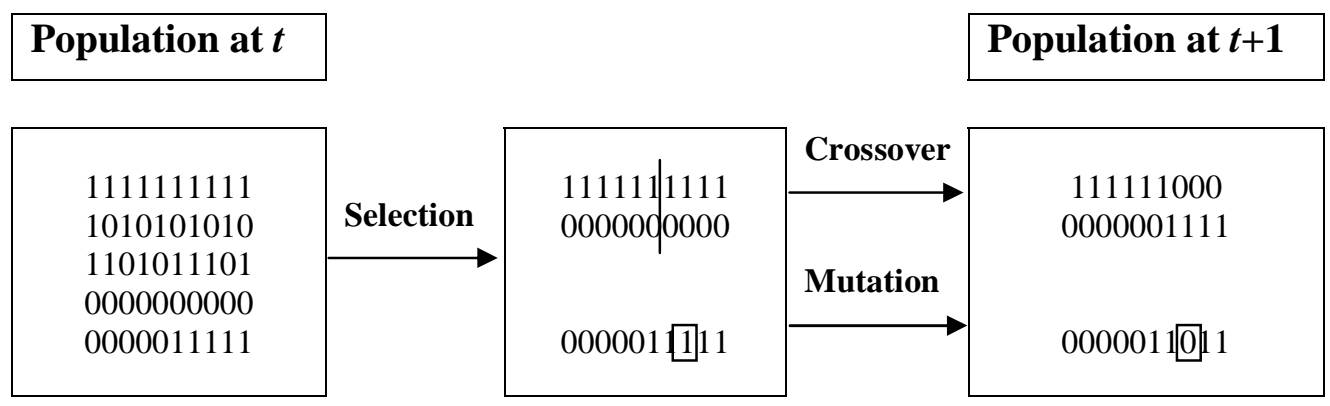

Figure 3.6 Schematic illustration of genetic algorithms.

\subsection{Summary}

The first step to analyze a system is to identify the nature of the system. Systems are classified into three main categories: stable, periodic and chaotic. Chaotic systems can be recognized by being deterministic, bounded, aperiodic, and sensitive to initial conditions, changing domains, having chaotic patterns and a chaotic attractor, invalidating the reductionism approach and underestimating computer accuracy. Chaos theory can describe complex systems mathematically. Takens' embedding theorem states that, in chaotic systems, phase space points follow the unknown dynamics of the original system. A sequence of scalar values over time which are taken in regular intervals and exhibit chaotic characteristics are called chaotic time series.

The purpose of this chapter was to introduce the tools and theories required to develop the forecasting method proposed in this dissertation. The content of this chapter is adopted in the following chapters and correlates with the block diagram in Figure 1.1. 
In Section 3.2.2, four methods, Fourier transform, Lyapunov exponent, Hurst exponent and fractal dimension, were introduced to identify the existence of chaos in a system. The application of Section 3.2.2 is reflected in the block diagram items 2 and 11 . Based on the content of Sections 3.2.3, 3.2.4 and 3.2.5, to apply the embedding theorem, proper time delay and embedding dimension are required. The application of Sections 3.2.4 and 3.2.5 is mentioned in the block diagram item 3. Also, the block diagram item 4 presents the application of Section 3.2.3.

Artificial neural networks are nonlinear models which simulate the human neural system and have the functions of self-organizing, data-driven, self-study, self-adaptive and associated memory. ANNs are capable of capturing the hidden functional relationships among the given data. The feedforward neural network is an example of static neural networks and Elman and NARX are the examples of dynamic neural networks. The content of Section 3.3 is used in the block diagram items 6 and 12. In the block diagram item 6, the artificial neural network architectures introduced in Sections 3.3.1 and 3.3.2 can be used. In the block diagram item 12, the artificial neural network architectures discussed in Sections 3.3.1 and 3.3.3 can be adopted.

Genetic algorithms as an evolutionary method which simulates the process of natural selection can be used for the purpose of optimization. Genetic algorithms in Section 3.4 can be employed to improve the result of the neural networks presented in the block diagram items 6 and 12 . 


\section{CHAPTER 4}

\section{COMBINATION OF CHAOS THEORY AND ARTIFICIAL INTELLIGENCE IN CHAOTIC TIME SERIES FORECASTING}

In this chapter, a unique technique based on chaos theory and artificial neural networks is proposed to analyze and forecast chaotic time series. The first requirement is to analyze the time series and investigate the presence of chaos in the time series. The embedding theorem is used to determine the embedding parameters $D$ and $T$, and accordingly the chaotic time series is reconstructed into the phase space points. Based on chaos theory, there exists an unknown mathematical equation which can forecast the future value of the phase space points. Artificial neural networks are employed to capture the functional relationships among the given phase space points. Therefore, the embedded phase space points are fed into a neural network and trained. The weights and biases of the trained neural network are kept to forecast the future of phase space points. When the unknown phase space points are predicted, the future values of the time series are obtained consequently. Two neural network architectures, feedforward and Elman, are utilized for forecasting. Genetic algorithms are merged with the proposed method to improve the performance of the forecasting method by selecting the best initial weights and biases for the neural networks. The Mackey-Glass, Logistic and Henon time series are used to validate the performance of the proposed technique. The numerical experimental results 
confirm that the proposed method can forecast the chaotic time series effectively and accurately when compared with the existing forecasting methods.

The content of the current chapter has been presented at the Canadian Operational Research Society (CORS) conference [186], published in the Proceedings of the 3rd World Conference on Production and Operations Management [187] and accepted for publication by the International Journal of Applied Management Science [188].

The current chapter is structured as follows. In Section 4.1, a novel forecasting method developed in this research work, which is based on the precise definition of chaos theory, is presented. In order to evaluate the performance of the proposed method, three well-known chaotic time series, Mackey-Glass, Logistic and Henon are used for benchmarking. In Section 4.2, feedforward neural network architecture is selected to forecast the chaotic time series. In Section 4.3, Elman RNN architecture is selected to forecast the chaotic time series. In Section 4.4, genetic algorithms are utilized to improve the performance of the neural networks. Section 4.5 compares the performance of the proposed forecasting method in this chapter with the results presented in similar research works in the literature. Section 4.6 concludes Chapter 4.

\subsection{Introducing the Proposed Forecasting Method}

This section introduces an original technique which has been developed to forecast one step ahead of a given chaotic time series. The current state is used to forecast the next state. When the next state is predicted, the real value of the next state is used to forecast the following state and so on. The key difference between this technique and the techniques proposed in the literature $[1,13]$ is the utilization of the precise definition of chaos theory and the embedding theorem. 
Chaos theory states that for a chaotic system, there exists an unknown mathematical equation which can describe the system, Eq. (2). On the other hand, Taken's [20] embedding theorem claims that in phase space mode, a chaotic system reflects most of the characteristics of the original system. Therefore, $s(t)$ in Eq. (2) can be replaced by $Y(t)$. Also, unknown function $Q: R^{n} \rightarrow R^{n}$ can be estimated by function $G$ : $R^{D} \rightarrow R^{D}$ where $D$ is the embedding dimension. $D$ mimics $n$, the dimension of the original state. Eq. (2) at time $t+1$ can be rewritten as Eq. (22) which is the next state of the system. $Y(t) \rightarrow G(Y(t))=Y(t+1)$

The contribution of ANN is to find an appropriate relationship between the current state of the system and the next state of the system. In the current technique, an artificial neural network is trained using a single phase space point (current state) as input and a single phase space point (next state) as output. This complies precisely with the definition of chaos theory and the embedding theorem.

On the other hand, in the forecasting methods proposed in the literature an artificial neural network is trained either using a single phase space point as input and a single point from the time series as output [13] or with multiple phase space points as input and a single phase space point as output [1]. None of these approaches makes full use of the precise definition of chaos theory.

Figure 4.1, which is Block $\mathrm{A}$ in Figure 1.1 demonstrates the required steps to develop the proposed forecasting method. Based on the observed time series data set, $x(1), x(2), x(3), \ldots, x(N)$, where $N$ is the length of the data set, the presence of chaos is examined by Fourier transform, Lyapunov exponent, Hurst exponent and fractal dimension as mentioned in Section 3.2.2. When the presence of chaos is detected in the 
observed time series then the phase space parameters $D$ and $T$ are calculated as described in Sections 3.2.4 and 3.2.5. Based on the discussion in Section 3.2.3, $[N-(D-1) T]$ phase space points $Y(j), j=1 \sim[N-(D-1) T]$, are generated as shown in Eq. (23).

$Y(1)=[x(1), x(1+T), \ldots, x(1+(D-1) T)]$

$Y(2)=[x(2), x(2+T), \ldots, x(2+(D-1) T)]$

$Y(j)=[x(N-(D-1) T), x(N-(D-1) T+T), \ldots, x(N)]$

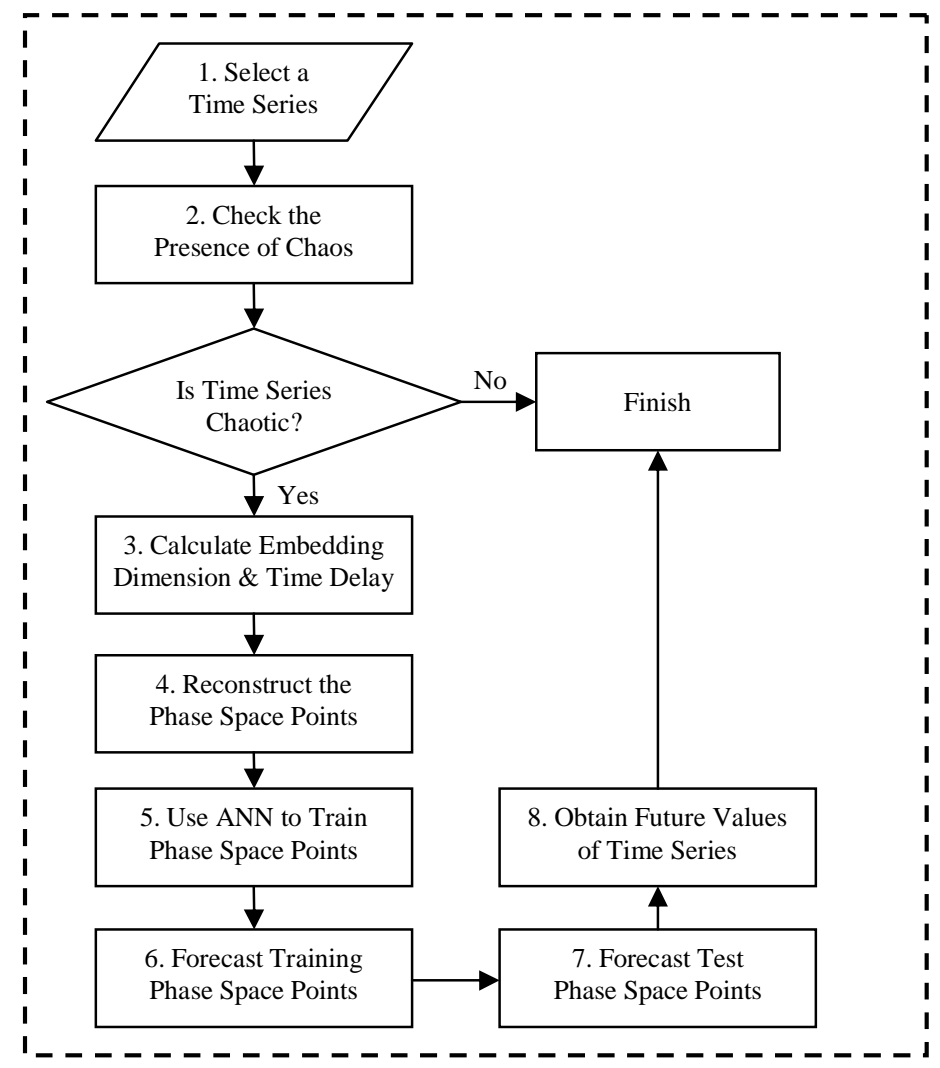

Figure 4.1 A block diagram demonstrating the proposed forecasting method.

An artificial neural network with a single phase space point, $D$ input units, and a single phase space point, $D$ output units, is trained. The generated phase space points as 
inputs, $Y(i), i=1 \sim[N-(D-1) T-1]$ and outputs $Y(k), k=2 \sim[N-(D-1) T]$ are denoted by Eq. (24) and Eq. (25), respectively.

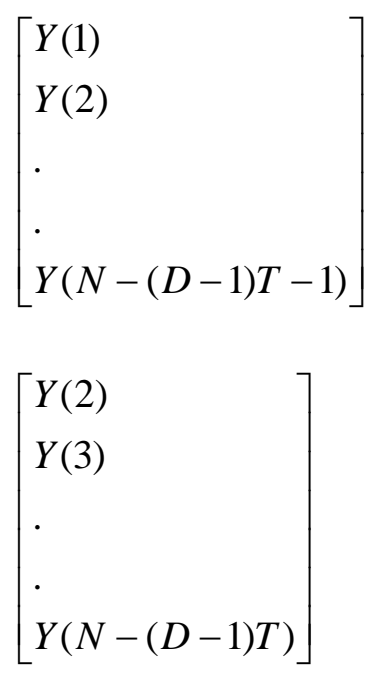

After training the artificial neural network, the weights and biases of the network are kept to forecast the unknown phase space points. When the neural network training is completed, based on the known vector, $Y(N-(D-1) T)$ as input to the trained neural network, the unknown vector, $Y(N-(D-1) T+1)$ is determined. $Y(N-(D-1) T+1)$ is composed of known values $x(N-(D-1) T+1), x(N-(D-1) T+1+T), \ldots, \quad x(N-(D-1) T+1+(D-2) T)$ and unknown value $x(N+1)$. When $Y(N-(D-1) T+1)$ is determined, the only unknown value, $x(N+1)$, can be obtained.

\subsection{Numerical Analysis Using Feedforward Neural Network}

In order to evaluate the performance of the proposed method, the developed technique is applied to three chaotic time series: Mackey-Glass, Logistic and Henon. In this section, the feedforward neural network is used to estimate the unknown chaotic function. 


\subsubsection{Mackey-Glass Equation}

The Mackey-Glass equation originally was proposed as a model of blood cell regulation [189]. The Mackey-Glass time series has been used in the literature as a benchmark model due to its chaotic characteristics. The differential equation leading to the time series is demonstrated in Eq. (26).

$\frac{d x}{d t}=\frac{a x(t-\tau)}{\left[1+x^{c}(t-\tau)\right]}-b x(t)$

In the Mackey-Glass equation the delay parameter, $\tau$, determines the nature of the chaotic behaviour of the Eq. (26); i.e. $\tau<4.43$ produces a fixed point attractor, 4.43 $<\tau<13.3$ a stable limit cycle attractor, $13.3<\tau<16.8$ a double limit cycle attractor and $\tau>16.8$ chaos. In order to compare the results of the proposed technique with the results published in the literature, the parameters are selected according to the previous report [1], where the constants are taken to be $a=0.2, b=0.1$ and $c=10$, and chaotic time series is generated by $\tau=17$ and initial value $x(0)=1.2$.

A chaotic data set with 1000 data points is generated by Eq. (26) and reconstructed into the phase space points. The first 500 data points are used for training and the remaining 500 data points are kept to test the performance of the proposed method. The embedding dimension $D$ and the time delay $T$ are calculated using TSTOOL [190], an addon toolbox for MATLAB [191]. The time series are reconstructed with $D=3$ and $T=7$. The data in the time domain and phase space domain are shown in Figure 4.2.

The embedded phase space points $Y(t)$ are generated based on the first 500 data points. A feedforward neural network is trained using the reconstructed phase space points. The number of the hidden layer nodes is selected via trial-and-error, which is the most common way in the literature. The weights and biases of the neural networks are kept to 
forecast the unknown phase space points. Finally, the forecasted phase points are decomposed to forecast the future values of the time series as shown in Figure 4.3.

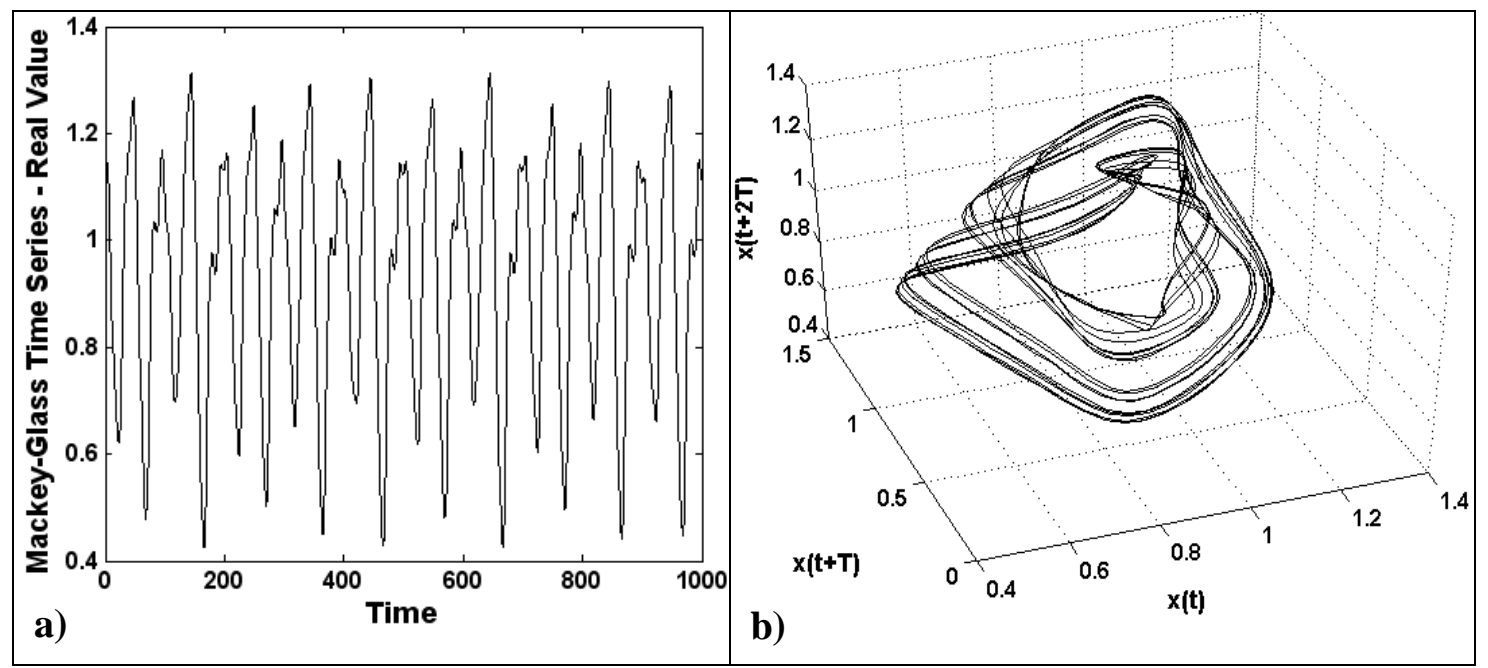

Figure 4.2 Mackey-Glass chaotic time series a) in time domain, b) in phase space domain.

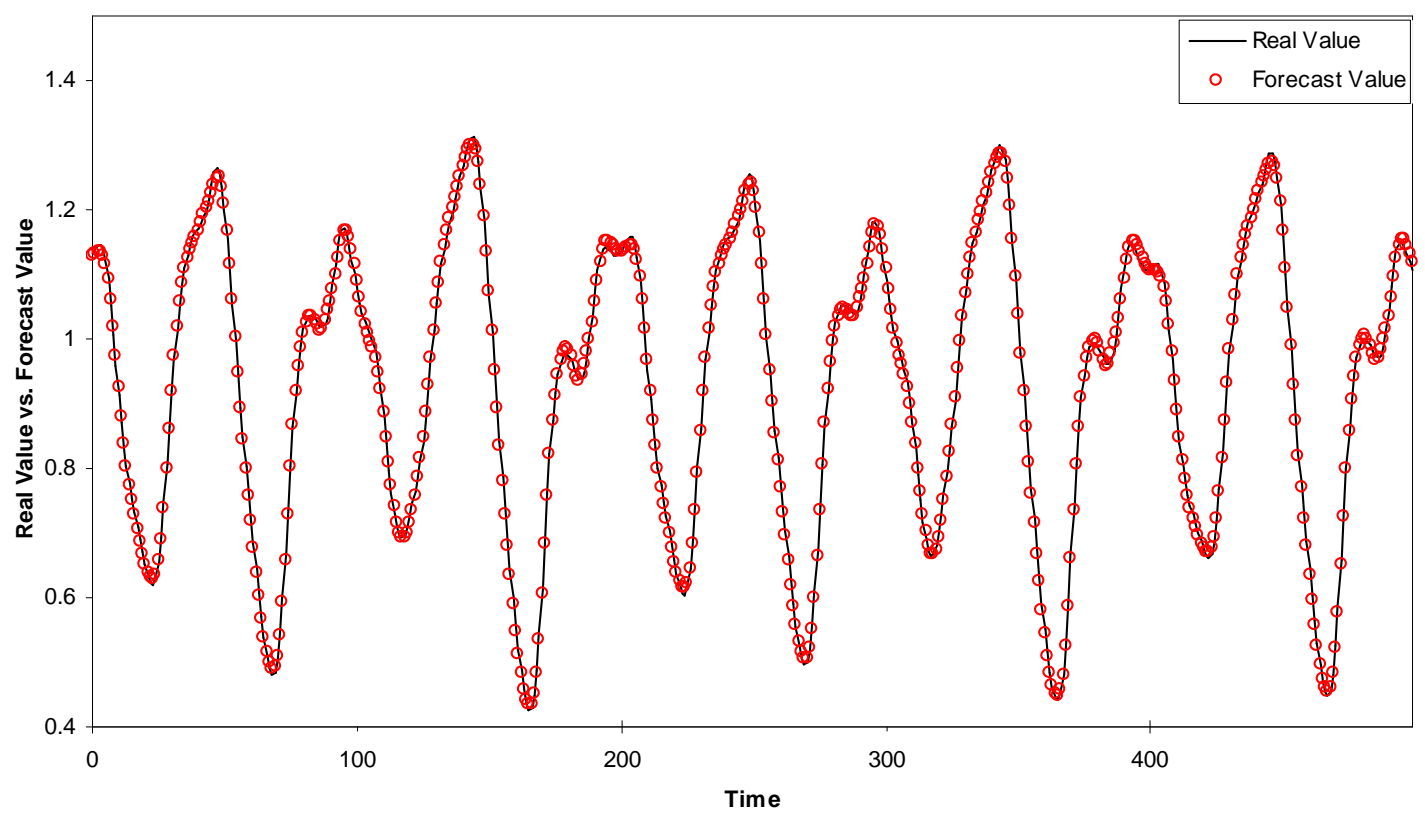

Figure 4.3 Comparison of Mackey-Glass time series and forecast values using FFNN. 
Based on the selected research works for comparison, the error indices are calculated. In order to evaluate the prediction performance and compare it with the results reported in the literature, the mean squared error (MSE) and the normalized mean squared error (NMSE) are calculated by Eq. (27) and Eq. (28), respectively.

$$
\begin{aligned}
& M S E=\frac{1}{N} \sum_{i=1}^{N}\left(y_{i}-\hat{y}_{i}\right)^{2} \\
& N M S E=\left(\frac{\sum_{i=1}^{N}\left(y_{i}-\hat{y}_{i}\right)^{2}}{\sum_{i=1}^{N}\left(y_{i}-\bar{y}\right)^{2}}\right)
\end{aligned}
$$

where $y_{i}, \hat{y}_{i}$ and $\bar{y}$ are observed data, predicted data and the average of observed data, respectively, and $N$ is the length of observed data. The MSE and NMSE values are reflected in Table 4.1. Appendix D exhibits the regression analysis between the network response and the corresponding targets for the feedforward neural network.

Table 4.1 Mackey-Glass time series - The MSE and NMSE for the 500 test samples.

\begin{tabular}{llll}
\hline & \multicolumn{2}{c}{ Prediction Error } \\
\hline Prediction Method & Reference & MSE & NMSE \\
\hline Proposed Method - FFNN & & $5.98 \mathrm{E}-05$ & $1.20 \mathrm{E}-03$ \\
\hline
\end{tabular}

\subsubsection{Logistic Equation}

The Logistic equation is the most famous, oldest and simplest chaotic mathematical system $[70,171,192]$. Although it has only one control parameter and one variable, it demonstrates chaotic behaviour. The Logistic equation had been used in insect studies [193] and fish population growth [194], however, physicist and mathematical biologist May [195] revealed the importance of the Logistic equation [171] as a chaotic system. The Logistic equation is written as Eq. (29). 
$x(t+1)=r x(t)[1-x(t)]$

With an initial state of $x(0)=0.36$ and $r=4.0$, a chaotic time series with 612 data points is generated using Eq. (29). The data in the time domain is shown in Figure 4.4. The embedding dimension $D=3$ and the time delay $T=1$ are selected based on Zhang et al. [196] and the time series is reconstructed.

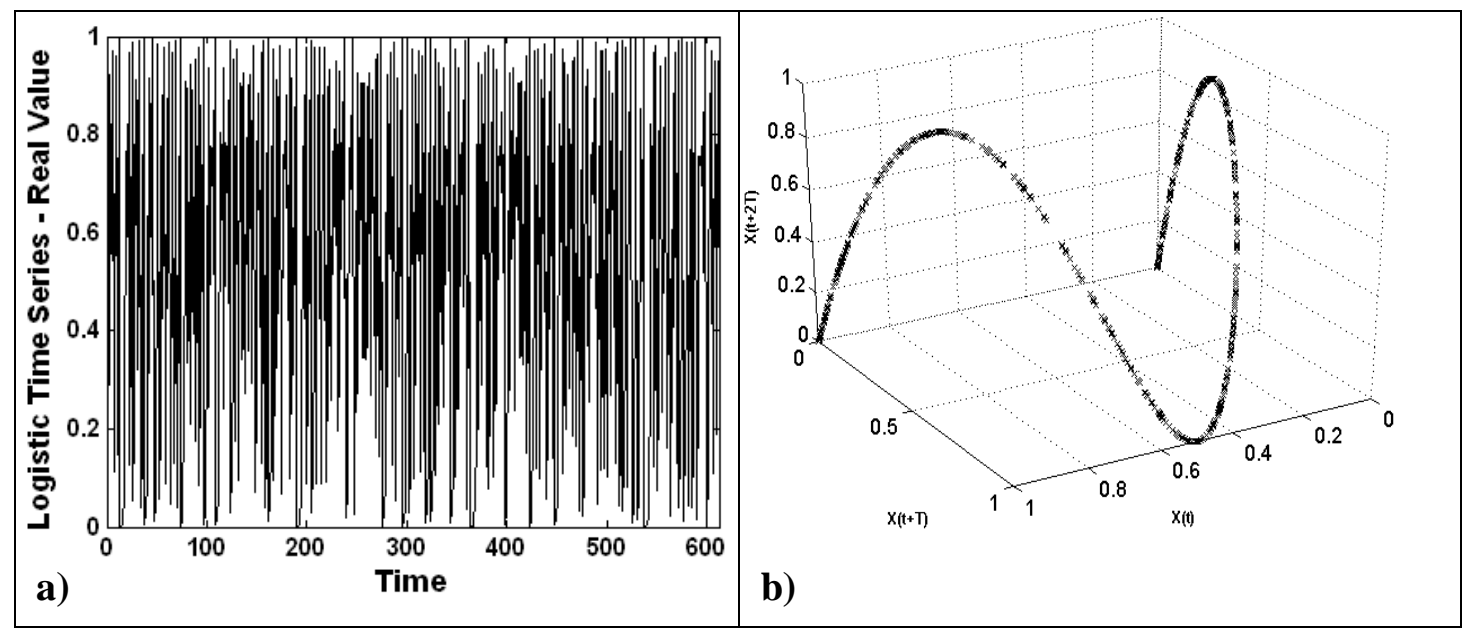

Figure 4.4 Logistic chaotic time series a) in time domain, b) in phase space domain.

The embedded phase space points $Y(t)$ are obtained based on the first 512 data points. The feedforward neural network is trained using the phase space points. The remaining 100 data points are kept to examine the performance of the proposed forecasting method. The weights and biases are kept to forecast the unknown phase space points and consequently the future values of the time series as shown in Figure 4.5.

The MSE and NMSE are calculated to evaluate the performance of the proposed method when applied to the Logistic time series and shown in Table 4.2. Appendix D exhibits the regression analysis between the network response and the corresponding targets for feedforward neural networks. 


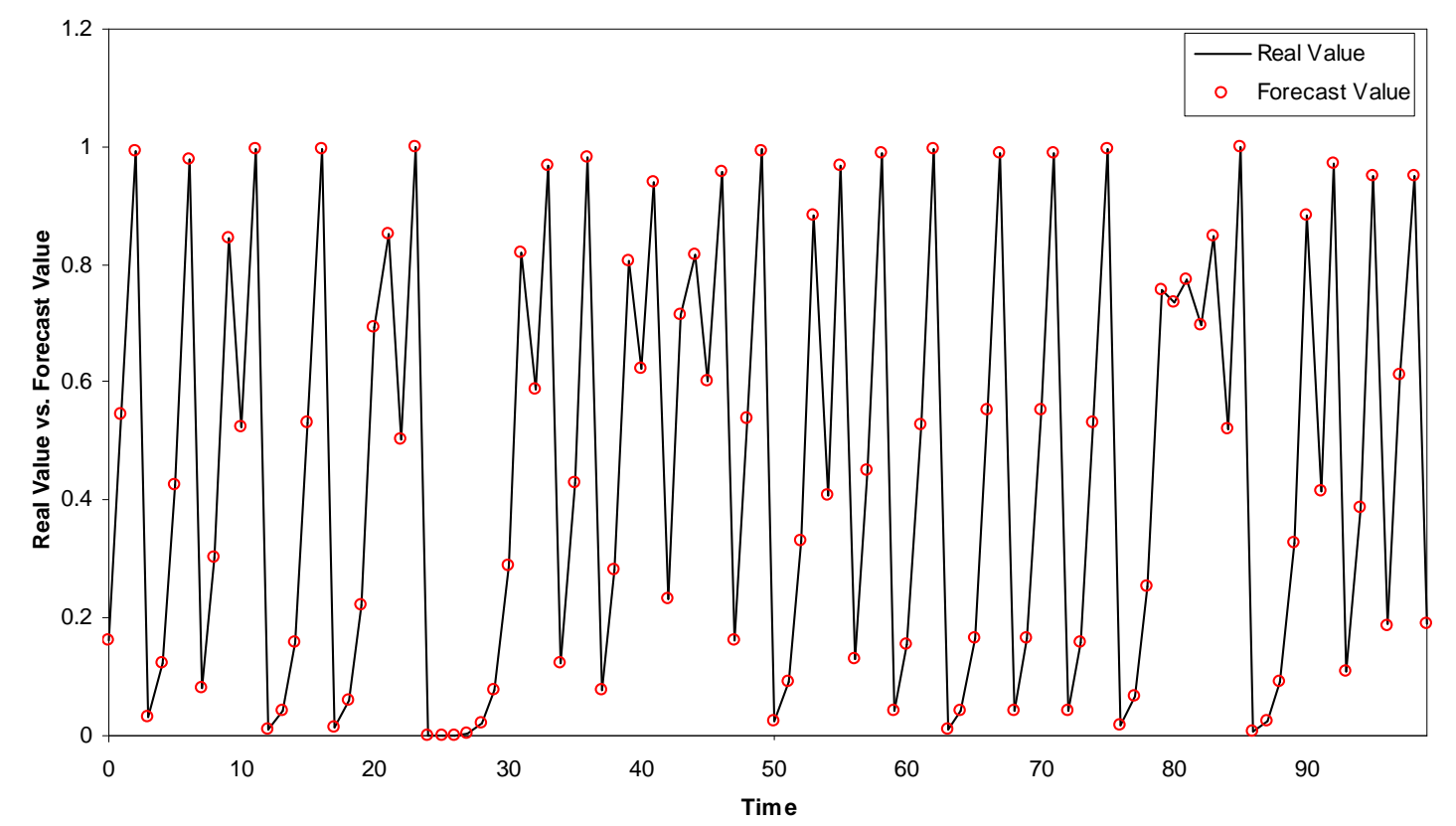

Figure 4.5 Comparison of Logistic time series data and forecast values using FFNN.

Table 4.2 Logistic time series - The MSE and NMSE for the 100 test data points.

\begin{tabular}{llll}
\hline & \multicolumn{2}{c}{ Prediction Error } \\
\hline Prediction Method & Reference & MSE & NMSE \\
\hline Proposed Method - FFNN & & $4.00 \mathrm{E}-07$ & $3.00 \mathrm{E}-06$ \\
\hline
\end{tabular}

\subsubsection{Henon Equation}

The Henon equation was first introduced by Henon [197] in 1976. The Henon equation has a simple format; however, it presents many aspects of dynamical behaviour of more complicated chaotic systems [198]. Henon equation is described by Eq. (30), which generates chaotic time series.

$x_{t+1}=B x_{t-1}+1-A x_{t}^{2}$ 
In order to compare the results of the proposed technique with the results published in the literature, the parameters are selected according to Fanzi and Zhengding [156], where the constants are taken to be $A=1.4, B=0.3, x_{0}=0.3$ and $x_{1}=0.3$.

A chaotic time series with 1000 samples is generated by Eq. (30). The first 500 data points are used for training and the remaining 500 points are kept for testing. The embedding dimension $D$ and the time delay $T$ are calculated using TSTOOL [190]. The time series are reconstructed with $D=2, T=1$. The data in the time domain and the phase space domain are shown in Figure 4.6.

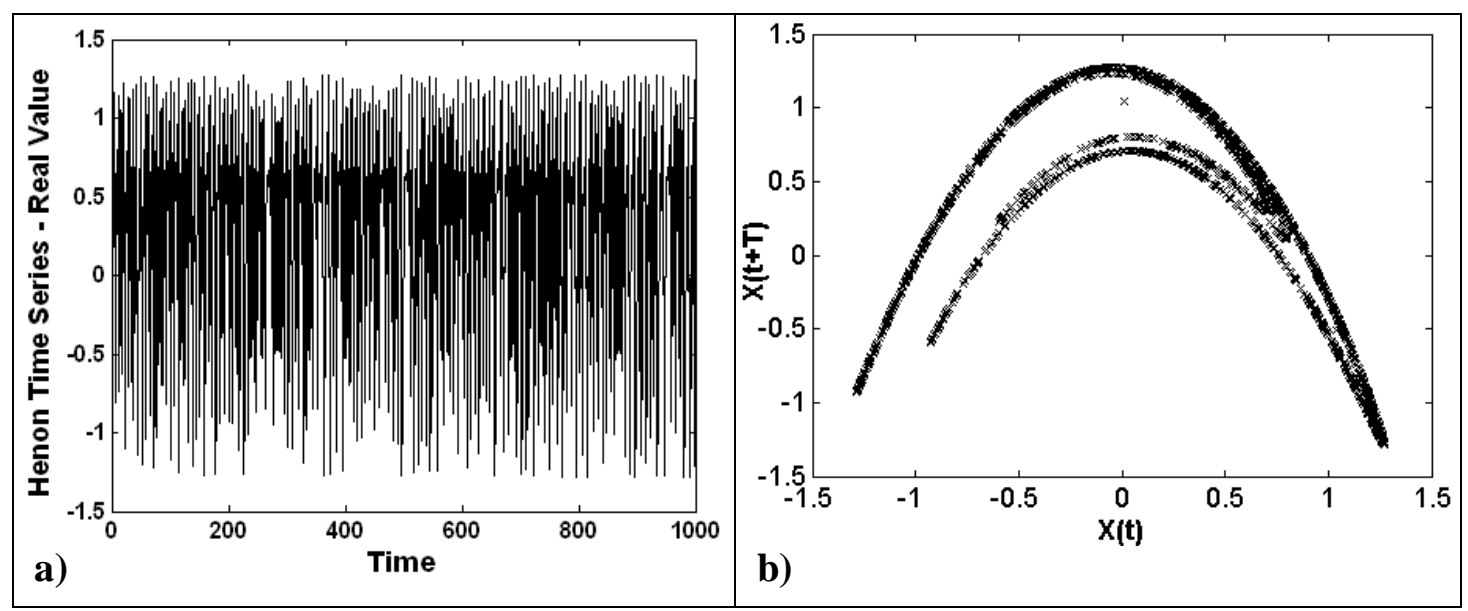

Figure 4.6 Henon chaotic time series a) in time domain, b) in phase space domain.

The embedded phase space points $Y(t)$ are generated based on the first 500 data points. The remaining 500 data points are kept for testing. The feedforward neural network architecture is selected and trained. The number of the hidden layer nodes is selected via trial-and-error which is the most common way in the literature. The weights and biases of the neural networks are kept to forecast the unknown phase space points. Finally, the forecasted phase points are decomposed to forecast the future values of the time series as shown in Figure 4.7. 


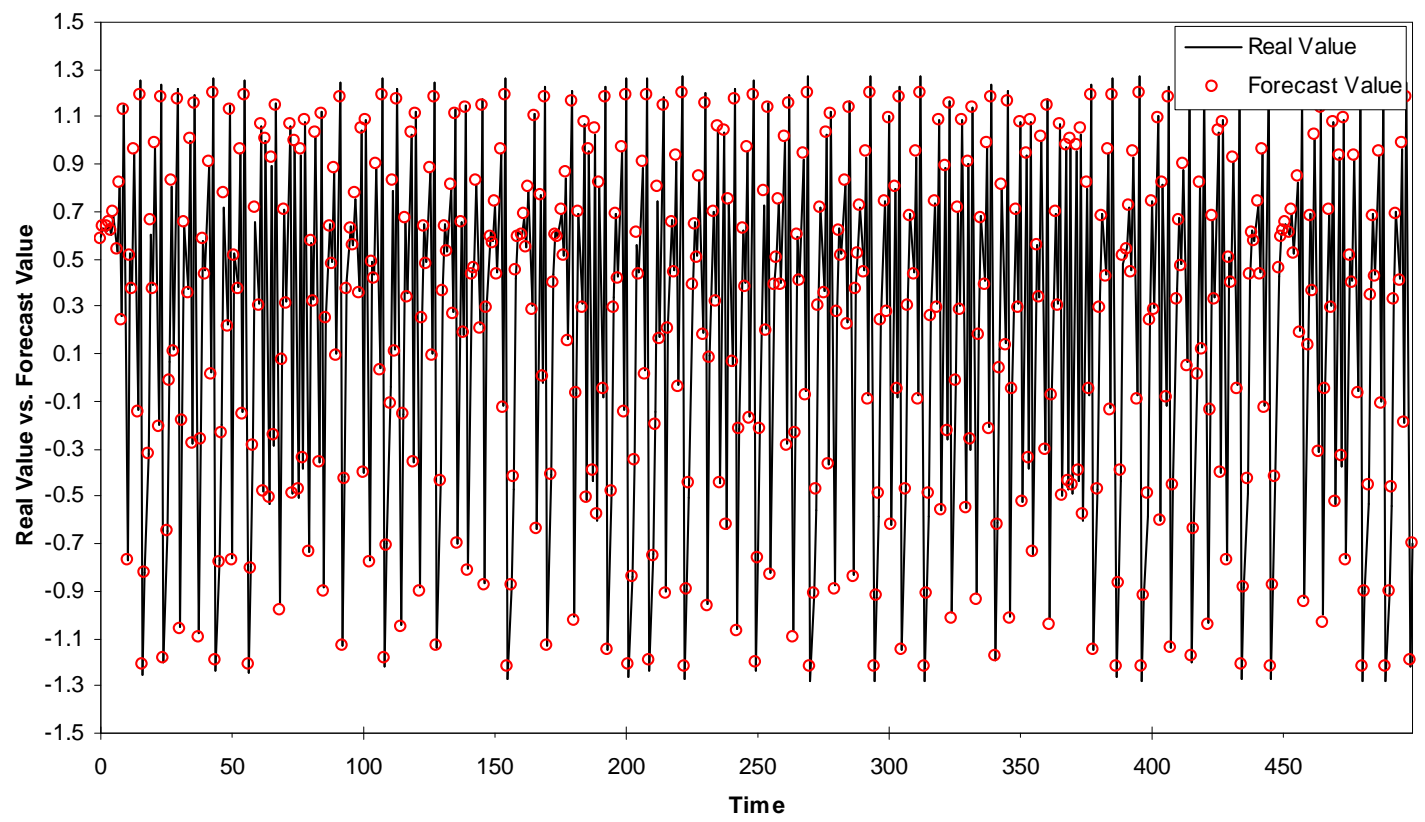

Figure 4.7 Comparison of Henon time series data and forecast values using FFNN.

In order to evaluate the performance of the proposed method, the mean square error (MSE) and normalized mean square error (NMSE) for the predicted time series values are calculated. The obtained MSE and NMSE are compared with the reported studies in the literature and reflected in Table 4.3. The results show that the proposed method can forecast the Henon time series effectively. Appendix D exhibits the regression analysis between the network response and the corresponding targets for feedforward neural networks.

Table 4.3 Henon time series - The MSE and NMSE for the 500 test data points.

\begin{tabular}{llll}
\hline & \multicolumn{2}{c}{ Prediction Error } \\
\hline Prediction Method & Reference & MSE & NMSE \\
\hline Proposed Method - FFNN & & $8.51 \mathrm{E}-04$ & $1.6 \mathrm{E}-03$ \\
\hline
\end{tabular}




\subsection{Numerical Analysis Using Elman Neural Network}

The experiments in Section 4.2 which are performed with a feedforward neural network are repeated with an Elman recurrent neural network. The conditions are kept the same to be able to compare the results. The only difference between the Elman recurrent network and the feedforward neural network is the feedback mechanism which transfers the information in hidden layer to the input layer. Figure 4.8, Figure 4.9 and Figure 4.10 exhibit the outcomes of the proposed forecasting method when applied to the MackeyGlass (M-G), Logistic and Henon time series using the Elman recurrent network.

Table 4.4 presents the performance of the proposed forecasting method using the Elman recurrent network for the three chaotic time series: Mackey-Glass, Logistic and Henon. Appendix D exhibits the regression analysis between the network response and the corresponding targets for Elman neural networks.

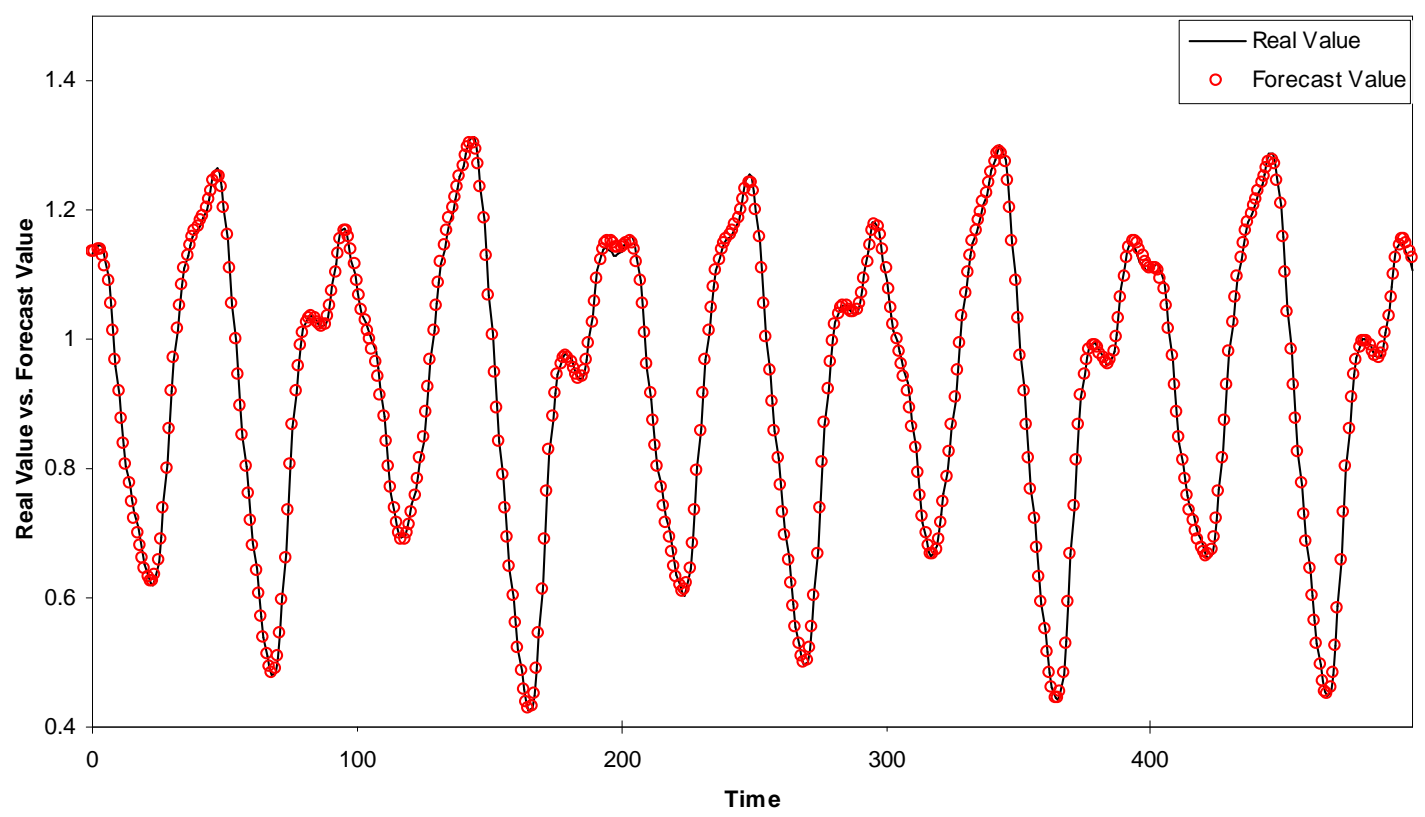

Figure 4.8 Comparison of M-G time series data and forecast values using Elman ANN. 


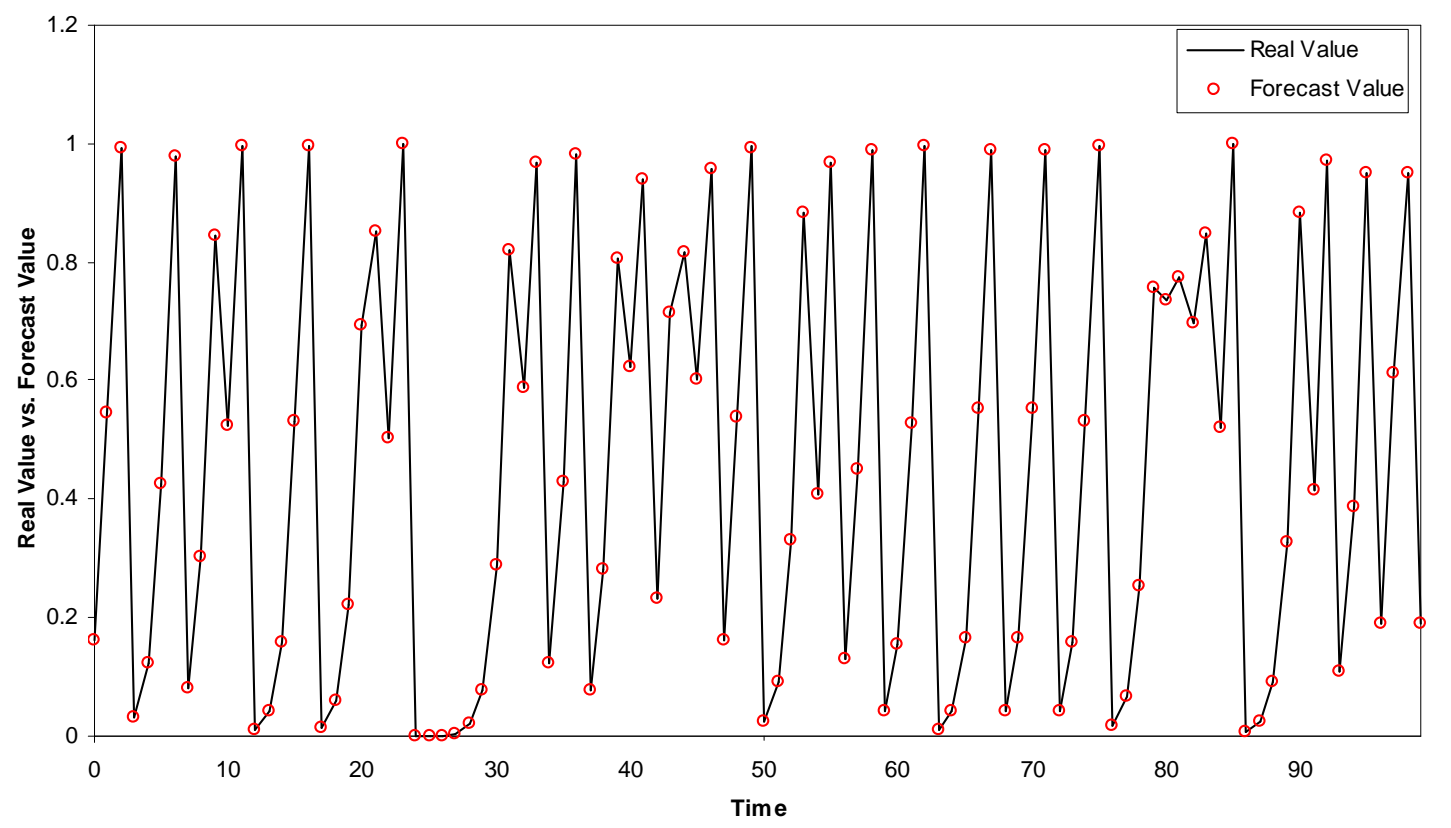

Figure 4.9 Comparison of Logistic time series data and forecast values using Elman RNN.

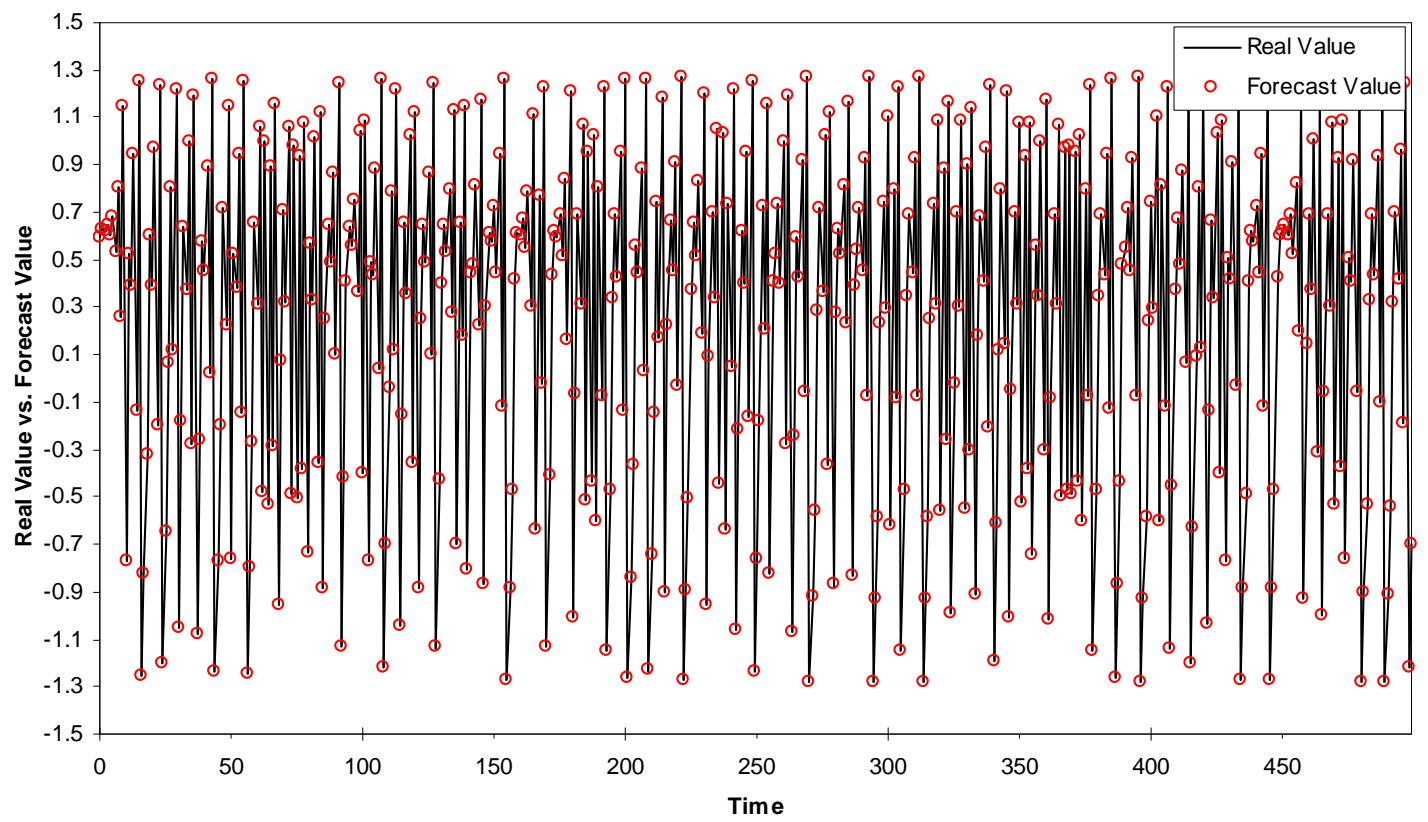

Figure 4.10 Comparison of Henon time series data and forecast values using Elman RNN. 
Table 4.4 The prediction performance using Elman RNN.

\begin{tabular}{lll}
\hline & \multicolumn{2}{c}{ Prediction Error } \\
\hline Time Series & $M S E$ & NMSE \\
\hline Mackey-Glass & $4.12 \mathrm{E}-05$ & $8.05 \mathrm{E}-04$ \\
Logistic & $2.44 \mathrm{E}-11$ & $1.89 \mathrm{E}-10$ \\
Henon & $5.41 \mathrm{E}-09$ & $1.01 \mathrm{E}-08$ \\
\hline
\end{tabular}

\subsection{Improving the Results Using Genetic Algorithms}

In most training algorithms, the initial weights and biases of the neural network are selected randomly. This will cause variation in the output of the neural network. To reduce the variation of the neural network output and improve the performance of the prediction, genetic algorithms (GA) are adopted to find appropriate initial weights and biases. The GA objective function is to minimize the mean square error (MSE) between the predicted values and the original values. The GA variables are weights and biases of the neural network. When the GA is applied, the best weights and biases which improve MSE are selected.

Table 4.5 presents the performance of the proposed forecasting method improved by genetic algorithms for both feedforward and Elman recurrent neural networks when applied to the three chaotic time series: Mackey-Glass, Logistic and Henon.

Table 4.5 The prediction performance improved by genetic algorithms.

\begin{tabular}{lll}
\hline & \multicolumn{2}{c}{ Prediction Error } \\
\hline Time Series & $M S E$ & NMSE \\
\hline Mackey-Glass - Feedforward & $5.24 \mathrm{E}-05$ & $1.0 \mathrm{E}-03$ \\
Mackey-Glass - Elman & $3.67 \mathrm{E}-05$ & $7.16 \mathrm{E}-04$ \\
Logistic - Feedforward & $2.54 \mathrm{E}-08$ & $1.90 \mathrm{E}-07$ \\
Logistic - Elman & $1.62 \mathrm{E}-11$ & $1.21 \mathrm{E}-10$ \\
Henon - Feedforward & $6.53 \mathrm{E}-04$ & $1.20 \mathrm{E}-03$ \\
Henon - Elman & $4.76 \mathrm{E}-09$ & $9.01 \mathrm{E}-09$ \\
\hline
\end{tabular}




\subsection{Comparing the Results}

The results obtained in this chapter based on the proposed method are compared with the results presented in the literature. For the Mackey-Glass time series, the performance of the proposed method in this chapter is compared with the results of a simple feedforward neural network and the methods delivered by Zhang and Man [1] and Zhang et al. [39]. The method suggested by Zhang et al. [39] utilizes the application of the delay coordinate embedding technique in the multi-input multi-output adaptive-networkbased fuzzy inference system (MANFIS) to forecast chaotic time series. Zhang and Man [1] used an Elman recurrent neural network to forecast chaotic time series. To improve their results, Zhang and Man [1] proposed a multi-dimensional method which combines the output of several trained neural networks with different embedding dimensions.

Table 4.6 compares the performance of the proposed method with the results presented in the literature. Based on this comparison, the proposed method using an Elman recurrent neural network and improved by genetic algorithms shows better performance.

Table 4.6 Mackey-Glass time series - comparing the prediction performance.

\begin{tabular}{llll}
\hline & & \multicolumn{2}{c}{ Prediction Error } \\
\hline Prediction Method & Reference & $M S E$ & NMSE \\
\hline Neuro-Fuzzy & {$[39]$} & & $1.26 \mathrm{E}-03$ \\
RNN $-D=2$ & {$[1]$} & $1.02 \mathrm{E}-03$ & \\
RNN - D 3 & {$[1]$} & $4.15 \mathrm{E}-04$ & \\
RNN - Multi-dimension & {$[1]$} & $3.81 \mathrm{E}-04$ & \\
Simple Feedforward & & $9.75 \mathrm{E}-04$ & $1.9 \mathrm{E}-02$ \\
Proposed method - Elman & & $4.12 \mathrm{E}-05$ & $8.05 \mathrm{E}-04$ \\
Proposed method - Elman \& GA & & $3.67 \mathrm{E}-05$ & $7.16 \mathrm{E}-04$ \\
\hline
\end{tabular}


For the Logistic time series, the performance of the proposed method is compared with the results of a simple feedforward neural network and the results presented in the research works by Zhang et al. [196] and Ma et al. [27]. Zhang et al. [196] developed a forecasting method based on the fundamental characteristics of chaotic systems which is sensitivity to the initial conditions. The Lyapunov exponent delivers a quantitative measurement on the dynamics of a chaotic system in various embedding dimensions. In this method, Zhang et al. [196] has used a Lyapunov exponent model to predict the unknown phase space points and consequently unknown values in the time series.

Ma et al. [27] proposed a forecasting method based on evolving recurrent neural networks (ERNN), which estimates the proper embedding parameters to reconstruct a chaotic time series and improves the structure of RNN by evolutionary algorithms.

Table 4.7 compares the performance of the proposed forecasting method in this study when applied to the Logistic time series with the results presented in the literature. The combination of the Elman RNN and GA method shows the best result in Table 7.

Table 4.7 Logistic time series - comparing the prediction performance.

\begin{tabular}{llll}
\hline & & \multicolumn{2}{c}{ Prediction Error } \\
\hline Prediction Method & Reference & $M S E$ & NMSE \\
\hline Lyapunov Method $-D=2$ & {$[196]$} & $1.44 \mathrm{E}-02$ & \\
Lyapunov Method $-D=3$ & {$[196]$} & $3.88 \mathrm{E}-02$ & \\
Lyapunov Method - Multi-dimension & {$[196]$} & $3.18 \mathrm{E}-04$ & \\
ERNN & {$[27]$} & & $6.45 \mathrm{E}-10$ \\
Simple Feedforward & & $3.98 \mathrm{E}-04$ & $3.00 \mathrm{E}-03$ \\
Proposed method - Elman & & $2.44 \mathrm{E}-11$ & $1.89 \mathrm{E}-10$ \\
Proposed method - Elman \& GA & & $1.62 \mathrm{E}-11$ & $1.21 \mathrm{E}-10$ \\
\hline
\end{tabular}


For the Henon time series, the performance of the proposed forecasting method in this study is compared with the results of a simple feedforward neural network and the results obtained from support vector regression (SVR), tapped delay line multi-layer perceptron (TDL-MLP), distributed local models (DLM), distributed local experts based on vector-quantization using information-theoretic concept (DLE-VQIT) [22], ensemble self-generating neural networks (ESGNN), GA merged with ensemble self-generating neural networks (GAESGNN) [156] and genetic wavelet neural networks (GWNN) [41]. The Elman recurrent neural network method improved with genetic algorithms exhibits the best results, compared to other methods, as shown in Table 4.8.

Table 4.8 Henon time series - comparing the prediction performance.

\begin{tabular}{llll}
\hline & & \multicolumn{2}{c}{ Prediction Error } \\
\hline Prediction Method & Reference & MSE & NMSE \\
\hline SVR & {$[22]$} & & $2.10 \mathrm{E}-02$ \\
TDL-MLP & {$[22]$} & $7.59 \mathrm{E}-02$ \\
DLM & {$[22]$} & $1.01 \mathrm{E}-01$ \\
DLE-VQIT & {$[22]$} & & $1.92 \mathrm{E}-02$ \\
ESGNN & {$[156]$} & & $2.23 \mathrm{E}-03$ \\
GAESGNN & {$[156]$} & & $2.01 \mathrm{E}-03$ \\
GWNN & {$[41]$} & $8.42 \mathrm{E}-05$ & \\
Simple Feedforward & & $3.2 \mathrm{E}-03$ & $5.8 \mathrm{E}-03$ \\
Proposed method - Elman & & $5.41 \mathrm{E}-09$ & $1.01 \mathrm{E}-08$ \\
Proposed method - Elman \& GA & & $4.76 \mathrm{E}-09$ & $9.01 \mathrm{E}-09$ \\
\hline
\end{tabular}




\subsection{Summary}

In this chapter, a combination of chaos theory and neural networks is used to develop a new technique to forecast chaotic time series. The phase space reconstruction method is employed to reconstruct phase space points from chaotic time series using embedding dimension $D$ and time delay $T$. According to the phase space reconstruction method, the phase space points will reproduce the key characteristics of the original time series. Artificial neural networks which can capture the relationships among the data where the underlying function is unknown or difficult to determine have been utilized to forecast the future value of phase space points and consequently the chaotic time series.

The feedforward and Elman neural networks are selected for one step ahead forecasting. The developed technique has been applied to three different chaotic time series, Mackey-Glass, Logistic and Henon, to validate the effectiveness of the proposed technique. The proposed method is merged with genetic algorithms to improve the forecasting results. It has been observed that the new forecasting method improved with GA presents an outstanding result. The comparison between the result of the proposed technique and the results reported in the literature substantiates the effectiveness of the proposed method. 


\section{CHAPTER 5}

\section{CHAOTIC TIME SERIES FORECASTING WITH RESIDUAL ANALYSIS USING ENSEMBLE NEURAL NETWORKS}

In this chapter, the contribution of residual analysis to improve the performance of the proposed forecasting method in Chapter 4 is investigated. A combination of the embedding theorem, artificial neural networks, residual analysis and genetic algorithms is employed to develop an effective technique to forecast chaotic time series. Similar to the previous method, the embedding theorem is utilized to unfold the chaotic time series and reconstruct the phase space points. The first artificial neural network is trained to forecast the future values of the phase space points and accordingly the original time series.

In the new approach, the residuals of the predicted time series are analyzed. In some events, the residuals show a high degree of correlation and demonstrate chaotic behaviour. Therefore, the residuals are considered as a new chaotic time series and reconstructed according to the embedding theorem. A new neural network is trained to predict the future values of the residual time series. The residual analysis can be repeated several times. Finally, a neural network is trained using the neural networks' result values of the time series and the residuals as input and the original time series as output. The last neural network is used to capture the relationship between the outcome of neural networks and the original time series. The weights and biases of the final artificial neural 
network are kept to predict the future values of the original time series. Two different configurations are considered in the selection of artificial neural network combinations. In the first configuration, all the selected neural networks are feedforward neural networks. In the second configuration, the combination of Elman and NARX neural networks is selected. The new proposed forecasting method is applied to the MackeyGlass and Lorenz equations which produce chaotic time series, and to a real life chaotic time series, sunspot time series, to evaluate the validity of the proposed technique. The experimental results confirm that the proposed method can predict the chaotic time series more effectively and accurately when compared with the existing prediction methods.

The content of the current chapter has been presented at the 29th International Symposium on Forecasting [199], submitted to Applied Artificial Intelligence Journal [200], and published in the Proceedings of the Industrial Engineering Research Conference [201] and the journal of Neurocomputing [202].

\subsection{Introducing Residual Analysis Method}

Heuristic models which are developed to predict time series mainly overlook the characteristics of the residuals. In some cases, heuristic models cannot completely capture the characteristics of the original time series, and the residuals show a high correlation. The high correlation of the residuals means that prediction errors are not due to randomness. This study considers this crucial finding and this section introduces a new technique which employs a combination of the embedding theorem, artificial neural networks, genetic algorithms and residual analysis to develop more effective method to forecast one step ahead of a chaotic time series. 
Figure 1.1 shows the block diagram demonstrating the proposed methodology in this chapter. Block A in Figure 1.1 is discussed earlier in Chapter 4. it proposes a forecasting method based on the embedding theorem and artificial neural networks. Block B in Figure 1.1 proposes a new approach to enhance the results of the forecasting method proposed in Chapter 4.

According to the Block A in Figure 1.1, the existence of chaos is investigated in the observed time series data set, $x(1), x(2), x(3), \ldots, x(N)$, where $N$ is the length of the data set. When the presence of chaos is detected in the observed time series, then the phase space parameters, embedding dimension $D$ and time delay $T$, are calculated. Based on the discussion in Section 3.2.3, the phase space points are generated. An artificial neural network with a single phase space point, $D$ input units, and a single phase space point, $D$ output units, is trained. After training the artificial neural network, the weights and biases of the network are kept to forecast the unknown phase space points. Finally the future values of the time series are obtained.

So far, one step ahead of the original time series is predicted. As mentioned earlier, in some cases heuristic models cannot completely capture the characteristics of the original time series and therefore, the residuals are highly correlated. The residuals resulting from the developed prediction method are analyzed, and it has been observed that in some cases they demonstrate chaotic behaviour.

To investigate the contribution of the residual analysis in improving the performance and accuracy of the proposed prediction method, the obtained residuals are considered as a new chaotic time series and analyzed separately. Block B in Figure 1.1 demonstrates the required steps to analyze and incorporate the residuals into the proposed 
forecasting method. The residuals obtained from the original prediction form a time series, $e_{1}(1), e_{1}(2), \ldots, e_{1}\left(N_{1}\right)$, where $N_{1}=N-(D-1) T-1$ is the length of the residual time series. The chaotic behaviour of the residual time series is investigated using the methods mentioned in Section 3.2.2. When the presence of chaos is confirmed in the residual time series, the embedding dimension $D_{1}$ and the time delay $T_{1}$ will be determined and the phase space points, $Y_{1}\left(j_{1}\right), j_{1}=1 \sim\left[N_{1^{-}}\left(D_{1^{-}}-1\right) T_{1}\right]$, will be generated as shown in Eq. (31).

$Y_{1}(1)=\left[e_{1}(1), e_{1}\left(1+T_{1}\right), \ldots, e_{1}\left(1+\left(D_{1}-1\right) T_{1}\right)\right]$ $Y_{1}(2)=\left[e_{1}(2), e_{1}\left(2+T_{1}\right), \ldots, e_{1}\left(2+\left(D_{1}-1\right) T_{1}\right)\right]$

$$
Y_{1}\left(j_{1}\right)=\left[e_{1}\left(N_{1}-\left(D_{1}-1\right) T_{1}\right), e_{1}\left(N_{1}-\left(D_{1}-1\right) T_{1}+T_{1}\right), \ldots, e_{1}\left(N_{1}\right)\right]
$$

A new artificial neural network with $D_{1}$ input units and $D_{1}$ output units is trained using the phase space points as input, $Y_{1}\left(i_{1}\right), i_{1}=1 \sim\left[N_{1^{-}}\left(D_{1}-1\right) T_{1}-1\right]$ and output $Y_{1}\left(k_{1}\right), k_{1}=$ 2 [N$\left.N_{1^{-}}\left(D_{1}-1\right) T_{1}\right]$. The input and output of the neural network are denoted by Eq. (32) and Eq. (33), respectively

$$
\begin{aligned}
& {\left[\begin{array}{l}
Y_{1}(1) \\
Y_{1}(2) \\
\cdot \\
\cdot \\
Y_{1}\left(N_{1}-\left(D_{1}-1\right) T_{1}-1\right)
\end{array}\right]} \\
& {\left[\begin{array}{l}
Y_{1}(2) \\
Y_{1}(3) \\
\cdot \\
\cdot \\
Y_{1}\left(N_{1}-\left(D_{1}-1\right) T_{1}\right)
\end{array}\right]}
\end{aligned}
$$


When the network training is completed, based on the known vector, $Y_{1}\left(N_{1^{-}}\left(D_{1^{-}}\right.\right.$ 1) $\left.T_{1}\right)$ as the input to the trained neural network, the unknown vector, $Y_{1}\left(N_{1}-\left(D_{1}-1\right) T_{1}+1\right)$ is predicted. $Y_{1}\left(N_{1^{-}}\left(D_{1}-1\right) T_{1}+1\right)$ is composed of known values $e_{1}\left(N_{1^{-}}\left(D_{1^{-}}-1\right) T_{1}+1\right), e_{1}\left(N_{1^{-}}\left(D_{1^{-}}\right.\right.$ 1) $\left.T_{1}+1+T_{1}\right), \ldots, e_{1}\left(N_{1}-\left(D_{1}-1\right) T_{1}+1+\left(D_{1}-2\right) T_{1}\right)$ and unknown value $e_{1}\left(N_{1}+1\right)$. When $Y_{1}\left(N_{1}-\right.$ $\left.\left(D_{1}-1\right) T_{1}+1\right)$ is predicted, the only unknown value, $e_{1}\left(N_{1}+1\right)$, can be determined.

To correlate the prediction values of the time series and the residuals to the original time series, a new neural network is trained. The length of the residual time series prediction is $N_{1}-\left(D_{1}-1\right) T_{1}-1$; therefore, the last $N_{1^{-}}\left(D_{1}-1\right) T_{1}-1$ data from the original time series prediction and the original time series are selected. A neural network with two input units and one output unit is trained using the neural networks' result values of the time series and the residuals as input and the original time series as output. The prediction values of the original time series are denoted as $x^{\prime}(i)$ and the residual time series as $e_{1}^{\prime}(i)$. Figure 5.1 demonstrates the structure of a feedforward neural network which correlates the outcome of neural networks to the original values. In case the NARX network is selected to correlate the prediction values to the original time series, the structure of the neural network can be illustrated as shown in Figure 5.2.

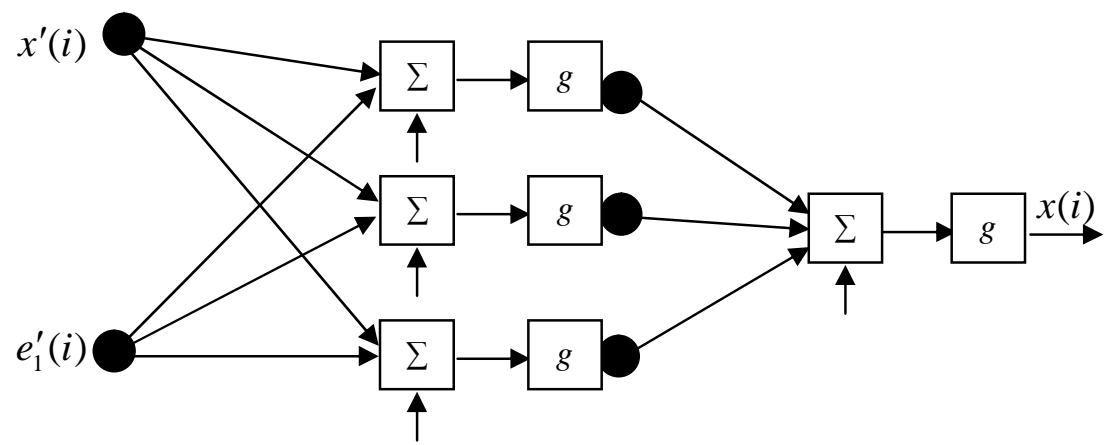

Figure 5.1 A FFNN correlating the original and residual time series prediction to original time series. 


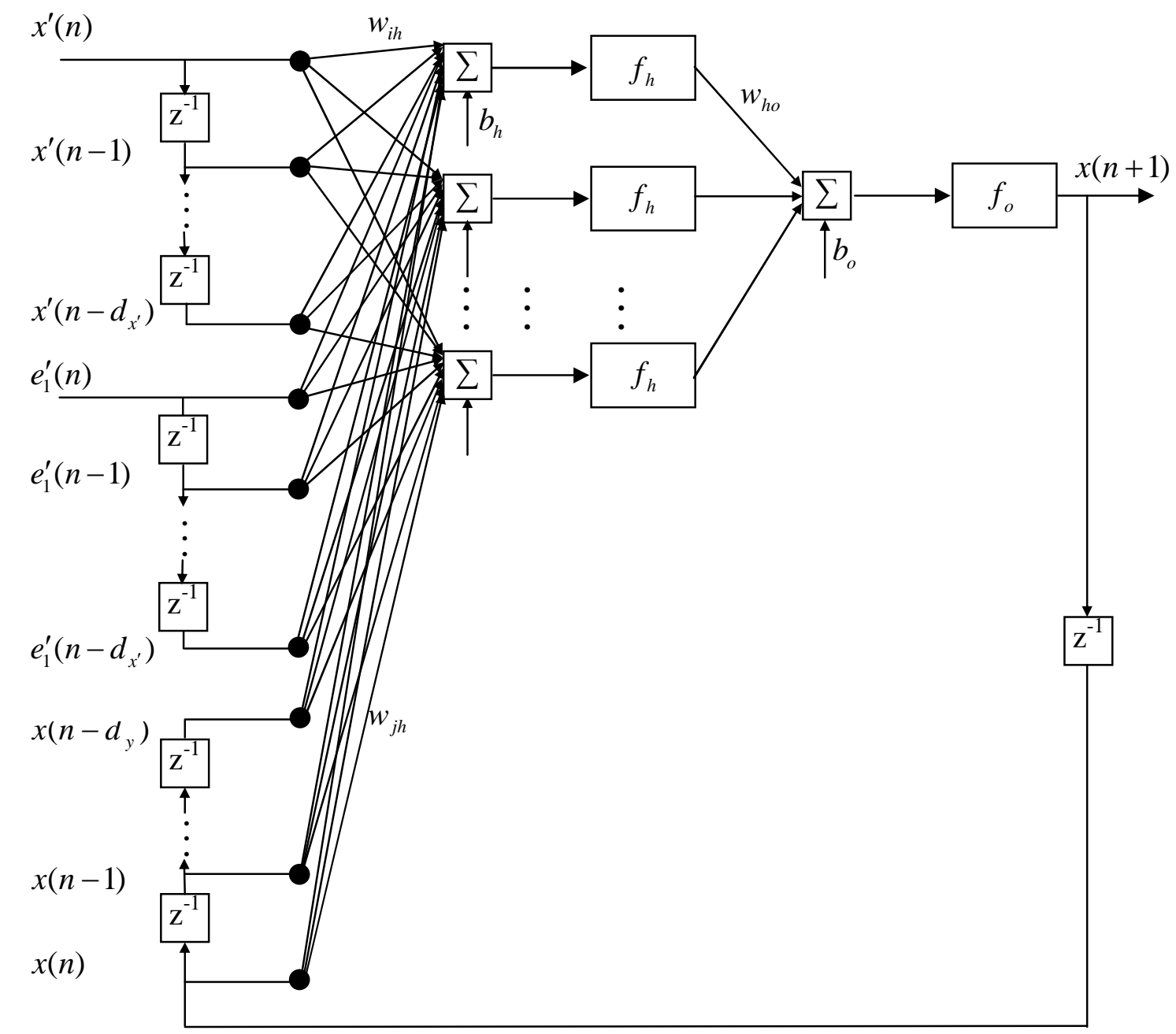

Figure 5.2 A NARX network correlating the original and residuals forecasts to original time series.

To increase the accuracy of the prediction, the residual analysis can be repeated for the second time. Thus, the residuals of the first residual time series prediction are analyzed further. The residuals of the first residual time series prediction are denoted as $e_{2}(1), e_{2}(2), \ldots, e_{2}\left(N_{2}\right)$, where $N_{2}=N_{2}-\left(D_{2}-1\right) T_{2}-1$. This time series will be analyzed and if it shows chaotic behaviour, it will be embedded into the phase space points. Later, the prediction method discussed in the beginning of this section will be applied to obtain the future values of the second residual time series, as $e_{2}^{\prime}(i)$. 
The residual analysis can be repeated several times. To correlate the neural networks' result values of the time series and the residuals in various levels to the original time series, a neural network with $M+1$ input units, where $M$ is the level of residual analysis, and one output unit can be trained. Figure 5.3 demonstrates the structure of the final neural network with $M$ level of residual analysis which correlates the prediction values to the original values. The proposed method is merged with genetic algorithms to improve the forecasting results.

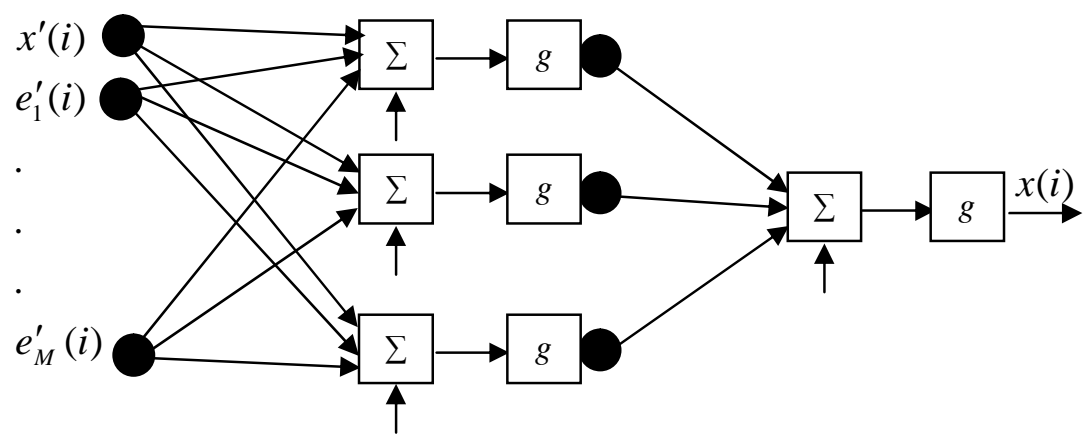

Figure 5.3 A FFNN correlating the original time series prediction and the prediction of residuals in various levels to original time series.

\subsection{Numerical Analysis Using Feedforward-Feedforward Configuration}

In this section, the unique approach developed in this chapter using feedforwardfeedforward neural network configuration is applied to two simulated chaotic systems: Mackey-Glass and Lorenz equations.

\subsubsection{Mackey-Glass Equation}

The details of the Mackey-Glass equation have been presented in Section 4.2.1. Similar to Section 4.2.1, the parameters in Eq. (26) are selected according to the previous report by Zhang and Man [1], where the constants are taken to be $a=0.2, b=0.1$ and $c=10$ and chaotic time series is generated by time delay $\tau=17$ and initial value $x(0)=1.2$. 
A chaotic time series data set with length of 1000 is generated by Eq. (26). The first 500 data are used as training data, while the remaining 500 are used to test the proposed model. The embedding dimension $D$ and the time delay $T$ are calculated using TSTOOL [190]. The original time series is reconstructed into phase space points with embedding dimension $D=3$ and time delay $T=7$. The data in the time domain and the phase space domain are shown in Figure 4.2.

As described in Section 5.1, the embedded phase space points are fed into a feedforward neural network with a single hidden layer and trained. The neural network has been set up with $D=3$ neurons in the input and output layers. The weights and biases of the feedforward neural network are kept to predict the unknown phase space points and eventually the future values of the time series. Figure 5.4 compares the real data and the predicated values for the remaining 500 samples kept for testing.

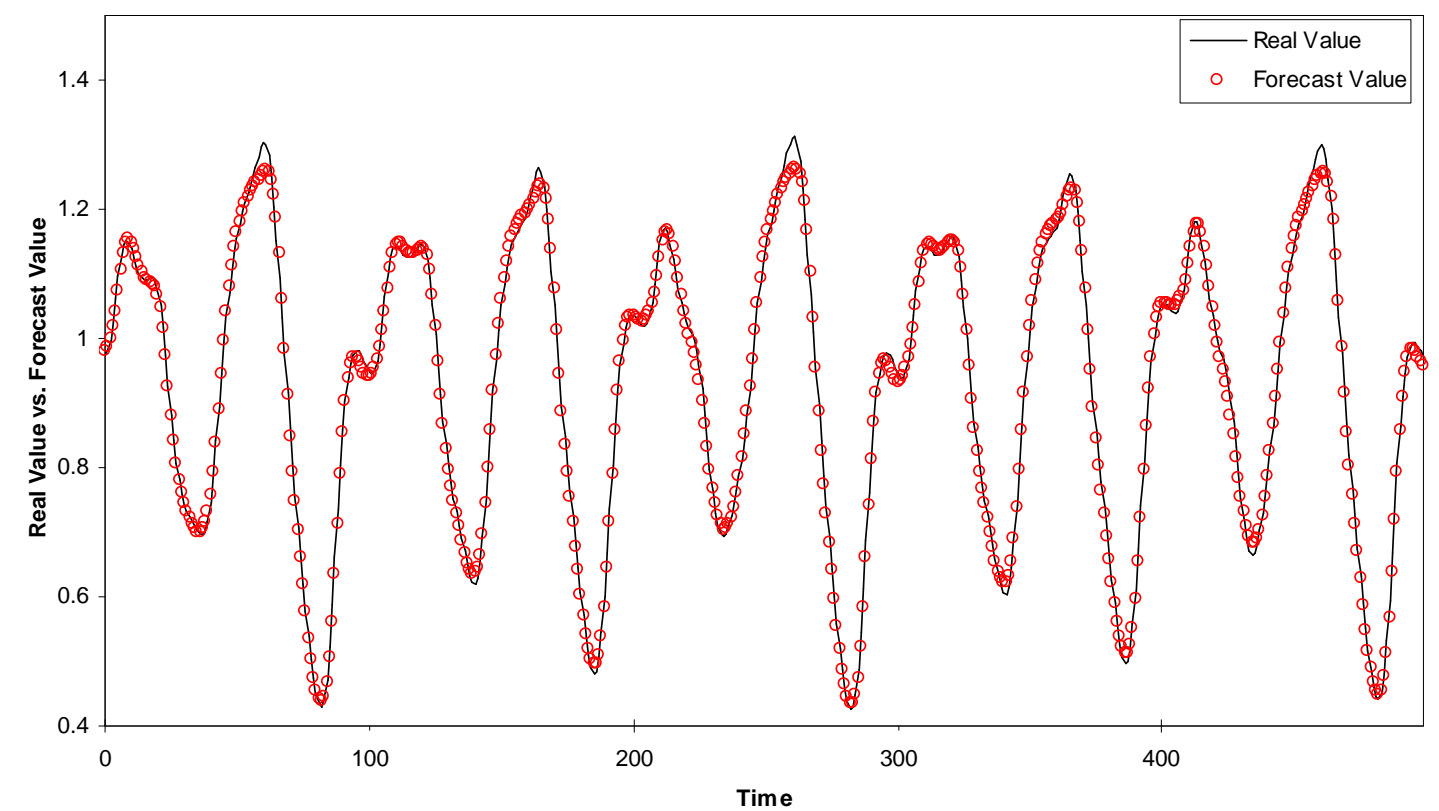

Figure 5.4 Mackey-Glass Time Series - Comparing the original time series data and the predicted values. 
The prediction errors for the training data are calculated and analyzed. The methods introduced in Section 3.2.2 are used to investigate the characteristic of the residuals. The power spectrum for the residuals is broadband and has a broad peak. The largest Lyapunov exponent (LLE) and fractal dimension (FD) for the time series are calculated using TSTOOL [190] and positive and non-integer, respectively. Based on a broadband power spectrum, positive LLE and non-integer FD, it is concluded that the residuals are chaotic. Therefore, it is concluded that the residuals show chaotic behaviour.

Based on this finding, the residuals are treated as a new chaotic time series and the prediction method proposed in Section 5.1 is applied to predict the residuals. The first $(D-1) T+1$ data has been used for the first prediction; therefore, the length of residual time series is $(D-1) T+1$ shorter than the original time series. Therefore, the 485 residuals obtained from original prediction of the training data are reconstructed using the estimated embedding dimension $D_{1}=3$ and time delay $T_{1}=3$. Figure 5.5 shows the residual time series in the time domain and the phase space domain. The calculated phase space points are fed into a new feedforward neural network.

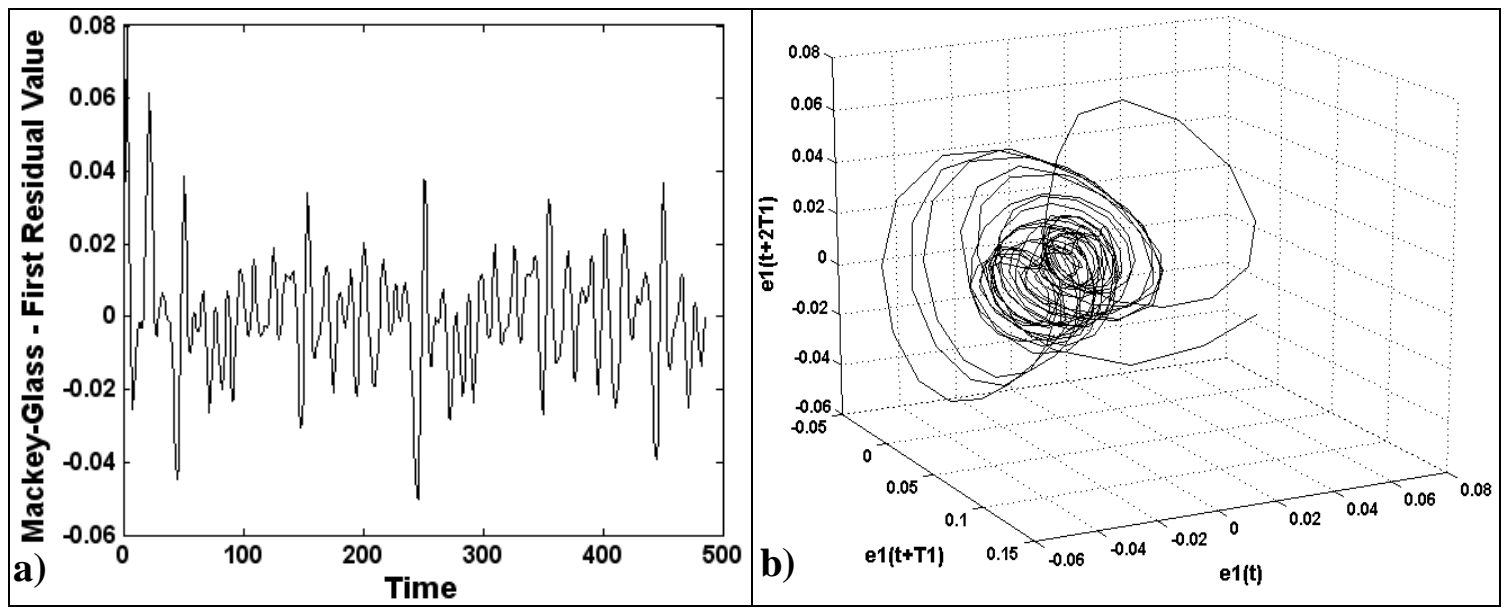

Figure 5.5 Mackey-Glass time series - First residual values a) in the time domain b) in the phase space domain. 
The neural network is set up with 3 neurons in the input and output layers. Figure 5.6 presents the first residual time series and their predicted values for the 500 test data.

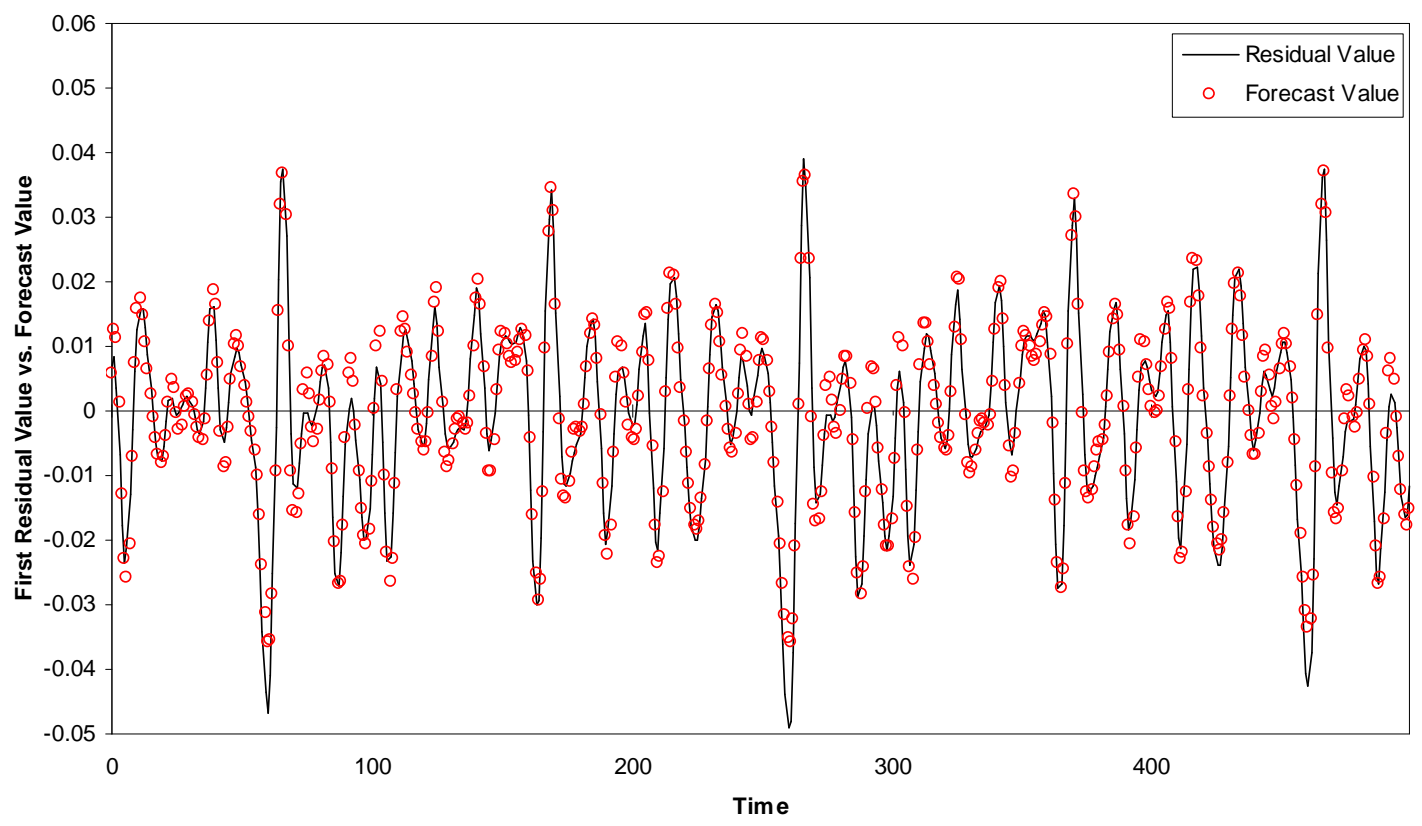

Figure 5.6 Mackey-Glass time series - The comparison of the first residual values and the predicted values.

The residual analysis is repeated one more time and the future values of the second order residuals are predicted based on the proposed forecasting method. Figure 5.7 demonstrates the residuals of the first residual prediction that is the second order residuals, in the time domain and the phase space domain, respectively. Figure 5.8 presents the comparison between the second order residual values and their predicted values.

Finally, a feedforward neural network is trained using the neural networks' result values of the time series and the residuals as input and the original time series as output. The last neural network is used to capture the relationship between the outcome of neural networks and the original time series. Similarly, the weights and biases of the final neural 
network are improved using genetic algorithms and kept to predict the future value of time series. Figure 5.9 compares the original values of the Mackey-Glass time series with their predicted values.

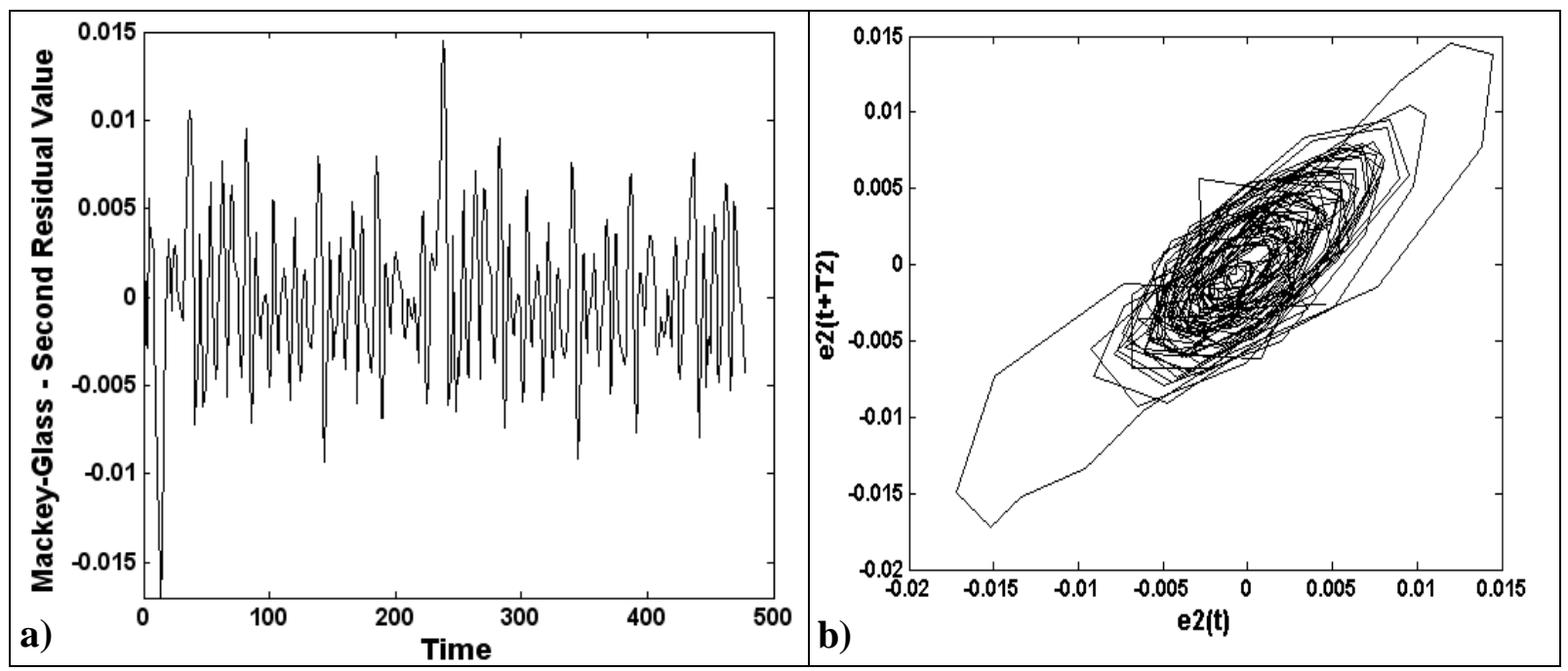

Figure 5.7 Mackey-Glass time series - The second residual values, a) in time domain b) in phase space domain.

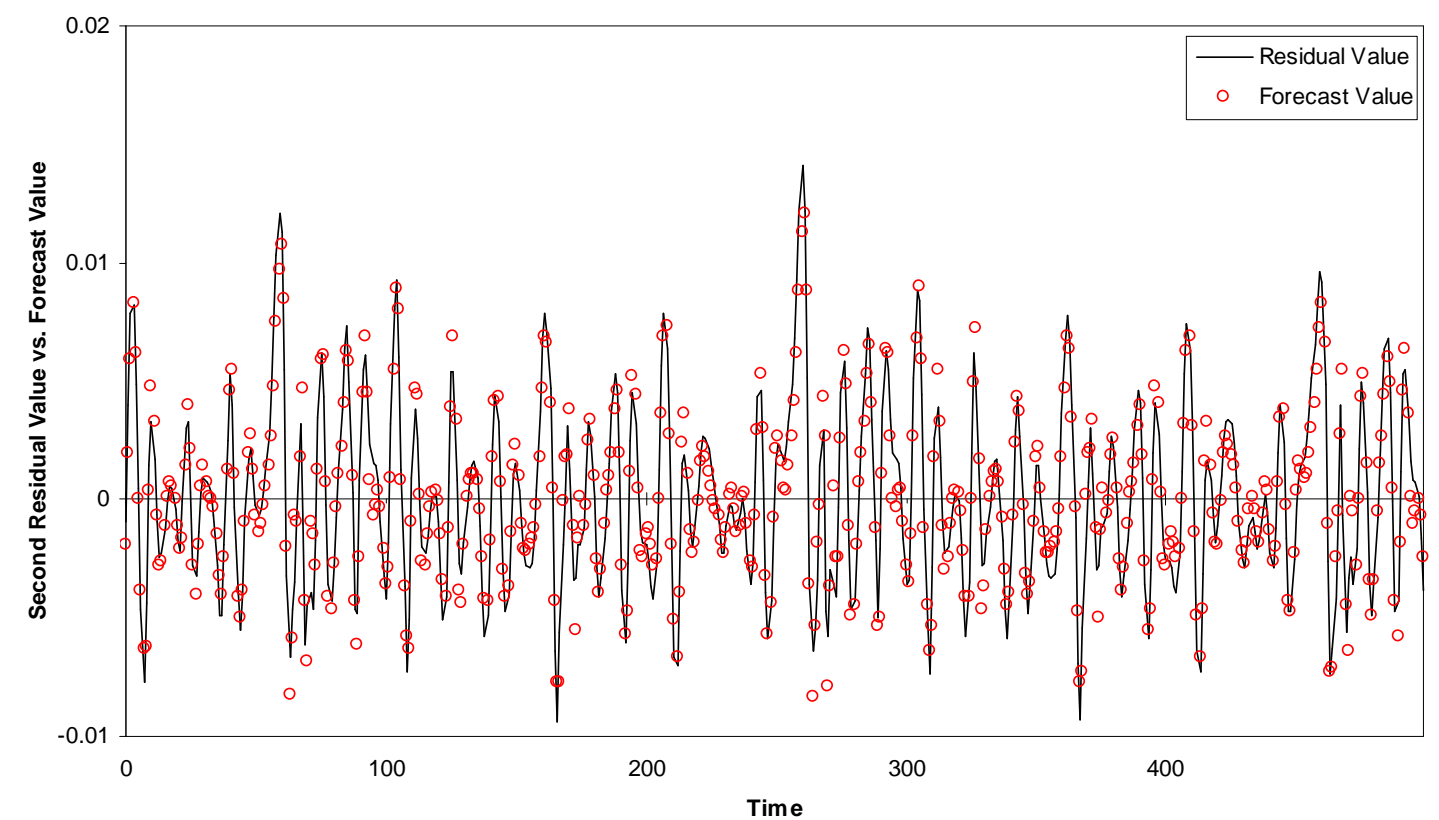

Figure 5.8 Mackey-Glass time series - The comparison of the second residual values and the predicted values. 


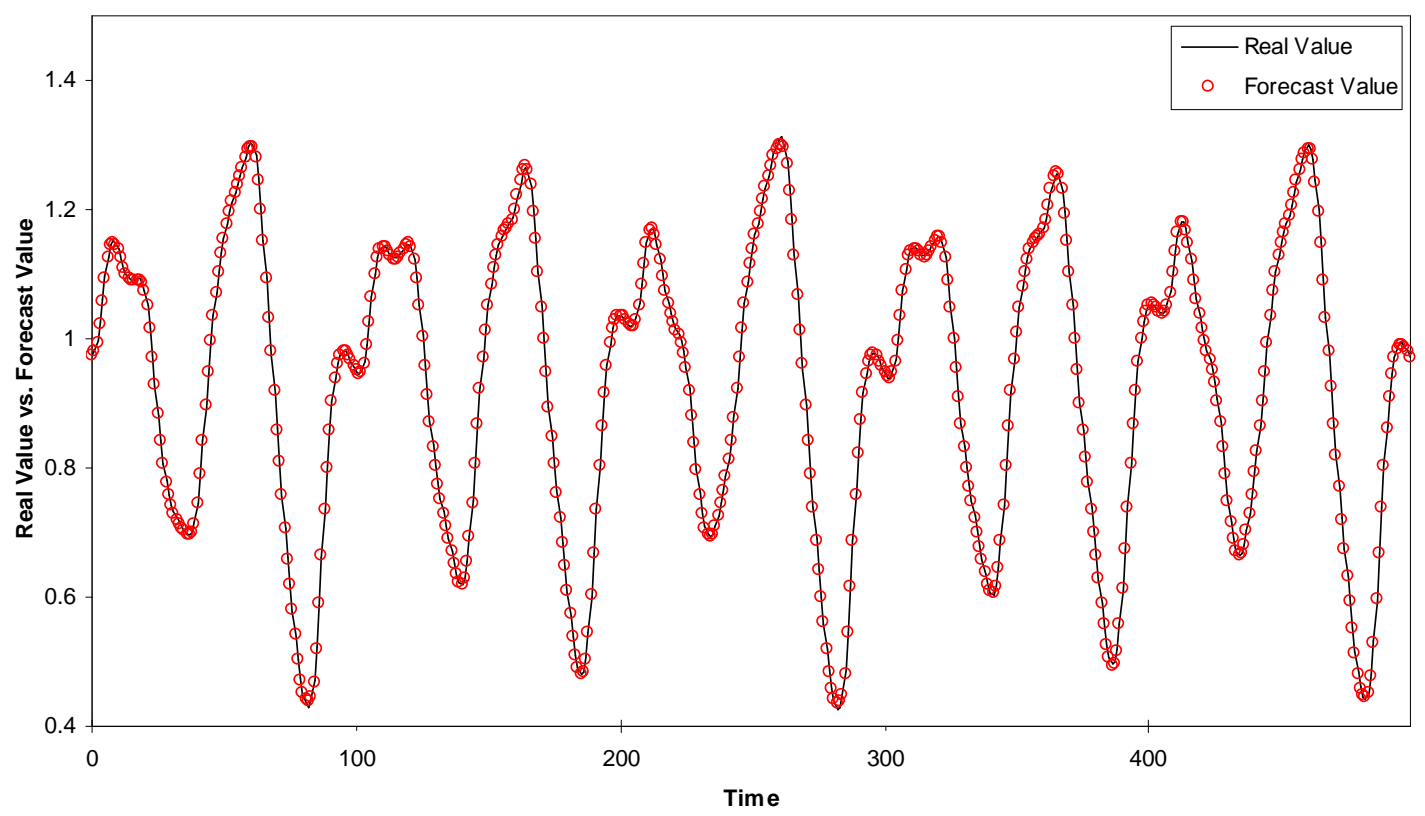

Figure 5.9 The comparison between original Mackey-Glass time series and the final predicted values.

In order to evaluate the prediction performance and compare it with the results reported in the literature, the mean squared error (MSE), normalized mean squared error (NMSE) and root mean squared error (RMSE) are calculated according to Eq. (27), Eq. (28) and Eq. (34), respectively.

$R M S E=\sqrt{\frac{1}{N} \sum_{i=1}^{N}\left(y_{i}-\hat{y}_{i}\right)^{2}}$

where $y_{i}, \hat{y}_{i}$ and $\bar{y}$ are observed data, predicted data and the average of observed data, respectively, and $N$ is the length of observed data. The obtained MSE, NMSE and RMSE are reflected in Table 5.1.

Table 5.1 Mackey-Glass time series - the error indices for FFNN-FNNN configuration.

Prediction Error

\begin{tabular}{llll}
\hline Prediction Method & MSE & RMSE & NMSE \\
\hline The Proposed Method - FFNN-FFNN & $1.82 \mathrm{E}-06$ & $1.30 \mathrm{E}-03$ & $3.53 \mathrm{E}-05$ \\
\hline
\end{tabular}




\subsubsection{Lorenz Equation}

An evident example of chaotic systems is the weather system. Lorenz's works have extensively contributed to the establishment of chaos theory [203]. The Lorenz equations are a simplified version of Navier-Stokes equations which are used in fluid mechanics. The Lorenz equations are written as Eq. (35):

$$
\begin{aligned}
& \frac{d x(t)}{d t}=\sigma[y(t)-x(t)] \\
& \frac{d y(t)}{d t}=x(t)[r-z(t)]-y(t) \\
& \frac{d z(t)}{d t}=x(t) y(t)-b z(t)
\end{aligned}
$$

where $\sigma, r$, and $b$ are dimensionless parameters and the typical values for these parameters are $\sigma=10, r=28$ and $b=8 / 3$ [27,84]. The $x$-coordinate of the Lorenz time series is considered for prediction and a time series with a length of 2500 is generated as described in the research work by Ma et al. [27]. The first 1500 samples were used as training data, while the remaining 1000 were used to test the proposed model. The embedding dimensions $D=3$ and $T=3$ are estimated as explained using TSTOOL [190], an add-on toolbox in MATLAB [191] and accordingly phase space points are reconstructed. The data in the time domain and phase space domain are shown in Figure 5.10.

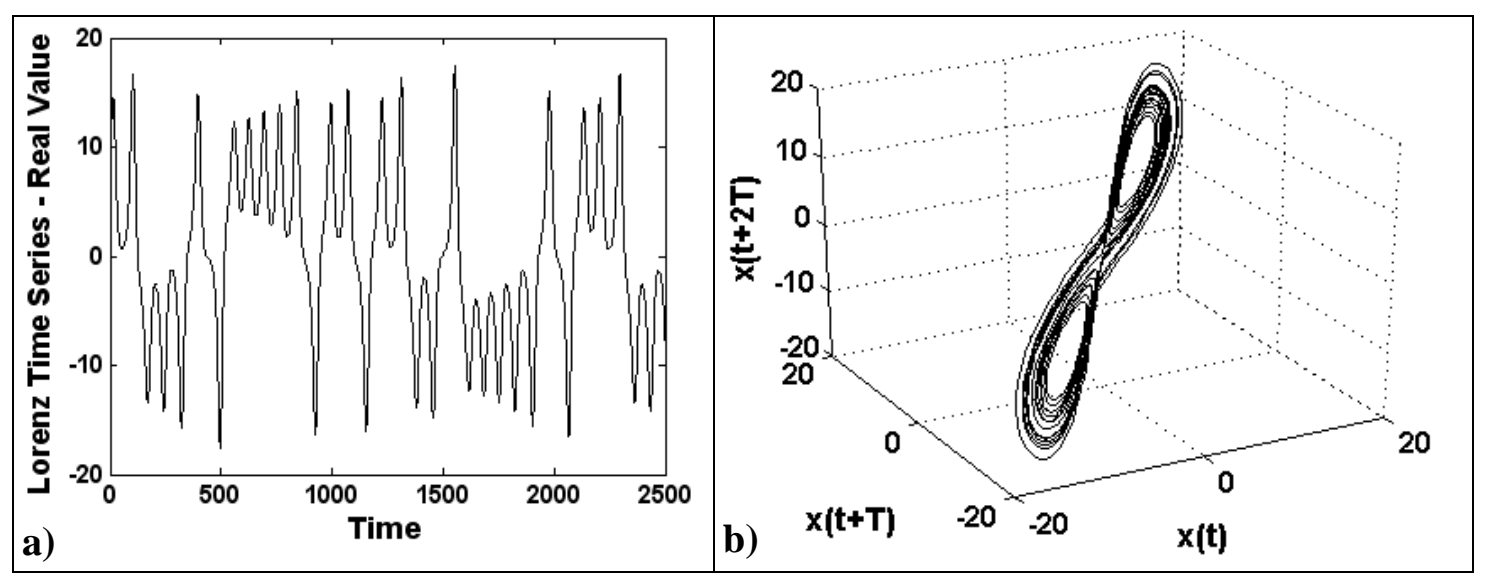

Figure 5.10 Lorenz time series, a) in time domain, b) in phase space domain. 
A feedforward neural network containing 3 neurons in the input layer and 3 neurons in the output layer is trained. The weights and biases of the network are kept for purpose of prediction. Figure 5.11 compares the real data and the predicated values for the remaining 1000 test samples using the feedforward neural network.

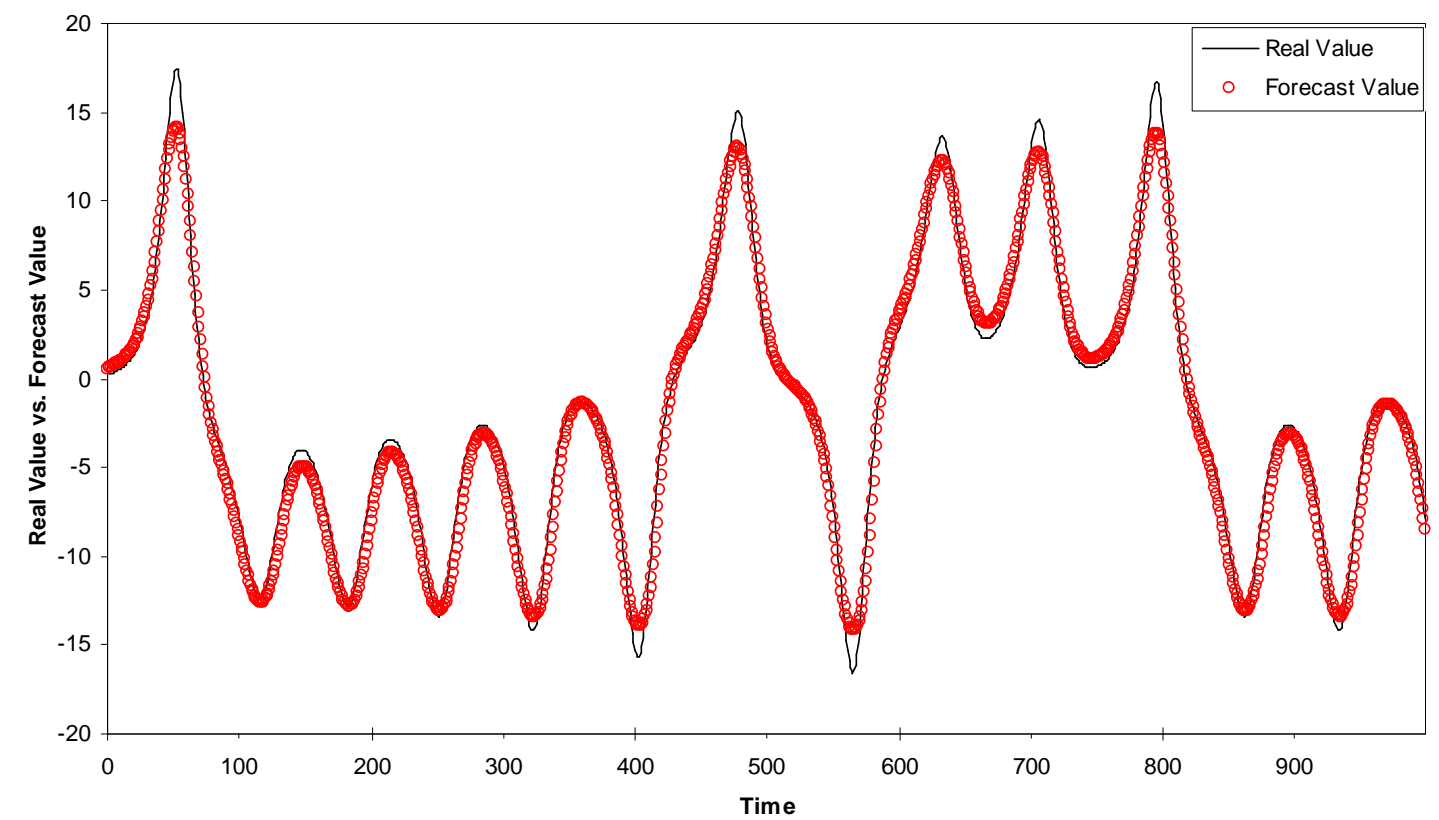

Figure 5.11 Lorenz time series - The comparison of the original time series samples and the predicted values based on feedforward network.

The prediction errors for the training data are calculated and analyzed. The methods introduced in Section 3.2.2 are used to investigate the characteristic of the residuals. The power spectrum for the residuals is broadband and has a broad peak. The largest Lyapunov exponent (LLE) is positive and fractal dimension (FD) for the residual time series is non-integer. Therefore, it is concluded that the residuals show chaotic behaviour.

Based on this finding, the residuals are treated as a new chaotic time series and the prediction method proposed in Section 5.1 is applied to predict the future values of the 
residuals. The first $(D-1) T+1$ observations will help to predict the first prediction; therefore, the length of residual time series is $(D-1) T+1$ shorter than the original time series. The residuals may not inherit the same characteristics as the original time series; therefore, they need to be analyzed independently. Therefore, 1493 samples are reconstructed with the new embedding dimension $D_{1}=3$ and time delay $T_{1}=3$ for the residual time series. Figure 5.12 shows the first residual time series in the time domain and the phase space domain. Figure 5.13 presents the results of the first order residuals prediction.

The second level of residual analysis is performed and the evidence of chaos is observed in the residuals. The second level of residuals are treated as a new time series and predicted using a new feedforward neural network.
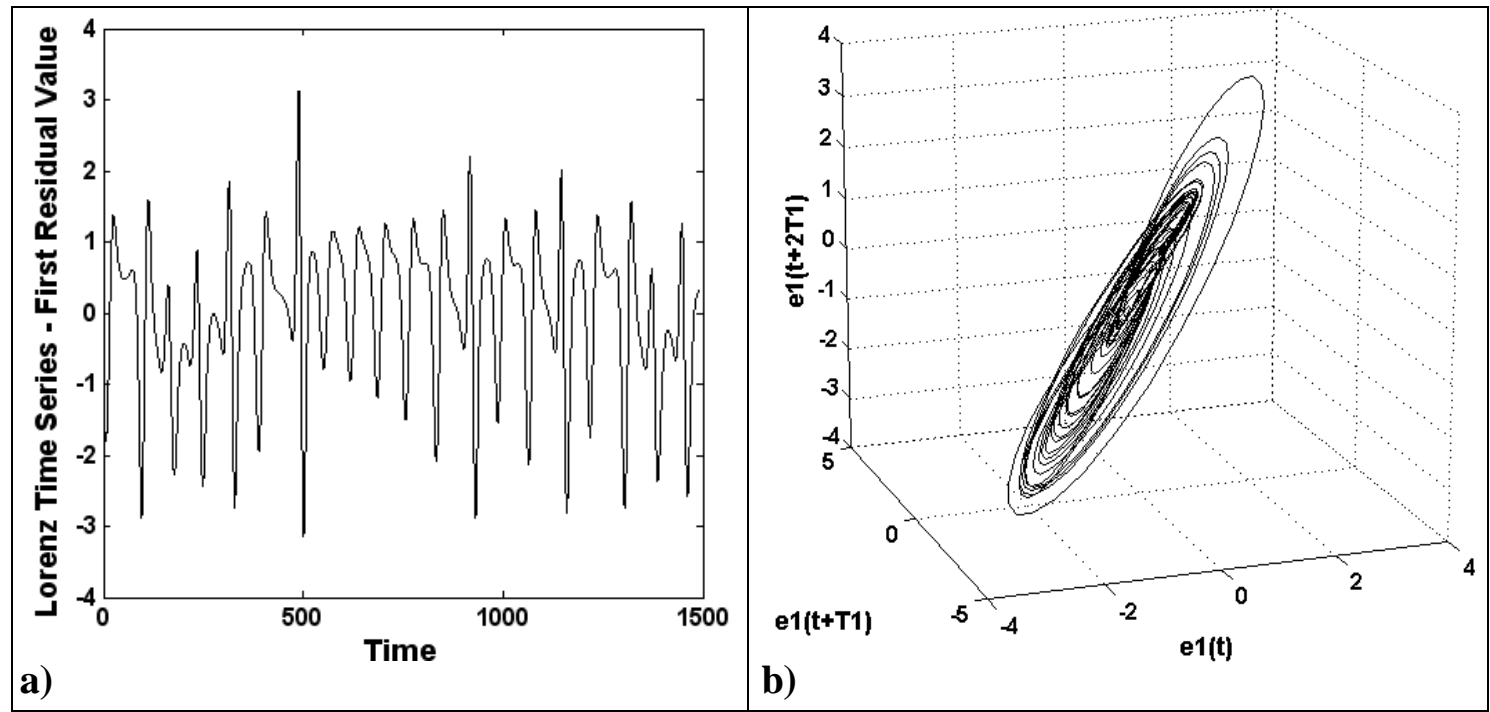

Figure 5.12 Lorenz time series - The first residuals a) in the time domain, b) in the phase space domain.

Finally, a feedforward network is trained using the neural networks' result values of the time series and the residuals as input and the original time series as output to 
correlate the outcome of neural networks to the original time series. Figure 5.14 compares the Lorenz time series with the predicted values.

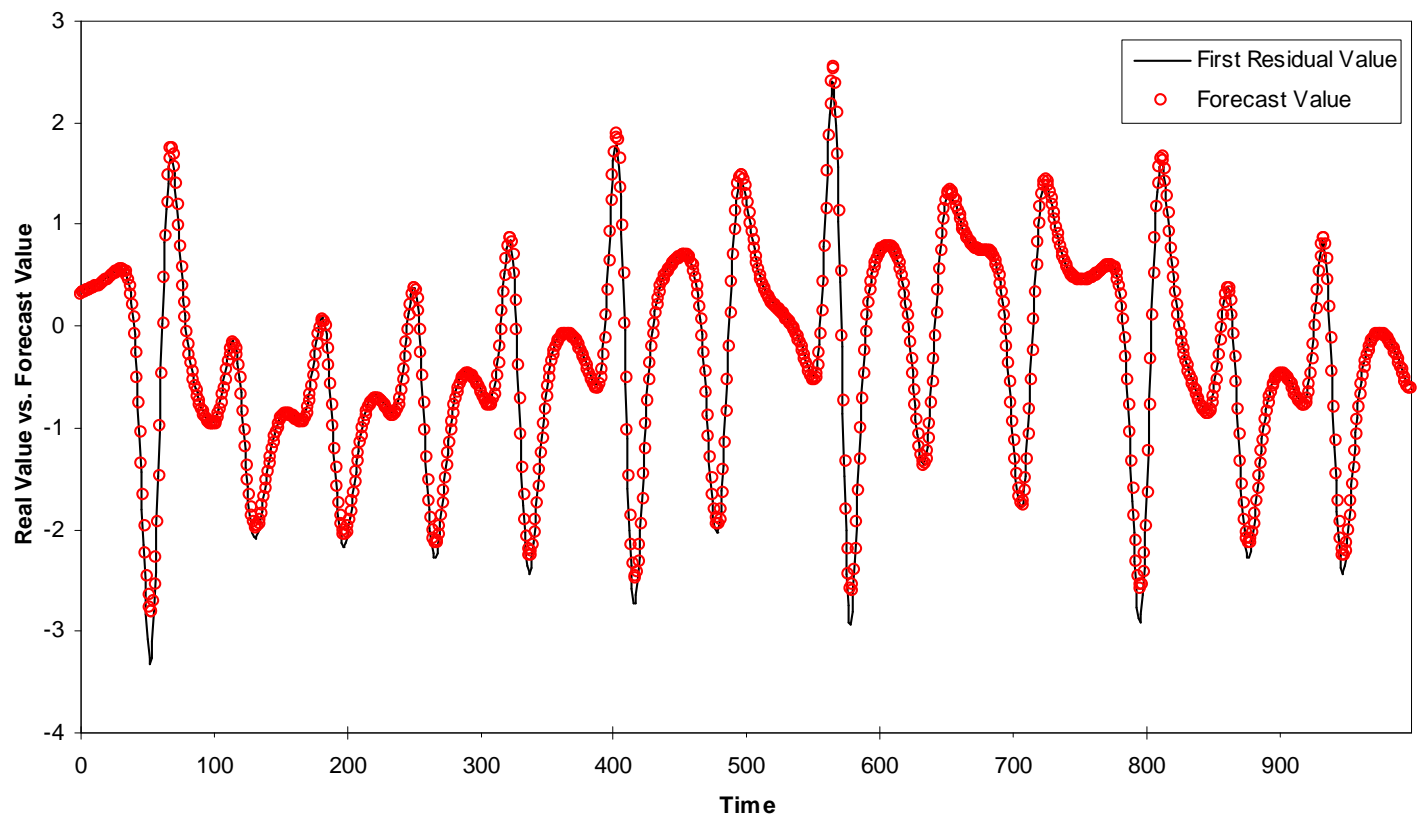

Figure 5.13 Lorenz time series - the comparison of the first residual and predicted values.

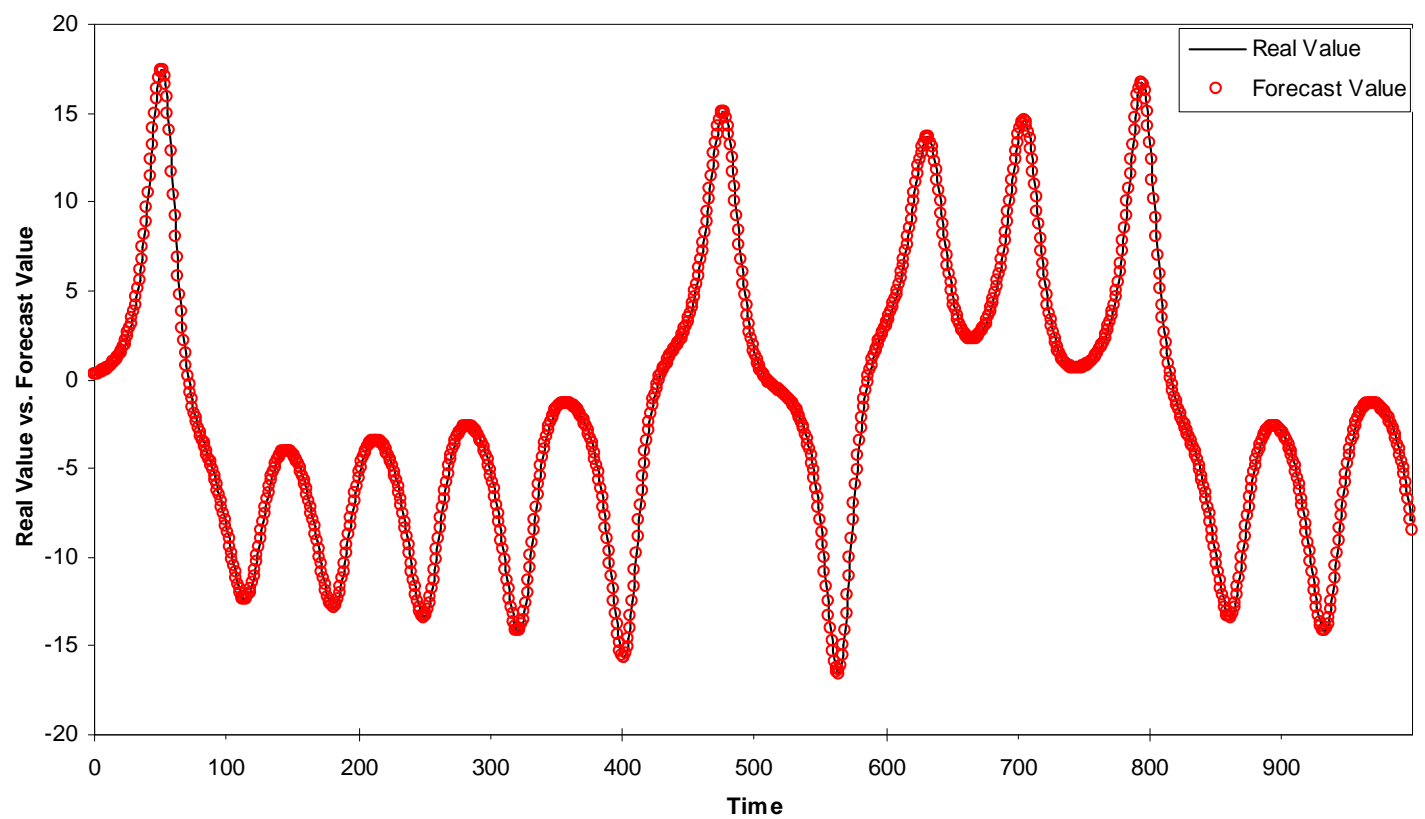

Figure 5.14 The comparison between original Lorenz time series and the final predicted values. 
In order to evaluate the prediction performance and compare it with the results reported in the literature, the mean squared error (MSE), normalized mean squared error (NMSE) and root mean squared error (RMSE) are calculated and listed in Table 5.2.

Table 5.2 Lorenz time series - the error indices for FFNN-FNNN configuration.

\begin{tabular}{llll}
\hline & \multicolumn{3}{c}{ Prediction Error } \\
\hline Prediction Method & $M S E$ & RMSE & NMSE \\
\hline The Proposed Method - FFNN-FFNN & $8.73 \mathrm{E}-04$ & $2.96 \mathrm{E}-03$ & $1.58 \mathrm{E}-05$ \\
\hline
\end{tabular}

\subsection{Numerical Analysis Using EIman-NARX Configuration}

The experiments in Section 5.2 which are performed with feedforwardfeedforward configuration are repeated with the Elman recurrent neural network to predict the time series and the residual time series and the NARX to correlate the outcome of the neural networks to the original time series. The conditions are kept the same to be able to compare the results. An additional experiment is performed on a real life chaotic time series, sunspot time series, in this section.

\subsubsection{Mackey-Glass Equation}

As stated in Section 5.2.1, a chaotic time series data set with length of 1000 is generated by the Mackey-Glass equation, Eq. (26), as described in the research work by Zhang and Man [1]. The first 500 data were used as training data, while the remaining 500 were used to test the proposed model. The original time series is reconstructed with embedding dimension $D=3$ and time delay $T=7$. The embedded phase space points are fed into an Elman network with 3 neurons in the input and output layers and 6 recurrent and 
hidden neurons and trained. The weights and biases of the Elman neural network are kept to forecast the future values of phase space points and consequently the original time series.

Two levels of residual analysis are performed to increase the accuracy of the prediction. The first order residuals, 485 samples, are reconstructed with embedding dimension $D_{1}=3$ and time delay $T_{1}=3$. Based on a broadband power spectrum, positive LLE and non-integer FD, it is concluded that the residuals are chaotic. The reconstructed phase space points are fed into a new Elman neural network. The neural network is set up with 3 neurons in the input and output layers and 10 neurons in the hidden layer. The residual analysis is repeated one more time with $D_{2}=3$ and time delay $T_{2}=3$ and after confirmation that the residuals are chaotic, the future values of the second order residuals are predicted based on the proposed prediction method. And finally, a NARX network is trained using neural networks' result values of the time series and the residuals as input and the original time series as output. Table 5.3 presents the prediction errors, MSE, RMSE and NMSE, based on the proposed method for 500 Mackey-Glass test samples.

Table 5.3 Mackey-Glass time series - the error indices for Elman-NARX configuration.

\begin{tabular}{llll} 
& \multicolumn{3}{c}{ Prediction Error } \\
\hline Prediction Method & $M S E$ & $R M S E$ & NMSE \\
\hline The Proposed Method - Elman-NARX & $1.3855 \mathrm{E}-09$ & $3.7222 \mathrm{E}-05$ & $2.7040 \mathrm{E}-08$ \\
\hline
\end{tabular}

\subsubsection{Lorenz Equation}

Similar to Section 5.2.2, the $x$-coordinate of the Lorenz time series is considered for prediction and a time series with a length of 2500 is generated as described in the research work by Ma et al. [27]. The first 1500 samples were used as training data, while the remaining 1000 were used to test the proposed model. The embedding dimensions $D=3$ 
and $T=3$ are estimated as explained in Sections 3.2.4 and 3.2.5 using TSTOOL [190], an add-on toolbox in MATLAB [191]. Accordingly, the phase space points are reconstructed. An Elman neural network including 3 neurons in the input layer, 6 neurons in the hidden layer, 6 recurrent neurons and 3 neurons in the output layer is trained. The weights and biases of the network are kept for prediction purposes.

The prediction errors for the data selected for the training phase are calculated and analyzed. The power spectrum for the residuals is broadband and has a broad peak. The largest Lyapunov exponent (LLE) and fractal dimension (FD) for the time series are calculated using TSTOOL [190] and they are positive and non-integer, respectively. Therefore, it is concluded that the residuals show chaotic behaviour.

Based on this finding, the residuals are treated as a new chaotic time series and the prediction method proposed in Section 5.1 is applied to predict the future values of the residuals. The first $(D-1) T+1$ observations help to predict the first prediction; therefore, the length of residual time series is $(D-1) T+1$ shorter than the original time series. The residual time series may not inherit the same characteristics as the original time series; therefore, they need to be analyzed independently. Therefore, 1493 samples are reconstructed with embedding dimension $D_{1}=3$ and time delay $T_{1}=1$. The second level of residual analysis is performed with embedding dimension $D_{2}=3$ and time delay $T_{2}=1$ and 1490 samples. Finally, a NARX network is trained using the neural networks' result values of the time series and the residuals as input and the original time series as output to correlate the outcome of the neural networks to the original time series. Table 5.4 presents the prediction errors, MSE, RMSE and NMSE, based on the proposed method for 1000 Lorenz test samples. 
Table 5.4 Lorenz time series - the error indices for Elman-NARX configuration.

\begin{tabular}{llll}
\hline & \multicolumn{3}{c}{ Prediction Error } \\
\hline Prediction Method & $M S E$ & $R M S E$ & NMSE \\
\hline The Proposed Method - Elman-NARX & $1.1731 \mathrm{E}-08$ & $1.0831 \mathrm{E}-04$ & $1.983 \mathrm{E}-10$ \\
\hline
\end{tabular}

\subsubsection{Sunspot Time Series}

Forecasting solar activity is a challenging area and important topic for various researchers and industries [82]. The sunspot time series is a good indication of solar activity for solar cycles. The impact of solar activity has been observed on earth, climate, weather, satellites and space missions; therefore, it is critical to forecast the sunspot time series. However, because of the complexity of the system and the lack of a mathematical model, forecasting the solar cycle is extremely challenging [84].

The monthly smoothed sunspot time series has been obtained from the SIDC (World Data Center for the Sunspot Index) [204]. To compare the results with some of the research works published in the literature, data are selected in the same conditions reported by Ma et al. [27] and Gholipour et al. [84]. The sunspot series from November 1834 to June 2001 (2000 points) are selected and scaled between [0,1]. The first 1000 samples of time series are selected to train and the remaining 1000 samples are kept to test the prediction method. The parameters $D=5$ and $T=1$ are estimated and accordingly the phase space points are reconstructed. The Elman neural network consists of 5 neurons in the input layer, 7 neurons in the hidden layer, 7 recurrent neurons and 5 neurons in the output layer. The network is trained using a gradient descent with momentum and adaptive 
learning rate backpropagation algorithm. Figure 5.15 compares the real data and the predicated values for the remaining 1000 test samples using the Elman neural network.

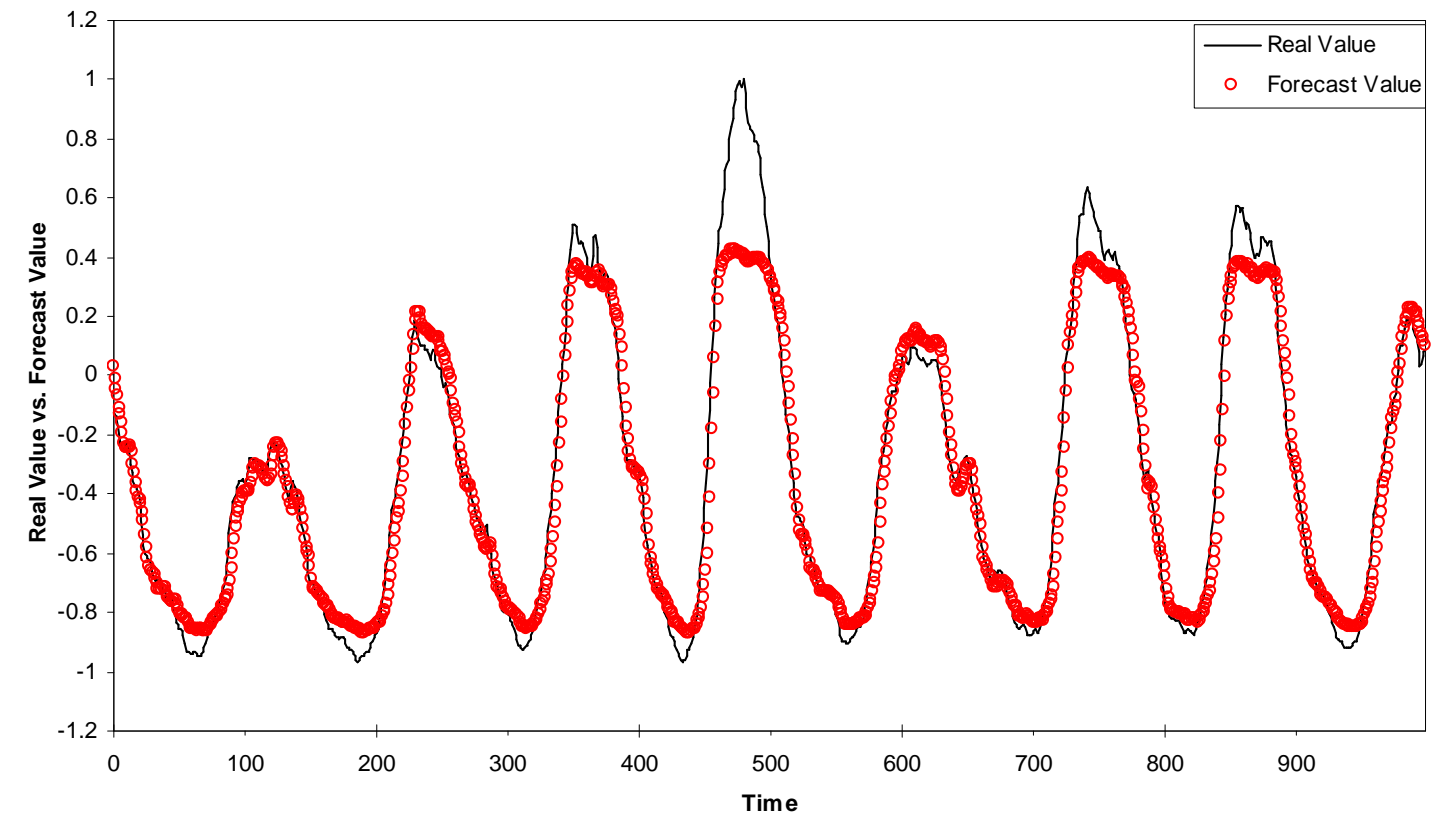

Figure 5.15 Sunspot time series - The comparison of the original time series samples and the predicted values based on Elman network.

The prediction errors for the samples selected for the training are calculated and analyzed. The power spectrum is broadband with a broad peak and the calculated LLE and FD are positive and non-integer, respectively. Therefore, the residuals show chaotic behaviour. The 995 residual points are reconstructed with embedding dimension $D_{1}=5$ and time delay $T_{1}=1$. Similarly, the second Elman neural network is trained using a gradient descent with momentum and adaptive learning rate backpropagation algorithm. Another level of the residual analysis is performed to increase the accuracy of the prediction. The residual analysis shows that the second order residuals are chaotic too; therefore, 990 residual points are reconstructed with embedding dimension $D_{2}=3$ and time delay $T_{2}=1$. Finally, a NARX network is 
trained using the neural networks' result values of the time series and the residuals as input and the original time series as output to correlate the predicted values to the original time series. Figure 5.16 compares the original values of the sunspot time series with their predicted values. The error indices, MSE, RMSE and NMSE, for the 1000 sunspot time series test samples are presented in Table 5.5.

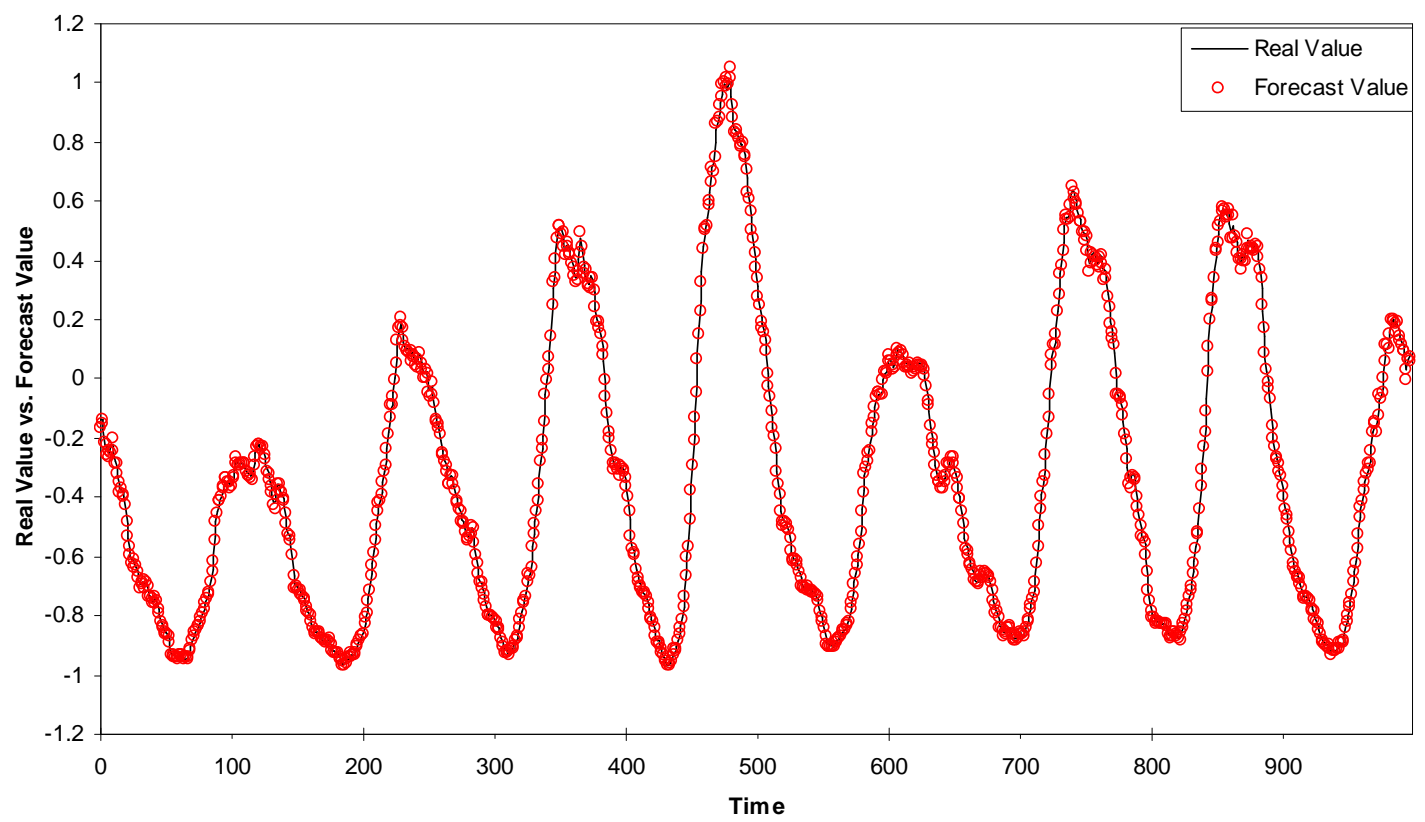

Figure 5.16 The comparison between original sunspot time series and the final predicted values.

Table 5.5 Sunspot time series - the error indices for Elman-NARX configuration.

\begin{tabular}{llll} 
& \multicolumn{3}{c}{ Prediction Error } \\
\hline Prediction Method & $M S E$ & RMSE & NMSE \\
\hline The Proposed Method - Elman-NARX & $1.4078 \mathrm{E}-04$ & $1.19 \mathrm{E}-02$ & $5.9041 \mathrm{E}-04$ \\
\hline
\end{tabular}




\subsection{Comparing the Results}

To evaluate the proposed forecasting methods both in feedforward and ElmanNARX configurations, the prediction accuracy of the methods when applied to the Mackey-Glass, Lorenz and sunspot time series as benchmarking are compared to the prediction performance of the methods presented in the literature.

For the Mackey-Glass time series, the performance of the proposed method in this chapter is compared with the results reported in the literature. Wang and Mendel [205] developed a forecasting method which generates fuzzy rules from sample data. In the paper published by Cho and Wang [115], a method based on a neuro-fuzzy system with adaptive capability to extract fuzzy if-then rules from input and output numerical data through learning is suggested. The method by Cho and Wang [115] which is called radial basis function (RBF) based adaptive fuzzy system (AFS) used Gaussian functions as the membership functions of the premise portion of fuzzy rules. Also, Jang et al. [145] and Zhang et al. [39] used neuro-fuzzy method to forecast chaotic time series.

In the research work by Rojas et al. [116], a framework to configure and train a radial basis function $(\mathrm{RBF})$ is introduced. A sequential learning algorithm is adopted to organize the structure of the network. Pseudo-Gaussian function (PG) is used to modify the structure of the Gaussian functions. Rojas et al. [31] developed an expert system based on artificial intelligence in which the parameters of the ARMA model could be determined automatically. In this study, a hybrid forecasting method was introduced based on the artificial neural network and ARMA.

Kim and Kim [154] proposed a genetic fuzzy predictor ensemble (GFPE) method to predict the future of chaotic time series. Gholipour et al. [84] examined several neural 
and neuro-fuzzy models with different learning algorithms for prediction of several benchmark chaotic time series. The performance of locally linear neuro-fuzzy models with the developed locally linear model tree (LoLiMoT) learning algorithm is compared with the prediction performance of radial basis function (RBF) neural network with orthogonal least squares (OLS) learning algorithm, multi-layer perceptron neural network with error backpropagation learning algorithm, and adaptive network based fuzzy inference system.

Teo et al. [122] used a wavelet packet multi-layer perceptron neural network (WP-MLP) trained by backpropagation learning method for prediction of chaotic time series. Zhang and Man [1] developed a multi-dimension chaotic time series prediction method using recurrent neural network (RNN) in embedding phase space. Similarly, Assaad et al. [107] proposed a forecasting method based on boosted recurrent neural networks. Inoue et al. [206] used hybrid neural networks to forecast chaotic time series.

Table 5.6 presents the comparison between the prediction errors, MSE, RMSE and NMSE, reported in the literature and the proposed method for 500 Mackey-Glass test samples. The results in Table 5.6 are sorted based on RMSE error index. Although ERNN method shows close results compared to the proposed method for Mackey-Glass time series, in other benchmarking time series the proposed forecasting method supersedes ERNN method. The results show that the proposed forecasting method with Elman-NARX configuration performs better in prediction of the Mackey-Glass chaotic time series when compared to other prediction methods reported in the literature. 
Table 5.6 Comparison between the prediction errors reported in the literature and the proposed method (500 Mackey-Glass time series test samples).

\begin{tabular}{|c|c|c|c|c|}
\hline \multirow[b]{2}{*}{ Prediction Method } & \multirow[b]{2}{*}{ Reference } & \multicolumn{3}{|c|}{ Prediction Error } \\
\hline & & $M S E$ & $R M S E$ & NMSE \\
\hline Auto-Regressive Model & {$[116]$} & & $1.90 \mathrm{E}-01$ & \\
\hline Product T-Norm & {$[205]$} & & $9.10 \mathrm{E}-02$ & \\
\hline Min T-Norm & {$[205]$} & & $0.90 \mathrm{E}-02$ & \\
\hline Cascade Correlation Neural Network & {$[116]$} & & $0.60 \mathrm{E}-02$ & \\
\hline Genetic Algorithms and Fuzzy System - 5 MFs & {$[154]$} & & 4.90E-02 & \\
\hline Genetic Algorithms and Fuzzy System - 7 MFs & {$[154]$} & & $4.20 \mathrm{E}-02$ & \\
\hline Six-order Polynomial & [116] & & 4.0E-02 & \\
\hline Genetic Algorithms and Fuzzy System - 9 MFs & {$[154]$} & & $3.80 \mathrm{E}-02$ & \\
\hline Radial Basis Function - Adaptive Fuzzy System III & {$[115]$} & & $1.31 \mathrm{E}-02$ & \\
\hline Radial Basis Function - Adaptive Fuzzy System II & {$[115]$} & & $1.28 \mathrm{E}-02$ & \\
\hline Radial Basis Function - Adaptive Fuzzy System I & {$[115]$} & & $1.14 \mathrm{E}-02$ & \\
\hline ANFIS and Fuzzy System & {$[145]$} & & $7.00 \mathrm{E}-03$ & \\
\hline Pseudo Gaussian - Radial Basis Function & {$[116]$} & & $2.80 \mathrm{E}-03$ & \\
\hline ARMA - Neural Network & {$[31]$} & & $2.50 \mathrm{E}-03$ & \\
\hline ANFIS & {$[84]$} & & $1.50 \mathrm{E}-03$ & \\
\hline RBF-OLS & {$[84]$} & & $1.02 \mathrm{E}-03$ & \\
\hline LLNF-LoLiMot & {$[84]$} & & $9.61 \mathrm{E}-04$ & \\
\hline ERNN & {$[27]$} & & $4.20 \mathrm{E}-05$ & $3.15 \mathrm{E}-08$ \\
\hline Recurrent Neural Network $-\mathrm{D}=3$ & {$[1]$} & 4.15E-04 & & \\
\hline Recurrent Neural Network - Multi-Dimension & [1] & $3.81 \mathrm{E}-04$ & & \\
\hline WP-MLP (A) & {$[122]$} & $1.25 \mathrm{E}-06$ & & \\
\hline Neuro-Fuzzy System with Delay & [39] & & & $1.26 \mathrm{E}-03$ \\
\hline BRNN & {$[107]$} & & & $1.60 \mathrm{E}-04$ \\
\hline Hybrid Neural Network (HNN) & [206] & & & $5.30 \mathrm{E}-02$ \\
\hline Proposed Method - Elman-NARX & & $1.39 \mathrm{E}-09$ & $3.72 \mathrm{E}-05$ & 2.70E-08 \\
\hline
\end{tabular}


The prediction performance for the Lorenz time series based on the proposed method in this chapter is compared with the same of the forecasting methods presented in the research works in the literature. The performance of the forecasting methods by Jang et al. [145], Rojas et al. [116], Rojas et al. [31], Gholipour et al. [84], Ma et al. [27] and Assaad et al. [107] when applied to the Lorenz time series is listed in Table 5.7.

Iokibe et al. [207] used local fuzzy reconstruction method in short-term prediction of chaotic time series. Martinez-Rego et al. [22] used local dynamic modeling to develop a new forecasting method to predict chaotic time series. The suggested method by Martinez-Rego et al. [22] is consisted of three element of time delay line; information theoretic based clustering method and a set of single layer neural networks. Tao and Xiao [30] and Dhahri and Alimi [208] used a radial basis function network and Garcia and Alarcon [40] employed wavelet networks to forecast chaotic time series.

The prediction accuracy for the 1000 Lorenz time series test samples, for the demonstrated experiments based on feedforward-feedforward configuration and ElamnNARX configuration are compared with the results reported in the literature. Table 5.7 presents the comparison between the prediction errors, MSE, RMSE and NMSE reported in the literature and the proposed method for 1000 Lorenz test samples. These experiments confirm that the proposed method with Elman-NARX, when it is used to forecast the future values of the Lorenz time series, generates better results compared to other prediction methods reported in the literature. 
Table 5.7 Comparison between the prediction errors reported in the literature and the proposed method (1000 Lorenz time series test samples).

\begin{tabular}{|c|c|c|c|c|}
\hline \multirow[b]{2}{*}{ Prediction Method } & \multirow[b]{2}{*}{ Reference } & \multicolumn{3}{|c|}{ Prediction Error } \\
\hline & & $M S E$ & $R M S E$ & NMSE \\
\hline Local Fuzzy Reconstruction & [207] & $2.44 \mathrm{E}-01$ & & \\
\hline Adaptive Neuro-Fuzzy Inference Systems (ANFIS) & {$[145]$} & & $1.43 \mathrm{E}-01$ & \\
\hline Pseudo Gaussian - Radial Basis Function & {$[116]$} & & $9.40 \mathrm{E}-02$ & \\
\hline ARMA - Neural Network & {$[31]$} & & $8.76 \mathrm{E}-02$ & \\
\hline Support Vector Regression & {$[22]$} & & & $1.46 \mathrm{E}-02$ \\
\hline TDL-MLP & {$[22]$} & & & $1.56 \mathrm{E}-04$ \\
\hline DLE-VQIT & {$[22]$} & & & $2.58 \mathrm{E}-04$ \\
\hline RBF with Orthogonal LS & {$[84]$} & & & $1.41 \mathrm{E}-09$ \\
\hline LLNF-LoLiMot & {$[84]$} & & & $9.80 \mathrm{E}-10$ \\
\hline CIFCA-ROLSA & {$[30]$} & $1.30 \mathrm{E}-03$ & & \\
\hline ROLSA & {$[30]$} & 4.63E-02 & & \\
\hline Evolving Recurrent Neural Network (ERNN) & [27] & & & $9.90 \mathrm{E}-10$ \\
\hline Boosted Recurrent Neural Networks (BRNN) & {$[107]$} & & & $3.77 \mathrm{E}-03$ \\
\hline MDE-RBF & {$[208]$} & & 1.70E-01 & \\
\hline Wavelet-Networks B & {$[40]$} & $1.64 \mathrm{E}-02$ & & \\
\hline Proposed Method - Elman-NARX & -- & $1.173 \mathrm{E}-08$ & $1.083 \mathrm{E}-04$ & $1.983 \mathrm{E}-10$ \\
\hline
\end{tabular}

The performance of the proposed forecasting method in this chapter when applied to the sunspot time series is compared with the results published by Sello [82], Gholipour et al. [84], Teo et al. [122], McNish and Lincoln [209], Denkmayr and Cugnon [210], Ma et al. [27] and Koskela et al. [127].

The forecasting method proposed by Sello [82] is based on a local hypothesis of the behaviour on embedding space, employing an optimal number of neighbouring vectors to forecast the future projection. Koskela et al. [127] has compared the 
performance of a multi-layer neural network, finite impulse response (FIR) and an Elman neural network when applied to the sunspot time series prediction.

The error indices, MSE, RMSE and NMSE for the 1000 sunspot time series test samples are compared with the results reported in the literature and presented in Table 5.8. The experiment performed for the sunspot time series confirms that the performance of the proposed method with Elman-NARX configuration is better in prediction of the sunspot time series when compared to other prediction methods reported in the literature.

Table 5.8 Comparison between the prediction errors reported in the literature and the proposed method (1000 sunspot time series test samples).

\begin{tabular}{lllll}
\hline & & \multicolumn{2}{c}{ Prediction Error } \\
\hline Prediction Method & Reference & MSE & RMSE & NMSE \\
\hline Denkmayr & {$[210]$} & & 1.85 \\
Waldmeier & {$[82]$} & & $5.60 \mathrm{E}-01$ \\
Sello- Nonlinear Method & {$[82]$} & & $3.40 \mathrm{E}-01$ \\
WP-MLP (A) & {$[122]$} & & $1.25 \mathrm{E}-01$ \\
Multi-Layer Perceptron (MLP) & {$[127]$} & & $9.79 \mathrm{E}-02$ \\
McNish-Lincoln & {$[209]$} & & $8.00 \mathrm{E}-02$ \\
RBF-OLS & {$[84]$} & & & $4.60 \mathrm{E}-02$ \\
LLNF-LoLiMot & {$[84]$} & & & $3.20 \mathrm{E}-02$ \\
ERNN & {$[27]$} & & $1.29 \mathrm{E}-02$ & $2.80 \mathrm{E}-03$ \\
Proposed Method - Elman-NARX & -- & $1.41 \mathrm{E}-04$ & $1.19 \mathrm{E}-02$ & $5.90 \mathrm{E}-04$ \\
\hline
\end{tabular}

\subsection{Summary}

In this chapter, a new prediction method was developed using a combination of the embedding theorem, residual analysis, ensemble neural networks and genetic algorithms to predict chaotic time series. Based on the embedding theorem, the original time series can 
be unfolded with the embedding dimension and time delay and reconstructed into the phase space points. A neural network was used to predict the future values of the embedded phase space points. In some cases, the residuals of the forecasting method are not random and follow the characteristics of the original time series. The accuracy of the prediction method was further enhanced by analyzing and incorporating the residuals. The residual analysis was repeated several times. Eventually, to capture the relationship between the outcome of neural networks and the original time series, a new neural network was trained. Two configurations for the ensemble neural networks were implemented. In the first configuration, all the selected neural networks were feedforward neural networks. In the second configuration, the combination of Elman and NARX neural networks was utilized.

The developed methods with both feedforward-feedforward and Elman-NARX configurations have been validated for a one-step ahead prediction. The performance of the proposed forecasting methods in terms of error indices has been compared with the performance of reported forecasting methods in the literature. In the current chapter, the neural network architectures and their parameters are determined arbitrarily. In the next chapter, Taguchi's DOE will be implemented to select proper neural network architectures and parameters and improve the performance of the proposed forecasting method.

Despite its simplicity in implementation, the proposed model exhibited a superior performance in predicting the chaotic time series. The developed algorithm was applied to two chaotic systems, Mackey-Glass and Lorenz equations and a real life sunspot time series. It has been concluded that the proposed model can perform more effectively and accurately as compared to other prediction methods for chaotic time series. 


\section{CHAPTER 6}

\section{TAGUCHI'S DESIGN OF EXPERIMENTS IN COMBINATION SELECTION FOR CHAOTIC TIME SERIES FORECASTING METHOD USING ENSEMBLE ARTIFICIAL NEURAL NETWORKS}

Taguchi's design of experiments is an efficient approach to identify the proper factorlevel combinations utilized to improve the result of the proposed forecasting method for chaotic time series. In the proposed method, residual analysis using a combination of embedding theorem and ensemble artificial neural networks is utilized to forecast chaotic time series. Based on the embedding theorem, the embedding parameters are determined and the time series is reconstructed into proper phase space points. The embedded phase space points are fed into the first neural network and trained. The weights and biases are kept to predict the future values of the phase space points and the chaotic time series. The residual of the predicted time series is analyzed, and it was observed that in some events the residuals demonstrate chaotic behaviour. The residuals are considered as a new chaotic time series and reconstructed according to the embedding theorem. A new neural network is trained to predict the future values of the residual time series. The residual analysis can be repeated several times. Finally, the last neural network is trained using the neural networks' result values of the time series and the residuals as input and the original time series as output. The final network is used to capture the relationship 
between the neural networks' result values and the original time series. A systematic approach is introduced using Taguchi's design of experiments to select proper combinations of ensemble artificial neural networks and their parameters.

The method is applied to the Mackey-Glass and the Lorenz equations which produce chaotic time series, and two real life chaotic time series, sunspot and far-infrared NH3 laser time series, to evaluate the performance of the proposed technique. The experimental results confirm that the proposed method can predict the chaotic time series more effectively and accurately when compared with the same method using arbitrary selection of architectures and parameters and other existing forecasting methods in the literature.

The content of the current chapter has been presented at the International Conference on Industrial Engineering and Systems Management [211] and submitted to the journal of Neural Computing and Applications [212].

\subsection{Introduction to Taguchi's Design of Experiments}

In the applications where multiple variables are involved, it is essential to determine the best solution by identifying the proper levels of the variables. Design of experiments (DOE) is a practical tool to investigate the impact on the outcome of a system while changing the variables and to determine the appropriate levels.

The idea of DOE was first introduced by Sir Ronald Fisher in the early 1920s when he proposed a systematic and analytical method to determine the impact of multiple factors on the output of his experiments [213,214]. Factorial experimental design in which experiments are carried out for all factor-level combinations was used by Fisher. 
Taguchi in 1949 developed a method which utilized orthogonal arrays (OA) to reduce the experimental numbers while maintaining reasonably sufficient information $[45,47,50,215,216]$. Taguchi's method gained its reputation mainly in the early $1980 \mathrm{~s}$ [217]. Taguchi's DOE is usually performed with the aid of an analysis of variance (ANOVA). It is one of the most effective techniques for improving the performance of a system by selecting the optimum levels for the involved parameters.

DOE has been utilized by many practitioners for optimization and modelling purposes. The contribution of Taguchi's method to DOE is to simplify the use of this method. This simplification has expanded the applications of DOE.

Many applications of Taguchi's DOE are reported in the literature. Among them, Wang et al. [218] has adopted Taguchi's method to optimize the experimental conditions for the gas chromatographic-mass spectrometric determination. Taguchi method is used by Naidu and Gowda [219] in spray-painting application and Gandhi [220] in the gear sub-assembly of gearboxes. Manna and Bhattacharyya [221] used Taguchi's method to optimize wire EDM parameters for aluminum reinforced metal matrix composite. Taguchi's DOE has helped Sukthomya and Tannock [222] to develop an important manufacturing process for aircraft engines, Mezgar et al. [223] to develop a robust manufacturing system and Tsai and Mort [224] to optimize an integrated manufacturing system.

Taguchi's method has been adopted by many researchers in the field of artificial neural networks (ANN) to find an optimum design for the networks. Kim and Yum [43], Khaw et al. [44], Lin and Tseng [45], Ortiz-Rodríguez et al. [46], Packianather and Drake [47], Wang et al. [48], Yang and Lee [49] and Peterson et al. [50] have used 
Taguchi's DOE to optimize the design of ANN. Mouli et al. [51] has demonstrated that Taguchi's method can be utilized as a pre-step to the neural network method to obtain the optimum result.

In the current research work, Taguchi's design of experiments is utilized as a prior step to determine the factor-level combinations to improve the performance of the proposed method to forecast chaotic time series.

\subsection{Taguchi's Design of Experiments Method}

Taguchi's design of experiments is an efficient method to identify the optimum factor-level combinations to minimize variation while maintaining the mean at the desired level. Taguchi's DOE can improve the performance of a process or a system with a limited number of experimental data based on orthogonal arrays (OA). OA is the matrix of combinations with the shortest possible elements. Similar to DOE, the purpose of Taguchi's method is to study the impact on the performance of the system while all the design parameters are simultaneously varied. The two important elements in selecting the orthogonal arrays are the factors and interactions and the levels for each factor [51].

The standard orthogonal arrays established by Taguchi contain 18 basic OA. The name of the OA indicates the number of rows, columns and the number of levels in each column. For instance, orthogonal array L27 $\left(3^{10}\right)$ consists of 27 rows and 10 columns and each column has 3 levels. When an appropriate OA is selected, a table of combinations will be formed and the experiments will be performed for each row of the OA table. The results of the experiments will be analyzed using the analysis of variance (ANOVA) method. Based on Taguchi's approach, the ratio of signal (target or the mean of the 
outcomes) to the noise (square deviation of the outcomes) which is called the $\mathrm{S} / \mathrm{N}$ ratio can be calculated by Eq. (36):

$S / N=-10 \log _{10}\left[\frac{1}{n} \sum_{n} \frac{1}{z_{i}^{2}}\right]$

where $n$ is the number of variables and $z_{i}$ is the value of each variable. For each level of each parameter, the $S / N$ ratios are calculated and the graphs are generated. The proper level for each parameter is achieved based on the highest $S / N$ ratio [51].

\subsection{Implementation of Taguchi's DOE to the Forecasting Method}

The process of forecasting the future values of a time series using residual analysis and the ensemble method involves a number of parameters including the neural network architecture, the neural network transfer function, the training method and others, which affect the end result. In this study, Taguchi's design of experiments is employed to improve the proposed forecasting method by selecting the factors and their levels, positioning them appropriately into a suitable OA, and conducting experiments. The factors are selected from neural network architectures: FFNN, Elman and NARX, MATLAB [191] transfer functions: purelin and tansig and MATLAB [191] training methods: traingdx, trainbr and trainlm. Weights and biases of the neural networks which cause variation in the result of the forecasting method are considered as noise factors. Each experiment is taken from the OA and the performance of the forecasting method is measured for that trial. The performance of the forecasting method is determined by the normalized mean square error (NMSE) of the real data and the forecast values. The resulting performances for all the experiments are analyzed and the appropriate 
combination setting which delivers the best performance is identified. In this work, eight factors are identified as control factors and evaluated at two levels as shown in Table 6.1.

In the classical experimentation approach of identifying the critical parameters and finding the optimum values for achieving an optimum solution, a full factorial matrix (in this case $2^{8}$ or 256 experiments) would have been required. This would involve holding all parameters constant while changing one parameter during the experiment. The process would be repeated until an optimal condition was realized. With respect to time and budget considerations, the full factorial matrix setup and experimentation is very costly and practically un-doable due to time constraints.

Table 6.1 The control factors and their associated levels.

\begin{tabular}{lllll}
\hline No. & Factors & Abbreviation & Level 1 & Level 2 \\
\hline 1 & $1^{\text {st }}, 2^{\text {nd }} \& 3^{\text {rd }}$ Tier Neural Network Architecture & $123 \mathrm{NT}$ & FFNN & Elman \\
2 & Final Neural Network Architecture & $4 \mathrm{NT}$ & FFNN & NARX \\
3 & $1^{\text {st }}, 2^{\text {nd }} \& 3^{\text {rd }}$ Tier Network Hidden Transfer Function & $123 \mathrm{HTF}$ & tansig & purelin \\
4 & Final Network Hidden Transfer Function & $4 \mathrm{HTF}$ & tansig & purelin \\
5 & $1^{\text {st }}, 2^{\text {nd }} \& 3^{\text {rd }}$ Tier Network Output Transfer Function & $123 \mathrm{OTF}$ & tansig & purelin \\
6 & Final Network Output Transfer Function & $4 \mathrm{OTF}$ & tansig & purelin \\
7 & $1^{\text {st }}, 2^{\text {nd }} \& 3^{\text {rd }}$ Tier Network Training Method & $123 \mathrm{TM}$ & traingdx & trainbr \\
8 & Final Network Training Method & $4 \mathrm{TM}$ & trainbr & trainlm \\
\hline
\end{tabular}

Taguchi's design of experiments offers a shorter matrix of experimental design based on the Latin square concept of orthogonal arrays. For this study, using the Taguchi method concept, only 16 experiments (L16 orthogonal array) were required. From the measured output performance (NMSE between forecast data and real data) of the selected 16 orthogonal array experimentation set, all critical parameters and their values were identified. These parameters and values were used to improve the output of the proposed 
forecasting method. To allow noise factors to be represented in the results, two replications of each experiment were used. The Taguchi L16 orthogonal array matrix is shown in Table 6.2 .

Four tiers are selected to analyze and forecast the time series. In the first tier, a neural network is trained to forecast the future values of the original time series. In the second tier, a new neural network is trained to forecast the residuals of the first tier. In the third tier, the residuals of the second tier are trained by a new neural network. Finally, in the last tier, a neural network is trained using the neural networks' result values of the time series and the residuals as input and the original time series as output. The final network is used to capture the relationship between the neural networks' result values and the original time series. The neural network architectures, hidden layer transfer functions, output transfer functions and training functions are selected according to Table 6.2. Two independent trials of each row of Table 6.2 are performed. Each experimental trial is made up of 16 experiments as described in Table 6.2. Therefore, for the two independent trials, a total of 32 runs are performed.

In Taguchi's DOE, the weights and biases of the neural network are considered as noise and the intent is to minimize the NMSE between forecast data and real data. From the results generated by the Minitab software package [225], each factor's contribution to the obtained outputs is determined. In this study, the focus is on the mean value rather than on the signal-to-noise $(\mathrm{S} / \mathrm{N})$ value. In other words, it is more desirable to improve the error between forecast data and real data rather than improving the output variance of the forecasting process. Finally, an analysis of variance (ANOVA) is carried out based on 
the prepared experiments and their output results. A confidence level of 95 percent was selected to evaluate the importance of each factor.

Table 6.2 Taguchi's DOE setting; orthogonal array L16 $\left(2^{8}\right)$.

\begin{tabular}{|c|c|c|c|c|c|c|c|c|}
\hline No. & $123 N T$ & $4 N T$ & $123 H T F$ & $4 H T F$ & $1230 T F$ & $4 O T F$ & $123 T M$ & $4 T M$ \\
\hline 1 & $\mathrm{FF}$ & $\mathrm{FF}$ & tansig & tansig & tansig & tansig & traingdx & trainbr \\
\hline 2 & $\mathrm{FF}$ & $\mathrm{FF}$ & tansig & purelin & tansig & purelin & trainbr & trainlm \\
\hline 3 & $\mathrm{FF}$ & $\mathrm{FF}$ & purelin & tansig & purelin & tansig & trainbr & trainlm \\
\hline 4 & $\mathrm{FF}$ & $\mathrm{FF}$ & purelin & purelin & purelin & purelin & traingdx & trainbr \\
\hline 5 & $\mathrm{FF}$ & NARX & tansig & tansig & purelin & purelin & traingdx & trainlm \\
\hline 6 & $\mathrm{FF}$ & NARX & tansig & purelin & purelin & tansig & trainbr & trainbr \\
\hline 7 & $\mathrm{FF}$ & NARX & purelin & tansig & tansig & purelin & trainbr & trainbr \\
\hline 8 & $\mathrm{FF}$ & NARX & purelin & purelin & tansig & tansig & traingdx & trainlm \\
\hline 9 & Elman & $\mathrm{FF}$ & tansig & tansig & purelin & purelin & trainbr & trainbr \\
\hline 10 & Elman & $\mathrm{FF}$ & tansig & purelin & purelin & tansig & traingdx & trainlm \\
\hline 11 & Elman & $\mathrm{FF}$ & purelin & tansig & tansig & purelin & traingdx & trainlm \\
\hline 12 & Elman & $\mathrm{FF}$ & purelin & purelin & tansig & tansig & trainbr & trainbr \\
\hline 13 & Elman & NARX & tansig & tansig & tansig & tansig & trainbr & trainlm \\
\hline 14 & Elman & NARX & tansig & purelin & tansig & purelin & traingdx & trainbr \\
\hline 15 & Elman & NARX & purelin & tansig & purelin & tansig & traingdx & trainbr \\
\hline 16 & Elman & NARX & purelin & purelin & purelin & purelin & trainbr & trainlm \\
\hline
\end{tabular}

\subsection{Numerical Analysis Using Taguchi's DOE Method}

In order to evaluate the proposed method, the developed technique is applied to two chaotic systems, Mackey-Glass and Lorenz equations and two real life chaotic time series, sunspot and far-infrared time series. Later, the performance of the forecasting method configured by the appropriate factor-level combinations determined by Taguchi's design of 
experiment is compared with the results of the same forecasting method with arbitrary selection of architectures and parameters and the results reported in the literature.

\subsubsection{Lorenz Equations}

The details of the Lorenz equations have been presented in Section 5.2.2. Similar to Section 5.2.2 the parameters in Eq. (35) are selected according to the previous reports $[27,84]$. The $x$-coordinate of the Lorenz time series is considered for prediction and a time series with a length of 2500 is generated as described in the research work by Ma et al. [27]. The first 1500 samples were used as training data, while the remaining 1000 were used to test the proposed model.

\subsubsection{Mackey-Glass Equation}

The details of the Mackey-Glass equation have been presented in Section 4.2.1. Similar to Section 4.2.1, the parameters in Eq. (26) are selected according to the previous report by Zhang et al. [66], where the constants are taken to be $a=0.2, b=0.1$ and $c=10$ and chaotic time series is generated by $\tau=17$ and initial value $x(0)=1.2$. A chaotic time series data set with length of 1000 is generated by Eq. (26). The first 500 data were used as training data, while the remaining 500 were used to test the proposed model.

\subsubsection{Sunspot Time Series}

The details of the sunspot time series have been presented in Section 5.3.3. The monthly smoothed sunspot time series has been obtained from the SIDC (World Data Center for the Sunspot Index) [204]. To compare the results with some of the studies 
published in the literature, data are selected in the same conditions reported by Ma et al. [27] and Gholipour et al. [84]. The sunspot series from November 1834 to June 2001 (2000 points) are selected and scaled between [0,1]. The first 1000 samples of the time series are selected for training and the remaining 1000 samples are kept to test the prediction method.

\subsubsection{Far-Infrared NH3 Laser Time Series}

The far-infrared NH3 Laser time series is set A from the Santa Fe time series forecasting competition [226,227]. It is a real world data set recorded from the farinfrared NH3 laser in a chaotic state. The training set is a series of 1000 values and the goal is to apply the proposed forecasting method in this study to forecast one step ahead for the next 100 data.

\subsubsection{Numerical Experiments}

The embedding parameters for the Lorenz time series $D=3$ and $T=3$, the Mackey-Glass time series $D=3$ and $T=7$, the sunspot time series $D=5$ and $T=1$ and the far-infrared NH3 laser time series $D=7$ and $T=2$ are estimated using TSTOOL [190] and accordingly phase space points are reconstructed.

For all the time series, the embedding dimensions $\left(D_{1} \& D_{2}\right)$ and time delays $\left(T_{1}\right.$ $\& T_{2}$ ) for the second and third tiers are selected based on the characteristics of the residuals. The neural network architectures and the remaining parameters are selected according to Table 6.2. Table 6.3 presents the performance of the forecasting method (NMSE) for each experiment for all four time series. 
Table 6.3 Taguchi's DOE setting; orthogonal array L16 $\left(2^{8}\right)$.

\begin{tabular}{lllllllll}
\hline & \multicolumn{3}{c}{ Lorenz } & \multicolumn{2}{c}{ Mackey-Glass } & \multicolumn{2}{c}{ Sunspot } & \multicolumn{2}{c}{ NH3 Laser } \\
\hline No. & RUN1 & RUN2 & RUN1 & RUN2 & RUN1 & RUN2 & RUN1 & RUN2 \\
\hline 1 & 2.9549 & 2.9502 & 2.8145 & 2.8223 & 1.6482 & 1.6487 & $8.27 \mathrm{E}-01$ & $8.28 \mathrm{E}-01$ \\
2 & 2.7552 & 2.7551 & 2.9158 & 2.916 & 1.6578 & 1.6612 & $8.05 \mathrm{E}-01$ & $8.05 \mathrm{E}-01$ \\
3 & 2.853 & 2.848 & 2.9606 & 2.9608 & 1.6323 & 1.6321 & $8.15 \mathrm{E}-01$ & $8.14 \mathrm{E}-01$ \\
4 & 2.953 & 2.9533 & 2.7606 & 2.7704 & 1.6242 & 1.6243 & $8.25 \mathrm{E}-01$ & $8.25 \mathrm{E}-01$ \\
5 & $3.00 \mathrm{E}-05$ & $3.00 \mathrm{E}-05$ & $8.80 \mathrm{E}-04$ & $8.79 \mathrm{E}-04$ & $3.89 \mathrm{E}-04$ & $3.84 \mathrm{E}-04$ & $8.04 \mathrm{E}-02$ & $8.03 \mathrm{E}-02$ \\
6 & $9.55 \mathrm{E}-01$ & $9.56 \mathrm{E}-01$ & $3.38 \mathrm{E}-01$ & $3.38 \mathrm{E}-01$ & $7.10 \mathrm{E}-01$ & $7.09 \mathrm{E}-01$ & $5.81 \mathrm{E}-01$ & $5.82 \mathrm{E}-01$ \\
7 & $8.13 \mathrm{E}-01$ & $8.13 \mathrm{E}-01$ & $4.22 \mathrm{E}-01$ & $4.28 \mathrm{E}-01$ & $9.03 \mathrm{E}-02$ & $9.11 \mathrm{E}-02$ & $5.03 \mathrm{E}-01$ & $5.04 \mathrm{E}-01$ \\
8 & $6.30 \mathrm{E}-05$ & $6.29 \mathrm{E}-05$ & $4.22 \mathrm{E}-05$ & $4.21 \mathrm{E}-05$ & $6.03 \mathrm{E}-03$ & $6.03 \mathrm{E}-03$ & $6.03 \mathrm{E}-02$ & $6.03 \mathrm{E}-02$ \\
9 & 2.01643 & 2.01642 & 2.6813 & 2.6797 & 1.6094 & 1.6091 & $8.20 \mathrm{E}-01$ & $8.19 \mathrm{E}-01$ \\
10 & $7.11 \mathrm{E}-01$ & $7.12 \mathrm{E}-01$ & 1.91116 & 1.91217 & 1.0056 & 1.0043 & $3.20 \mathrm{E}-01$ & $3.21 \mathrm{E}-01$ \\
11 & $6.64 \mathrm{E}-01$ & $6.55 \mathrm{E}-01$ & 1.98107 & 1.9811 & 1.1314 & 1.1317 & $4.88 \mathrm{E}-01$ & $4.87 \mathrm{E}-01$ \\
12 & 2.2823 & 2.2832 & 2.5983 & 2.5982 & 1.5314 & 1.5316 & $8.88 \mathrm{E}-01$ & $8.86 \mathrm{E}-01$ \\
13 & $2.31 \mathrm{E}-09$ & $2.30 \mathrm{E}-09$ & $4.37 \mathrm{E}-06$ & $4.29 \mathrm{E}-06$ & $2.55 \mathrm{E}-03$ & $2.56 \mathrm{E}-03$ & $9.26 \mathrm{E}-03$ & $9.28 \mathrm{E}-03$ \\
14 & $3.20 \mathrm{E}-09$ & $3.22 \mathrm{E}-09$ & $5.72 \mathrm{E}-05$ & $5.73 \mathrm{E}-05$ & $5.63 \mathrm{E}-04$ & $5.60 \mathrm{E}-04$ & $7.73 \mathrm{E}-03$ & $7.69 \mathrm{E}-03$ \\
15 & $3.31 \mathrm{E}-08$ & $3.31 \mathrm{E}-08$ & $9.2 \mathrm{E}-06$ & $9.21 \mathrm{E}-06$ & $5.60 \mathrm{E}-05$ & $5.61 \mathrm{E}-05$ & $4.30 \mathrm{E}-02$ & $4.31 \mathrm{E}-02$ \\
16 & $1.20 \mathrm{E}-10$ & $1.19 \mathrm{E}-10$ & $5.62 \mathrm{E}-07$ & $5.62 \mathrm{E}-07$ & $5.59 \mathrm{E}-04$ & $5.59 \mathrm{E}-04$ & $8.30 \mathrm{E}-03$ & $8.32 \mathrm{E}-03$ \\
\hline
\end{tabular}

The results of the ANOVA at 95\% confidence level are shown in Table 6.4. Each column in Table 6.4 contains information regarding the determination of the significance of each factor to the response (NMSE). The factor with the largest $F$ value has the greatest impact and based on that, the ranking of each factor is determined as shown in the response Table 6.5 [225]. For all four time series, the $F$ Test at 95\% level of confidence indicated that Tier Neural Network Architecture, Final Neural Network 
Architecture, Tier Network Training Method and Final Network Training Method among other factors are significant because their $p$-values are less than 0.05 .

Table 6.4 The result of ANOVA analysis.

\begin{tabular}{lllllllll}
\hline & \multicolumn{2}{c}{ Lorenz } & \multicolumn{3}{c}{ Mackey-Glass } & \multicolumn{2}{c}{ Sunspot } & \multicolumn{2}{c}{ NH3 Laser } \\
\hline Factors & $F$ & $P$ & $F$ & $P$ & $F$ & $P$ & $F$ & $P$ \\
\hline 123NT & 20.48 & 0.003 & 19.63 & 0.003 & 11.86 & 0.011 & 58.66 & 0.000 \\
4NT & 84 & 0.000 & 831.52 & 0.000 & 330.32 & 0.000 & 322.93 & 0.000 \\
123HTF & 0.01 & 0.923 & 0.01 & 0.927 & 1.04 & 0.342 & 0.51 & 0.500 \\
4HTF & 0.05 & 0.834 & 0.24 & 0.64 & 0.48 & 0.51 & 0.13 & 0.730 \\
123OTF & 0.0000 & 0.989 & 0.01 & 0.909 & 0.71 & 0.428 & 0.14 & 0.715 \\
4OTF & 0.11 & 0.751 & 0.04 & 0.843 & 0.48 & 0.513 & 0.000 & 0.979 \\
123TM & 6.83 & 0.035 & 12.55 & 0.009 & 8.99 & 0.020 & 50.51 & 0.000 \\
4TM & 8.83 & 0.021 & 7.25 & 0.031 & 8.57 & 0.022 & 58.31 & 0.000 \\
\hline
\end{tabular}

The response Table 6.5 shows the average of each response for each level of each factor. The table of responses determines the rank of each factor based on Delta statistics, which compares the relative magnitude of effects. The ranks are assigned according to the Delta values with the highest Delta value being the first rank [225]. Therefore, based on the response Table 6.5, Final Neural Network Architecture and Tier Neural Network Architecture have the greatest influence for all four time series. The Tier Network Training Method and the Final Network Training Method have the next influence for the Mackey-Glass and sunspot time series, respectively, and with the change in the order; Final Network Training and Tier Network Training have the next influence for the Lorenz and far-infrared NH3 laser time series, respectively. 
Table 6.5 The response table for Lorenz time series, Mackey-Glass time series, sunspot time series and far-infrared NH3 laser time series.

\begin{tabular}{llllllllll}
\hline Time Series & Level & $123 N T$ & $4 N T$ & $123 H T F$ & $4 H T F$ & $123 O T F$ & 4 OTF & $123 T M$ & $4 T M$ \\
\hline \multirow{4}{*}{ Lorenz } & 1 & 0.7088 & 2.1478 & 1.1949 & 1.2073 & 1.1859 & 1.1497 & 1.4592 & 1.4967 \\
& 2 & 1.66 & 0.2211 & 1.174 & 1.1616 & 1.1829 & 1.2192 & 0.9097 & 0.8722 \\
& Delta & 0.9512 & 1.9266 & 0.021 & 0.0458 & 0.003 & 0.0694 & 0.5496 & 0.6246 \\
& Rank & 2 & 1 & 7 & 6 & 8 & 5 & 4 & 3 \\
\hline \multirow{3}{*}{ Mackey-Glass } & 1 & 1.14645 & 2.579 & 1.34132 & 1.31618 & 1.33216 & 1.34612 & 1.48979 & 1.45321 \\
& 2 & 1.52805 & 0.0955 & 1.33318 & 1.35832 & 1.34234 & 1.32838 & 1.1847 & 1.22128 \\
& Delta & 0.38161 & 2.4835 & 0.00815 & 0.04215 & 0.01019 & 0.01773 & 0.30509 & 0.23193 \\
& Rank & 2 & 1 & 8 & 5 & 7 & 6 & 3 & 4 \\
\hline \multirow{5}{*}{ Sunspot } & 1 & 0.6601 & 1.4802 & 0.7521 & 0.8171 & 0.8226 & 0.7646 & 0.9045 & 0.9018 \\
& 2 & 0.9214 & 0.1013 & 0.8294 & 0.7644 & 0.7589 & 0.8169 & 0.677 & 0.6797 \\
& Delta & 0.2613 & 1.3789 & 0.0773 & 0.0527 & 0.0638 & 0.0523 & 0.2274 & 0.2221 \\
& Rank & 2 & 1 & 5 & 7 & 6 & 8 & 3 & 4 \\
\hline NH3 Laser & 1 & 0.3229 & 0.7233 & 0.4536 & 0.4369 & 0.4366 & 0.4421 & 0.5536 & 0.5618 \\
& 2 & 0.5622 & 0.1617 & 0.4314 & 0.4481 & 0.4485 & 0.443 & 0.3315 & 0.3232 \\
& Delta & 0.2394 & 0.5616 & 0.0222 & 0.0112 & 0.0119 & 0.0008 & 0.2221 & 0.2386 \\
& Rank & 2 & 1 & 5 & 7 & 6 & 8 & 4 & 3 \\
\hline
\end{tabular}

\subsection{Comparing the Results}

The purpose of this study is to improve the performance of the developed forecasting method by reducing NMSE; therefore, the factor-levels which produce the lowest mean are desirable. The level averages in the response Table 6.5 show that the means are minimized when the Tier Neural Network Architecture is Elman, Final Neural Network Architecture is NARX, the Tier Network Training Method is traingdx and the Final Network Training Method is trainlm. The remaining factors do not show any significance. 
The proper levels which are obtained from Taguchi's DOE are used in the prediction of the four selected time series. In order to evaluate the forecasting performance and compare this with the results reported in the literature, the mean squared error (MSE), normalized mean squared error (NMSE) and root mean squared error (RMSE) are calculated.

The prediction accuracy of the proposed method when the parameters are selected arbitrarily or with Taguchi's DOE is compared to the prediction performance of the methods presented in the literature for the Lorenz time series. The performance of the forecasting methods by Jang et al. [145], Rojas et al. [116], Rojas et al. [31], Gholipour et al. [84], Ma et al. [27] and Assaad et al. [107], Iokibe et al. [207], Martinez-Rego et al. [22], Tao and Xiao [30], Dhahri and Alimi [208] and Garcia and Alarcon [40] when applied to the Lorenz time series is listed in Table 6.6. Figure 6.1 compares the original values of the Lorenz time series with their predicted values.

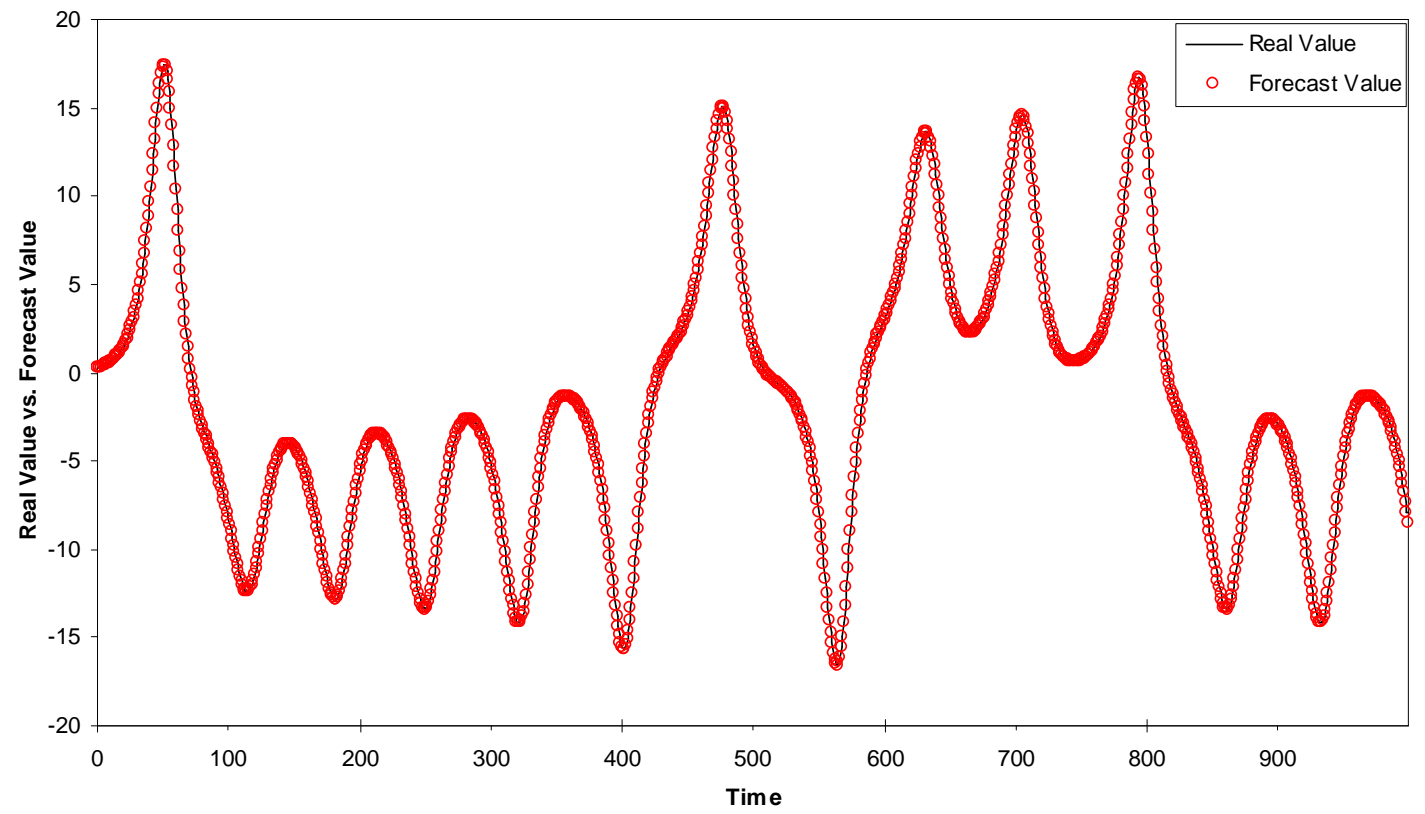

Figure 6.1 The comparison between original Lorenz time series and the final predicted values. 
Table 6.6 presents the comparison between the prediction errors, MSE, RMSE and NMSE reported in the literature and the proposed method for 1000 Lorenz test data. This experiment confirms the superiority of the proposed method in the prediction of chaotic time series when it is compared with other prediction methods reported in the literature.

Table 6.6 Comparison between the prediction errors reported in the literature and the proposed method (1000 Lorenz time series validation data).

\begin{tabular}{|c|c|c|c|c|}
\hline \multirow[b]{2}{*}{ Prediction Method } & \multirow[b]{2}{*}{ Reference } & \multicolumn{3}{|c|}{ Prediction Error } \\
\hline & & $M S E$ & $R M S E$ & NMSE \\
\hline Local Fuzzy Reconstruction & [207] & 2.44E-01 & & \\
\hline ANFIS & {$[145]$} & & $1.43 \mathrm{E}-01$ & \\
\hline Pseudo Gaussian-Radial Basis Functio & {$[116]$} & & $9.40 \mathrm{E}-02$ & \\
\hline ARMA - Neural Network & {$[31]$} & & $8.76 \mathrm{E}-02$ & \\
\hline Support Vector Regression & {$[22]$} & & & $1.46 \mathrm{E}-02$ \\
\hline TDL-MLP & {$[22]$} & & & $1.56 \mathrm{E}-04$ \\
\hline DLE-VQIT & {$[22]$} & & & $2.58 \mathrm{E}-04$ \\
\hline RBF with OLS & {$[84]$} & & & $1.41 \mathrm{E}-09$ \\
\hline LLNF-LoLiMot & {$[84]$} & & & $9.80 \mathrm{E}-10$ \\
\hline CIFCA-ROLSA & {$[30]$} & $1.30 \mathrm{E}-03$ & & \\
\hline ROLSA & {$[30]$} & 4.63E-02 & & \\
\hline Evolving Recurrent Neural Network & {$[27]$} & & & $9.90 \mathrm{E}-10$ \\
\hline Boosted Recurrent Neural Networks & [107] & & & 3.77E-03 \\
\hline MDE-RBF & [208] & & $1.70 \mathrm{E}-01$ & \\
\hline Wavelet-Networks B & {$[40]$} & $1.64 \mathrm{E}-02$ & & \\
\hline Proposed Method - Taguchi's DOE & & 1.95E-09 & $4.41 \mathrm{E}-05$ & 3.29E-11 \\
\hline
\end{tabular}

Figure 6.2 compares the original values of the Mackey-Glass time series with their predicted values. Table 6.7 presents the comparison between the prediction errors, 
MSE, RMSE and NMSE, reported in Wang \& Mendel [205], Cho \& Wang [115], Jang et al. [145] and Zhang et al. [39], Rojas et al. [116], Rojas et al. [31], Kim \& Kim [154], Gholipour et al. [84], Teo et al. [122], Zhang and Man [1], Assaad et al. [107], Inoue et al. [206] and the proposed method for 500 Mackey-Glass test data. The results presented in Table 6.7 show the superiority of the proposed method in predicting chaotic time series when compared with other prediction methods reported in the literature.

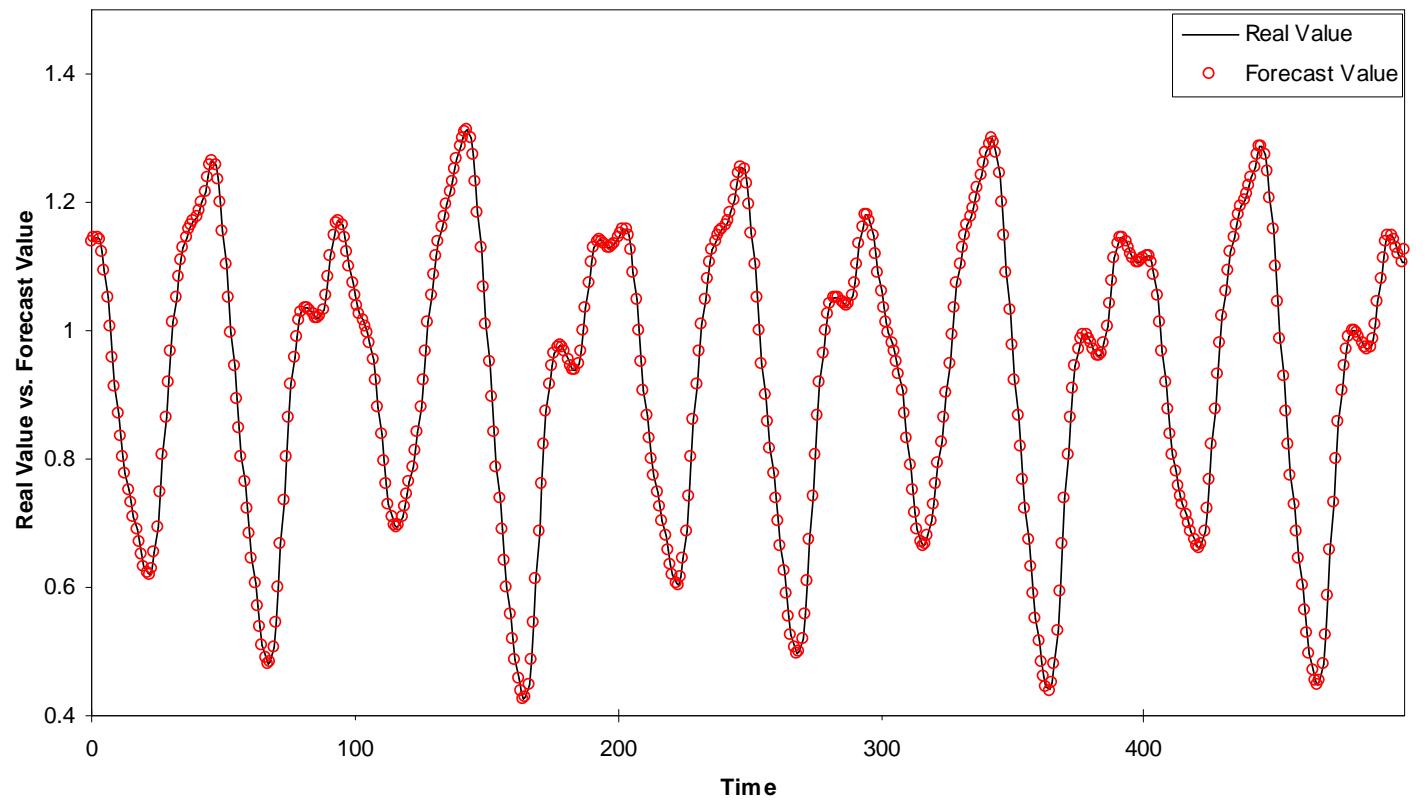

Figure 6.2 Comparison between original Mackey-Glass time series and the final predicted values. 
Table 6.7 Comparison between the prediction errors reported in the literature and the proposed method (500 Mackey-Glass time series validation data).

\begin{tabular}{|c|c|c|c|c|}
\hline \multirow[b]{2}{*}{ Prediction Method } & \multirow[b]{2}{*}{ Reference } & \multicolumn{3}{|c|}{ Prediction Error } \\
\hline & & $M S E$ & $R M S E$ & NMSE \\
\hline Hybrid Neural Network (HNN) & [206] & & & $5.30 \mathrm{E}-02$ \\
\hline Neuro-Fuzzy System with Delay & [39] & & & $1.26 \mathrm{E}-03$ \\
\hline BRNN & [107] & & & $1.60 \mathrm{E}-04$ \\
\hline $\mathrm{RNN}-\mathrm{D}=3$ & {$[1]$} & 4.15E-04 & & \\
\hline RNN - Multi-Dimension & {$[1]$} & $3.81 \mathrm{E}-04$ & & \\
\hline WP-MLP (A) & {$[122]$} & $1.25 \mathrm{E}-06$ & & \\
\hline Auto-Regressive Model & {$[31]$} & & $1.90 \mathrm{E}-01$ & \\
\hline Product T-Norm & [205] & & $9.10 \mathrm{E}-02$ & $2.70 \mathrm{E}-08$ \\
\hline Min T-Norm & [205] & & $9.00 \mathrm{E}-02$ & \\
\hline GA and Fuzzy System - 9 MFs & {$[154]$} & & $3.80 \mathrm{E}-02$ & \\
\hline RBF - Adaptive Fuzzy System I & [115] & & $1.14 \mathrm{E}-02$ & \\
\hline ANFIS and Fuzzy System & {$[145]$} & & $7.00 \mathrm{E}-03$ & \\
\hline Pseudo Gaussian - Radial Basis Function & [116] & & $2.80 \mathrm{E}-03$ & \\
\hline ARMA - Neural Network & {$[31]$} & & $2.50 \mathrm{E}-03$ & \\
\hline ANFIS & {$[84]$} & & $1.50 \mathrm{E}-03$ & \\
\hline RBF-OLS & {$[84]$} & & $1.02 \mathrm{E}-03$ & \\
\hline LLNF-LoLiMot & {$[84]$} & & $9.61 \mathrm{E}-04$ & \\
\hline ERNN & {$[27]$} & & $4.20 \mathrm{E}-05$ & $3.15 \mathrm{E}-08$ \\
\hline The Proposed Method - Taguchi's DOE & & $8.19 \mathrm{E}-10$ & $2.87 \mathrm{E}-05$ & $1.59 \mathrm{E}-08$ \\
\hline
\end{tabular}

Figure 6.3 compares the original values of the sunspot time series with their predicted values. Figure 6.4 shows the marked area in Figure 6.3. The error indices, MSE, RMSE and NMSE for the 1000 sunspot time series test data are compared with the results reported in Sello [82], Gholipour et al. [84], Teo et al. [122], McNish and Lincoln 
[209], Denkmayr and Cugnon [210], Ma et al. [27] and Koskela et al. [25], Koskela et al. [127] and presented in Table 6.8. According to Table 6.8, the performance of the proposed method supersedes the performance of other methods in the literature.

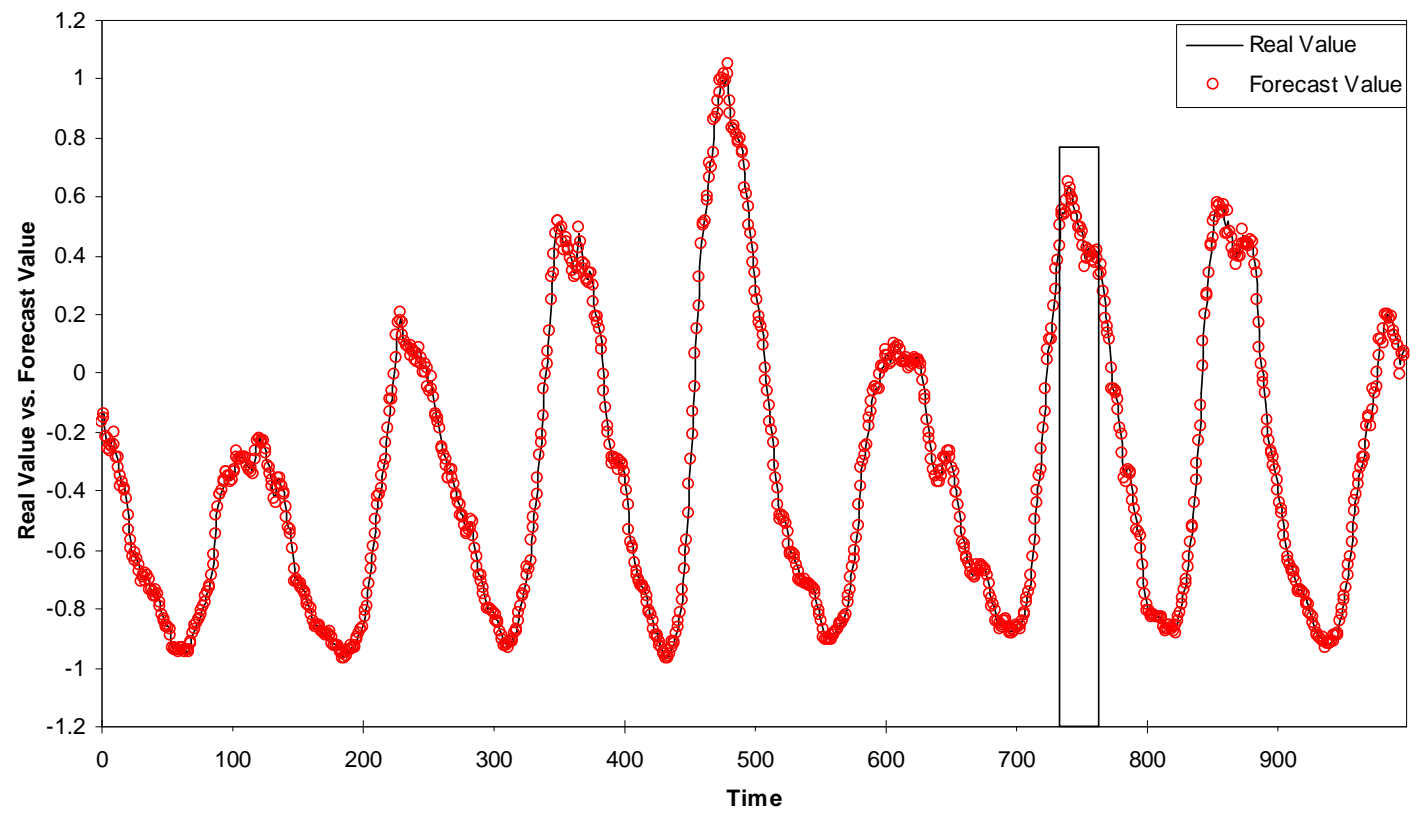

Figure 6.3 The comparison between original sunspot time series and the final predicted values.

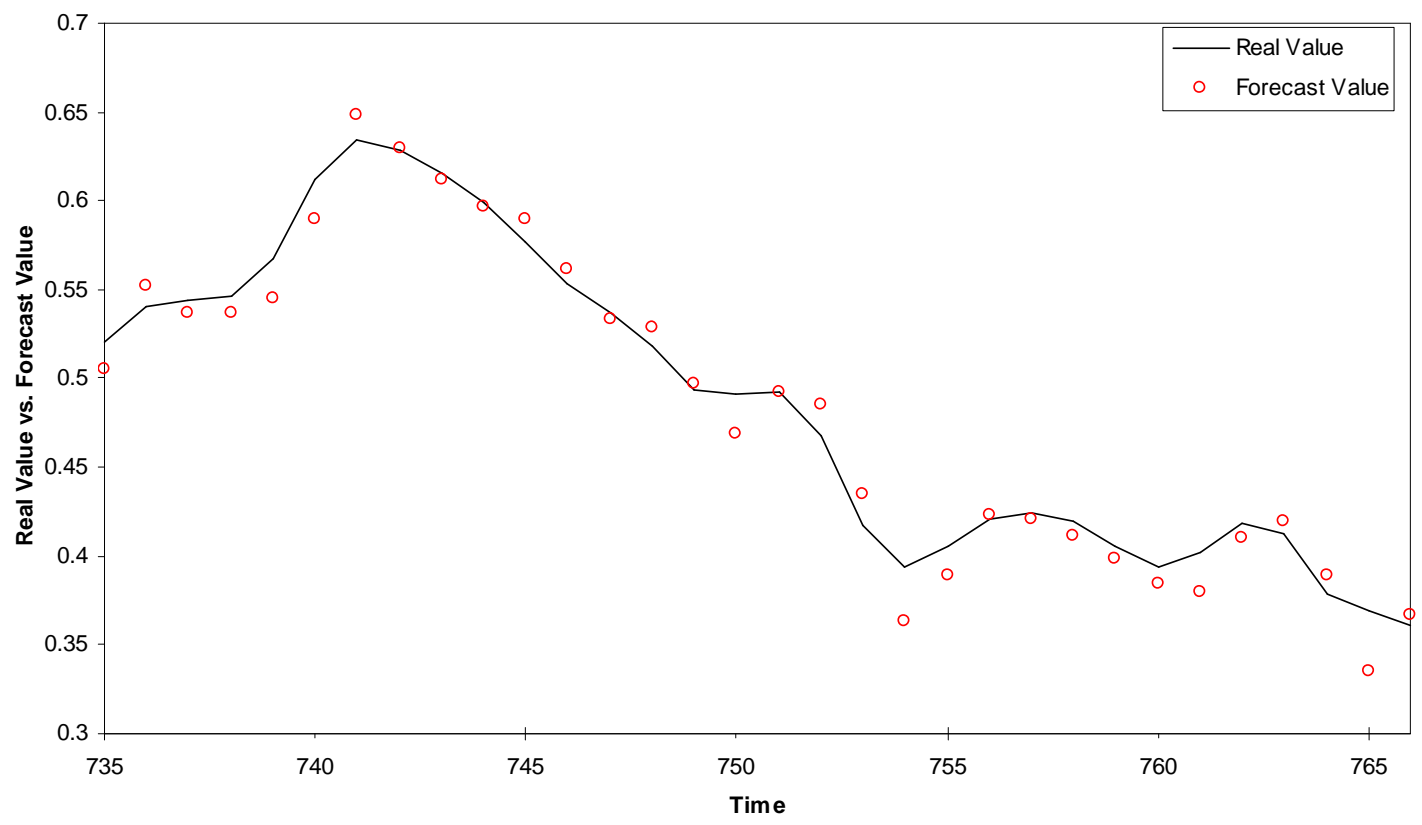

Figure 6.4 The marked area in Figure 6.3. 
Table 6.8 Comparison between the prediction errors reported in the literature and the proposed method (1000 sunspot time series validation data).

\begin{tabular}{|c|c|c|c|c|}
\hline \multirow[b]{2}{*}{ Prediction Method } & \multirow[b]{2}{*}{ Reference } & \multicolumn{3}{|c|}{ Prediction Error } \\
\hline & & $M S E$ & RMSE & $N M S E$ \\
\hline WP-MLP (A) & [122] & & & $1.25 \mathrm{E}-01$ \\
\hline McNish-Lincoln & [209] & & & $8.00 \mathrm{E}-02$ \\
\hline Sello - Nonlinear Method & {$[82]$} & & & $3.40 \mathrm{E}-01$ \\
\hline Waldmeier & {$[82]$} & & & $5.60 \mathrm{E}-01$ \\
\hline Denkmayr & [210] & & & 1.85 \\
\hline RBF-OLS & [84] & & & 4.60E-02 \\
\hline LLNF-LoLiMot & [84] & & & $3.20 \mathrm{E}-02$ \\
\hline ERNN & {$[27]$} & & $1.29 \mathrm{E}-02$ & $2.80 \mathrm{E}-03$ \\
\hline Multi-Layer Perceptron (MLP) & [127] & & & 9.79E-02 \\
\hline \multicolumn{2}{|c|}{ The Proposed Method - Taguchi's DOE } & $1.202 \mathrm{E}-04$ & $1.10 \mathrm{E}-02$ & $5.038 \mathrm{E}-04$ \\
\hline
\end{tabular}

Figure 6.5 compares the original values of the far-infrared NH3 laser time series with their predicted values using the proposed method in this chapter. The error index, NMSE for 100 far-infrared NH3 laser time series test data for the demonstrated experiment is compared with the results published in the literature.

Wan [126] proposed a forecasting method based on the finite impulse response (FIR) method. Koskela et al. [127] utilized a recurrent self-organizing map (RSOM) with local linear models to forecast chaotic time series. Dorffner et al. [228] developed a forecasting method based on a recurrent neural network (RNN) architecture called long short-term memory (LSTM). Assaad et al. [107] proposed a forecasting method based on boosted recurrent neural networks. Zheng et al. [137] implemented a multi-scale support 
vector regression (MS-SVR) method which has several different kernel scales in prediction of chaotic time series. Menezes et al. [28] applied a NARX neural network to chaotic time series forecasting and compared the achieved results with the results obtained from time delay neural networks (TDNN) and an Elman neural network. Table 6.9 presents the comparison between the prediction errors, NMSE, reported in the literature and the proposed method for 100 far-infrared NH3 laser test data. This experiment also confirms the superiority of the proposed method in predicting chaotic time series when compared with other prediction methods reported in the literature.

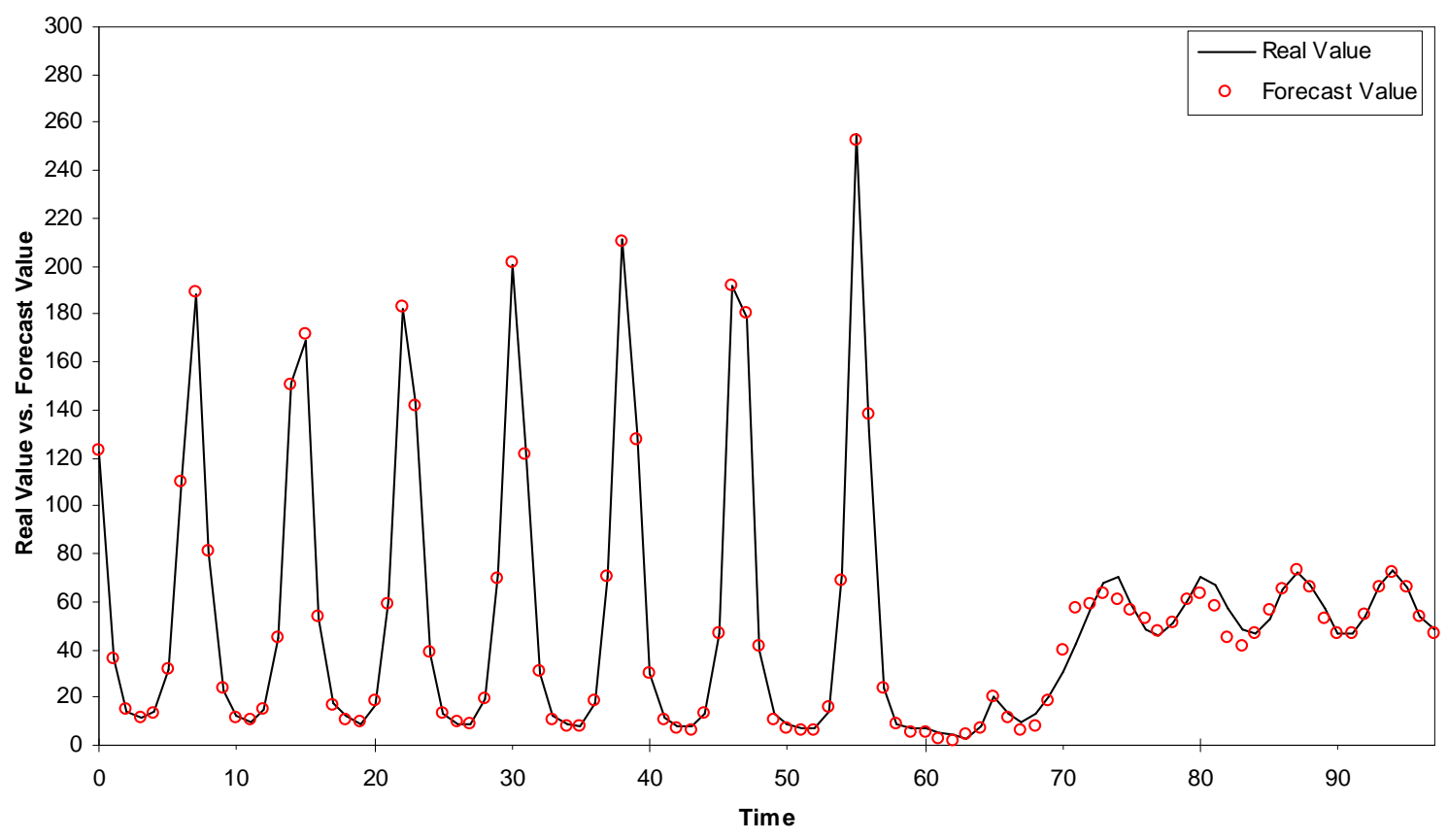

Figure 6.5 Comparison between original far-infrared NH3 laser series and final forecast values. 
Table 6.9 Comparison between the prediction errors reported in the literature and the proposed method (100 far-infrared NH3 laser time series validation data).

\begin{tabular}{|c|c|c|c|}
\hline & & Prediction & Improvement \\
\hline Prediction Method & Reference & NMSE & Percentage \\
\hline MLP & {$[28]$} & $1.72 \mathrm{E}-01$ & 98.1 \\
\hline MLP & {$[228]$} & $9.96 \mathrm{E}-02$ & 96.7 \\
\hline RSOM & {$[127]$} & 8.33E-02 & 96.1 \\
\hline SVR & [137] & $5.80 \mathrm{E}-02$ & 94.4 \\
\hline LSTM & [228] & $3.96 \mathrm{E}-02$ & 91.8 \\
\hline MS-SVR & [137] & $3.92 \mathrm{E}-02$ & 91.7 \\
\hline LSTM IT & {$[228]$} & $3.64 \mathrm{E}-02$ & 91.1 \\
\hline Elman & {$[28]$} & $3.40 \mathrm{E}-02$ & 90.5 \\
\hline NARX & {$[28]$} & $3.39 \mathrm{E}-02$ & 90.4 \\
\hline FIR-MLP & [126] & $2.30 \mathrm{E}-02$ & 85.9 \\
\hline Weigend and Nix & [229] & $1.98 \mathrm{E}-02$ & 83.6 \\
\hline MLP & [127] & $1.78 \mathrm{E}-02$ & 81.8 \\
\hline MLP IT & {$[228]$} & $1.58 \mathrm{E}-02$ & 79.5 \\
\hline BPTT & [107] & 7.92E-03 & 59.1 \\
\hline СBPTT & [107] & $5.60 \mathrm{E}-03$ & 42.1 \\
\hline EBPTT & {$[107]$} & $5.37 \mathrm{E}-03$ & 39.7 \\
\hline Boosting-Squared & [107] & $4.31 \mathrm{E}-03$ & 24.8 \\
\hline Boosting-Linear & [107] & $3.77 \mathrm{E}-03$ & 14.1 \\
\hline The Proposed Method - Taguchi's DOE & & $3.24 \mathrm{E}-03$ & \\
\hline
\end{tabular}

\subsection{Summary}

In this study, Taguchi's design of experiments was utilized to identify the factorlevel combinations which improve the performance of the proposed method to forecast chaotic time series. Based on the embedding theorem, the original time series can be 
unfolded with embedding dimension and time delay and reconstructed into the phase space points. The first neural network was trained, and the future values of the embedded phase space points and, consequently, the original time series were predicted. It was observed that, in some cases, the residuals of the forecasting method were not random and exhibited the same characteristics as the original time series. The accuracy of this prediction method was further enhanced by analyzing and incorporating the prediction residuals. The residual analysis was repeated several times. Eventually, to capture the relationship between the predicted values and the original time series, a new neural network was trained. Taguchi's design of experiments was introduced as a pre-step to the forecasting method to select proper combinations of ensemble artificial neural networks and their parameters.

The performance of the improved forecasting method has been validated for a one-step ahead prediction in terms of error indices. The results were compared with the performance of reported forecasting methods in the literature. Two systems which produce chaotic time series, Mackey-Glass and Lorenz, and two real life chaotic time series, sunspot and a far-infrared NH3 laser time series, were selected for benchmarking. In all four time series analysis, Tier Neural Network Architecture, Final Neural Network Architecture, Tier Network Training Method and Final Network Training Method showed importance and their selected levels were Elman, NARX, traingdx and trainlm, respectively. The experimental results confirm that the selected levels identified by Taguchi's DOE have improved the performance of the proposed forecasting method when compared with the same method using arbitrary selection of architectures and parameters or other existing forecasting methods in the literature. 


\section{CHAPTER 7}

\section{APPLICATIONS OF CHAOTIC TIME SERIES FORECASTING}

Applications of chaos theory are becoming increasingly widespread in a variety of disciplines. Chaos theory has been applied to the control of robot servos [4]. The implication of chaos has been observed in communications, the optimization of telephone exchanges [5] and the transmission of digital signals [6]. Data analysis methods developed for the analysis of chaotic behaviour have been applied to bulk chemical reactions [7]. Chaos theory has also been applied to economics [230,231], ecology [232], international relations [233] and medicine [234]. In the supply chain management area, the principles of chaos theory have been used in demand forecasting, product design and inventory management $[19,235,236]$.

González and Pereda [17] utilized chaos theory to study the short-term cardiovascular control system. Wichard et al. [237] applied chaos theory to the balancing problem of spinning rotors. In the hydrological field, Jayawardena and Lai [71] detected chaotic behaviour in daily rainfall and streamflow data. Feichtinger [238] has discussed the importance of chaos theory in operations research. In the financial sector, chaos theory has been applied mainly to commodity markets, stock markets, exchange rates, bankruptcy prediction and financial planning [8]. 
Prediction of nonlinear and chaotic time series is a useful method to evaluate characteristics of dynamical systems. Predictions of chaotic time series have been observed in the areas of marketing [9,239], foreign exchange rates [10,102], signal processing [11], supply chain management [12,19], oil price and consumption [240], traffic flow [13,241], power load [14,242], weather forecasting [15], tidal prediction [72], sunspot prediction [16], air quality [243], production systems [237] and many others. Due to the importance of these fields, the interest in a robust technique to predict chaotic time series has increased.

In Chapter 6, the final form of the proposed forecasting method was presented. This original forecasting method was developed using the embedding theorem, ensemble neural networks, residual analysis, genetic algorithms and Taguchi's design of experiments. In the current chapter, the proposed method is applied to financial markets, namely, stock markets and exchange rate trading. In the following sections, the daily price of four stocks, namely, British Airlines, Southwest Airlines, Ryanair Holdings and Lucent Incorporated, and two exchange rates, namely, EUR/USD and USD/JPY, are analyzed and their future values are predicted.

\subsection{Stock Markets}

In the last few decades, stock markets have become an inseparable part of the global economy. Any change in stock markets impacts on the financial status of individuals, corporations and countries [244,245]. The crash of stock markets on October 19, 1987, increased the interests of macroeconomists and financial researchers to investigate chaotic behaviour in stock markets. They observed that the large movements 
in stock markets are greater than the expected normal distribution. Later, financial economists concluded that stock markets are governed by chaotic dynamics [246].

The relationship between chaos theory and stock markets has been discussed repeatedly in the literature $[239,246,247,248]$. In the financial sector, chaos theory has been applied mainly to commodity markets, stock markets, exchange rates, bankruptcy prediction, financial planning [8] and employment rates [239]. The research work by Baumol and Benhabib [230] presents a comprehensive survey of economic models which deal with chaotic behaviour of stock markets [246].

Forecasting the future of stock markets is a challenging and attractive subject [9]. Stock markets are evolutionary, nonlinear and complex systems [249]. Numerous factors are involved in prediction of stock markets including traders' expectations, general economic conditions and political events $[139,250]$. There is always some degree of risk in investing in stock markets due to the difficulties in prediction of the stock market movements [245]. Therefore, an appropriate forecasting model could decrease the risk of such investments.

Saad et al. [251] utilized time delay neural networks (TDNN), recurrent neural networks (RNN) and probabilistic neural networks (PNN) to forecast Apple stock trends. Huanga et al. [250] investigated the predictability of financial movement direction with a support vector machine (SVM) and applied the method to the weekly movement direction of NIKKEI 225 stock data. Hassan and Nath [245] utilized a hidden Markov model (HMM) approach to forecast stock prices for some airline stocks: Southwest Airlines, British Airlines, Delta Airlines and Ryanair Holdings Ltd. Qian and Rasheed [9] used the Hurst exponent to determine a period with great predictability for 
the Dow Jones Industrial Average index. Afolabi and Olude [139] used backpropagation, a Kohonen self-organizing map (SOM), and a hybrid Kohonen SOM to forecast the daily price of Lucent Incorporated stock.

In this section, the proposed forecasting method using chaos theory, the embedding theorem, residual analysis and improved ensemble neural networks with genetic algorithms and Taguchi's design of experiments is applied to the daily price of four stocks: British Airlines, Southwest Airlines, Ryanair Holdings Ltd. and Lucent Incorporated.

\subsubsection{British Airlines Stock Data}

The daily price of British Airlines stock has been selected for analysis and forecasting. In order to compare the performance of the proposed forecasting method in this study when applied to British Airlines stock data with the results reported in the literature, the conditions are set based on Hassan and Nath [245]. The daily stock data of British Airlines for the period January 1, 2003 to September 10, 2004 which includes 443 data is selected for training of the developed model in this study. Accordingly, the daily stock data for the period September 11, 2004 to January 10, 2005 which includes 94 data is selected for testing of the proposed method to forecast one step ahead for the closing price of British Airlines stock. Figure 7.1 shows the daily closing price of British Airlines stock for the period January 1, 2003 to January 10, 2005.

The first step in applying the proposed forecasting method to British Airlines stock data is to detect chaos in the time series. The Lyapunov exponent is the most popular method to investigate the existence of chaos in a given time series [10,252,253,254]. A 
positive Lyapunov exponent confirms the existence of chaos. Figure 7.2 shows the prediction error versus the length of prediction for British Airlines stock data.

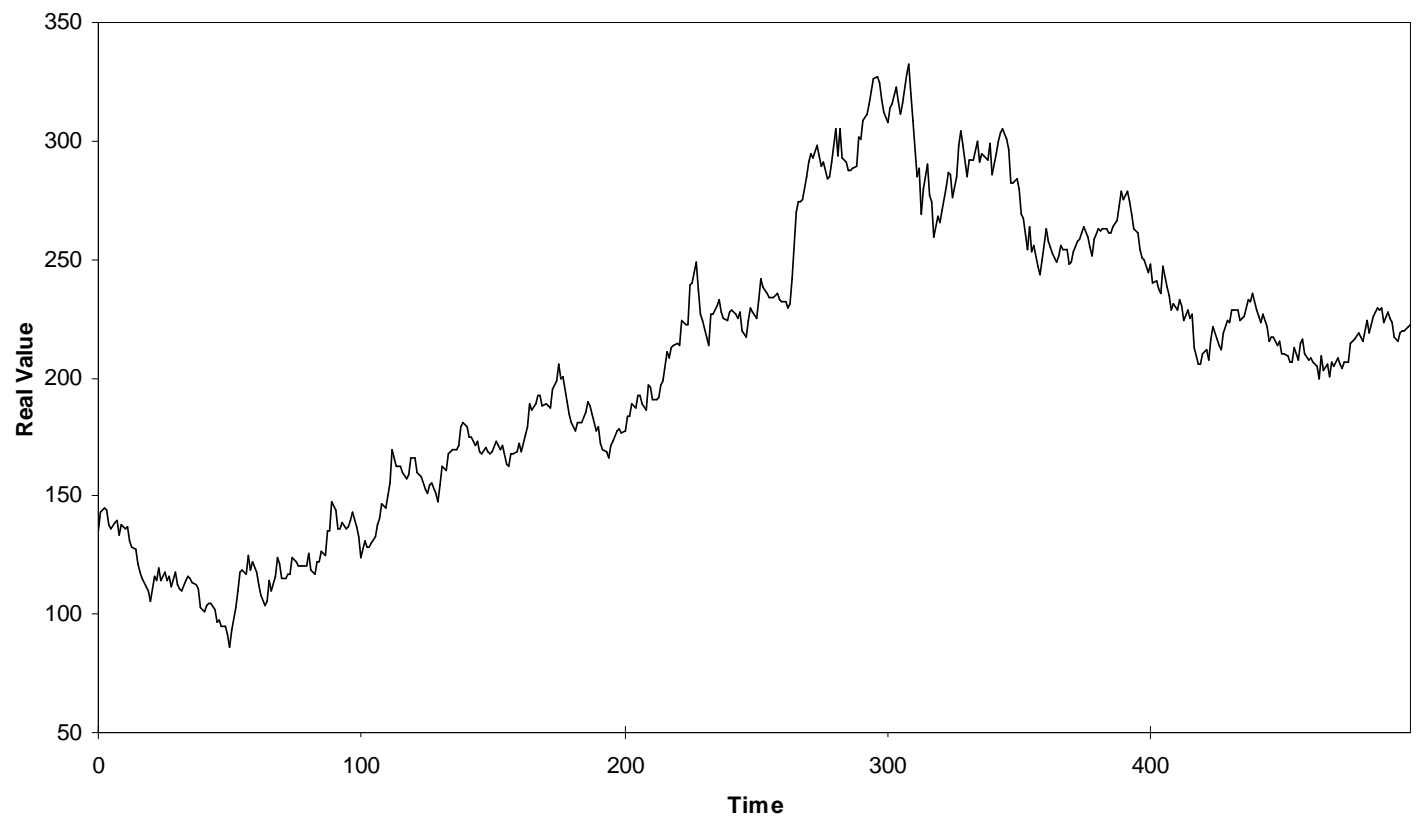

Figure 7.1 The daily closing price of British Airlines stock for Jan. 1, 2003 to Jan. 10, 2005.

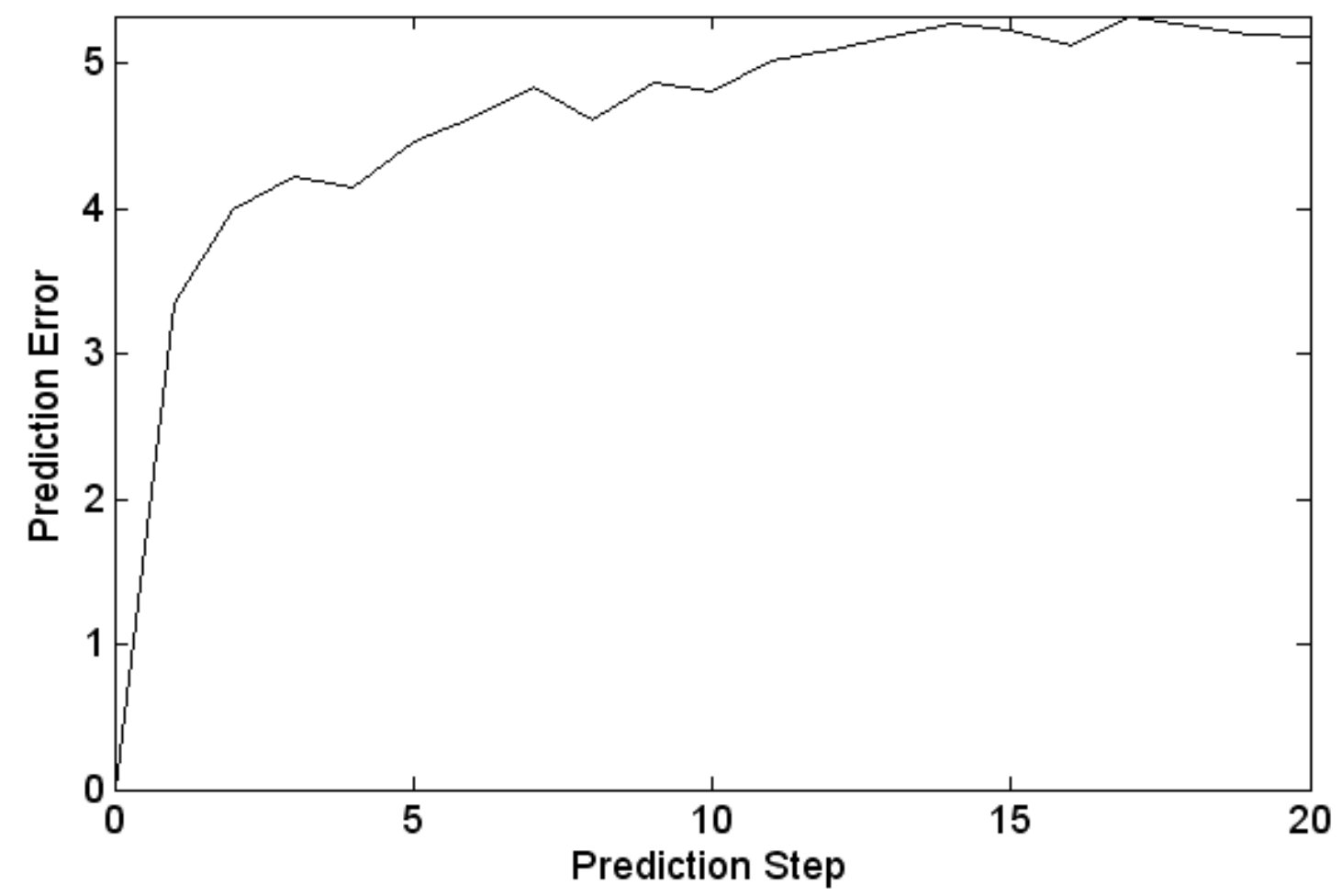

Figure 7.2 Largest Lyapunov exponent graph for British Airlines stock data. 
The slope of the linear portion of the graph estimates the value of the largest Lyapunov exponent (LLE). The largest Lyapunov exponent for British Airlines stock data is positive, $\operatorname{LLE}=3.36$, which is an indication of chaotic behaviour in the data.

The embedding dimension $D$ and the time delay $T$ are calculated using TSTOOL [190]. British Airlines stock data is reconstructed into phase space points with embedding dimension $D=3$ and time delay $T=1$. The data in the phase space domain are shown in Figure 7.3.

The embedded phase space points are fed into an Elman neural network with 3 neurons in the input and output layers and 6 hidden and recurrent neurons. The weights and biases of the trained Elman neural network are kept to forecast the future values of the phase space points and eventually the future values of British Airlines stock data. Figure 7.4 compares the real data and the predicated values of 94 data kept for testing.

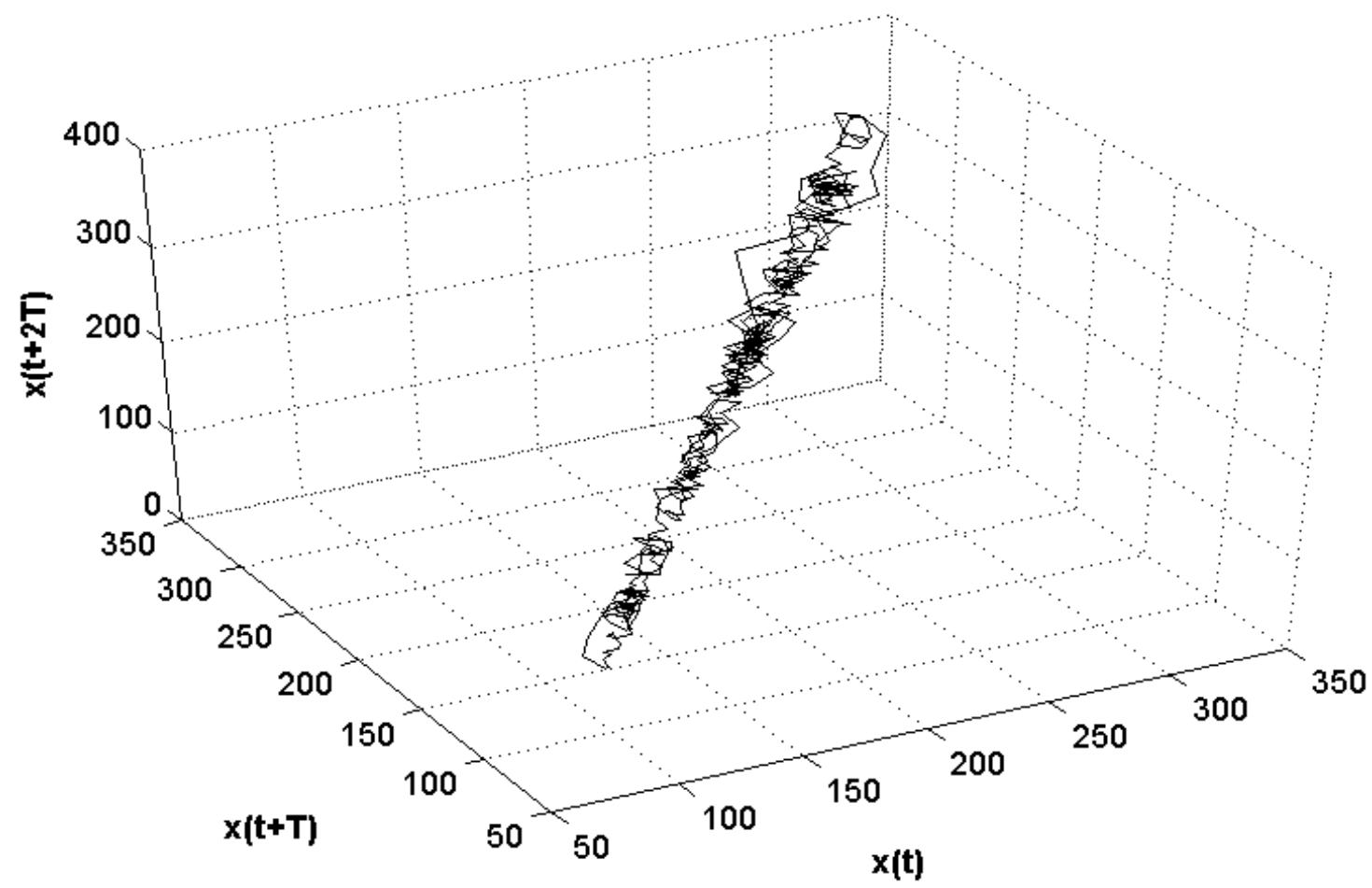

Figure 7.3 British Airlines stock data in phase space domain. 


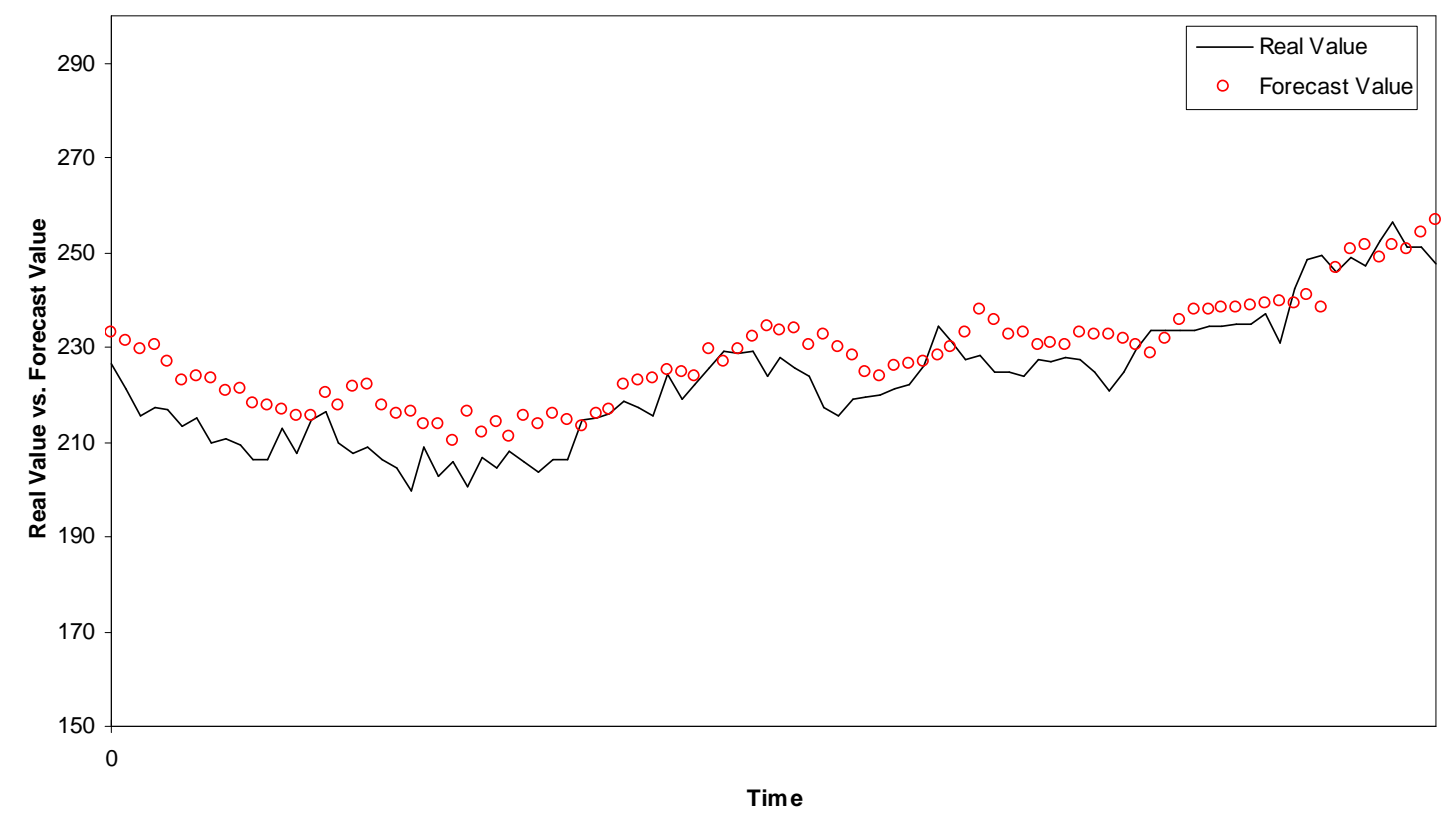

Figure 7.4 British Airlines stock data - Comparing the original stock data and the predicted values.

The prediction errors for the training data are calculated and analyzed. The largest Lyapunov exponent LLE $=7.24$ is determined for the residual time series using TSTOOL [190], which shows the existence of chaos in the obtained residuals. Based on this finding, the residuals are treated as a new chaotic time series and predicted. The first $(D-1) T+1$ data have been used for the first prediction; therefore, the length of the residual time series is $(D-1) T+1$ shorter than the original time series. The residuals may not have the same embedding parameters as the original time series; therefore, they need to be analyzed independently. The 440 residuals obtained from the original prediction of the training data are reconstructed using the estimated embedding dimension $D_{1}=4$ and time delay $T_{1}=1$. The embedded phase space points are fed into a new Elman neural network. The neural network is set up with 4 neurons in the input and output layers and 6 hidden and recurrent neurons. Figure 7.5 presents the first residuals and their predicted values for the test data. 


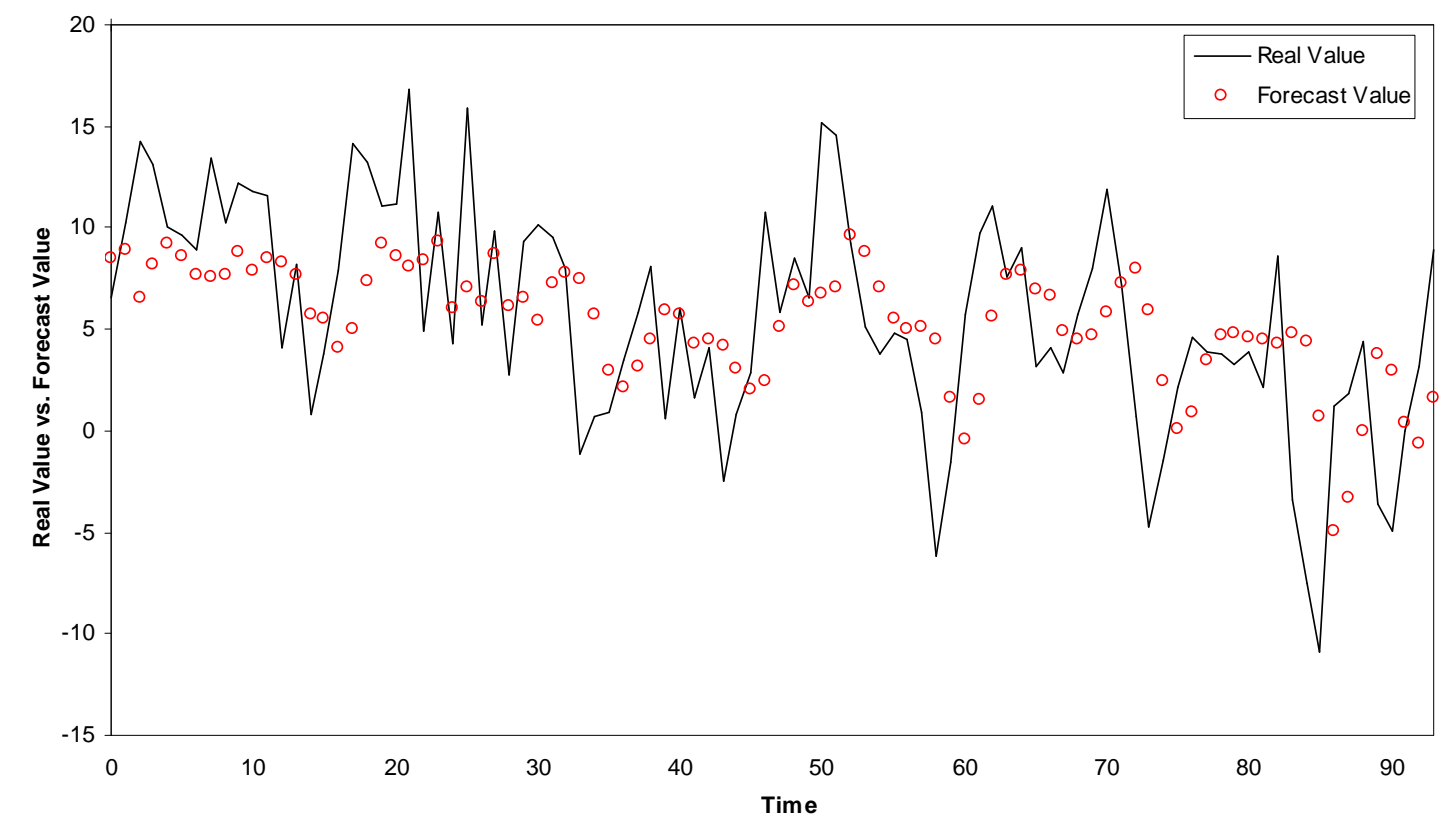

Figure 7.5 British Airlines stock data - The comparison of the first residual values and the predicted values.

The residual analysis is repeated one more time. The largest Lyapunov exponent LLE $=7.58$ is determined for the residual time series using TSTOOL [190], which shows the existence of chaos in the obtained residuals. After confirmation that the residuals are chaotic, with $D_{2}=4$ and time delay $T_{2}=1$, the second order residuals are reconstructed and predicted using an Elman neural network. Figure 7.6 presents the comparison between the second order residual values and their predicted values.

Finally, a NARX neural network is trained using the neural networks' result values of the time series and the residuals as input and the original time series as output. The NARX neural network is used to capture the relationship between the predicted values and the original time series. The initial weights and biases of the final neural networks are improved using genetic algorithms. Figure 7.7 compares the original values of British Airlines stock data with their predicted values. 


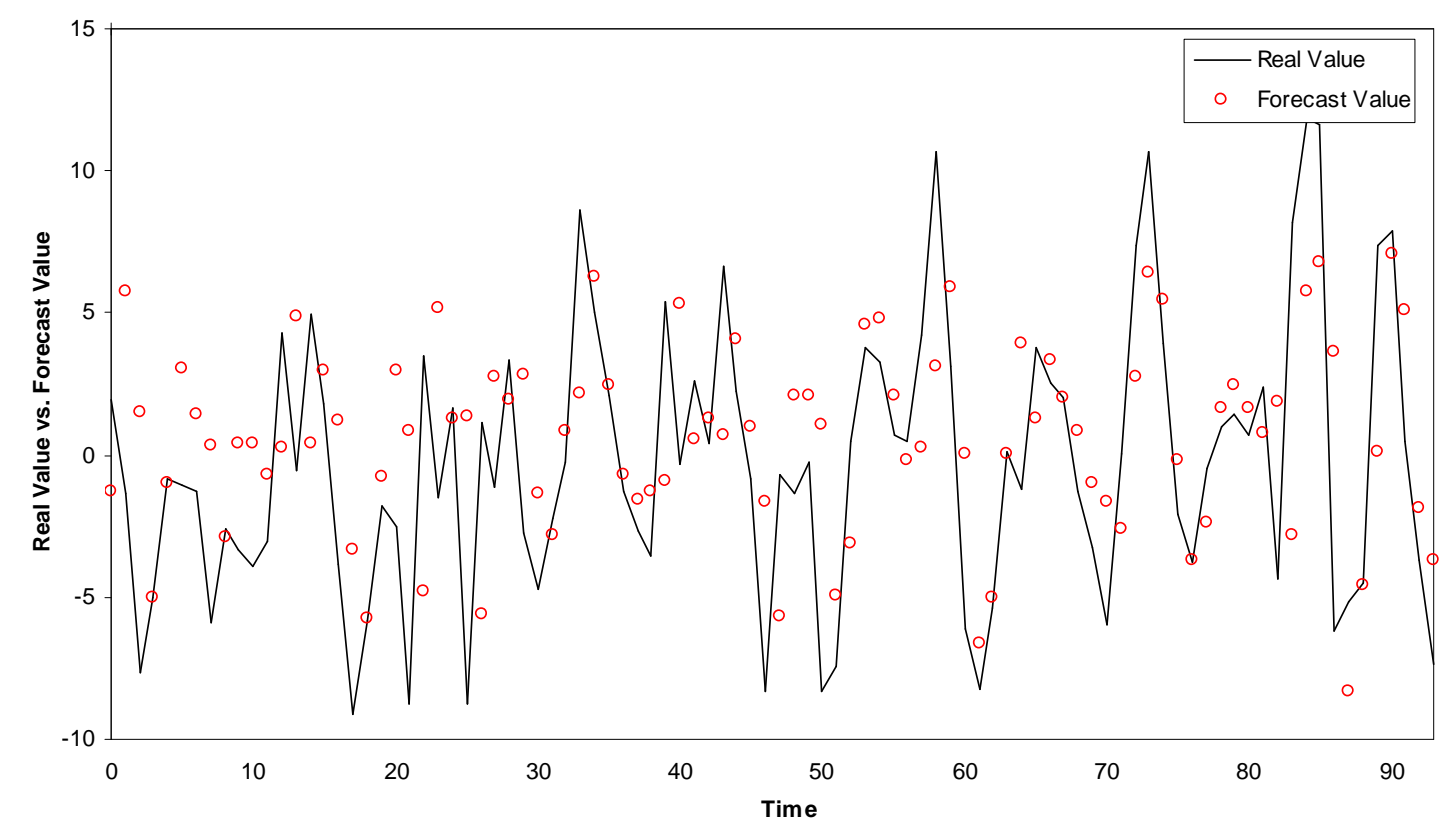

Figure 7.6 British Airlines stock data - The comparison of the second residual values and the predicted values.

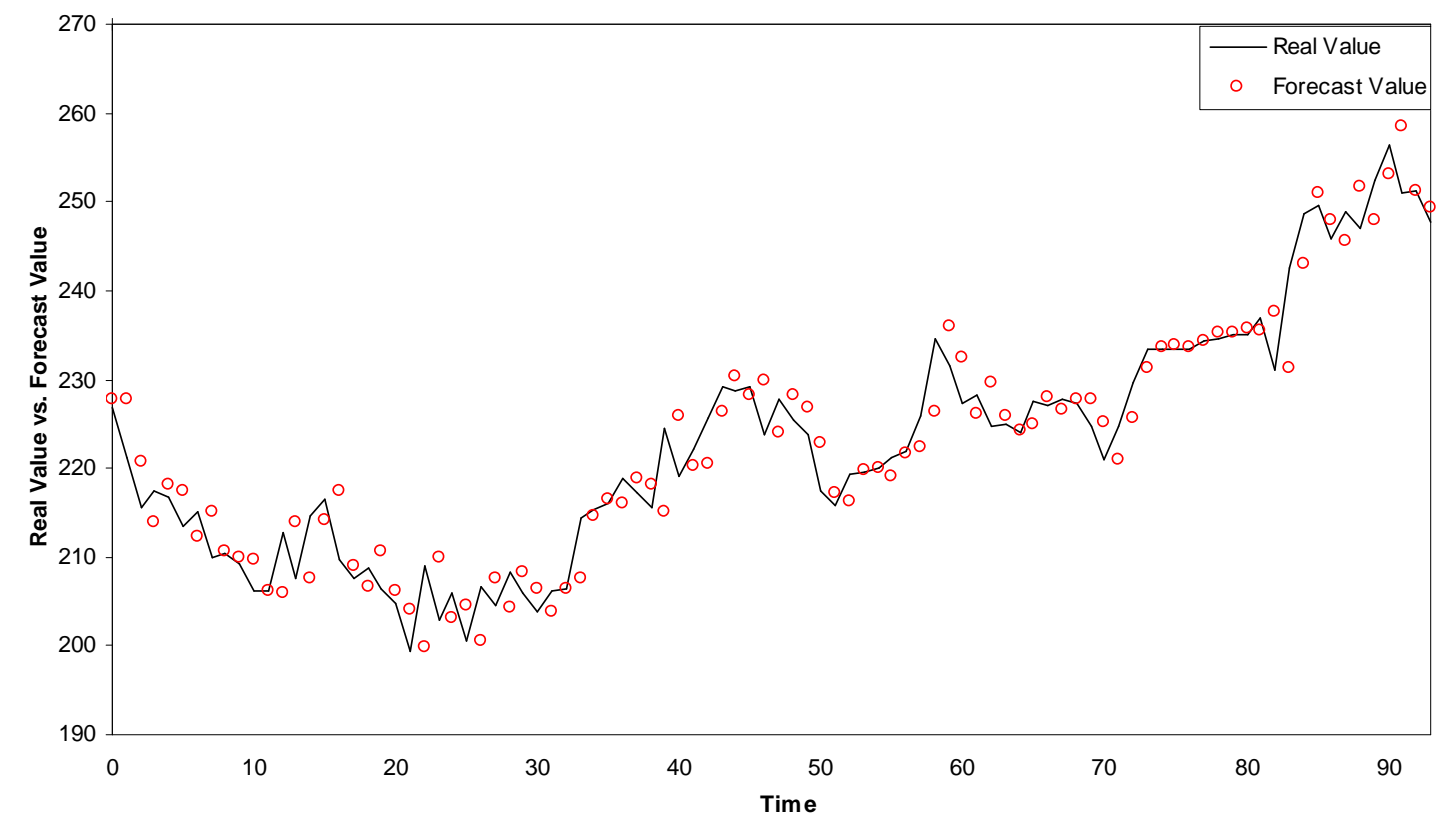

Figure 7.7 The comparison between original British Airlines stock data and the final predicted values. 
In order to evaluate the prediction performance and compare it with the results reported by Hassan and Nath [245], the mean absolute percentage error (MAPE) index is calculated according to Eq. (37):

$M A P E=\frac{1}{N} \sum_{i=1}^{N}\left|\frac{y_{i}-\hat{y}_{i}}{y_{i}}\right|$

where $y_{i}$ and $\hat{y}_{i}$ are observed data and predicted data, respectively. The term $N$ is the length of observed data.

The prediction accuracy for 94 British Airlines stock test data, in the demonstrated experiment based on the improved Elman-NARX configuration, is compared with the results reported in the literature. Table 7.1 presents the comparison between the prediction errors reported in the literature and that of the proposed method for British Airlines stock data. This experiment confirms that the proposed forecasting method, when it is used to forecast the future values of British Airlines stock data, generates better results compared to other prediction methods reported in the literature.

Table 7.1 Comparison between the prediction errors reported in the literature and the proposed method (94 British Airlines stock test data).

Prediction Error Improvement

\begin{tabular}{llll}
\hline Prediction Method & Reference & MAPE & Percentage \\
\hline Hidden Markov Model (HMM) & {$[245]$} & 2.629 & 99.4 \\
Artificial Neural Network (ANN) & {$[245]$} & 2.283 & 86.3 \\
The Proposed Method - Taguchi's DOE & -- & $1.45 \mathrm{E}-02$ & \\
\hline
\end{tabular}




\subsubsection{Southwest Airlines Stock Data}

For the second real life numerical experiment, the daily price of Southwest Airlines stock has been selected. In order to compare the performance of the proposed forecasting method in this study when applied to Southwest Airlines stock data with the results reported in the literature, the conditions are set based on Hassan and Nath [245]. The daily stock data of Southwest Airlines for the period December 18, 2002 to July 23, 2004 which includes 401 data is selected for training of the developed model in this study. Accordingly, the daily stock data for the period July 24, 2004 to November 17, 2004 which includes 82 data is selected for testing of the proposed method to forecast one step ahead for the closing price of Southwest Airlines stock. Figure 7.8 shows the daily closing price of Southwest Airlines stock for the period December 18, 2002 to November 17, 2004.

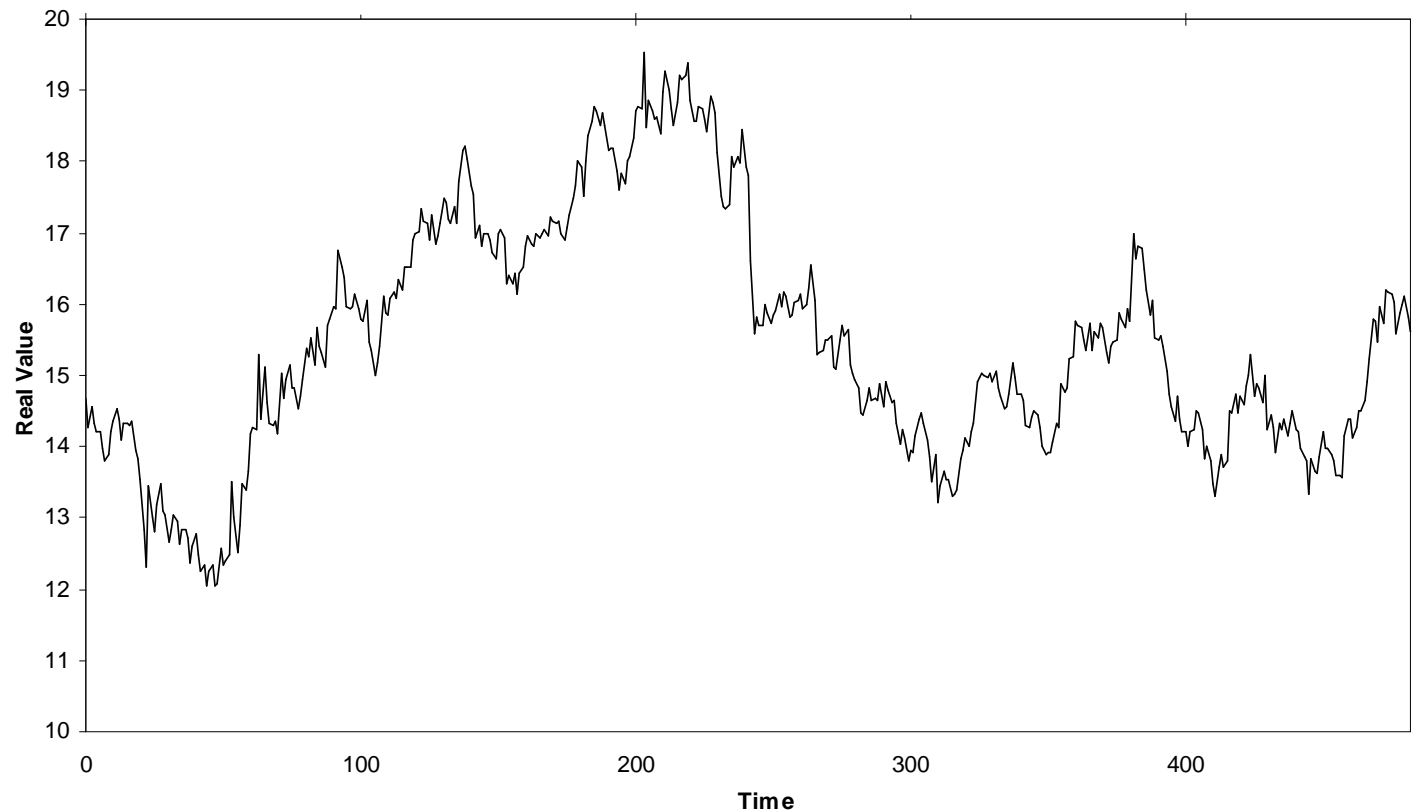

Figure 7.8 The daily closing price of Southwest Airlines stock December 18, 2002 to November 17, 2004. 
The first step in applying the proposed forecasting method to Southwest Airlines stock data is to detect chaos in the time series. The largest Lyapunov exponent for Southwest Airlines stock data is positive, LLE $=4.08$, which indicates chaotic behaviour.

The original time series is reconstructed with embedding dimension $D=4$ and time delay $T=1$. The embedded phase space points are fed into an Elman neural network with 4 neurons in the input and output layers and 6 recurrent neurons and trained. The weights and biases of the Elman neural network are kept to forecast the future values of phase space points and consequently the original time series.

Two levels of residual analysis are performed to increase the accuracy of the prediction. Based on a positive largest Lyapunov exponent, LLE $=7.26$, it is concluded that the first order residuals are chaotic. The first order residuals, are reconstructed with embedding dimension $D_{1}=3$ and time delay $T_{1}=1$. The reconstructed phase space points are fed into a new Elman neural network. The neural network is set up with 3 neurons in the input and output layers and 6 neurons in the hidden layer. The residual analysis is repeated one more time and it is concluded that the second order residuals show chaotic behaviour, LLE $=7.67$. With $D_{2}=3$ and time delay $T_{2}=1$, the future values of the second order residuals are predicted based on the proposed prediction method. Finally, a NARX network is trained using neural networks' result values of the time series and the residuals as input and the original time series as output. The last neural network is used to capture the relationship between the neural networks' result values and the original time series. Figure 7.9 compares the original values of Southwest Airlines time series with their final predicted values. 


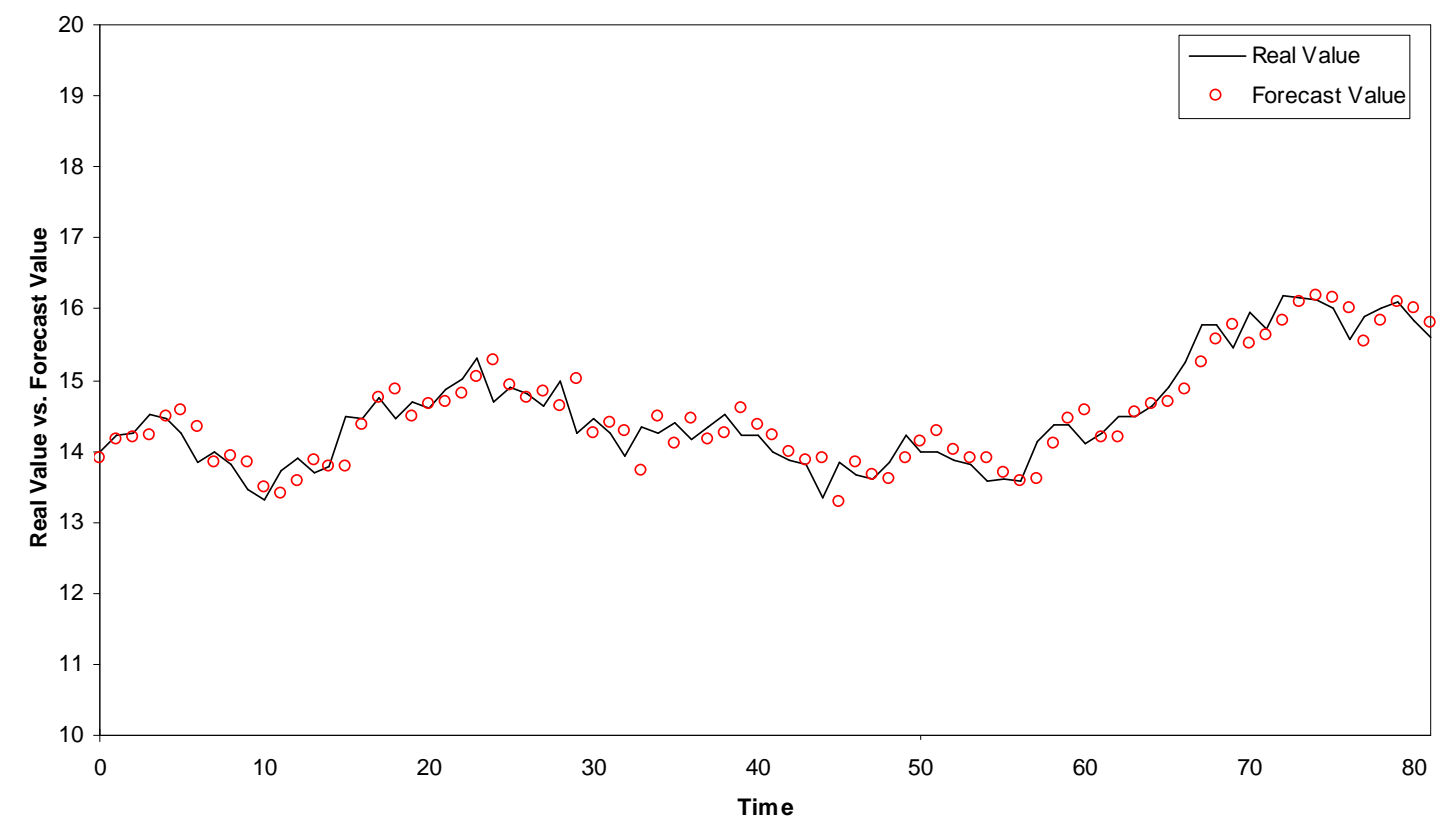

Figure 7.9 The comparison between original Southwest Airlines stock data and the final predicted values.

The error index, MAPE for the 82 Southwest Airlines test samples is compared with the results reported in the literature and presented in Table 7.2. The experiment performed confirms that the performance of the proposed method with improved ElmanNARX configuration is better in prediction of Southwest Airlines time series when compared to other prediction methods reported in the literature.

Table 7.2 Comparison between the prediction errors reported in the literature and the proposed method (82 Southwest Airlines stock test data).

Prediction Error Improvement

\begin{tabular}{llll}
\hline Prediction Method & Reference & MAPE & Percentage \\
\hline Hidden Markov Model (HMM) & {$[245]$} & 2.011 & 99.2 \\
Artificial Neural Network (ANN) & {$[245]$} & 1.673 & 82.4 \\
The Proposed Method - Taguchi's DOE & -- & $1.65 \mathrm{E}-02$ & \\
\hline
\end{tabular}




\subsubsection{Ryanair Holdings Ltd. Stock Data}

The next numerical experiment is performed with the daily price of Ryanair Holdings Ltd. stock data. In order to compare the performance of the proposed forecasting method in this study when applied to Ryanair stock data with the results reported in the literature, the conditions are set based on Hassan and Nath [245]. The daily stock data of Ryanair for the period May 06, 2003 to December 06, 2004 which includes 401 data is selected for training of the developed model in this study. Accordingly, the daily stock data for the period December 07, 2004 to March 17, 2005 which includes 70 data is selected for testing of the proposed method to forecast one step ahead for the closing price of Ryanair stock. Figure 7.10 shows the daily closing price of Ryanair stock for the period May 06, 2003 to March 17, 2005.

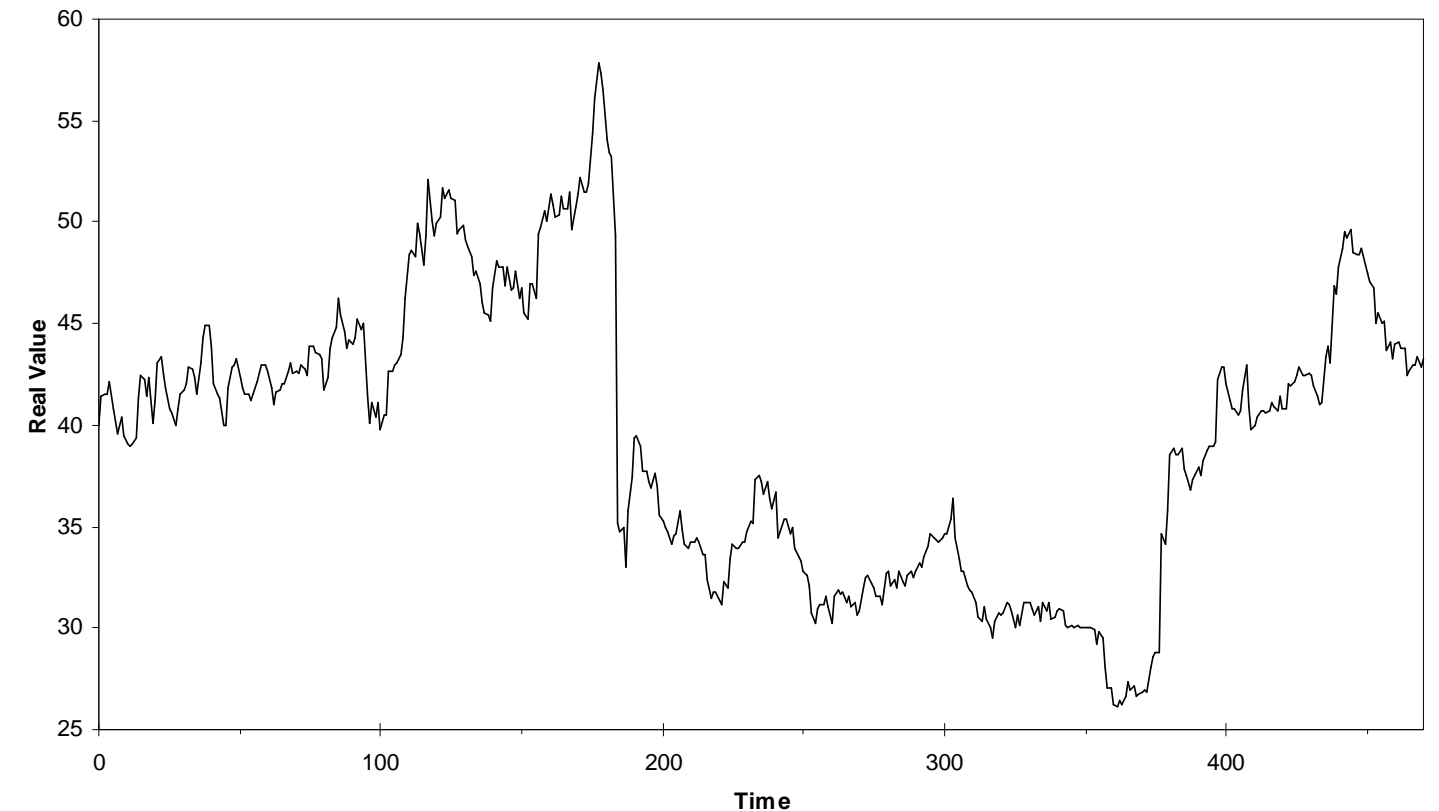

Figure 7.10 The daily closing price of Ryanair stock for the period May 06, 2003 to March 17, 2005. 
The existence of chaos is investigated in Ryanair stock data. The value of the largest Lyapunov exponent for Ryanair stock data is positive, LLE $=4.14$, which is an indication of chaotic behaviour in the data.

The embedding dimensions $D=3$ and $T=1$ are estimated as explained in Section 3.2.4 and 3.2.5 using TSTOOL [190]. Accordingly, phase space points are reconstructed. An Elman neural network including 3 neurons in the input layer, 6 neurons in the hidden layer, 6 recurrent neurons and 3 neurons in the output layer is trained. The weights and biases of the network are kept for the purpose of prediction.

The prediction errors for the data selected for the training phase are calculated and analyzed. The largest Lyapunov exponent, LLE $=7.04$ is positive; therefore, it is concluded that the residuals show chaotic behaviour. Based on this finding, the residuals are treated as a new chaotic time series with embedding dimension $D_{1}=5$ and time delay $T_{1}=1$ and the proposed method is applied to predict the future value of the residuals. The second level of residual analysis is performed and the existence of chaos is detected in the residual time series, $\mathrm{LLE}=6.86$. With the embedding dimension $D_{2}=3$ and time delay $T_{2}=1$, the second level residuals are predicted. Finally, a NARX network is trained using neural networks' result values of the time series and the residuals as input and the original time series as output to correlate the predicted values to the original time series. Figure 7.11 compares the original values of Ryanair time series with their final predicted values.

The error index, MAPE, for the 70 Ryanair stock test samples are compared with the results reported in the literature and presented in Table 7.3. The experiment performed for Ryanair stock data confirms that the proposed method with the improved 
Elman-NARX configuration performs better than other prediction methods reported in the literature.

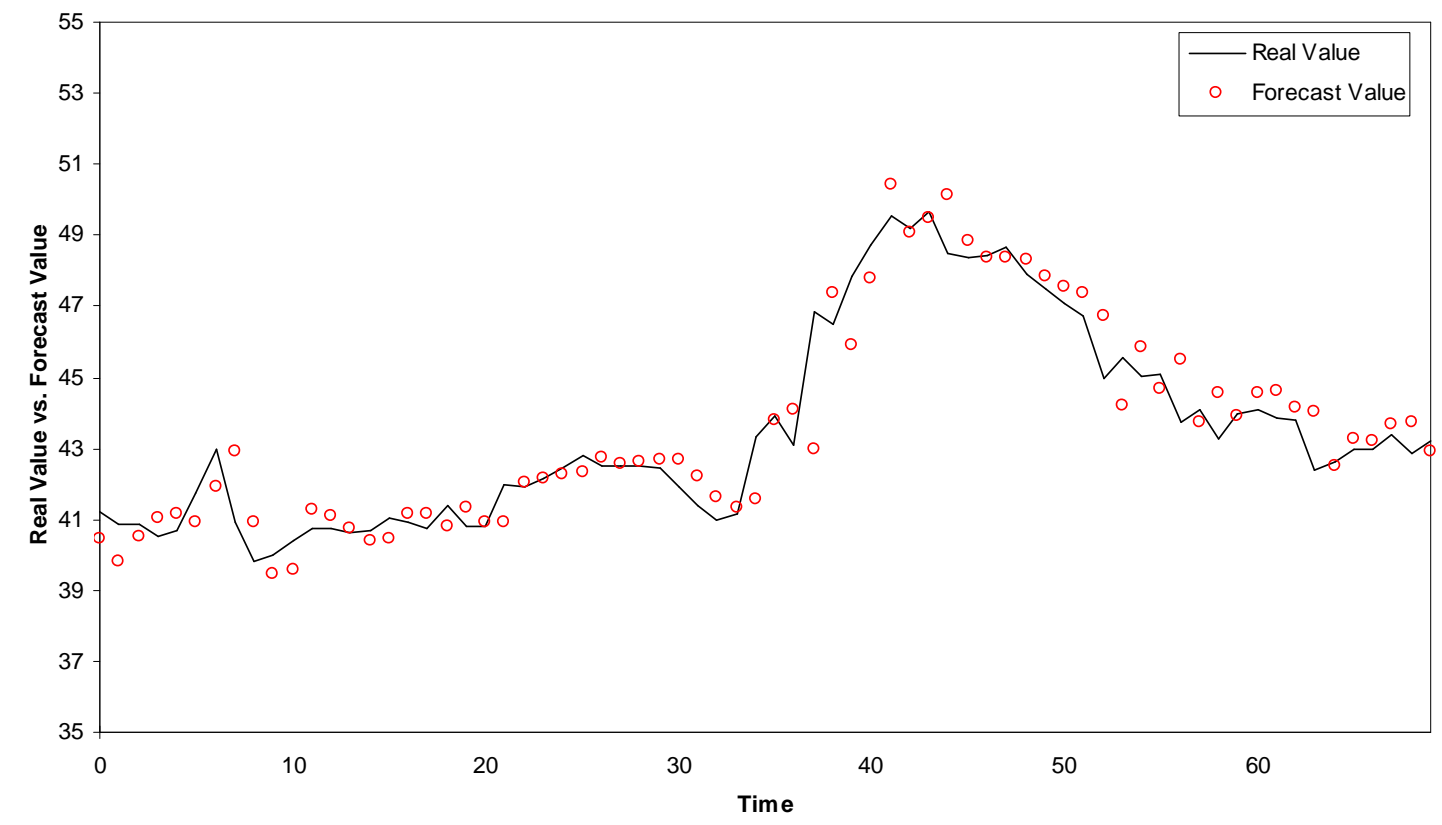

Figure 7.11 The comparison between original Ryanair stock data and the final predicted values.

Table 7.3 Comparison between the prediction errors reported in the literature and the proposed method (70 Ryanair stock test data).

Prediction Error Improvement

\begin{tabular}{llll}
\hline Prediction Method & Reference & MAPE & Percentage \\
\hline Hidden Markov Model (HMM) & {$[245]$} & 1.492 & 99.0 \\
Artificial Neural Network (ANN) & {$[245]$} & 1.928 & 128.2 \\
The Proposed Method - Taguchi's DOE & -- & $1.56 \mathrm{E}-02$ & \\
\hline
\end{tabular}




\subsubsection{Lucent Incorporated Stock Data}

The last numerical experiment performed on stock data in this study is the daily stock price of Lucent incorporated. In order to compare the performance of the proposed forecasting method in this study when applied to Lucent incorporated stock data with the results reported in the literature, the conditions are set based on Afolabi and Olude [139]. The daily stock data of Lucent incorporated for the period November 28, 1998 to November 28, 2003 which includes 1258 data is selected for training and testing of the developed model in this study. Similar to Afolabi and Olude [139] the first 258 are removed and the next 500 data are selected for training and the last 500 data are used to test the performance of the proposed forecasting method. Figure 7.12 shows the scaled daily closing price of Lucent stock for the period November 28, 1998 to November 28, 2003 scaled according to Afolabi and Olude [139].

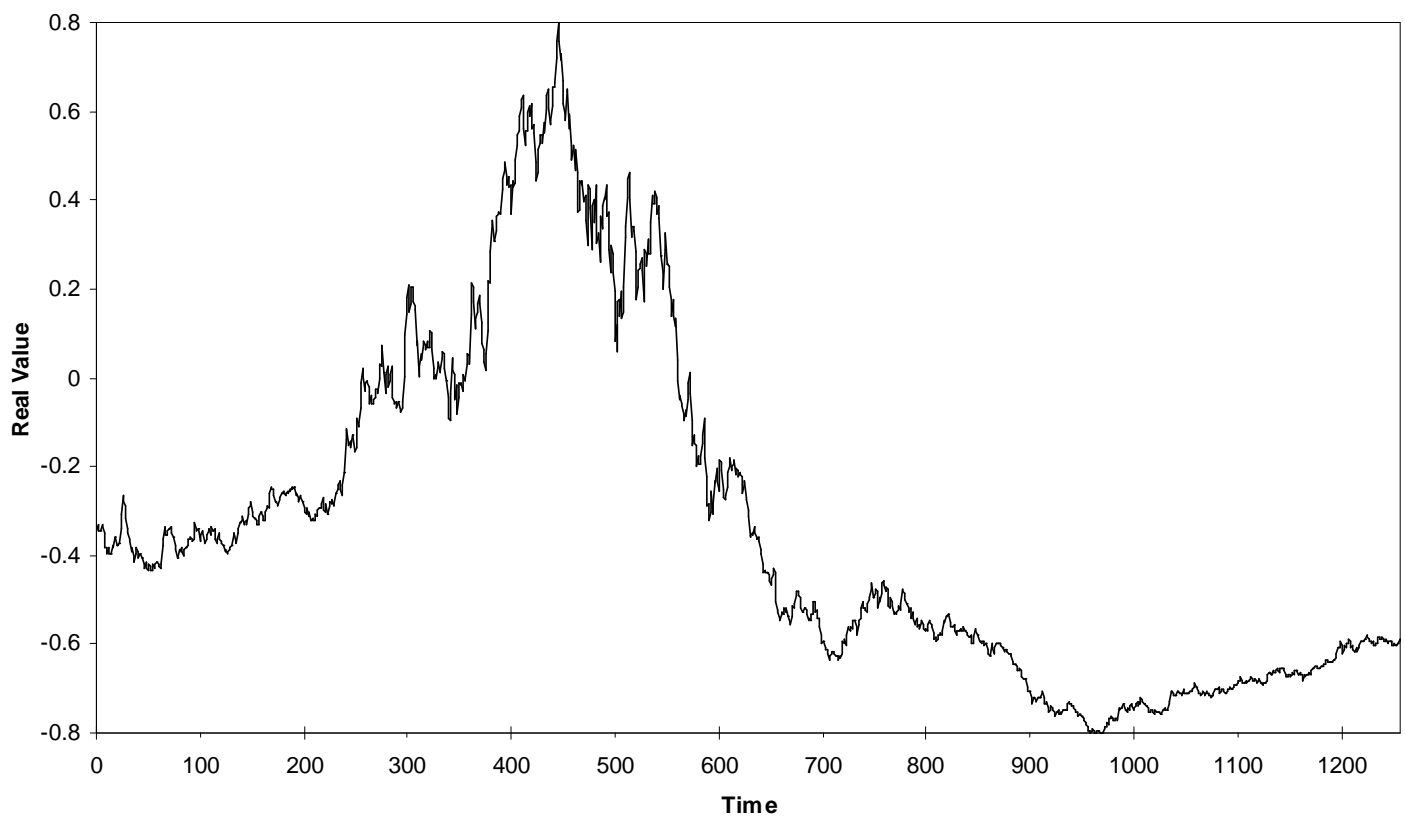

Figure 7.12 The scaled daily closing price of Lucent incorporated stock for the period November 28, 1998 to November, 282003. 
Lucent stock data are analyzed to detect chaos in the time series. The largest Lyapunov exponent for Lucent stock data is positive, LLE $=4.29$, which is an indication of chaotic behaviour in the data. The original time series is reconstructed with the embedding dimension $D=3$ and time delay $T=8$. The embedded phase space points are fed into an Elman neural network with 3 neurons in the input and output layers and 6 recurrent neurons and trained. The weights and biases of the Elman neural network are kept to forecast the future values of phase space points and consequently the original time series.

Two levels of residual analysis are performed to increase the accuracy of the prediction. Based on a positive largest Lyapunov exponent, $\mathrm{LLE}=6.56$, it is concluded that the first level residuals are chaotic. The first order residuals are reconstructed with the embedding dimension $D_{1}=3$ and time delay $T_{1}=1$. The reconstructed phase space points are fed into a new Elman neural network. The neural network is set up with 3 neurons in the input and output layers and 6 neurons in the hidden layer. The residual analysis is repeated one more time. The second level residuals are examined and with LLE $=6.56$, the existence of chaos is confirmed. With $D_{2}=3$ and time delay $T_{2}=1$, the future values of the second order residuals are predicted based on the proposed prediction method. Finally, a NARX network is trained using neural networks' result values of the time series and the residuals as input and the original time series as output. Figure 7.13 compares the scaled values of Lucent time series with their final predicted values. 


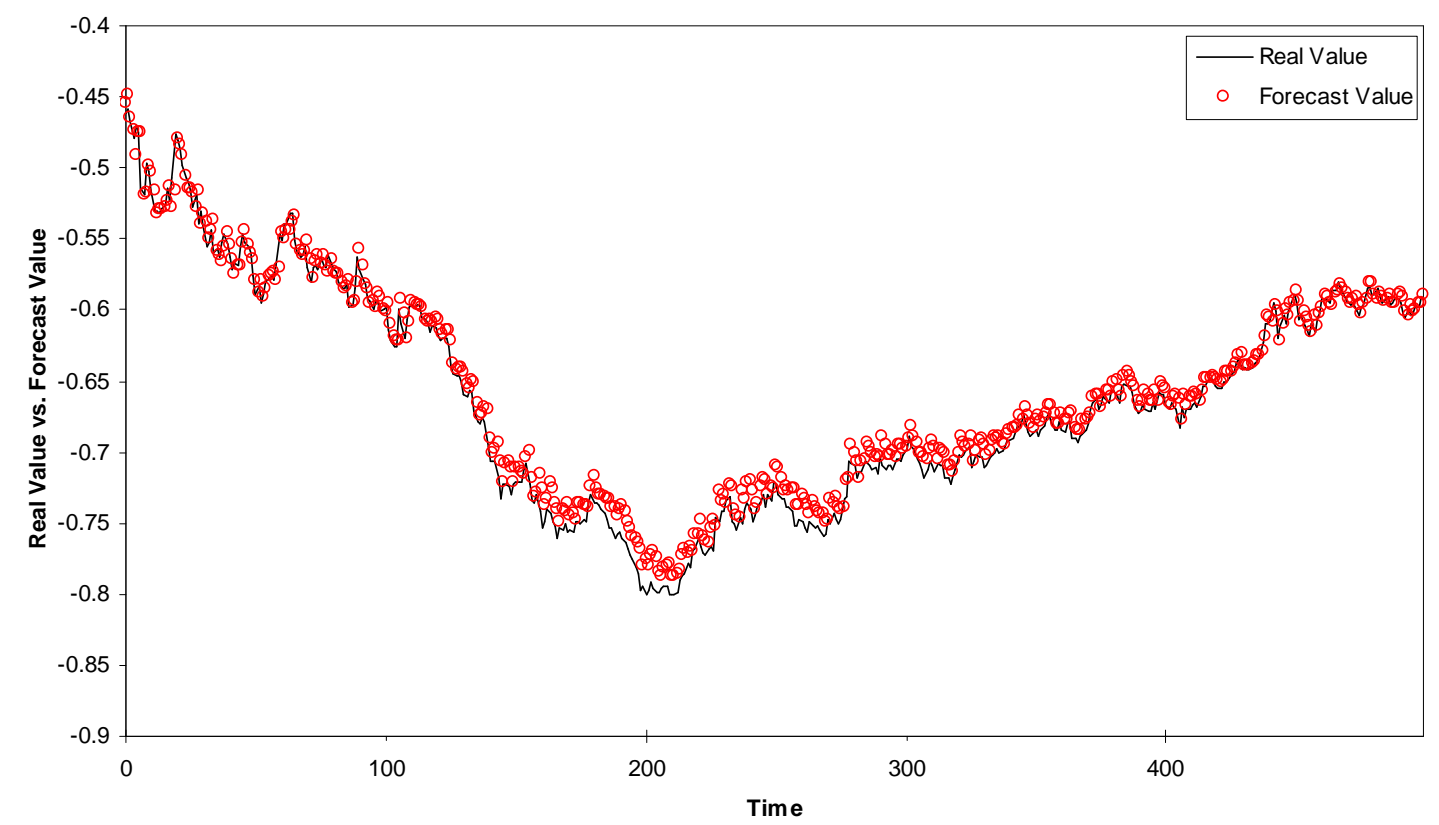

Figure 7.13 The comparison between scaled Lucent stock data and the final predicted values.

Table 7.4 presents the comparison between the prediction errors, RMSE, reported in the literature and the proposed method for 500 Lucent test samples. The results show that the proposed forecasting method with the improved Elman-NARX configuration performs better in prediction of Lucent stock data when compared to other prediction methods reported in the literature.

Table 7.4 Comparison between the prediction errors reported in the literature and the proposed method (500 Lucent stock test data).

\begin{tabular}{llll}
\hline & \multicolumn{3}{c}{ Prediction Error Improvement } \\
\hline Prediction Method & Reference & RMSE & Percentage \\
\hline Kohonen SOM Network & {$[139]$} & $1.33 \mathrm{E}-01$ & 91.4 \\
Hybrid Kohonen SOM Network & {$[139]$} & $7.23 \mathrm{E}-02$ & 45.8 \\
Backpropagation Neural Network (BPN) & {$[139]$} & $1.74 \mathrm{E}-02$ & 4.5 \\
The Proposed Method - Taguchi's DOE & -- & $1.14 \mathrm{E}-02$ & \\
\hline
\end{tabular}




\subsection{Exchange Rates}

Foreign exchange markets are one of the largest financial markets [255]. The foreign exchange trades are happening 24 hours a day in financial markets [10]. Due to the importance of foreign currencies, the exchange rates are important indicators in the international financial markets [255]. A number of fundamental factors which change the foreign exchange rates are the inflation in the concerned country, the supply of money, the money market rate as a measurement for the short-term interest rate, the long-term government bond yield and lending rate, which are the measurement for the long-term interest rate, the rate of industrial production and the trade balance relative to the gross domestic product (GDP) [10].

The exchange rate forecast models are generally categorized into two types: fundamental and technical. The fundamental model is based on the feedback of the demand and supply of domestic currency compared with a foreign currency. In the technical model, the historical exchange rate data are required to determine the underlying structure of these data and obtain the governing rules by which the time series data are produced $[255,256]$. The developed forecasting method in this study falls under technical models.

The evidence of complexity, nonlinearity and dynamical behaviour has been observed in the exchange rate time series $[257,258,259,260,261]$. Cecen et al. [252] and Bask $[253,254]$ showed the existence of chaos in the exchange rate markets by obtaining a positive Lyapunov exponent in the exchange rate time series. Richards [262] and Schwartz and Yousefi [263] detected fractal properties in the exchange rate time series and claimed chaotic behaviour in the exchange rate markets. Das and Das [10] investigated nonlinearity and chaos in foreign exchange rates for twelve countries: 
Australia, Canada, China, India, Japan, Malaysia, Singapore, Sri Lanka, Sweden, Switzerland, Thailand and UK, over the span of nearly 36 years. Calculating the largest Lyapunov exponent (LLE), Das and Das [10] found an indication of chaotic behaviour in all the exchange rate time series.

A number of methods are introduced in the literature to forecast foreign exchange rates. Tenti [102] proposed the application of recurrent neural networks to predict foreign exchange rates. The method developed by Tenti was used to forecast the future of Deutsche mark currency. Kim and Kim [154] used a genetic fuzzy predictor ensemble (GFPE) method to forecast the exchange rates between the Swiss franc and the US dollar (CHF/USD). Guegan and Mercier [264] developed two forecasting methods based on the nearest neighbours and the radial basis function to forecast the daily exchange rates between the Deutsche mark and the French franc (DEM/FRF). Liu [255] selected fuzzy logic to study exchange rates between the US dollar and the Japanese yen (USD/JPY) and developed a dynamic adaptive neuron-fuzzy logic forecasting model. Sheikhan and Movaghar [73] proposed an evolutionary connectionist model in which GA is used to improve the parameters and structure of the feedforward neural network. The proposed method by Sheikhan and Movaghar [73] was applied to the daily exchange rates between the Euro and the US dollar (EUR/USD) and the Euro and the Britain pound (EUR/GBP).

In this section, the proposed forecasting method using chaos theory, the embedding theorem, residual analysis and improved ensemble neural networks with genetic algorithms and Taguchi's design of experiments is applied to daily exchange rates between the Euro and the US dollar (EUR/USD) and the US dollar and the Japanese yen (USD/JPY). 


\subsubsection{EUR/USD Exchange Rates Data}

The daily exchange rates between the Euro and the US dollar are selected to evaluate the performance of the proposed forecasting method in the current study. To compare the performance of the proposed forecasting method in this study when applied to EUR/USD exchange rates with the results reported in the literature, the conditions are set based on Sheikhan and Movaghar [73]. The daily exchange rates between the Euro and the US dollar for the period February 01, 2002 to October 31, 2008 (1757 data) as shown in Figure 7.14 is selected for training and testing of the developed model in this study. The first 1600 data are selected for training and the last 157 data are used to test the performance of the proposed forecasting method.

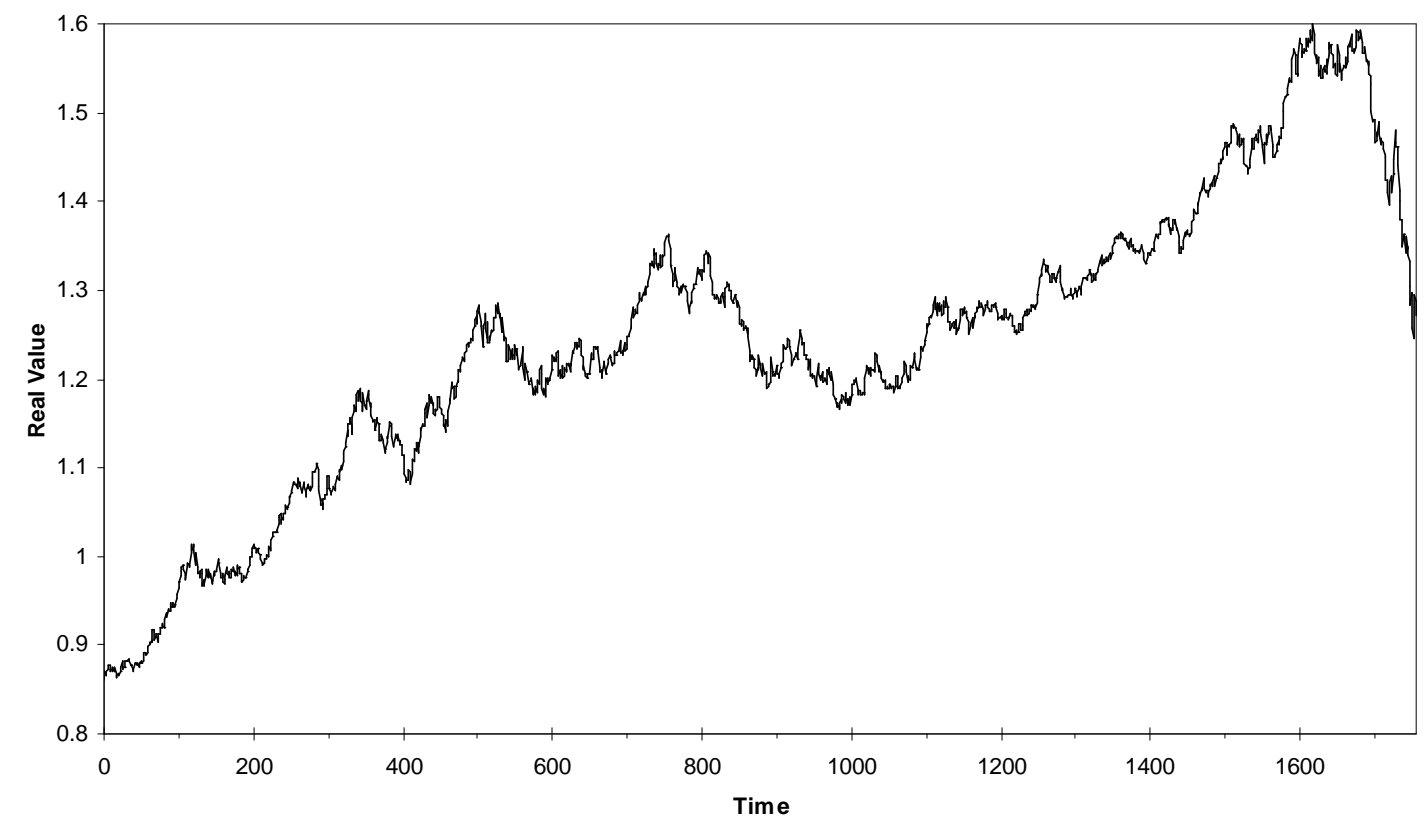

Figure 7.14 The exchange rate EUR/USD from February 01, 2002 to October 31, 2008.

The first step in applying the proposed forecasting method to EUR/USD is to detect chaos in the time series. As explained in Section 3.2.2, a positive Lyapunov exponent confirms the existence of chaos. Figure 7.15 shows prediction error versus length of 
prediction for EUR/USD data. The slope of the linear portion of the graph estimates the value of the largest Lyapunov exponent (LLE). The largest Lyapunov exponent for EUR/USD data is positive, $\operatorname{LLE}=4.54$, which is an indication of chaotic behaviour in the data.

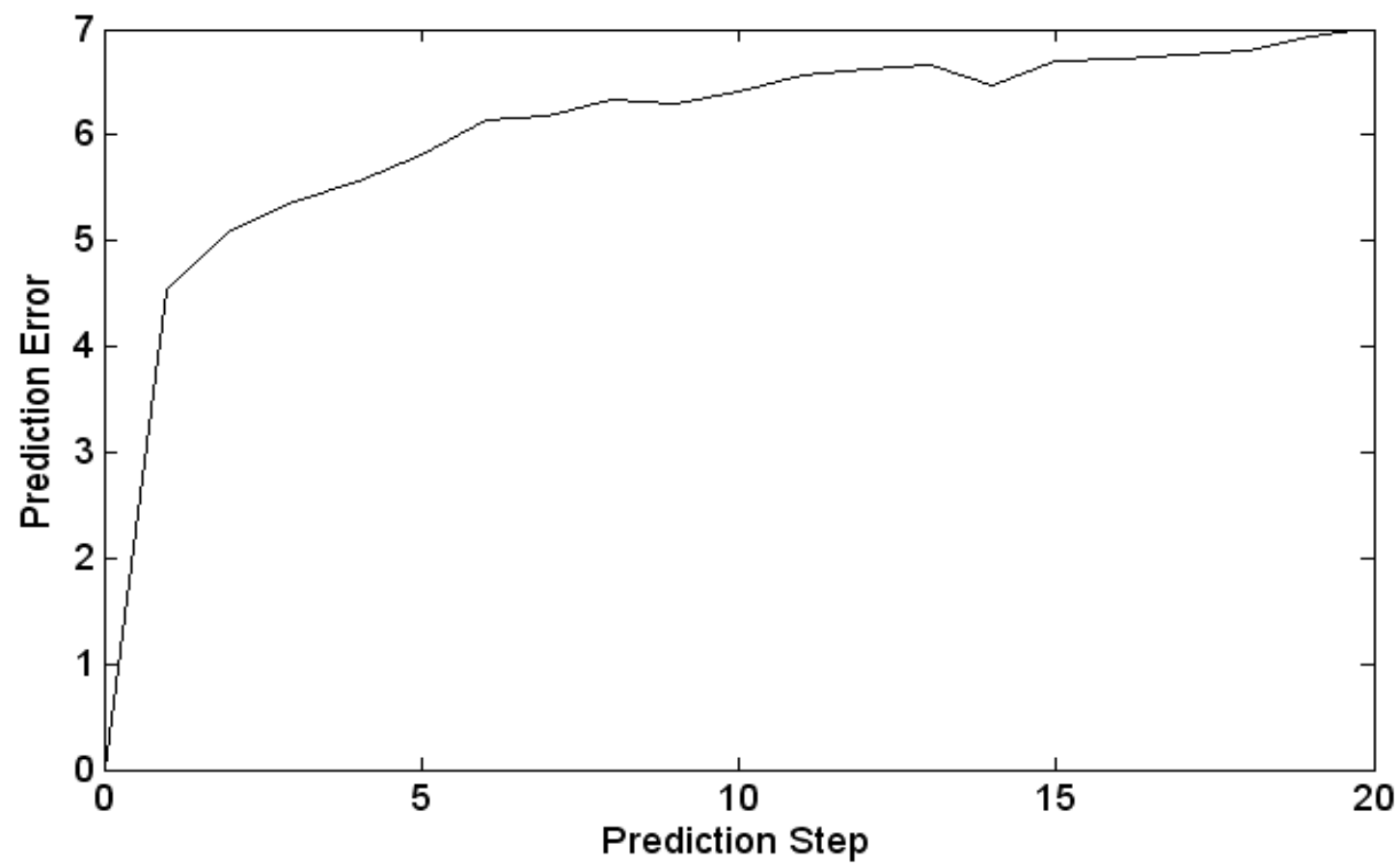

Figure 7.15 Largest Lyapunov exponent graph for EUR/USD data.

The embedding dimension $D$ and the time delay $T$ are calculated using TSTOOL [190]. EUR/USD data is reconstructed into phase space points with embedding dimension $D=4$ and time delay $T=1$. The embedded phase space points are fed into an Elman neural network with 4 neurons in the input and output layers and 6 hidden and recurrent neurons. The weights and biases of the trained Elman neural network are kept to forecast the future values of the phase space points and eventually the future values of EUR/USD data.

The prediction errors for the training data are calculated and analyzed. The largest Lyapunov exponent, $\mathrm{LLE}=8.86$, is determined for the residual time series using TSTOOL [190], which shows the existence of chaos in the obtained residuals. The residuals obtained 
from the original prediction of the training data are reconstructed using the estimated embedding dimension $D_{1}=4$ and time delay $T_{1}=1$. The embedded phase space points are fed into a new Elman neural network. The neural network is set up with 3 neurons in the input and output layers and 6 neurons in the hidden layer and 6 recurrent neurons. The residual analysis is repeated one more time. The largest Lyapunov exponent, LLE $=9.11$, is determined for the second level residuals, which shows the existence of chaos in the obtained residuals. After confirmation that the residuals are chaotic, with $D_{2}=4$ and time delay $T_{2}=$ 1, the second order residuals are reconstructed and predicted using an Elman neural network.

Finally, a NARX neural network is trained using the neural networks' result values of the time series and the residuals as input and the original time series as output. The weights and biases of the final neural network are improved using genetic algorithms and kept to predict the future value of the time series. Figure 7.16 compares the original values of EUR/USD data time series with their predicted values.

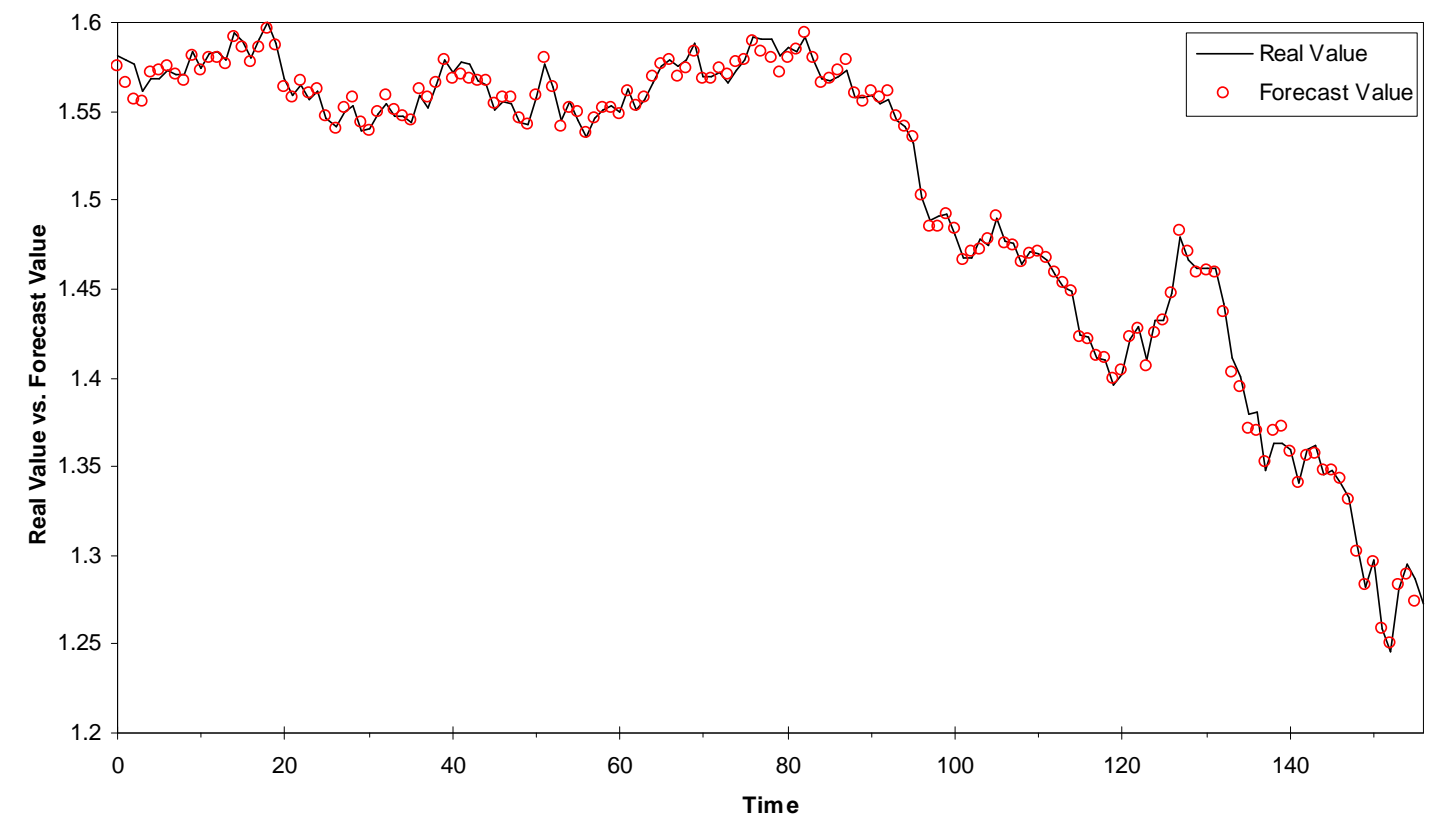

Figure 7.16 The comparison between original EUR/USD data time series and the final predicted values. 
The error indices, MSE and NMSE for the 157 EUR/USD test samples are compared with the results reported in the literature and presented in Table 7.5. The experiment performed for the daily exchange rates between the Euro and the US dollar confirms that the proposed method with improved Elman-NARX configuration has better performance compared to other prediction methods reported in the literature.

Table 7.5 Comparison between the prediction errors reported in the literature and the proposed method (157 EUR/USD test data).

\begin{tabular}{llll}
\hline & & \multicolumn{2}{c}{ Prediction Error } \\
\hline Prediction Method & Reference & MSE & NMSE \\
\hline Evolutionary Connectionist Model & {$[73]$} & $3.10 \mathrm{E}-03$ & $5.00 \mathrm{E}-02$ \\
The Proposed Method - Taguchi's DOE -- & $1.43 \mathrm{E}-04$ & $1.83 \mathrm{E}-02$ \\
\hline
\end{tabular}

\subsubsection{USD/JPY Exchange Rates}

The daily exchange rates between the US dollar and the Japanese yen are selected for analysis and forecasting. In order to compare the performance of the proposed forecasting method in this study when applied to USD/JPY data with the results reported in the literature, the conditions are set based on Liu [255]. The daily exchange rates between the US dollar and the Japanese yen for the period January 01, 2003 and December 31, 2006 (1040 data) as shown in Figure 7.17 are selected for training and testing of the developed model in this study. The first 540 data are selected for training and the last 500 data are used to test the performance of the proposed forecasting method.

The largest Lyapunov exponent for USD/JPY data is positive, LLE $=5.22$, which is an indication of chaotic behaviour in the data. The embedding dimensions $D=3$ and $T=5$ are estimated as explained in Section 3.2.4 and 3.2.5 using TSTOOL [190]. Accordingly, 
phase space points are reconstructed. Figure 7.18 shows USD/JPY data in the phase space domain with $D=3$ and $T=5$.

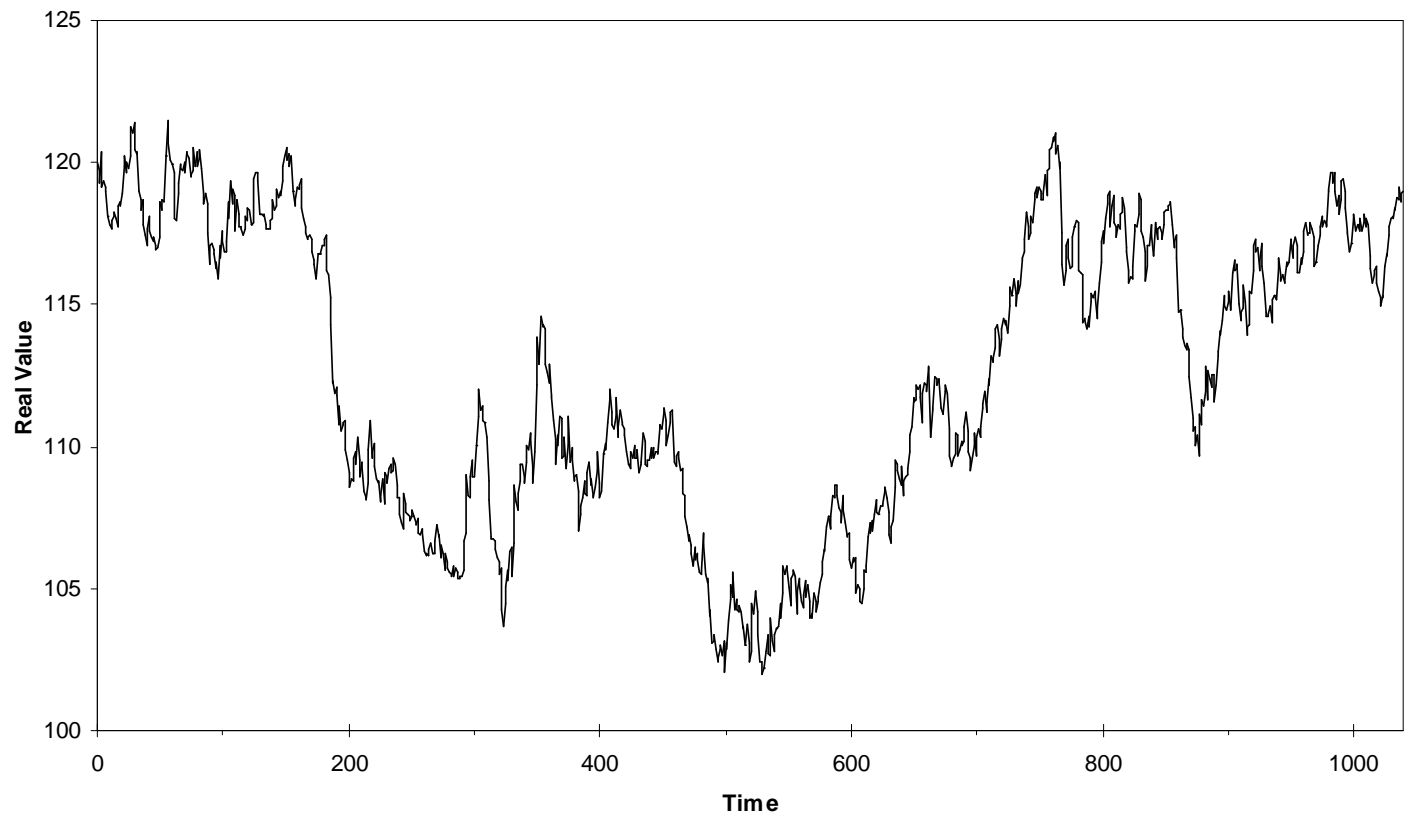

Figure 7.17 The daily exchange rates between the US dollar and the Japanese yen for the period January 01, 2003 and December 31, 2006.

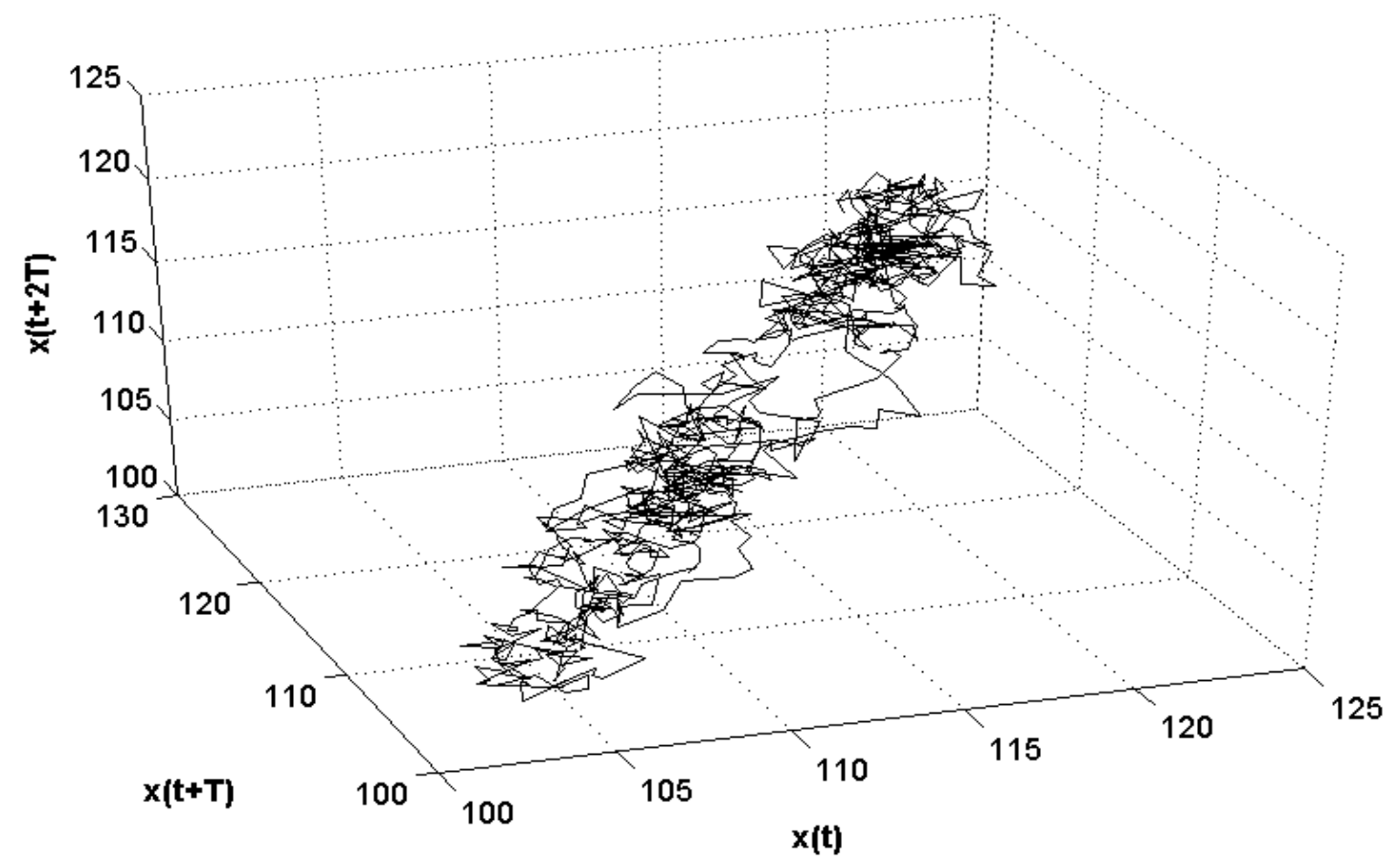

Figure 7.18 USD/JPY data in the phase space domain with $D=3$ and $T=5$. 
An Elman neural network is structured with 3 neurons in the input layer, 6 neurons in the hidden layer, 6 recurrent neurons and 3 neurons in the output layer and then trained. The weights and biases of the network are kept for prediction purposes. Figure 7.19 compares the real data with the predicated values for 94 data kept for testing.

The prediction errors for the data selected for the training are calculated and analyzed. The largest Lyapunov exponent for the residual time series, LLE $=6.76$, is positive; therefore, it is concluded that the residuals exhibit chaotic behaviour. Based on this finding, the residuals are treated as a new chaotic time series and the prediction method proposed in Section 5.1 is applied to predict the future value of the residuals. The first order residuals are reconstructed with embedding dimension $D_{1}=3$ and time delay $T_{1}=1$. Figure 7.20 presents the residuals of the original prediction and the predicted values for the test data.

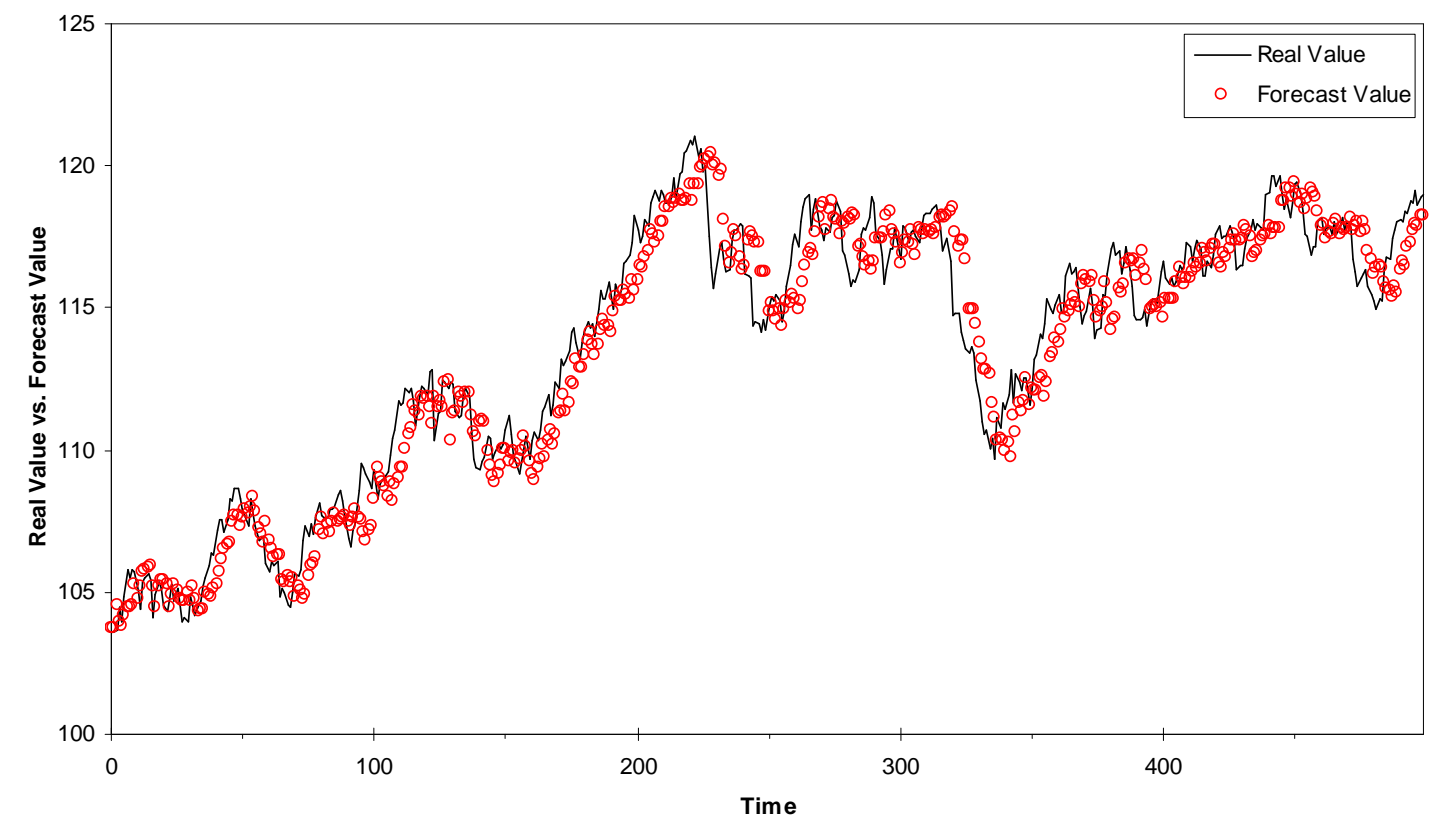

Figure 7.19 USD/JPY data - Comparing the original test data and the predicted values. 


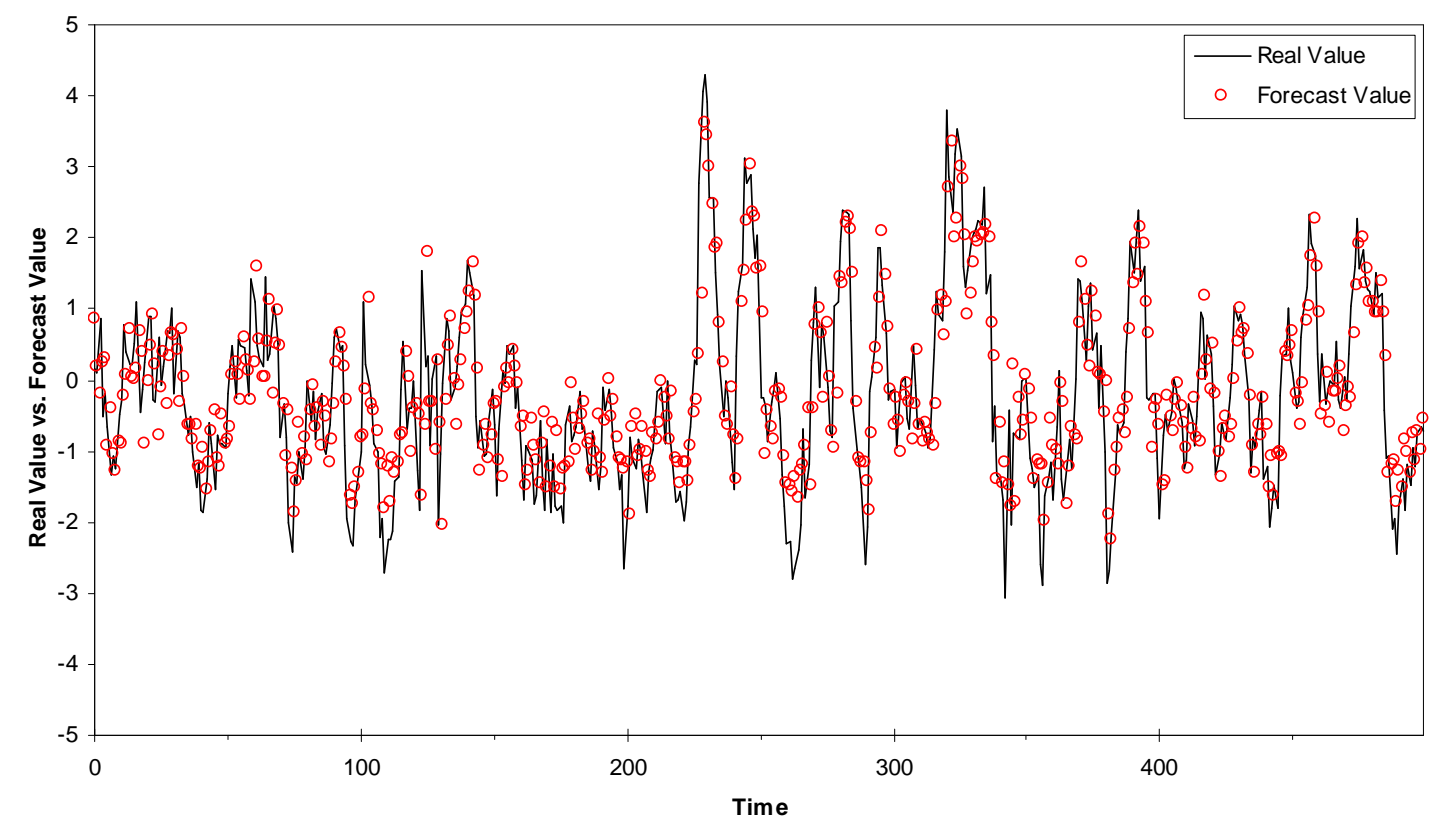

Figure 7.20 USD/JPY data - The comparison of the first residual values and the predicted values.

The residual analysis is repeated one more time. The largest Lyapunov exponent for the second level residuals, LLE $=8.20$, is determined using TSTOOL [190], which shows the existence of chaos in the obtained residuals. After confirmation that the residuals are chaotic, with $D_{2}=3$ and time delay $T_{2}=1$, the second order residuals are reconstructed and predicted using an Elman neural network. Finally, a NARX neural network is trained using the neural networks' result value of the time series and the residuals as input and the original time series as output. The NARX neural network is used to capture the relationship between the predicted values and the original time series. The weights and biases of the final neural network are improved using genetic algorithms and kept to predict the future value of the time series. Figure 7.21 compares the original values of USD/JPY data time series with their predicted values.

In order to evaluate the prediction performance and compare it with the results reported by Liu [255], RMSE is calculated. The prediction accuracy for 500 USD/JPY 
test data, for the demonstrated experiment based on the improved Elman-NARX configuration are compared with the results reported in the literature.

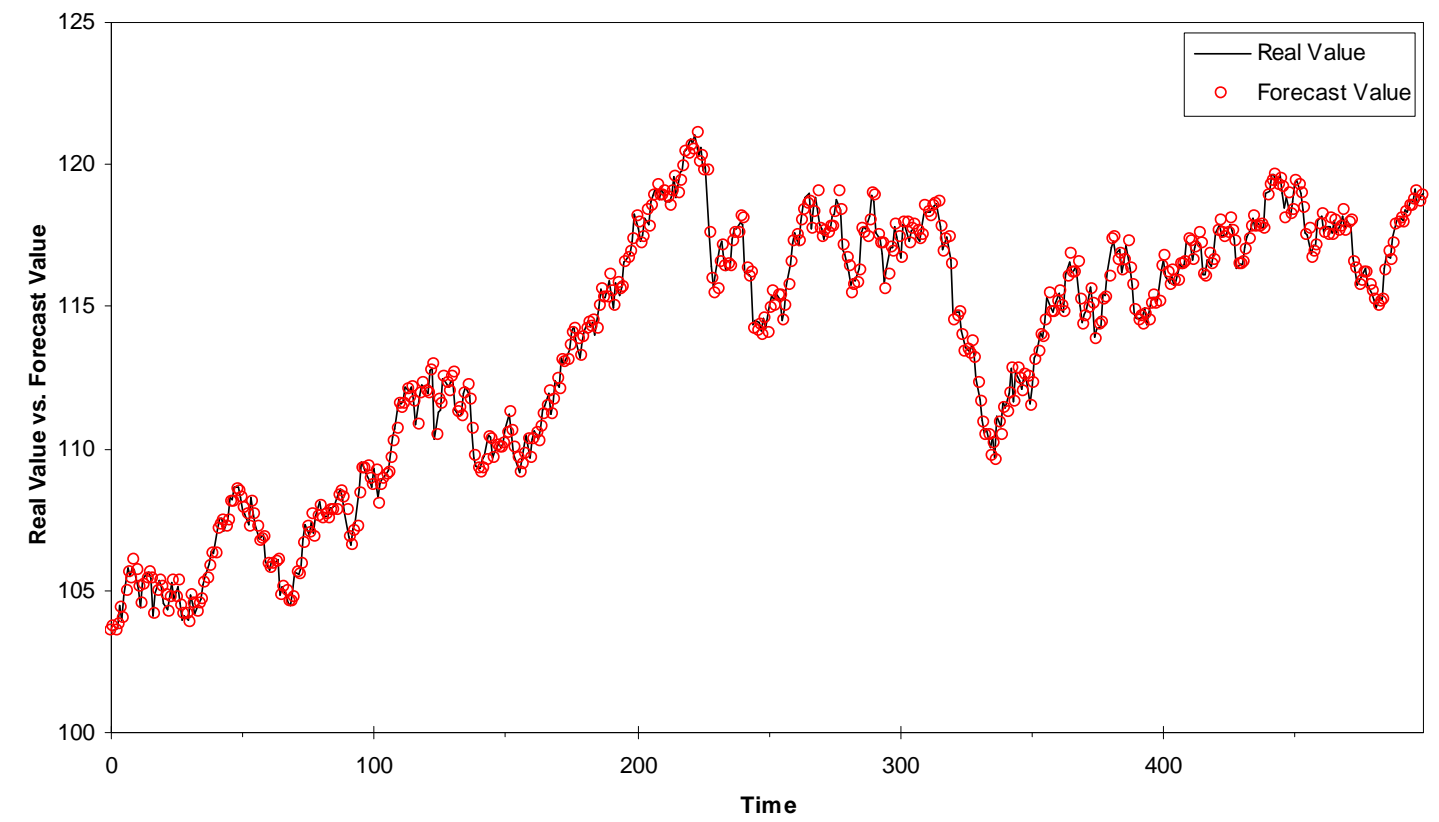

Figure 7.21 The comparison between original USD/JPY data and the final predicted values.

Table 7.6 presents the comparison between the prediction errors, RMSE reported in the literature and the proposed method for USD/JPY data. This experiment confirms that the proposed method with improved Elman-NARX, when it is used to forecast the future values of USD/JPY data, generates better results compared to other prediction methods reported in the literature.

Table 7.6 Comparison between the prediction errors reported in the literature and the proposed method (500 USD/JPY test data).

Prediction Error

\begin{tabular}{lll}
\hline Prediction Method & Reference & RMSE \\
\hline Dynamic Adaptive Neuron-Fuzzy Logic & {$[255]$} & 2.55 \\
The Proposed Method - Taguchi's DOE & -- & $5.96 \mathrm{E}-01$ \\
\hline
\end{tabular}




\subsection{Summary}

The application of chaos theory is increasing in various disciplines. Chaos has been observed in mixing processes, the control of robot servos, communications, industry, economics, ecology, international relations, medicine, supply chain management, inventory management, cardiovascular control system, hydrological field, operations research and financial markets.

A robust technique to predict chaotic systems can increase the efficiency of the system under study and decrease the risk. Chaotic system forecasting has been reported in many areas such as financial markets, supply chain management, oil price and consumption, power load, production systems and others. In financial markets, chaos theory has been applied to commodity markets, stock markets and exchange rates among others. The stock markets and foreign exchange markets are vital parts of the global financial markets. The financial researchers closely studied the stock markets and foreign exchange markets and observed the evidence of complexity, nonlinearity and dynamical behaviour in both markets.

In this chapter, the forecasting method developed with the residual analysis method using ensemble neural networks and improved by genetic algorithms and Taguchi's design of experiments was applied to financial markets, namely, stock markets and exchange rate trading. The daily price of four stocks, namely, British Airlines, Southwest Airlines, Ryanair Holdings and Lucent Incorporated and two exchange rates, namely, EUR/USD and USD/JPY were analyzed and their future values were predicted. In all the numerical experiments, the proposed forecasting method shows better performance compared to the results reported in the literature. 


\section{CHAPTER 8}

\section{CONCLUSION AND FUTURE WORK}

\subsection{Conclusion}

Chaos theory has been used to study and analyze chaotic systems. Chaotic systems generate random-look data; however, in the embedding phase space, they present deterministic behaviour. Forecasting chaotic time series has increasingly become a popular and challenging subject. Many of the forecasting methods proposed in the literature are either inefficient when applied to chaotic time series, or difficult to implement. The motivation to conduct the current study was to develop a more effective, easy-to-use and practical method to forecast chaotic time series.

In this study, an original technique was developed to forecast one step ahead of a given chaotic time series. The key difference between this technique and the techniques proposed in the literature was the utilization of the precise definition of chaos theory and the embedding theorem. Chaos theory states that, for a chaotic system, there exists an unknown mathematical equation which can describe the system. Also, the embedding theorem claims that in phase space mode, a chaotic system reflects most of the characteristics of the original system. Therefore, the unknown state of the given chaotic system can be replaced by the generated phase space points. Also, the unknown function which governs the original system can be estimated with a neural network. In the current technique, an artificial neural network was trained using a single phase space point 
(current state) as input and a single phase space point (next state) as output. This complies precisely with the definition of chaos theory and the embedding theorem.

The first step to proceed with the proposed forecasting method was to determine the existence of chaos in a given time series. When the presence of chaos was detected in the observed time series, then the phase space parameters were calculated and the phase space points were generated. An artificial neural network with a single phase space point, $D$ input units, and a single phase space point, $D$ output units, was trained. After training the artificial neural network, the weights and biases of the network were kept to forecast the unknown phase space points and obtain the future values of chaotic time series.

In order to evaluate the performance of the proposed method, the developed technique was applied to three chaotic time series: Mackey-Glass, Logistic and Henon. The feedforward neural network and the Elman recurrent neural network were used to estimate the unknown chaotic function. In most training algorithms, the initial weights and biases of the neural network are selected randomly. This can cause variation in the output of the neural network. To reduce the variation of the neural network output and select proper initial weights and biases for the neural network, genetic algorithms were adopted. The GA's objective function was to minimize the mean square error (MSE) of the predicted values based on the proposed technique and the original time series. The decision variables were weights and biases of the neural network. When the GA was applied, the best weights and biases which improved the performance of the neural network were selected. The combination of the proposed forecasting method along with genetic algorithms presented an outstanding result. The comparison between the result of the proposed technique and the results reported in the literature substantiates the effectiveness of the proposed method. 
Through an extensive analysis, it was observed that, in some cases, heuristic models could not completely capture the characteristics of the original time series and, therefore, the residuals became highly correlated. The residuals resulting from the developed neural network prediction method were further analyzed, and it was observed that, in some cases, they demonstrate chaotic behaviour.

To investigate the contribution of the residual analysis in improving the performance of the proposed forecasting method, the obtained residuals were considered as a new chaotic time series and analyzed separately. When the presence of chaos was confirmed in the residual time series, the embedding dimension and the time delay were determined and the phase space points were generated. A new artificial neural network, with $D_{1}$ input units and $D_{1}$ output units, was trained and the future values of the residuals were obtained.

To correlate the neural networks' result values of the time series and the residuals to the original time series, a new neural network was trained. The neural network with two input units and one output unit was trained. The neural networks' result values of the time series and the residuals were used as input and the original time series as output. To increase the accuracy of the prediction, the residual analysis could be repeated several times. To correlate the neural networks' result values of the time series and the residuals in various levels to the original time series, a neural network with $M+1$ input units, where $M$ is the level of residual analysis, and one output unit, could be trained. As before, the proposed method was merged with genetic algorithms to improve the forecasting results.

To examine the performance of the proposed method, a feedforward-feedforward configuration was applied to two simulated chaotic systems: Mackey-Glass and Lorenz equations. Also, an Elman-NARX configuration was applied to the Mackey-Glass and 
Lorenz equations and to one real life time series, sunspot time series. Despite its simplicity in implementation, the proposed model exhibited a superior performance in prediction of chaotic time series when compared with the results reported in the literature.

Taguchi's design of experiments as an efficient approach to identify appropriate factor-level combinations was utilized to improve the result of the proposed forecasting method. A systematic approach was introduced using Taguchi's design of experiments to improve the combination selection of ensemble artificial neural networks and their parameters. The method was applied to two benchmarking time series, the Mackey-Glass and Lorenz, and two real life chaotic time series, namely, sunspot and far-infrared NH3 laser time series. The conclusion of Taguchi's DOE for all four time series revealed that among eight selected factors, Tier Neural Network Architecture, Final Neural Network Architecture, Tier Network Training Method and Final Network Training Method showed importance and their selected levels were Elman, NARX, traingdx and trainlm. The experimental results confirmed that the selected levels identified by Taguchi's DOE improved the performance of the proposed forecasting method when compared with the same method using arbitrary selection of artificial neural network architectures and parameters or other existing forecasting methods in the literature.

The proposed forecasting method was applied to financial markets, namely, stock markets and exchange rate. The daily price of British Airlines, Southwest Airlines, Ryanair Holdings and Lucent Inc. and two exchange rates, namely, EUR/USD and USD/JPY were analyzed and predicted. In all the numerical experiments, the proposed forecasting method based on improved Elman-NARX configuration using Taguchi's DOE and GA showed better performance compared to the results reported in the literature. 


\subsection{Contributions}

The main objective of this research work was to develop an easy-to-use, effective and practical method to forecast chaotic time series. This goal was achieved by proposing an easy-to-use forecasting method which exhibits superior performance when compared with other forecasting methods in the literature.

The following represents the contribution of this research work:

- After extensive review of the literature, it was noted that none of the existing approaches makes full use of the precise definition of chaos theory, which requires a single phase space point as input and a single phase space point as output. Therefore, the precise definition of chaos theory and the embedding theorem was adopted to develop an effective forecasting method. This version of the proposed forecasting method showed better performance when compared with similar methods in the literature.

- A thorough analysis of residuals was conducted to investigate the characteristics of the residuals. The investigation demonstrated that chaos was present in some of the test problems. Therefore, the method was further enhanced by incorporating the residual analysis into the proposed forecasting method.

- Several neural network architectures were utilized in this study, and the impact of the various architectures on the efficiency and effectiveness of the proposed forecasting method was examined. The main application of artificial neural networks is to estimate the unknown underlying function governing the system under study and capture the relationships among the given data. Feedforward neural networks from a static category and Elman and NARX neural networks 
from a dynamic category were selected for the analysis. It was concluded that the dynamic architectures in the proposed configuration perform better compared to the static neural networks.

- In this study, genetic algorithms have been adopted to select the initial weights and biases of artificial neural networks to improve the performance of the networks. The numerical examples substantiated that genetic algorithms can enhance the effectiveness of the proposed forecasting method by selecting proper initial weights and biases for the selected artificial neural networks.

- Several configurations and parameters are involved in the structure of the proposed forecasting method. Design of experiments (DOE) is a well-known method to select a proper combination of the parameters to optimize the result. Taguchi's DOE, as an efficient method in selecting appropriate factor-level combination was adopted to improve the performance of the proposed method. A systematic approach based on Taguchi's DOE was introduced to facilitate the combination selection of the ensemble artificial neural networks and their parameters. The numerical examples demonstrated that the improved forecasting method using Taguchi's DOE performs better when compared with the same method using an arbitrary selection of artificial neural network architectures and parameters or other existing forecasting methods in the literature.

- Several benchmarking and a couple of real life chaotic time series are selected for numerical experiments. An extensive literature review was performed to compare the performance of the proposed forecasting method with the various forecasting models in the literature. The comparison between the results obtained in this study 
and the results reported in the literature established the superiority and effectiveness of the proposed forecasting method.

- Stock markets and exchange rate markets are the essential and important parts of the global economics. The existence of chaos has been observed in both markets. Forecasting the future of these markets can facilitate planning and decision making in these areas and reduce the associated risks. The forecasting method proposed in this research was applied to both stock markets and foreign exchange rates. The obtained results confirmed that the proposed forecasting method is an easy-to-use, effective and practical method to forecast the future of stock markets and the exchange rate markets.

\subsection{Future work}

This dissertation presented an original method to forecast chaotic time series more effectively and accurately. The results of the numerical experiments confirmed that the proposed method performed better than other forecasting models in the literature in the investigated areas. However, to further improve the quality of the current study, the followings can be investigated as an extension to this research work.

- In this study, only two levels of residual analysis were examined. In all examined numerical experiments, two levels of residual analysis delivered better performance when compared with the results presented in the literature. In future work, this can be extended to more levels of residual analysis to increase the accuracy of forecasting. 
- Application of chaos theory has been observed in many fields such as communications, ecology, international relations, medicine, hydrological field, supply chain management and financial markets. In this study, the proposed forecasting method was applied to some benchmarking and real life chaotic time series. Also, in financial section, the developed forecasting method was applied to stock markets and foreign exchange rates. This can be extended in future work to other fields such as supply chain management, product design, inventory management and demand forecasting.

- There are several factors and parameters involved in the design of the proposed forecasting method. These factors consist of the neural network architecture, neural network parameters, embedding parameters and genetic algorithm parameters. In the current study, Taguchi's design of experiments was employed to improve the performance of the proposed forecasting method by selecting the appropriate level of the involved factors. Among all the involved factors and parameters, only the impact of the neural network architecture, transfer function, and training method in two levels were studied in this research. The impact of other factors can be investigated and more levels can be selected for the factors.

- The forecasting method developed in this study has been applied to stock market and exchange rate data. In future work, the proposed forecasting method can be utilized in loss and profit analysis and portfolio management.

- In the current study, the number of training and test data are selected according to the literature to be able to compare the performance of the proposed method with the results reported in the literature. In future work, the optimum proportion of 
training and test data can be determined. Also, in the proposed method, the trained neural network is used to forecast all the test data. An investigation can be conducted to determine when the forecasting performance drops and the neural networks require re-training.

- The validity of the proposed can be tested on other types of time series such as deterministic, stationary, non-stationary, seasonal, stochastic and other.

- In the current study, only univariate chaotic time series are analyzed. The application of the proposed forecasting method can be extended to multivariate chaotic time series.

- The current research work is applied to forecast one step ahead of chaotic time series. The performance of the proposed forecasting method for long term prediction of chaotic time series can be investigated. 


\section{Appendix A - A Numerical Example for Chaos Theory and Embedding Theorem}

As a numerical example to explain chaos theory and Taken's embedding theorem, the Lorenz time series is analyzed. The Lorenz system is a simplified version of NavierStokes equations which are used in fluid mechanics. Based on Sprott [171], the original state of the Lorenz system is defined in a 3-dimensional space. The states of the Lorenz system are $s_{1}$, "the speed of rotation", $s_{2}$, "the temperature difference between the rising and falling fluids" and $s_{3}$, "the distortion from linearity of the vertical temperature profile" [171]. The original state of Lorenz system can be described as $s(t)=\left[s_{1}(t), s_{2}(t)\right.$, $\left.s_{3}(t)\right]$. Figure A.1 exhibits the original states of Lorenz system, $s(t)$.

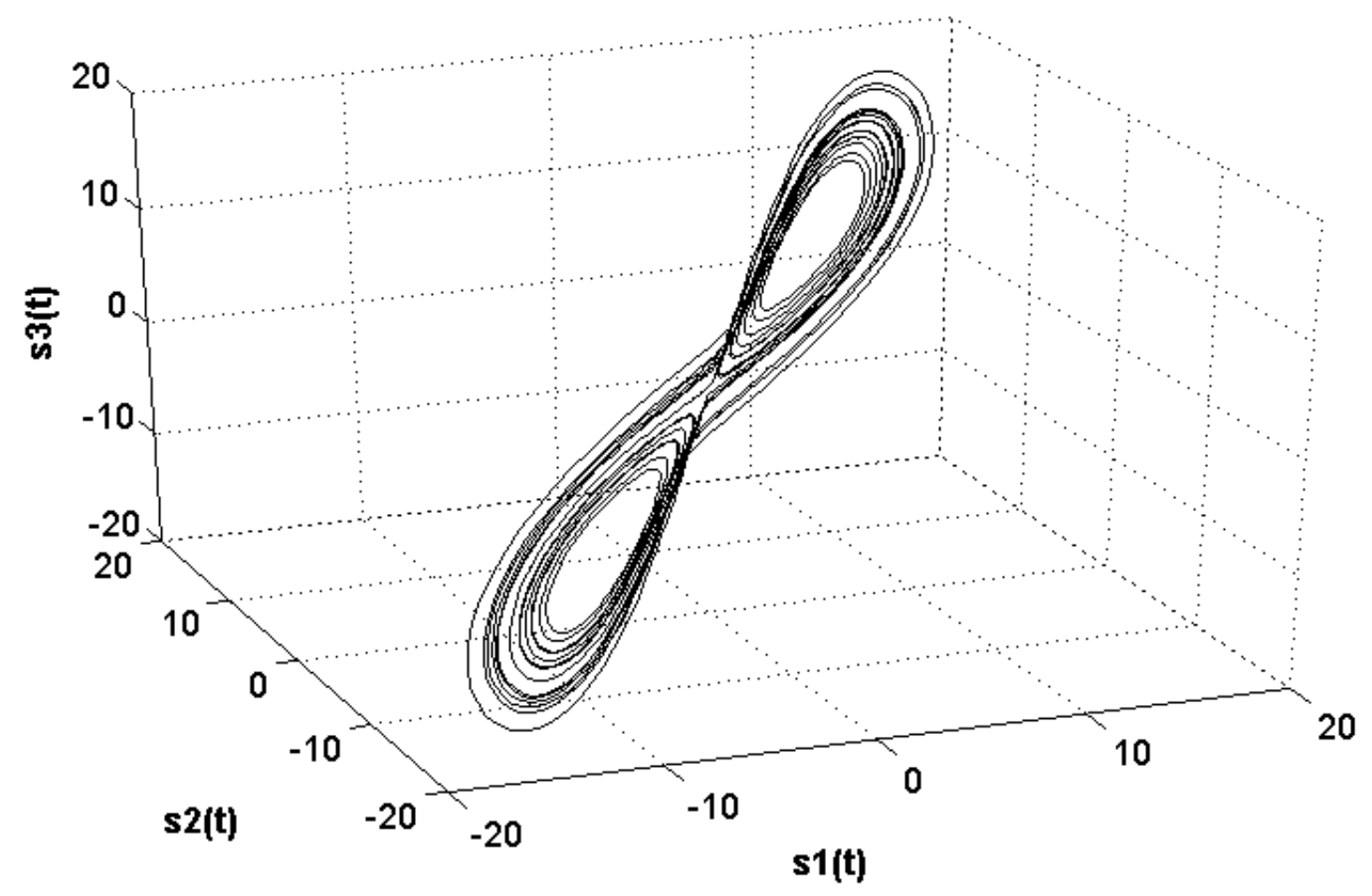

Figure A.1 The original states of Lorenz system. 
In real life, the state of a chaotic time series, $s(t)$, is not known. Although chaotic systems are deterministic, we may not be able to determine the governing equations. However, we can observe or measure some scalar values from a chaotic system. Chaos theory and Takens' embedding theorem claim that these scalar values, $x(t)$, can reconstruct the state of the original system, $s(t)$, if the time delay $T$ and the embedding dimension $D$ are properly determined [20].

Based on chaos theory and Taken's embedding theorem, if a single measurement such as the temperature difference is observed from the Lorenz system, then with the time delay and the embedding dimension, the observed time series can be embedded in a phase space which reflects the characteristics of the original system, $s(t)$. Now it is required to determine the time delay $T$ and the embedding dimension $D$ using the methods explained in Sections 3.2.4 and 3.2.5. The time delay and the embedding dimension for the Lorenz system are determined, $T=3$ and $D=3$. Based on the conditions set in Section 5.2.2, the first 11 data points $\{x(1), x(2), \ldots, x(11)\}$ from the Lorenz time series are $\{8.6927,9.2594$, $9.8433,10.4384,11.0369,11.6293,12.2042,12.7485,13.2480,13.6873,14.0513\}$. Based on Section 3.2.3, the general phase space point for a system with $T=3$ and $D=3$ will be $Y(t)=[x(t), x(t+T), x(t+2 T)]$. Accordingly, $Y(1)=[x(1), x(4), x(7)]$ or $Y(1)=[8.6927$, $10.4384,12.2042]$ and similarly $Y(2)=[9.2594,11.0369,12.7485], Y(3)=[9.8433$, $11.6293,13.2480], Y(4)=[10.4384,12.2042,13.6873]$, and $Y(5)=[11.0369,12.7485$, 14.0513]. Figure A.2 shows the attractor of the observed samples when reconstructed into the phase space points. Comparing Figures A.1 and A.2, it can be concluded that the embedding phase space points in Figure A.2 reflect the characteristics of the Lorenz system presented in Figure A.1. 


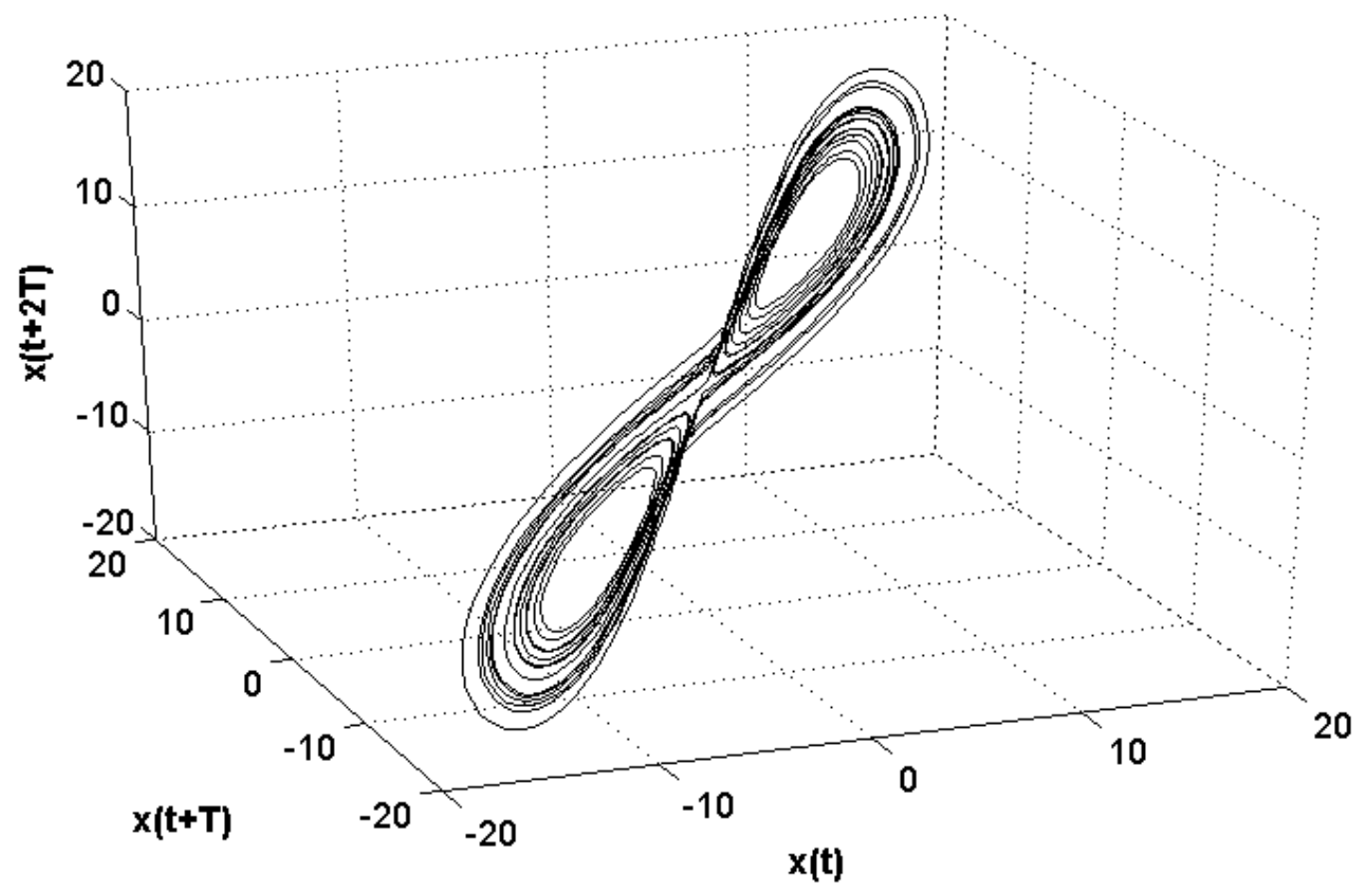

Figure A.2 Lorenz time series reconstructed into the phase space points with $T=3$ and $D=3$. 


\section{Appendix B - A Numerical Example to Detect the Existence of Chaos}

Four methods are introduced in Section 3.2.2 to determine the presence of chaos in a given time series. In this section, the Henon time series which has been used in the literature as a benchmark time series is selected to demonstrate how the existence of chaos can be detected in a given time series. The details of the Henon time series are given in Section 4.2.3. The Henon time series with 1000 data points is shown in Figure B.1.

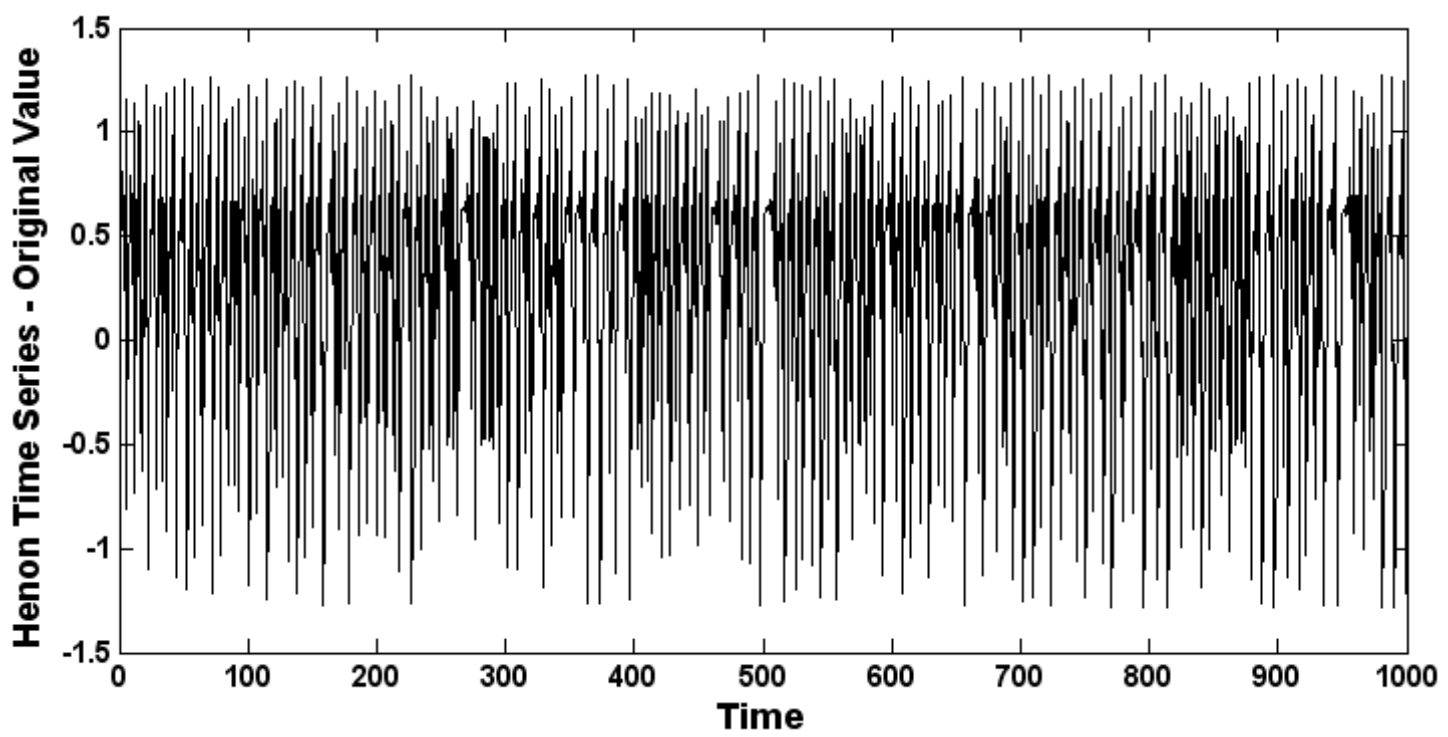

Figure B.1 Henon chaotic time series with 1000 data points.

The first method which is utilized to investigate the existence of chaos is Fourier transform or a power spectrum plot. As highlighted in Section 3.2.2, the spectrum for chaotic time series will be broadband with a broad peak. Figure B.2 is a power spectrum plot for the Henon time series. Figure B.2 confirms the presence of chaos in the Henon time series as the power spectrum is broadband with a broad peak. 


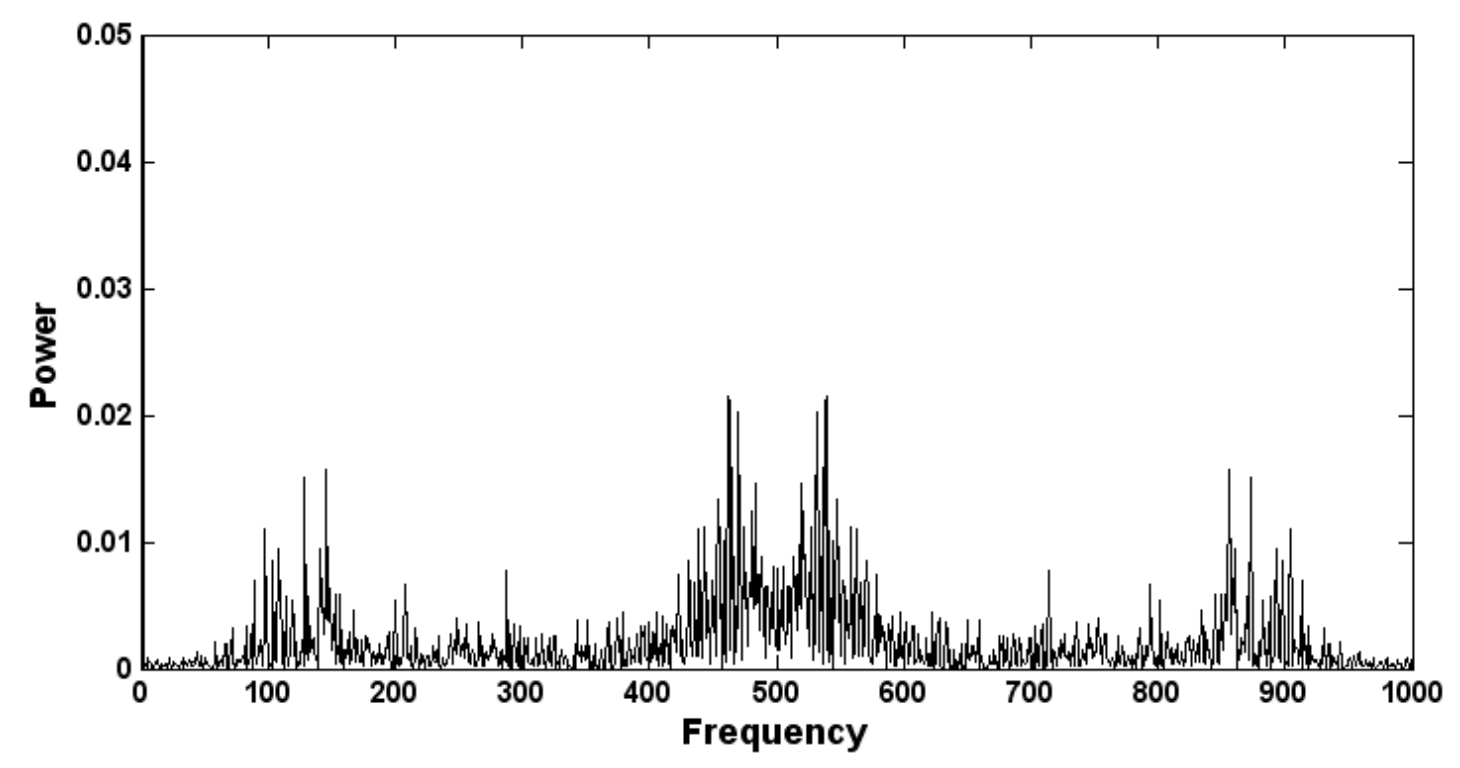

Figure B.2 Power spectrum plot for Henon time series.

The second technique to identify the characteristic of a time series is the Lyapunov exponent method. The slope of the linear portion of the largest Lyapunov exponent graph, prediction error versus length of prediction, gives an estimate of the largest Lyapunov exponent $[10,13]$. The function largelyap in TSTOOL [190], which is an algorithm based on the work by Wolf et al. [161] is used to generate Figure B.3. Figure B.3 shows the largest Lyapunov exponent plot for the Henon time series. The slope of the linear portion of Figure B.3 is 5.74. The positive value of the largest Lyapunov exponent exhibits an exponential divergence of the trajectories and consequently the presence of chaos in the Henon time series.

The next method to identify the nature of a time series is the Hurst exponent. As mentioned in Section 3.2.2, the Hurst exponent values between 0 and 0.5 and 0.5 and 1 show chaotic behaviour. The Hurst value for the Henon time series is calculated based on the formulations in Section 3.2.2. The $H=0.3313$ is determined for the Henon time series which proves the presence of chaos with limited predictability. 


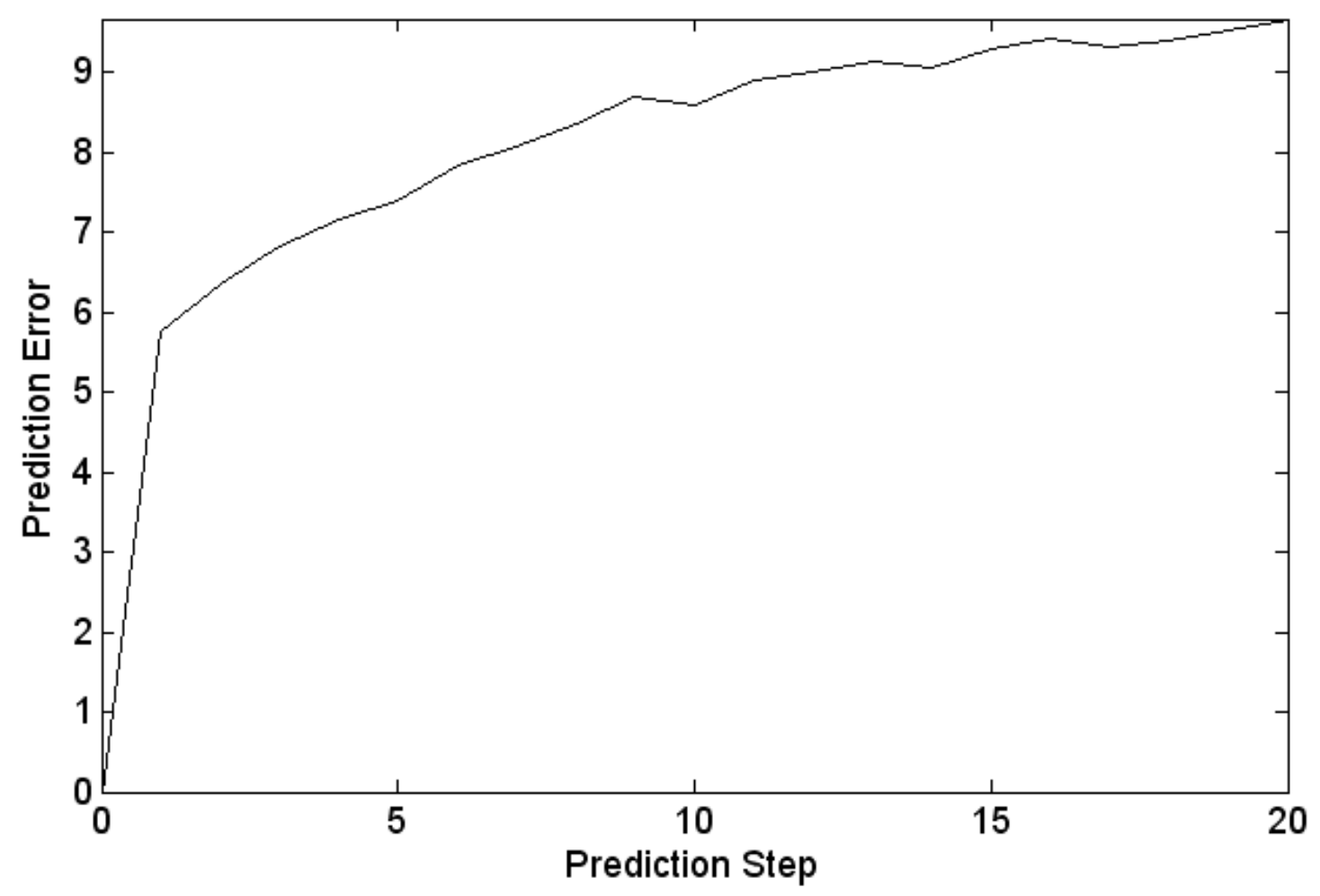

Figure B.3 The largest Lyapunov exponent plot for Henon time series.

The final method to analyze a given time series is fractal dimension. The fractal dimension for the Henon time series is determined using function takens_estimator in TSTOOL [190], which is an algorithm based on the work by Hilborn [175]. The fractal dimension for the Henon time series is 1.2958 which is not an integer value and confirms dynamical behaviour in this time series. 


\section{Appendix C - A Numerical Example to Determine Embedding Parameters}

To use Taken's [20] embedding theorem, it is essential to calculate embedding parameters, namely, time delay and embedding dimension. The formulations presented in Section 3.2.4 and 3.2.5 and TSTOOL [190], an add-on toolbox for MATLAB [191] are utilized to determine $T$ and $D$.

As mentioned in Section 3.2.4, average mutual information is a suitable method to identify the time delay for a given chaotic time series. The function amutual in TSTOOL [190], which is an algorithm based on the work by Abarbanel [62] is used to generate Figure C.1. Time delay for the Henon time series based on the average mutual information graph in Figure C. 1 is $T=1$.

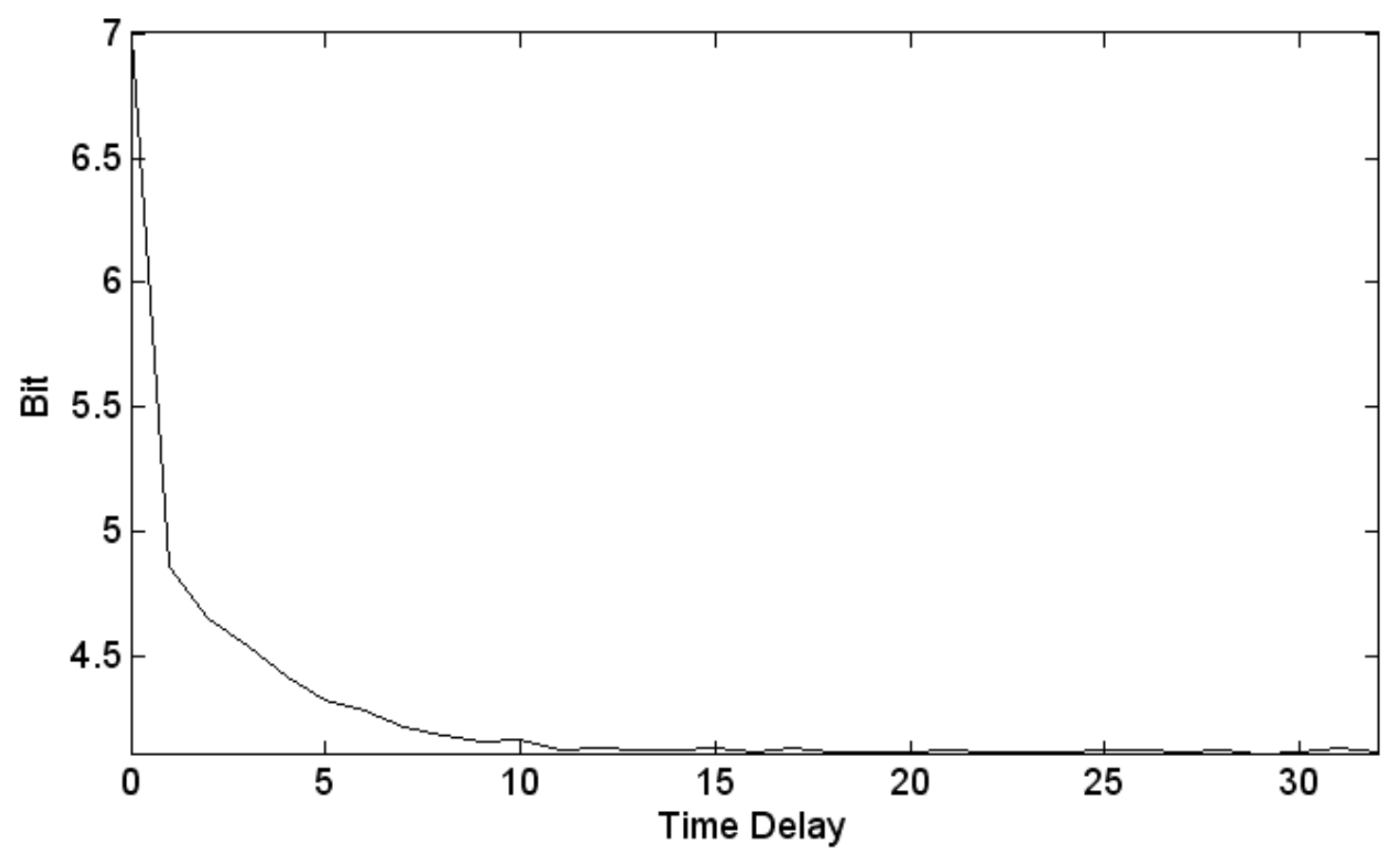

Figure C.1 Average mutual information graph for Henon time series. 
The next step after selecting the time delay is to determine the embedding dimension. The embedding dimension for the Henon time series is determined using function cao in TSTOOL [190], which is an algorithm based on Cao's method [176]. Based on the graph generated using Cao's method shown in Figure C.2, there is a kink at 2. Therefore, for the Henon time series the embedding dimension is $D=2$.

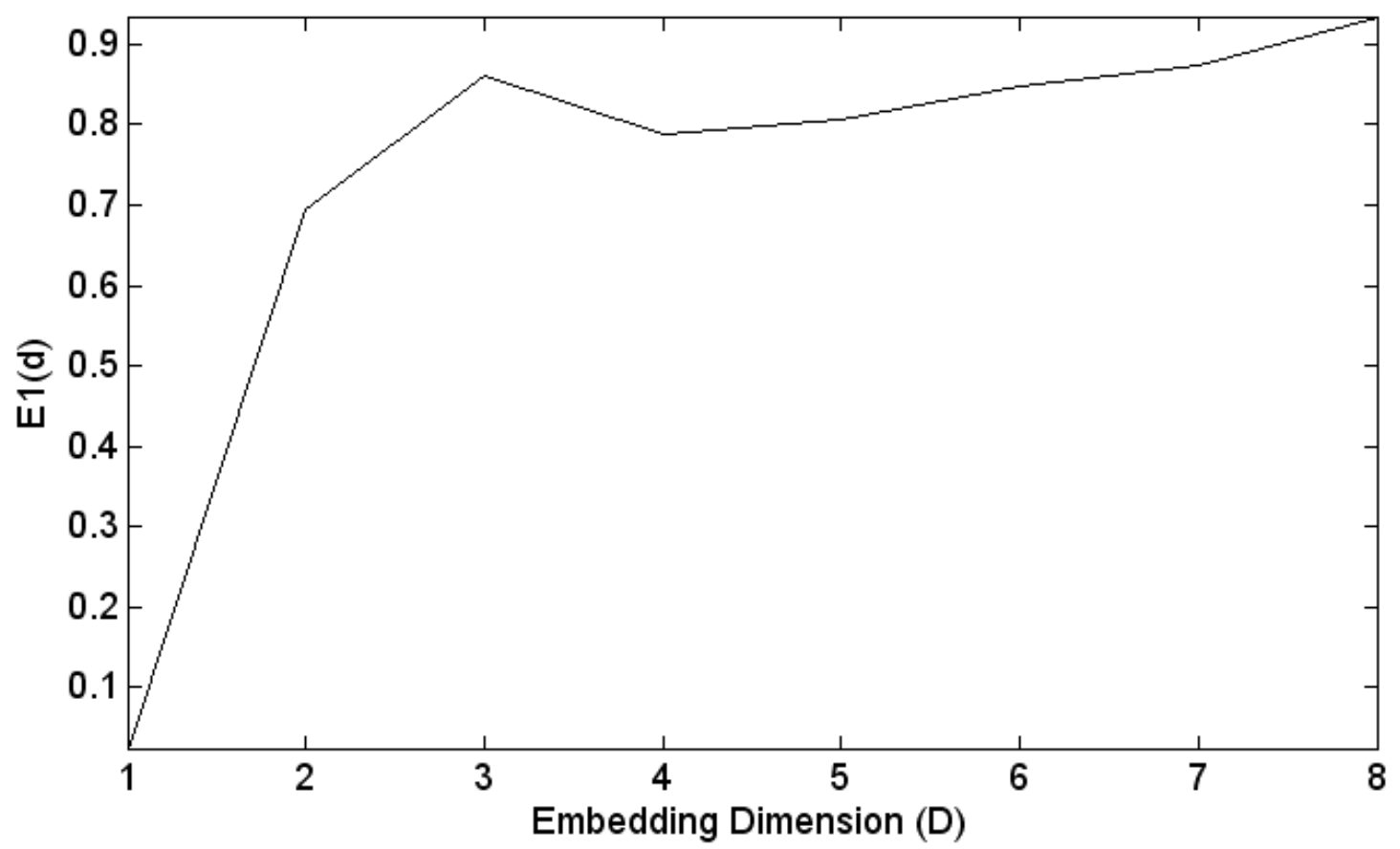

Figure C.2 Cao's graph to identify embedding dimension for Henon time series.

When the presence of chaos is identified in a time series and embedding parameters are determined, the time series is reconstructed into the phase space points. Using time delay $T=1$ and embedding dimension $D=2$, the Henon time series is reconstructed into the phase space points. Figure C. 3 shows the Henon time series in the phase space domain. 


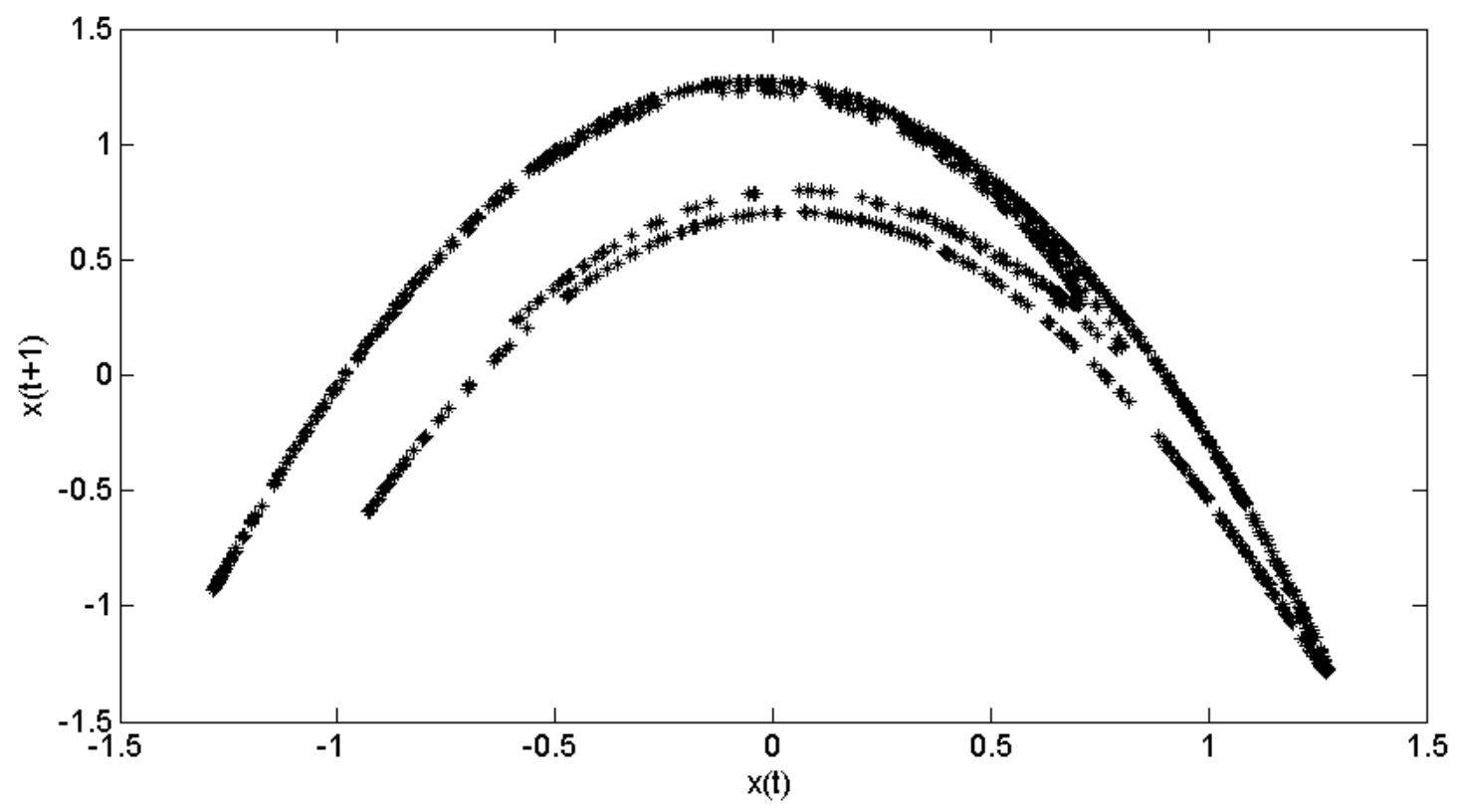

Figure C.3 Henon time series in phase space domain. 


\section{Appendix D - Regression Analysis for Artificial Neural Networks}

This Appendix exhibits the regression analysis between the network response and the corresponding targets for the feedforward and the Elman neural network in the presented numerical analysis in Chapter 4. MATLAB [191] is used to perform the analysis.

Figure D.1 shows the regression analysis between the feedforward network response and the corresponding targets when applied to the Mackey-Glass time series. Correlation coefficient between the outputs and targets ( $R$ value) is 0.9986 which proves a high correlation between the results of the feedforward neural network and the targets.

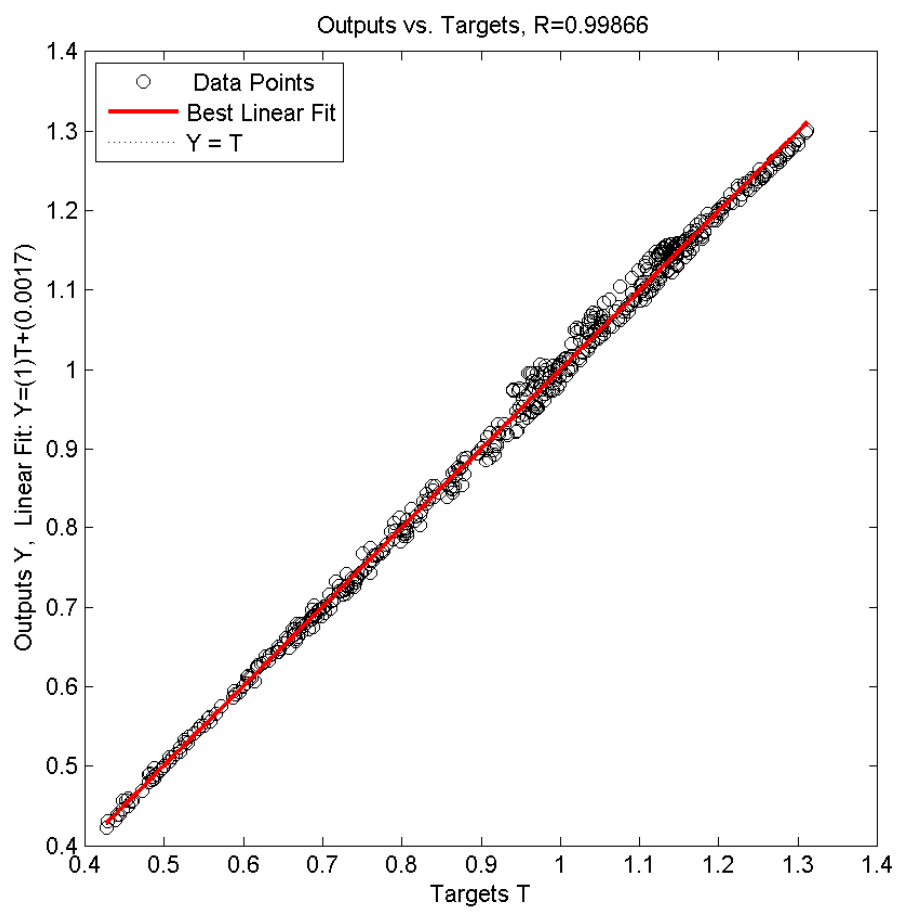

Figure D.1 The regression analysis between the feedforward network response and the corresponding targets when applied to Mackey-Glass time series. 
Table D.1 shows the regression analysis for the Elman and the feedforward neural network responses when applied to the Mackey-Glass, Logistic and Henon time series. In all the analysis, the correlation coefficients ( $\mathrm{R}$ values) show a high correlation between the results of the neural networks and the targets.

Table D.1 The regression analysis between the proposed Elman and feedforward neural network response and the corresponding targets when applied to Mackey-Glass, Logistic and Henon time series.

\begin{tabular}{llll}
\hline & \multicolumn{3}{c}{ Regression Analysis Coefficient } \\
\hline The Proposed Method & $\begin{array}{l}\text { Slope of the } \\
\text { Linear Regression }\end{array}$ & $\begin{array}{l}\text { Intercept of the } \\
\text { Linear Regression }\end{array}$ \\
\hline Mackey-Galue \\
Mackey-Glass - FFNN & 1.00 & 0.00170 & 0.99866 \\
Logistic - FFNN & 1.00 & 0.00068 & 0.99960 \\
Logistic - Elman & 1.00 & 0.00019 & 0.99993 \\
Henon - FFNN & 1.00 & 0.00011 & 0.99999 \\
Henon - Elman & 0.99 & 0.00280 & 0.99928 \\
\hline
\end{tabular}




\section{REFERENCES}

[1] Zhang, J., Man, K.F. (1998) Time series prediction using recurrent neural network in multi-dimension embedding phase space. IEEE International Conference on Systems, Man, and Cybernetics. Vol. 2, pp. 11-14.

[2] Aref, H. (1984) Stirring by chaotic advection. Journal of Fluid Mechanics. Vol. 143, pp.1-21.

[3] Aubry, N., Holmes, P.J., Lumley, J.L., Stone, E. (1988) The dynamics of coherent structures in the wall region of a turbulent boundary layer. Journal of Fluid Mechanics. Vol. 192, pp.115-173.

[4] MacKay, R.S. (1993) Some thoughts on chaos in engineering. $7^{\text {th }}$ Toyota Conference: Toward the Harnessing of Chaos. Shizuoka, Japan.

[5] Erramilli, A., Forys, L.J. (1991) Oscillations and chaos in a flow model of a switching system. IEEE Journal of information systems: Logistics Information Management. Vol. 6 (4), pp.15-25.

[6] Uzunoglu, V., White, M.H. (1985) The synchronous oscillator: a synchronization and tracking network. IEEE Journal of Solid State Circuits. Vol. 20(6), pp. 1214-1225.

[7] Scott, S. (1991) Chemical Chaos. Oxford University Press.

[8] Weiss, G. (1992) Chaos hits Wall Street - the theory that is. Business Week. November 2, pp. 138-140.

[9] Qian, B., Rasheed, K. (2007) Stock market prediction with multiple classifiers. Applied Intelligence. Vol. 26, pp. 25-33.

[10] Das, A., Das, P. (2007) Chaotic analysis of the foreign exchange rates. Applied Mathematics and Computation. Vol. 185, pp. 388-396.

[11] Kennel, M.B. (1992) Method to distinguish possible chaos from colored noise and to determine embedding parameters. Physical Review A. Vol. 46, pp. 3111-3118.

[12] Wilding, R. (1998) Chaos, complexity and supply-chains. Logistics Focus. Vol. 6, pp. 8-10.

[13] Nair, A.S., Liu, J.C., Rilett, L., Gupta, S. (2001) Non-linear analysis of traffic flow. In Proceedings of IEEE Intelligent Transportation Systems Conference. pp. 25-29.

[14] Kawauchi, S., Sugihara, H., Sasaki, H. (2004) Development of very-short-term load forecasting based on chaos theory. Electrical Engineering in Japan. Vol. 148(2), pp. 55-63. 
[15] Lorenz, E.N. (1993) The Essence of Chaos. Seattle, University of Washington Press.

[16] Park, Y.R., Murray, T.J., Chen, C. (1996) Predicting sun spots using a layered perceptron neural network. IEEE Transactions on Neural Networks. Vol. 1(2), pp. $501-505$.

[17] González, J.J., Pereda, E. (2004) Applications of fractal and non-linear time series analysis to the study of short-term cardiovascular control. Current Vascular Pharmacology., Vol. 2, pp. 149-162.

[18] Casdagli, M. (1991) Chaos and deterministic versus stochastic non-linear modelling. in: Journal Royal Statistics Society: Series B, Vol. 54(2), pp.303-328.

[19] Wilding, R. (1998) Chaos theory: implications for supply chain management. International Journal of Logistics Management. Vol. (9), pp. 43-56.

[20] Takens, F. (1981) Detecting strange attractors in turbulence. in Dynamic Systems and Turbulence, Lecture Notes in Mathematics. Vol. (898), pp.366-381.

[21] Frazier, C., Kockelman, K. M. (2004) Chaos theory \& transportation systems: an instructive example. Transportation Research Record. Vol. (1897), pp. 9-17.

[22] Martinez-Rego, D., Fontenla-Romero, O., Alonso-Betanzos, A. (2008) A method for time series prediction using a combination of linear models. In Proceedings of European Symposium on Artificial Neural Network - Advances in Computational Intelligence and Learning, Bruges, Belgium.

[23] Casdagli M. (1989) Nonlinear prediction of chaotic time series. Physica D. Vol. 35, pp. 335-356.

[24] Farmer, J.D., Sidorowic, J.J. (1987) Predicting chaotic time series. Physical Review Letters. Vol. 8(59), pp. 845-848.

[25] McNames, J., (1998) A nearest trajectory strategy for time series prediction. In Proceedings of International Workshop on Advanced Black-Box Techniques for Nonlinear Modelling. Katholieke Universiteit Leuven, Belgium, pp. 112-128.

[26] Liu, F., Quek, C., Ng, G.S. (2005) Neural network model for time series prediction by reinforcement learning. In Proceedings of International Joint Conference on Neural Networks. Vol. 2, pp. 809-814. 
[27] Ma, Q., Zheng, Q., Peng, H., Zhong, T., Xu, L. (2007) Chaotic time series prediction based on evolving recurrent neural networks. In Proceedings of Sixth International Conference on Machine Learning and Cybernetics. Hong Kong.

[28] Menezes, J.M., and Barreto, G.A. (2008) Long-term time series prediction with the NARX network: an empirical evaluation. Neurocomputing. Vol. 71, pp. 3335-3343.

[29] Diaconescu, E., (2008) The use of NARX neural networks to predict chaotic time series. WSEAS Transactions on Computer Research. Vol. 3 (3), pp. 182-191.

[30] Tao, D., Xiao, H. (2007) Chaotic time series prediction based on radial basis function network. In Proceedings of 8th ACIS International Conference on Software Engineering, Artificial Intelligence, Networking, and Parallel/Distributed Computing. Vol. 1, pp. 595-599.

[31] Rojas, I., Valenzuelab, O., Rojasa, F., Guillena, A., Herreraa, L.J., Pomaresa, H., Marquezb, L., Pasadasb, M. (2008) Soft-computing techniques and ARMA model for time series prediction. Neurocomputing. Vol. 71, pp. 519-537.

[32] Koskela, T., Varsta, M. Heikkonen, J. Kaski, S. (1998) Time series prediction using recurrent SOM with local linear models. International Journal of Knowledge-based Intelligent Engineering Systems. Vol. 2(1), pp. 60-68.

[33] Barreto, G.A. (2006) Time series prediction with the self-organizing map: A review. Perspectives on Neural-Symbolic Integration. Vol. 77, pp. 135-158.

[34] Sapankevych, N., Sankar, R. (2009) Time series prediction using support vector machines: A survey. IEEE Computational Intelligence Magazine. Vol. 4 (2), pp. 24-38.

[35] Wang, X., Zhang, H., Zhang, C., Cai, X., Wang, J. (2005) Prediction of chaotic time series using LS-SVM with automatic parameter selection. In Proceedings of Sixth International Conference on Parallel and Distributed Computing - Applications and Technologies. pp. 962-965.

[36] Szpiro, G. (1997) Forecasting chaotic time series with genetic algorithms. Physical Review E. Vol. 55(3), pp. 2557-2568.

[37] Meyer, T., Packard, N.H. (1992) in Nonlinear Modeling and Forecasting, edited by M. Casdagli and S. Eubanks, SFI Studies in the Sciences of Complexity, AddisonWesley, New York, pp. 249-264. 
[38] Kodogiannis, V., Lolis, A. (2002) Forecasting financial time series using neural network and fuzzy system-based techniques. Neural Computing and Applications. Vo.11, pp. 90-102.

[39] Zhang, J., Chung, H. S., Lo, W. (2008) Chaotic time series prediction using a neurofuzzy system with time-delay coordinates. IEEE Transactions on Knowledge and Data Engineering. Vol. 20(7), pp. 956-964.

[40] Garcia-Trevino, E.S., Alarcon-Aquino, V. (2006) Single-step prediction of chaotic time series using wavelet-networks. In Proceedings of Electronics, Robotics and Automotive Mechanics Conference. Vol. 1, pp. 243-248.

[41] Wang, Y., Jiang, W., Yuan, S., Wang, J. (2008) Forecasting chaotic time series based on improved genetic Wnn. In Proceedings of Fourth International Conference on Natural Computation. Jinan, China.

[42] Wichard, J.D., Ogorzalek M. (2004) Time series prediction with ensemble models. In Proceedings of the IJCNN. Vol. 2, pp.1625-1629.

[43] Kim, Y.S., Yum, B.J. (2004) Robust design of multilayer feedforward neural networks: an experimental approach. Engineering Applications of Artificial Intelligence. Vol. 17(3), pp. 249-263.

[44] Khaw, J.F.C., Lim, B.S., Lim, L.E.N. (1995) Optimal design of neural networks using Taguchi method. Neurocomputing. Vol. 7(3), pp. 225-245.

[45] Lin, T.Y., Tseng, C.H. (2000) Optimum design for artificial neural networks: an example in a bicycle derailleur system. Engineering Applications of Artificial Intelligence. Vol. 13(1), pp. 3-14.

[46] Ortiz-Rodríguez, J.M., Martínez-Blanco, M.R., Vega-Carrillo, H.R. (2006) Robust design of artificial neural networks applying the Taguchi methodology and DOE. Proceedings of Electronics, Robotics and Automotive Mechanics Conference. Vol. 3, pp. 131-136.

[47] Packianather, M.S., Drake, P.R. (2004) Modelling neural network performance through response surface methodology for classifying wood veneer defects. Proceedings of the Institution of Mechanical Engineers, Part B: Journal of Engineering Manufacture. Vol. 218(4), pp. 459-466. 
[48] Wang, Q., Stockton, D.J., Baguley, P. (2000) Process cost modeling using neural networks. International Journal of Production Research. Vol. 38(16), pp. 3811-3821.

[49] Yang, S.M., Lee, G.S. (1999) Neural network design by using Taguchi method. Journal of Dynamic Systems, Measurement, and Control. Vol. 121, pp. 560-563.

[50] Peterson, G.E., St. Clair, D.C., Aylward, S., Bond, W. (1995) Using Taguchi's method of experimental design to control errors in layered perceptrons. IEEE Transactions on Neural Networks. Vol. 6(4), pp. 949-961.

[51] Mouli, K.C., Srinivas, J., Subbaiah, K.V. (2006) Optimisation and output forecasting using Taguchi-neural network approach. J. of the Institution of Engineers. pp. 54-57.

[52] Newton, I. (1729) The Mathematical Principles of Natural Philosophy. trans. A. Motte. Benjamin Motte, London.

[53] Wilding, R.D. (1997) An Investigation into Sources of uncertainty within Industrial Supply Chains; Amplification, Deterministic Chaos \& Parallel Interactions. PhD Dissertation, University of Warwich.

[54] Stewart, I. (1989) Does God Play Dice? Penguin, London.

[55] Crutchfield, J.P., Farmer, J.D., Packard, N.H. and Shaw, R.S. (1986) Chaos. Scientific America. Vol. 254 (12), pp. 46-57.

[56] Campbell, L., Garnett, W. (1882) The Life of James Clerk Maxwell. MacMillan and Co., London.

[57] Cartwright, M.L. (1965) Jacques Hadamard, 1865-1963. The Royal Society.

[58] Peitgen, H.O., Jurgens, H., Saupe, D. (1992) Chaos and Fractals: New Frontiers of Science, Springer-Velag, New York.

[59] Li, T.Y., Yorke J.A. (1975) Period three implies chaos. American Mathematical Monthly. Vol. 82(10), pp. 985-992

[60] Stacey, R.D. (1993) Strategic Management and Organizational Dynamics. Pitman Publishing, London.

[61] Kaplan, D., Glass, L. (1995) Understanding Nonlinear Dynamics, Springer-Verlag, NY.

[62] Abarbanel, H. (1996) Analysis of Observed Chaotic Data. Spinger-Verlag, New York.

[63] Box, G.E.P., Jenkins, G.M. (1976) Time Series Analysis, Forecasting and Control. Holden-Day, Oakland, CA, Revised version. 
[64] Pollok, D.S.G. (1999) A Handbook of Time Series, Signal Processing and Dynamics. Academic Press, London.

[65] Regonda, S.K., Rajagopalan, B., Lall, U., Clark, M., Moon, Y.I. (2005) Local polynomial method for ensemble forecast of time series. Nonlinear Processes in Geophysics. Vol. 12, pp. 397-406.

[66] Lau, K.W., Wu, Q.H. (2008) Local prediction of non-linear time series using support vector regression. Pattern Recognition. Vol. 41, pp. 1556-1564.

[67] Khashei, M., Bijari, M., Raissi Ardali, G.A. (2009) Improvement of auto-Regressive integrated moving average models using fuzzy logic and artificial neural networks (ANNs). Neurocomputing. Vol. 72, pp. 956-967.

[68] Thissen, U., Brakel, R., Weijer, A.P., Melssen, W.J., Buydens, L.M.C. (2003) Using support vector machines for time series prediction. Chemometrics and Intelligent Laboratory Systems. Vol. 69, pp. 35- 49.

[69] Zhang, W., Wu, Z., Yang, G. (2004) Genetic programming-based chaotic time series modeling. Journal of Zhejiang University Science. Vol. 5(11), pp. 1432-1439.

[70] Edmonds, A.N. (1996) Time series prediction using supervised learning and tools from chaos theory. PhD Dissertation, University of Luton.

[71] Jayawardena, A.W., Lai, F. (1993) Chaos in hydrological time series. Proceedings of the Yokohama Symposium - Extreme Hydrological Events: Precipitation, Floods and Droughts. pp.59-66.

[72] Sannasiraj, S.A., Zhang, H., Babovic, V. Chan, E.S. (2004) Enhancing tidal prediction accuracy in a deterministic model using chaos theory. Advances in Water Resources. Vol. 27, pp. 761-772.

[73] Sheikhan, M., Movaghar B. (2009) Exchange rate prediction using an evolutionary connectionist model. World Applied Sciences Journal. Vol. 7, pp. 8-16.

[74] Gleick, J. (1987) Chaos - Making a new science. Viking, New York.

[75] Boné, R., Crucianu, M. (2002) Multi-step-ahead prediction with neural networks: a review. In 9emes rencontres internationales: Approches Connexionnistes en Sciences. Boulogne, France. pp. 97-106. 
[76] Bontempi, G, Birattari, M., Bersini, H. (1999) Local learning for iterated time-series prediction. Proceedings of the Sixteenth International Conference on Machine Learning. San Francisco, USA, pp. 32-38.

[77] Lorenz, E.N. (1969) Atmospheric predictability as revealed by naturally occurring analogues. Journal of the Atmospheric Sciences. Vol. 26, pp. 636-646.

[78] Jacobs, R.A., Jordan, M.I., Nowlan, S.J., Hinton, G.E. (1991) Adaptative mixtures of local experts. Neural Computation. Vol. 3, pp. 79-87.

[79] Zhang, J., Man K. F., Ke J.Y. (1998) Time series prediction using Lyapunov exponents in embedding phase space. Proceedings Fourth International Conference on Signal Processing. Vol. 1, pp. 221-224.

[80] Chudy, L., Farkas, I. (1998) Prediction of chaotic time-series using dynamic cell structures and local linear models. Neural Network World. Vol. 8(5), pp. 481-489.

[81] Kugiumtzis, D., Lingjaerde, O.C., Christophersen, N. (1998) Regularized local linear prediction of chaotic time series. Physica D. Vol. 112, pp. 344-360.

[82] Sello, S. (2001) Solar cycle forecasting: a nonlinear dynamics approach. Astronomy \& Astrophysics. Vol. 377, pp. 312-320.

[83] Fontenla-Romero, O., Alonso-Betanzos, A., Castillo, E., Principe, J.C., GuijarroBerdinas, B. (2002) Local modeling using self-organizing maps and single layer neural networks. In Lecture Notes in Computer Science. Vol. 2415, pp. 945-950.

[84] Gholipour, A., Araabi, B.N., Lucas, C. (2006) Predicting chaotic time series using neural and neurofuzzy models: a comparative study. Neural Processing Letters, Vol. 24, pp. 217-239.

[85] Granger, C.W.J., Anderson A.P (1978) An Introduction to Bilinear Time Series Models, Vandenhoeck and Ruprecht, Gottingen.

[86] Subba-Rao, T., Gabr M.M. (1984) An introduction to bispectral analysis and bilinear time series models. Lecture Notes in Statistics. Vol. 24, pp. 270-275.

[87] Ozaki, T., Oda, H. (1978) Nonlinear time series model identification by Akaike's information criterion. Information and Systems. pp. 83-91.

[88] Haggan, V. and T. Ozaki (1981) Modeling nonlinear random vibrations using an amplitudedependent autoregressive time series model, Biometrika. Vol. 68, pp. 189-196. 
[89] Priestley, M.B. (1980) State dependent models: a general approach to nonlinear time series analysis. Journal of Time Series Analysis. Vol. 1, pp. 57-71.

[90] Priestley, M.B. (1988) Non-linear and Non-stationary Time Series Analysis. Academic Press, London.

[91] Tong, H., Lin, K.S. (1980) Threshold auto-regression, limit cycles and cyclical data. Journal of the Royal Statistical Society. Vol. 42(3), pp. 245-292.

[92] Tong, H. (1983) Threshold Models in Nonlinear time Series Analysis. Springer Verlag, New York.

[93] Martinez, T.M., Berkovich, S.G., Schulten, K.J. (1993) Neural-gas network for vector quantization and its application to time-series prediction. IEEE Transactions on Neural Networks. Vol. 4, pp. 558-569.

[94] Bersini, H., Birattari, M., Bontempi, G. (1998) Adaptive memory-based regression methods. In Proceedings of the IEEE International Joint Conference on Neural Networks. pp. 2102-2106.

[95] Girard, A., Rasmussen, C.E., Qui, J. (2003) Gaussian process priors with uncertain inputs-Application to multiple-step ahead time series forecasting. In Advances in Neural Information Processing Systems. Vol. 15, pp. 529-536.

[96] Jaeger, H., Haas, H. (2004) Harnessing nonlinearity: Predicting chaotic systems and saving energy in wireless communication. Science. Vol. 304 (5667), pp. 78-80.

[97] Chen, Y., Yang, B., Dong, J., Abraham, A. (2005), Time-series forecasting using flexible neural tree model. Information Sciences. Vol. 174, pp. 219-235.

[98] Chen, Y.M, Lin, C. (2007) Dynamic parameter optimization of evolutionary computation for on-line prediction of time series with changing dynamics. Applied Soft Computing. Vol. 7, pp. 1170-1176.

[99] Elsner, J.B., (1992) Predicting time series using a neural network as a method of distinguishing chaos from noise, Journal of Physics A: Mathematical and General. 25 (4), pp. 843-850.

[100] Veelen, M., Nijhuis, J., Spaanenburg, B. (2000) Neural network approaches to capture temporal information. In Computing Anticipatory Systems - Third International Conference. Vol. 517, pp. 361-371. 
[101] Moller, M. (1993) A scaled conjugate gradient algorithm for fast supervised learning. Neural Networks. Vol. 6, pp. 525-533.

[102] Tenti, P. (1996) Forecasting foreign exchange rates using recurrent neural networks. Applied Artificial Intelligence. Vol. 10, pp. 567-581.

[103] Connor, J., Atlas, L. (1991) Recurrent neural networks and time series prediction. IEEE International Joint conference on Neural Networks. pp. 301-306.

[104] Connor, J.T., Martin D. (1994) Recurrent neural networks and robust time series prediction. IEEE Transactions on Neural Networks. Vol. 5(2), pp. 240 - 254.

[105] Bone, R., Crucianu, M., Verley, G., Asselin de Beauville, J.P. (2000) A bounded exploration approach to constructive algorithms for recurrent neural networks. In Proceedings of IJCNN. Como, Italy, Vol. 3, pp. 27-30.

[106] Han, M., Xi, J., Xu, S., Yin, F. (2004) Prediction of chaotic time series based on the recurrent predictor neural network. IEEE Transactions on Signal Processing. Vol. 52(12), pp. 3409-3416.

[107] Assaad, M., Boné, R., Cardot, H. (2006) Predicting chaotic time series by boosted recurrent neural networks. In Proceedings of International Conference on Neural Information Processing. Vol. 4233, pp. 831-840.

[108] Guan, X., Mural, R.J., Uberbacher, E.C. (1994) Protein structure prediction using hybrid AI methods. In Proceedings of the Tenth Conference on Artificial Intelligence for Applications. pp. 471-473.

[109] Lin, J., Bartal, Y., Uhrig, R. (1995) Using similarity based formulas and genetic algorithms to predict the severity of nuclear power plant transients. $9^{\text {th }}$ Power Plant Dynamics, Control \& Testing Symposium. Knoxville, TN, pp. 53.01-53.09.

[110] Leung, H. (2001) Prediction of noisy chaotic time series using an optimal radial basis function neural network. IEEE Transactions on Neural Networks. Vol. 12(5), pp. 1163-1172.

[111] Haykin S., Principe, J. (1998) Making sense of a complex world. IEEE Signal Processing Magazine. Vol. 15(3), pp. 66-68. 
[112] Cowper, M.R., Mulgrew, B., Unsworth, C.P. (2002) Nonlinear prediction of chaotic signals using a normalized radial basis function network. Signal Processing. Vol. 82(5), pp. 775-789.

[113] Poggio, T., Girosi, F. (1989) A theory of networks for approximation and learning. Massachusetts Institute of Technology, Cambridge, MA.

[114] Girosi, F., Jones, M., Poggio, T. (1995) Regularization theory and neural network architectures. Neural Computation. Vol. 7(2), pp. 219-269.

[115] Cho, K.B., Wang, H.B. (1996) Radial basis function based adaptive fuzzy systems and their applications to system identification and prediction. Fuzzy Sets and Systems. Vol. 83(3), pp. 325-339.

[116] Rojas, I., Pomares, H., Bernier, J.L., Ortega, J., Pino, B., Pelayo, F.J., Prieto, A. (2002) Time series analysis using normalized PG-RBF network with regression weights, Neurocomputing. Vol. 42, pp. 267-285.

[117] Vesin, J.M. (1993) An amplitude-dependent autoregressive signal model based on a radial basis function expansion. IEEE International Conference on Acoustics, Speech, and Signal Processing. Vo. 3, pp. 129-132.

[118] Shi, Z., Y. Tamura, and T. Ozaki (1999) Nonlinear time series modelling with the radial basis function-based state-dependent autoregressive model. International Journal of Systems Science. Vol. 30(7), pp. 717-727.

[119] Peng, H., Ozaki, T., Toyoda, Y., Oda, K. (2001) Nonlinear system identification using radial basis function-based signal-dependent ARX models. Proceedings of 5th IFAC Symposium on Nonlinear Control Systems. Saint-Petersburg, Russia, pp. 703-708.

[120] Peng, H., Ozaki, T., Haggan-Ozaki, V. and Toyoda, Y. (2003) A parameter optimization method for radial basis function type models. IEEE Transactions on Neural Networks. Vol. 14, pp. 432-438.

[121] Haggan-Ozaki, V., Ozaki, T. Toyoda, Y. (2006) RBF-ARX modeling for prediction and control. 14th IFAC Symposium on System Identification. Newcastle, Australia, pp. 1210-1215. 
[122] Teo, K., Wang, L., Lin, Z. (2001) Wavelet packet multi-layer perceptron for chaotic time series prediction: effects of weight initialization. Computational Science. Vol. 2074, pp. 310-317.

[123]Cao, L., Hong, Y., Fang, H. and He G. (1995) Predicting chaotic time series with wavelet networks. Physica D: Nonlinear Phenomena. Vol. 85 (1-2), pp. 225-238.

[124] Gao, X., Xiao, F., Zhang, J., Cao, C. (2004) Short-term prediction of chaotic time series by wavelet networks. Proceedings of the 5th World Congress on Intelligent Control and Automation. Hangzhou, China, Vol. 3, pp. 1931-1935.

[125] Gao,X., Xiao, F. (2004) Multiwavelet networks for prediction of chaotic time series. IEEE International Conference on Systems, Man and Cybernetics. Vol. 4, pp. 3328-3332.

[126] Wan, E.A. (1994) Time series prediction by using a connectionist network with internal time delays. in Time Series Prediction: Forecasting the Future and Understanding the Past. Addison-Wesley, pp. 195-217.

[127] Koskela, T., Lehtokangas, M. Saarinen, J., Kaski, K. (1996) Time series prediction with multilayer perceptron, FIR and Elman neural networks. In Proceedings of World Congress on Neural Networks. pp. 491-496.

[128] Day, S.P., Davenport, M.R. (1993) Continuous-time temporal backprogagation with adaptive time delays. IEEE Transactions on Neural Networks. Vol. 4, pp. 348-354.

[129] Yao, X., Liu, Y. (1997) A new evolutionary system for evolving artificial neural networks. IEEE Transactions on Neural Networks. Vol. 8(3), pp. 694-713.

[130] Hecht-Nielsen, R. (1988) Applications of countepropagation networks. Neural Networks. Vol. 1, pp. 131-139.

[131] Fierascu, C. (2005) Counterpropagation with Delays with applications in time series prediction. Artificial Neural Networks: Formal Models and Their Applications. Vol. 3697, pp. 533-539.

[132] Gomez-Ramırez , E., Najim, K., Ikonen, E. (2007), Forecasting time series with a new architecture for polynomial artificial neural network. Applied Soft Computing. Vol. 7, pp. 1209-1216.

[133] Smola, A.J., Scholkopf, B. (1998) A tutorial on support vector regression. Technical report. Statistics and Computing. Vol. 14 (3), pp. 199 - 222. 
[134] Muller, K.R., Smola, A.J., Ratsch, G., Scholkopf, B., Kohlmorgen, J., Vapnik, V. N. (1997) Predicting time series with support vector machines. In Proceedings of 7th International Conference of Artificial Neural Networks. Lausanne, Switzerland, Vol. 1327, pp. 999-1004.

[135] Mukherjee, S., Osuna, E. and Girosi, F. (1997) Nonlinear prediction of chaotic time series using support vector machines. In Proceedings of 7th IEEE Workshop Neural Networks Signal Processing. Amelia Island, FL, pp. 511-520.

[136] Cao, L.J., Tay, F.E.H. (2003) Support vector machine with adaptive parameters in financial time series forecasting. IEEE Transactions on Neural Networks. Vol. 14(6), pp. 1506-1518.

[137] Zheng, D., Wang, J., Zhao, Y. (2006) Time series predictions using multi-scale Support Vector Regressions. Theory and Applications of Models of Computation. Vol. 3959, pp. 474-481.

[138] Simon, G., Lendasse, A., Cottrell, M., Fort, J.C., Verleysen, M. (2005) Time series forecasting: obtaining long term trends with self-organizing maps. Pattern Recognition Letters. Vol. 26(12), pp. 1795-1808.

[139] Afolabi, M.O., Olude, O. (2007) Predicting stock prices using a hybrid Kohonen self organizing map (SOM). Proceedings of the 40th Hawaii International Conference on System Sciences. pp. 48.

[140] Zorin, A. (2003) Stock price prediction: Kohonen versus backpropagation. In Proceedings of the International Conference on Modeling and Simulation of Business Systems. Vilnius, Lithuania, pp. 115-119.

[141] Vesanto, J. (1997) Using the SOM and local models in time-series prediction. In Proceedings of Workshop on Self-Organizing Maps. Espoo, Finland, pp. 209-214.

[142] Lee, K.L. and Billings, S.A. (2002) Time series prediction using support vector machines, the orthogonal and the regularized orthogonal least-squares algorithms. International Journal of Systems Science. Vol. 33(10), pp. 811-821.

[143] Shi, Z. and Han, M. (2007) Support vector echo-state machine for chaotic, time series prediction. IEEE Transactions on Neural Networks. Vol. 18(2), pp. 359-372. 
[144] Palit, A.K., Popovic, D. (1999) Forecasting chaotic time series using neuro-fuzzy approach. International Joint Conference on Neural Networks. Washington D.C., USA, Vol.3, pp. 1538-1543.

[145] Jang, J.S.R., Sun, C.T. and Mizutani, E. (1997) Neuro-Fuzzy and Soft Computing. Englewood cliffs, New Jersey, Prentice-Hall.

[146] Falco, I., Iazzetta, A., Natale, P., Tarantino, E. (1998) Evolutionary neural networks for nonlinear dynamics modelling. In Parallel Problem Solving from Nature. pp. 593-602.

[147] Yoshihara, I., Ohta, K., Yamamori, K. (2003) Construction of GMDH-based prediction model using GA. Mem Fac Eng Miyazaki Univ. Vol. 32, pp.315-320.

[148] Luo, X. Heywood, M. and Zincir-Heywood, A.N. (2006) Benchmarking a recurrent linear GP model on prediction and control problems, intelligent control and automation. International Conference on Intelligent Computing. Kunming, China, Vol. 344, pp. 845-850.

[149] Day, P., Nandi, A.K. (2008) Sunspot prediction using genetic programming augmented by binary string fitness characterisation and comparative partner selection. IEEE Workshop on Machine Learning for Signal Processing. pp. 175-180.

[150] Jia-shu, Z., Xian-Ci, X. (2000) Predicting low-dimensional chaotic time series using volterra adaptive filer. Acta Physica Sinica. Vol. 49(3), pp. 403-408.

[151] Jia-shu, Z., Xian-Ci, (2000) Prediction of chaotic time series by using adaptive higher-order nonlinear Fourier infrared filter. Acta Physica Sinica. Vol. 49(7), pp. 1221-1227.

[152] Jia-shu, Z., Xian-Ci, (2000) Nonlinear adaptive prediction of chaotic time series with a reduced parameter nonlinear adaptive filter. Acta Physica Sinica. Vol. 49(12), pp. 2333-2339.

[153] Li, C., Yu, J. (2008) Volterra-TLS method for chaotic time series prediction. International Conference on Communications, Circuits and Systems. pp. 48-51.

[154] Kim, D. and Kim, C. (1997) Forecasting time series with genetic-fuzzy predictor ensemble. IEEE Transactions on Fuzzy Systems. Vol. 5(4), pp. 523-535. 
[155] Bakker, R., Schouten, J.C., Giles, C.L., Takens, F., Bleek, C.M. (2000) Learning chaotic attractors by neural networks. Neural Computation. Vol. 12(10), pp. 2355-2383.

[156] Fanzi, Z. Zhengding, Q. (2003) Predicting chaotic time series by ensemble self generating neural networks merged with genetic algorithm. Proceedings of the 2003 International Conference on Neural Networks and Signal Processing. Nanjing, China, Vol. 1, pp. 776-778.

[157] Bouchachia, A., Bouchachia, S. (2008) Ensemble learning for time series prediction. The First International Workshop on Nonlinear Dynamic Systems and Synchronization. pp. 1-8.

[158] Forrester, J.W. (1961) Industrial Dynamics, Systems Dynamics Series. Productivity Press (MIT Press), Portland, Oregon.

[159] Thompson, J.M.T., Stewart, H.B. (1986) Nonlinear Dynamics and Chaos. John Wiley \& Sons, UK.

[160] Gordon, T., Greenspan, D. (1994) The Management of chaotic systems. Technological Forecasting and Social Change. Vol. 47, pp. 49-62.

[161] Wolf, A., Swift, J.B., Swinney, H.L., Vastano, J.A. (1985) Determining Lyapunov exponents from a time series. Physica D. Vol. 16(3), pp. 285-317.

[162] Banerjee, S. (1993) Choas. Students Journal of the Institute of Electronics and Telecommunications Engineers. Vol. 34, pp. 39-50.

[163] Parker, D., Stacey, R. (1994) Chaos management and economics - the Implications of Non-linear Thinking. Hobart Paper 125, Institute of Economic Affairs, London.

[164] Goldratt, E.M., Cox, J. (1984) The Goal. 2nd Ed., Gower Publishing, London.

[165] Vliet, A. (1994) Order from chaos. Management Today. November, pp. 62-67.

[166] Norgaard, M., Ravn, O., Poulsen, N.K., Hansen, L.K. (2000) Neural Networks for Modelling and Control of Dynamic Systems. Springer, Berlin.

[167] Kantz, H., Scheiber, T. (1997) Nonlinear Time Series Analysis. Cambridge University Press, Cambridge.

[168] Camilleri, M. (2004) Forecasting using non-linear techniques in time series analysis: an overview of techniques and main issues. In Proceeding of Computer Science Annual Research Workshop. pp. 19-28. 
[169] Bloomfield, P. (2000) Fourier Analysis of Time Series: An Introduction. John Wiley \& Sons, New York.

[170] Addison, P. S. (1997) Fractals and Chaos: an Illustrated Course. Institute of Physics Publishing, Bristol.

[171] Sprott, J.C. (2003) Chaos and Time Series Analysis. Oxford University Press, UK.

[172] Hurst, H.E. (1951) Long term storage capacity of reservoirs. Transactions of the American Society of Civil Engineers. Vol. 116, pp. 770-799.

[173] Harris, C.M., Todd, R.W., Bungard, S.J., Lovitt, R.W., Morris, J.G., Kell, D.B. (1987) The dielectric permittivity of microbial suspensions at radio-frequencies: a novel method for the estimation of microbial biomass. Enzyme Microbial Technology. Vol. 9 (3), pp.181-186.

[174] Edman, A.N. (1996) Time Series Prediction Using Supervised Learning and Tools from Chaos Theory. PhD Thesis, University of Luton.

[175] Hilborn, R.C. (2001) Chaos and Nonlinear Dynamics: An Introduction for Scientists and Engineers. 2nd Edition, Oxford University Press, UK.

[176] Cao, L. (1997) Practical method for determining the minimum embedding dimension of a scalar time series. Physica D. Vol. 110, pp. 43-50.

[177] Luk, K.C., Ball, J.E. and Sharma, A. (2000) A study of optimal model lag and spatial inputs to artificial neural networks for rainfall forecasting. Journal of Hydrology. Vol. 227 (1-4), pp.56-65.

[178] Hagan, M.T., Demuth, H.B., Beale, M.H. (1996) Neural Network Design. PWS Publishing, Boston.

[179] Yilmaz, I., Yuksek, A.G. (2008) An example of artificial neural network application for indirect estimation of rock parameters. Rock Mechanics and Rock Engineering. Vol. 41(5), pp. 781-795.

[180] Koivo, H., Elmusrati, M. (2009) System Engineering in Wireless Communications. John Wiley \& Sons.

[181] Elman, J.L. (1990) Finding structure time. Cognitive Science, Vol. 14, pp. 179-211. 
[182] Leontaritis, I.J., Billings, S.A. (1985) Input-output parametric models for nonlinear systems - part I: deterministic nonlinear systems, International Journal of Control, Vol. 41(2), pp. 303-328.

[183] Ljung, L. (1999) System Identification: Theory for the User. 2nd Edition, PrenticeHall, Englewood Cliffs, New Jersey.

[184] Darwin, C.R. (1859) On the origin of species by means of natural selection, or the preservation of favoured races in the struggle for life. John Murray, London.

[185] Holland, J. (1975) Adaptation in Natural and Artificial Systems, University of Michigan Press, Ann Arbor, MI.

[186] Ardalani-Farsa, M., Zolfaghari, S. (2008) Chaotic time series prediction using chaos theory and artificial neural networks. Canadian Operational Research Society (CORS) conference. May 12-14, 2008, Quebec City, Canada (Abstract).

[187] Ardalani-Farsa, M., Zolfaghari, S. (2008) Synergy of chaos theory and artificial neural networks in time series prediction. In Manufacturing Fundamentals: Necessity and Sufficiency, Proceedings of the 3rd World Conference on Production and Operations Management. August 5-8, 2008, Tokyo, Japan. Chapter 20, pp. 2771-2716.

[188] Ardalani-Farsa, M., Zolfaghari,S. (2010) Synergy of chaos theory and artificial neural networks in chaotic time series forecasting. International Journal of Applied Management Science. Accepted July 22, 2010.

[189] MacKey, M.C., Glass, L. (1977) Oscillation and chaos in physiological control systems. Science. Vol. 197 (4300), pp. 287-289.

[190] Parlitz, U., I. Wedekind, W. Lauterborn, C. Merkwirth (2001) TSTOOL and User Manual, Ver. 1.11. http://www.physik3.gwdg.de/tstool.

[191] MATLAB Release 2009a, The MathWorks, Inc., 2009.

[192] Eckmann, J.P., Ruelle, D. (1985) Ergodic theory of chaos and strange attractors. Reviews of Modern Physics. Vol. 57(3), pp. 617-656.

[193] Moran P.A.P. (1950) Some remarks on animal populations. Biometrics. Vol. 6, pp. 250-258. 
[194] Ricker, B. (1954) Stock and recruitment. Journal of the Fisheries research Board of Canada. Vol. 11, pp. 559-663.

[195] May, L.R. (1976) Simple mathematical models with very complicated dynamics. Nature. Vol. 261, pp. 459-467.

[196] Zhang, J., Lamb, K.C., Yanc, W.J., Gaob, H., Lid, Y. (2004) Time series prediction using Lyapunov exponents in embedding phase space. Computers \& Electrical Engineering. Vol. 30(1), pp.1-15.

[197] Hénon, M. (1976) A two-dimensional mapping with a strange attractor. Communications in Mathematical Physics. Vol. 50, pp. 69-77.

[198] Davies, B. (1999) Exploring Chaos, theory and experiment. Perseus Books, Reading, Massachusetts.

[199] Ardalani-Farsa, M., Zolfaghari, S. (2009) Residual Analysis and combination of embedding theorem and artificial intelligence in chaotic time series prediction. The 29th International Symposium on Forecasting. June 21-24, 2009, Hong Kong.

[200] Ardalani-Farsa, M., Zolfaghari, S. Residual analysis and combination of embedding theorem and artificial intelligence in chaotic time series forecasting (under review).

[201] Ardalani-Farsa, M., Zolfaghari, S. (2010) Forecasting chaotic time series with residual analysis method using ensemble Elman-NARX neural networks. In Proceedings of the Industrial Engineering Research Conference (IERC 2010). June 5-9, 2010, Cancun, Mexico.

[202] Ardalani-Farsa, M., Zolfaghari, S. (2010) Chaotic time series prediction with residual analysis method using hybrid Elman-NARX neural networks. Neurocomputing. Vol. 73 (13-15), pp. 2549-2553.

[203] Lorenz, E.N. (1963) Deterministic non-periodic flows. Journal of Atmospheric Science. Vol. 20, pp. 130-141.

[204] SIDC (World Data Center for the Sunspot Index), http://sidc.oma.be/index.php3.

[205] Wang, L.X., Mendel, J.M. (1992) Generating fuzzy rules by learning from examples. IEEE Transactions on Systems, Man and Cybernetics. Vol. 22(6), pp.1414-1427. 
[206] Inoue, H., Fukunaga, Y., Narihisa, H. (2001) Efficient hybrid neural network for chaotic time series prediction. In Proceedings of International Conference on Artificial Neural Networks. pp.712-718.

[207] Iokibe, T., Fujimoto, Y., Kanke, M., Suzuki, S. (1997) Short-term prediction of chaotic time series by local fuzzy reconstruction method. Journal of Intelligent and Fuzzy System. Vol. 5, pp. 3-21.

[208] Dhahri, H., Alimi, A.M. (2006) The modified differential evolution and the RBF (MDE-RBF) neural network for time series prediction. In Proceedings of International Joint Conference on Neural Networks. pp. 2938-2943.

[209] McNish, A.G., Lincoln, J.V. (1949) Prediction of sunspot numbers. Transactions on the American Geophysical Union. Vol. 30(5), pp. 673-685.

[210] Denkmayr, K., Cugnon, P. (1997) About sunspot number medium-term predictions. In proceedings of the Solar-Terrestrial Prediction Workshop V. Solar Terrestrial Research Center, Japan, pp.103.

[211] Ardalani-Farsa, M., Zolfaghari, S. (2009) Synergy of embedding theorem and optimized neural network using Taguchi's design of experiments in time series forecasting. International Conference on Industrial Engineering and Systems Management (IESM 2009). May 13-15, 2009, Montreal, Canada.

[212] Ardalani-Farsa, M., Zolfaghari, S. Taguchi's design of experiment in combination selection for a chaotic time series forecasting method using ensemble artificial neural networks (under review).

[213] Box, G.E.P., Hunter, W.G., Hunter, S. (1987) Statistics for Experimenters: An Introduction to Design Data Analysis, and Model Building. Wiley, New York.

[214] Montgomery, D.C. (2001) Design and Analysis of Experiments. John Wiley \& Sons, New York.

[215] Chen, Y., Tam, S.C., Chen, W.L., Zheng, H.Y. (1996) Application of Taguchi method in the optimization of laser micro-engraving of photomasks. International Journal of Materials and Product Technology. Vol.11 (3-4), pp. 333-344.

[216] Jiju, A., Frenie, A.J. (2001) Teaching the Taguchi method to industrial engineers. MCB University press. Vol. 50 (4), pp. 141-149. 
[217] Taguchi, G. (1986) Introduction to Quality Engineering. Kraus International Publications, Asian Productivity Organization, White Plains, New York.

[218] Wang, S.M., Giang, Y.S., Ling, Y.C. (2002) Taguchi's method in optimizing the experimental conditions of simultaneous supercritical fluid extraction and chemical derivatization for the gas chromatographic-mass spectrometric determination of amphetamine and methamphetamine in aqueous matrix. Forensic Science Journal. Vol. 1 (1), pp. 47-53.

[219] Naidu, N.V.R., Gowda, D. (2001) Optimising process parameters using Taguchi techniques for quality improvement- a case study. Industrial Engineering Journal. Vol. 30, pp. 29-35.

[220] Gandhi, A. (2003) Problem solving using Taguchi DOE techniques. Industrial Engineering Journal. Vol. 32, pp. 16-25.

[221] Manna, A., Bhattacharyya, B. (2003) Taguchi method based parametric study of $\mathrm{CNC}$-wire cut $\mathrm{EDM}$ during machining of $\mathrm{Al} / \mathrm{SiC}$. Journal of the Institution of Engineers. Vol. 83 (2), pp. 62-69.

[222] Sukthomya, W., Tannock, J. (2005) The optimisation of neural network parameters using Taguchi's design of experiments approach: an application in manufacturing process modeling. Neural Computing \& Applications. Vol. 14, pp. 337-344.

[223] Mezgar, I., Egrosts, C., Monoston, L. (1997) Design and real time reconfiguration of robust manufacturing systems by using DOE and neural networks. Computers in Industry. Vol. 33, pp. 61-70.

[224] Tsai, C. S., Mort, N. (1996) Simulation and optimisation in manufacturing systems using Taguchi methods. Proceedings of UKACC International Conference on Control. Vol.1, pp. 467-472.

[225] Minitab 15® Statistical Software.

[226] Huebner, U., Abraham, N.B., Weiss, C.O. (1989) Dimensions and entropies of chaotic intensity pulsations in a single-mode far-infrared NH3 laser. Physical Review A. Vol. 40(11), pp. 6354-6365.

[227] Weigend, A., Gershenfeld, N., (1993) Time series prediction: forecasting the future and understanding the past. Addision-Wesley, Reading, MA. 
[228] Dorffner, G., Bischof, H., Hornik, K. (2001) Applying LSTM to time series predictable through time-window approaches. Lecture Notes in Computer Science. Vol. 2130, pp. 669-676.

[229] Weigend, A.S., Nix, D.A. (1994) Predictions with confidence intervals (local error bars). Proceedings of the International Conference on Neural Information Processing. Seoul, Korea. pp. 847-852.

[230] Baumol, W., Benhabib, J. (1989) Chaos: significance, mechanism, and economic applications. Journal of Economic Perspectives. Vol. 3, pp. 77-105.

[231] Kelsey, D. (1988) The economics of chaos or the chaos of economics. Oxford Economic Papers. Vol. 40, pp. 1-31.

[232] Kauffman, S.A. (1991) Antichaos and adaptation. Scientific American. Vol. 265(2), pp. 78-84.

[233] Mayer-Kress, G., Grossman, S. (1989) Chaos in the international arms race. Nature. Vol. 337, pp. 701-704.

[234] Goldberger, A.L., Rigney, D.R., West, B.J. (1990) Chaos and fractals in physiology. Scientific American. Vol. 263, pp. 43-49.

[235] Stapleton, D., Hanna, J.B., Ross, J.R. (2006) Enhancing supply chain solutions with the application of chaos theory. Supply Chain Management: An International Journal. Vol. 11(2), pp. 108-114.

[236] Levy, D. (1994) Chaos theory and strategy: theory, application and managerial implications. Strategic Management Journal. Vol. 15, pp. 167-178.

[237] Wichard, J.D., Parlitz, U., Lauterborn, W. (2000) Nonlinear time series analysis in production systems, attractors, signals, and synergetic. Proceedings of the 1st European Interdisciplinary School on Nonlinear Dynamics for System and Signal Analysis. pp. 261-267.

[238] Feichtinger, G. (1996) Chaos theory in operations research. International Transactions in Operational Research. Vol. 3(1), pp. 23-36.

[239] Hibbert B., Wilkinson, I.F. (1994) Chaos theory and the dynamics of marketing systems. Journal of the Academy of Marketing Science, Vol. 22(3), pp. 218-233. 
[240] Gori, F., Ludovisi, D., Cerritelli, P.F. (2007) Forecast of oil price and consumption in the short term under three scenarios: Parabolic, linear and chaotic behaviour. Energy. Vol. 32, pp. 1291-1296.

[241] Shang, P., Li, X., Kamae, S. (2005) Chaotic analysis of traffic time series. Chaos, Solutions and Fractals. Vol. 25, pp. 121-128.

[242] Jiang, C., Li, T. (2005) Forecasting method study on chaotic load series with high embedded dimension. Energy Conversion and Management. Vol. 46, pp. 667-676.

[243] Haase, P., Schlink, U., Richter, M. (2002) Critical reconsideration of phase space embedding and local non-parametric prediction of ozone time series. Water, Air, and Soil Pollution: Focus. Vol. 2, pp. 513-524.

[244] Kuo, R.J., Lee, L.C., Lee, C.F. (1996) Integration of artificial NN and fuzzy delphi for stock market forecasting. IEEE International Conference on Systems, Man, and Cybernetics. Vol. 2, pp. 1073-1078.

[245] Hassan M.R., Nath, B. (2005) Stock market forecasting using Hidden Markov Model: A new approach. Proceedings of the 5th International Conference on Intelligent Systems Design and Applications. pp. 192-196.

[246] Hsieh, D.A. (1991) Chaos and nonlinear dynamics: Application to financial markets. Journal of Finance. Vol. 46(5), pp. 1839-1877.

[247] Peters, E.E. (1991) Chaos and order in the capital markets: a new view of cycles, prices, and market volatility. John Wiley \& Sons, New York.

[248] Peters, E.E. (1994) Fractal market analysis: applying chaos theory to investment and economics. John Wiley \& Sons, New York.

[249] Abu-Mostafa, Y.S, Atiya, A.F. (1996) Introduction to financial forecasting. Applied Intelligence. Vol. 6, pp. 205-13.

[250] Huanga, W., Nakamoria, Y., Wangb, S. (2005) Forecasting stock market movement direction with support vector machine. Computers and Operations Research. Vol. 32 (10), pp. 2513-2522.

[251] Saad, E.W., Prokhorov, D.V., Wunsch, D.C. (1998) Comparative study of stock trend prediction using time delay, recurrent and probabilistic neural networks. IEEE Transactions on Neural Networks. Vol. 9 (6), pp. 1456-1470. 
[252] Cecen, A.A., Erkal, C. (1996) Distinguishing between stochastic and deterministic behaviour in foreign exchange rate returns: further evidence. Economics Letters. Vol. 51, pp. 323-329.

[253] Bask, M. (1996) Dimensions, and Lyapunov exponents from exchange rate series. Chaos, Solitons \& Fractals. Vol. 7(12), pp. 2199-2214.

[254] Bask, M. (2002) A positive Lyapunov exponent in Swedish exchange rates? Chaos, Solitons \& Fractals. Vol.14(5), pp. 1295-1304.

[255] Liu, W. (2008) Forecasting exchange rate change between USD and JPY by using dynamic adaptive neuron-fuzzy logic system. Asia Pacific Journal of Finance and Banking Research. Vol. 2(2), pp. 1-12.

[256] Taylor, S.J. (1994) Trading futures using a channel rule: A study of the predictive power of technical analysis with currency examples. Journal of Futures Markets. Vol. 14, pp. 215-235.

[257] Taylor, S.J. (1986) Modelling financial time series. John Wiley \& Sons, New York.

[258] Brock, W.A., Hsieh, D.A., LeBaron, B. (1991) Nonlinear dynamics, chaos, and instability. MIT Press, Cambridge, Massachusetts.

[259] De Grauwe, P., Dewachter, H., Embrechts, M. (1993) Exchange rate theory. Chaotic models of foreign exchange markets. Blackwell, London.

[260] Fang, H., Lai, K.S., Lai., M. (1994) Fractal structure in currency futures price dynamics. Journal of Futures Markets. Vol. 14, pp. 169-181.

[261] LeBaron, B. (1994) Chaos and nonlinear forecastability in economics and finance, Philosophical Transactions of the Royal Society A. Vol. 348, pp. 397-404.

[262] Richards, G.R. (2000) The fractal structure of exchange rates: measurement and forecasting. Journal of International Financial Markets. Vol. 10, pp. 163-180.

[263] Schwartz, B., Yousefi, S. (2003) On complex behavior and exchange rate dynamics. Chaos, Solitons and Fractals. Vol. 18, pp. 503-523.

[264] Guegan, D., Mercier, L. (2005) Prediction in chaotic time series: methods and comparisons with an application to financial intra-day data. The European Journal of Finance. Vol. 11(2), pp. 137-150. 